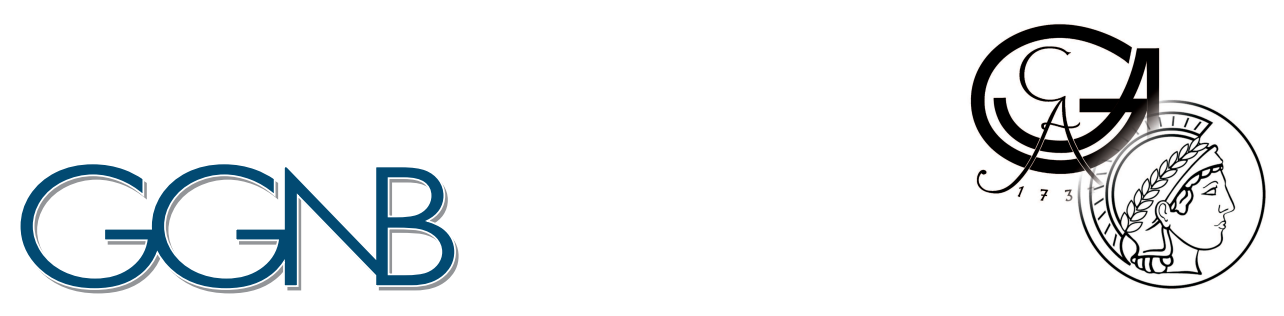

\title{
Molecular profiling of presynaptic docking sites
}

\author{
Dissertation \\ for the award of the degree \\ "Doctor rerum naturalium" (Dr. rer. nat.) \\ Division of Mathematics and Natural Sciences \\ of the Georg-August-Universität Göttingen
}

submitted by

Anne Janina Boyken

from

Oldenburg, Germany

Göttingen, 2011 


\section{Members of the Thesis Committee:}

\section{Prof. Dr. Reinhard Jahn $\left(1^{\text {st }}\right.$ Reviewer $)$}

Department of Neurobiology, Max Planck Institute for Biophysical Chemistry

\section{Prof. Dr. Ralf Ficner ( $2^{\text {nd }}$ Reviewer)}

Department of Molecular Structural Biology, GZMB, University of Göttingen

\section{Prof. Dr. Michael Thumm}

Department of Biochemistry II, University of Göttingen

Date of the oral examination: 


\section{Declaration of Authorship}

\section{Declaration of Authorship}

Hereby, I confirm that I have created this work "Molecular profiling of presynaptic docking sites" entirely on my own and that I have only used the sources and materials cited.

Göttingen, 30th of May 2011

Anne Janina Boyken 
Dedicated to 298 white wistar rats, who gave their brains for this thesis. 



\section{Abstract}

In presynaptic nerve endings, exocytosis of synaptic vesicles is restricted to specialized areas of the plasma membrane, called active zones, that are distinguished by electron-dense material. Here synaptic vesicles attach to the release sites (docking) and are then activated (priming) before undergoing calcium-dependent exocytosis, releasing their neurotransmitter content into the synaptic cleft. Many of the key players of the presynaptic exocytotic machinery are known, and also the major scaffold proteins of the active zone have been identified. However, the precise molecular composition of the sites at which vesicles dock remains to be elucidated. Proteomic approaches to identify protein components of these sites are challenging because of difficulties in purifying these sites. Most importantly it has been very difficult to separate presynaptic membranes from postsynaptic membranes and the postsynaptic protein scaffold. Here we report about a new procedure allowing for an almost quantitative separation of preand postsynaptic membrane fractions. The procedure involves mild proteolysis resulting in the cleavage of the adhesion molecules connecting pre- and postsynaptic membranes, followed by gradient centrifugation, lysis of the presynaptic compartment, separation of free and docked vesicles, and immunoisolation using antibodies specific for synaptic vesicle proteins as the final purification step. Using quantitative proteomics we then compared the protein composition of free and docked vesicles. In the latter fraction we detected all major active zone proteins. In addition we identified many ion channels and transporters, cell adhesion molecules and plasma membrane-specific signaling proteins that have been reported to be involved in synaptic transmission. Only very few postsynaptic proteins or proteins derived from other organelles (except of mitochondria) were detected. The docked vesicle fraction contained more than 30 previously uncharacterized proteins, many of which are predicted to contain single or multiple transmembrane domains. Preliminary characterization of one of the new membrane proteins using a newly generated antibody revealed specific localization to presynaptic nerve terminals, raising the possibility that the protein is involved in presynaptic function. Additionally, this new procedure was used to quantify changes in the presynaptic proteome as a result of effector treatment. The Rab effector GDI efficiently removed Rab proteins from the docked vesicle 
VIII

fraction, but no other significant changes were observed among the remaining 500 identified proteins in the docked vesicle fraction. 


\section{Contents}

Abstract

Contents IIX

$\begin{array}{lll}\text { List of Figures } & \text { XIII }\end{array}$

List of Tables

List of Abbreviations $\quad$ XV

1 Introduction 1

1.1 Key Events of Synaptic Vesicle Exocytosis . . . . . . . . . . . . . . . . 1

1.1 .1 Docking . . . . . . . . . . . . . . 2

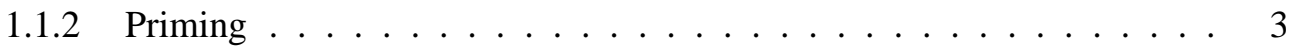

1.1 .3 Fusion . . . . . . . . . . . . . . . . . 3

1.2 Molecular Organization of the Active Zone . . . . . . . . . . . . . 4

1.2.1 Core Active Zone Proteins . . . . . . . . . . . . . . . . . . 5

1.2.2 Proteins involved in Synaptic Exocytosis . . . . . . . . . . . 8

1.3 Excitatory and Inhibitory Synapses . . . . . . . . . . . . . . . . . . 9

1.4 Synapse Proteomics . . . . . . . . . . . . . . . . . . . . . . 10

1.4.1 Proteomic Analysis of Synaptic Subdomains and Complexes . . . . . 10

1.4.2 Comparative and Quantitative Mass Spectrometry . . . . . . . . . . . 11

1.4 .3 Presynaptic proteomics . . . . . . . . . . . . . . . 13

1.5 Aims of this Work . . . . . . . . . . . . . . 14

2 Material \& Methods 15

2.1 Materials . . . . . . . . . . . . . . . . . 15

2.1 .1 Chemicals . . . . . . . . . . . . . . . 15

2.1 .2 Enzymes . . . . . . . . . . . . . . . . 16

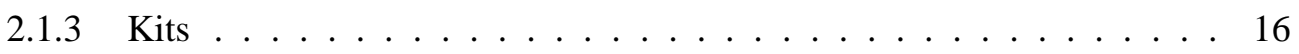


2.1 .4 Antibodies . . . . . . . . . . . . . . . . . . 16

2.1.5 Buffers and media $\ldots \ldots \ldots \ldots \ldots \ldots$

2.1.6 Mammalian cell lines and bacterial strains . . . . . . . . . . . . . 18

2.1 .7 DNA constructs . . . . . . . . . . . . . . . . . 18

2.2 Methods . . . . . . . . . . . . . . . . . . . . . . 19

2.2.1 Molecular Biology Methods . . . . . . . . . . . . . . . . . 19

2.2.1.1 Molecular Cloning . . . . . . . . . . . . . . . . . . . . 19

2.2.1.2 Protein Expression . . . . . . . . . . . . . . . 19

2.2.1.3 Protein Determination . . . . . . . . . . . . . . . 20

2.2.2 Cell Biological Methods . . . . . . . . . . . . . . . . . . 20

2.2.2.1 Cell Culture . . . . . . . . . . . . . . . 20

2.2.2.2 Transient Transfection . . . . . . . . . . . . . . . . 20

2.2.2.3 Immunofluorescent Staining . . . . . . . . . . . . . . 21

2.2.2.4 Image acquisition and processing . . . . . . . . . . . 21

2.2 .3 Biochemical Methods . . . . . . . . . . . . . . . 22

2.2.3.1 SDS-PAGE and Western Blotting . . . . . . . . . 22

2.2.3.2 Protein Extraction with Triton-X-114 . . . . . . . . 22

2.2.3.3 Preparation of Immunobeads . . . . . . . . . . . . . . 23

2.2.3.4 Preparation of Synaptosomes . . . . . . . . . . . . . 23

2.2.4 Protease Treatment of Synaptosomes . . . . . . . . . . . . . . . 24

2.2.4.1 Separation of Protease Treated Synaptosomes from the PSD . 24

2.2.4.2 Immunoisolation of Docked Vesicles from Protease treated Synaptosomes ................... 25

2.2.5 Mass Spectrometry Methods . . . . . . . . . . . . . . . 25

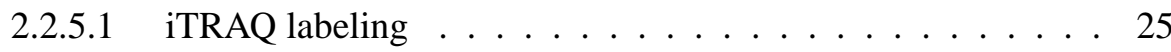

2.2.5.2 SCX Fractionation . . . . . . . . . . . . . 26

2.2.5.3 Mass Spectrometry and Quantification . . . . . . . . . 27

2.2.5.4 Data Normalization . . . . . . . . . . . . . . . . 27

2.2 .6 Rab Extraction Assay . . . . . . . . . . . . . . . . . . . . 27 27

2.2.7 Electron Microscopy . . . . . . . . . . . . . . . . . . . . 28

3 Results 29

3.1 Establishment of a protocol for the isolation of presynaptic membrane fractions 29

3.1.1 Removal of the postsynaptic density from synaptosomes . . . . . . . 30

3.1.1.1 Optimization of the protease treatment and separation of preand postsynaptic compartments . . . . . . . . . . . 30 
3.1.1.2 Separation of pre- and postsynaptic compartments ... . . 34

3.1.2 Isolation of a fraction enriched in docked vesicles . . . . . . . . . . . 36

3.1.2.1 Lysis of synaptosomes . . . . . . . . . . . . . . 36

3.1.2.2 Immunoisolation of docked vesicles . . . . . . . . . . . . 40

3.2 Quantitative comparison of a docked and free vesicle proteome . . . . . . . . 44

3.2.1 Synaptic vesicle proteins . . . . . . . . . . . . . . . . . 45

3.2.2 Active zone proteins . . . . . . . . . . . . . . . . . 46

3.2.3 SNARE proteins and trafficking molecules . . . . . . . . . . 48

3.2.4 Transporter, channel proteins and receptors . . . . . . . . . . . . . 49

3.2.5 Adhesion and cell surface molecules . . . . . . . . . . . . . . 51

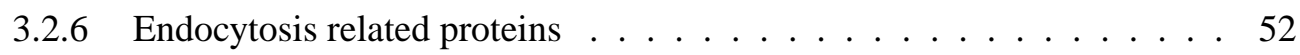

3.2.7 Cytoskeletal and associated proteins . . . . . . . . . . . . . 53

3.2 .8 Signaling molecules . . . . . . . . . . . . . . . . . 54

3.2 .9 Unknown proteins . . . . . . . . . . . . . . . . . . 5 57

3.3 Effect of the Rab GTPase modulator GDI on synaptic vesicle docking . . . . . 59

3.3.1 Removal of Rab3 from the vesicle membrane does not alter vesicle attachment to the plasma membrane . . . . . . . . . . . 59

3.3.2 GDI treatment does not remodel the protein composition of the active

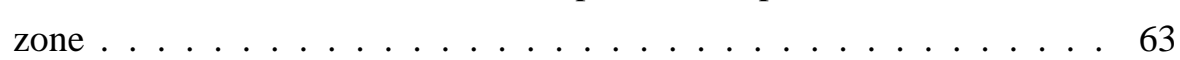

\begin{tabular}{ll}
$3.4 \mathrm{JB} 1$ is a novel transmembrane protein localized at presynaptic nerve terminals . 65 \\
\hline
\end{tabular}

3.4.1 Identification and characterization of JB $1 \ldots \ldots \ldots$

3.4.2 JB1 is a transmembrane synaptic protein . . . . . . . . . . 66

3.4.3 JB1 is associated with presynaptic structures $\ldots \ldots \ldots$

4 Discussion 73

4.1 A novel protocol to separate pre- and postsynaptic compartments . . . . . . . 73

4.1 .1 Method . . . . . . . . . . . . . . . . . . . . 75

4.1.2 Presynaptic proteome . . . . . . . . . . . . . . . . . 77

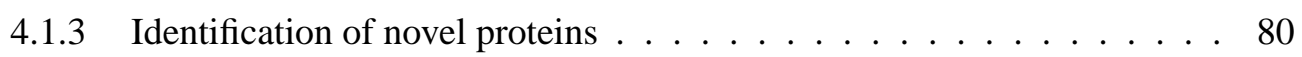

4.1.4 Versatility/usage of the method . . . . . . . . . . . . . . . 80

4.2 Investigation of proteomewide changes in synaptic vesicle docking site upon treatment with effectors . . . . . . . . . . . . . . . . . . . . . . . . . . . . .

4.3 Conclusion and outlook . . . . . . . . . . . . . . . . . 84

References

\begin{tabular}{ll} 
Acknowledgements & 113 \\
\hline
\end{tabular} 
Curriculum vitae

115

Appendix 


\section{List of Figures}

$1.1 \quad$ Architecture of an Excitatory Synapse . . . . . . . . . . . . . . 1

1.2 Morphology of docked synaptic vesicles . . . . . . . . . . 2 2

1.3 Morphology of presynaptic release cites . . . . . . . . . . . . . . 4

1.4 Model of Rim in the presynaptic release machinery . . . . . . . . . . . . 6

1.5 Molecular model of a synaptic vesicle . . . . . . . . . . . . . . . . . . . . . 11

1.6 Electron micrograph of a synaptosome .............. 13

3.1 Purification scheme for the separation of pre- and postsynaptic membranes . 31

3.2 Optimization of synaptosomal proteolytic treatment . . . . . . . . 33

3.3 Effect of protease treatment on various protein groups . . . . . . . 35

3.4 Separation of pre- and postsynaptic membranes by gradient centrifugation . . 37

3.5 Immunofluorescent staining of synaptosomes . . . . . . . . . . . . . 38

3.6 Lysis of synaptosomes . . . . . . . . . . . . . . . . . . 39

3.7 Synaptic mitochondria co-migrate with docked vesicles . . . . . . . . . 41

3.8 Immunoisolation of docked and free vesicles . . . . . . . . . . . . 43

3.9 Proteins identified by iTRAQ analysis . . . . . . . . . . . . . . 45

3.10 Schematic synapse composed of the iTRAQ data . . . . . . . . . . 58

3.11 GDI effect on lysed synaptosomes . . . . . . . . . . . . . . . . . . . 60

3.12 GDI effect on the docked vesicle fraction . . . . . . . . . . . . 62

3.13 Proteomic changes induced by GDI . . . . . . . . . . . . . . . . . . 64

3.14 Structure and expression of JB1 . . . . . . . . . . . . . . . . . . 67

3.15 Tissue and subcellular distribution of JB1 . . . . . . . . . . . . . . . 69

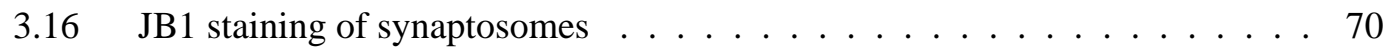

3.17 Immunofluorescence analysis of JB1 in rat hippocampal neurons . . . . . . 72

4.1 Overlap between proteomic datasets derived from different presynaptic fractions -published information . . . . . . . . . . . . . . . . 79 


\section{List of Tables}

1.1 Active zone interactions . . . . . . . . . . . . . . 8

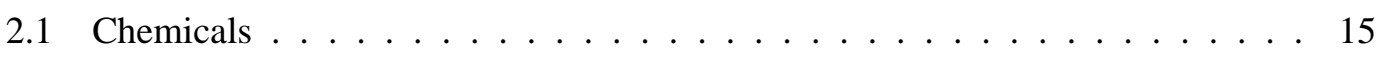

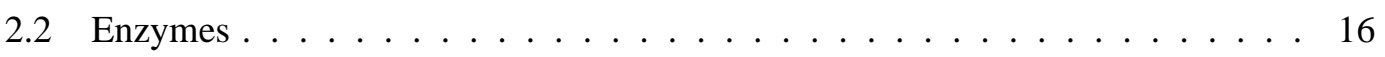

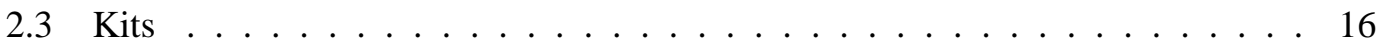

2.4 Antibodies . . . . . . . . . . . . . . . . . . . 17

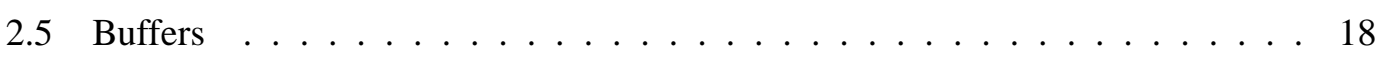

3.1 Synaptic vesicle proteins $\ldots \ldots \ldots \ldots \ldots$. . . . . . . . . . . . . . . . . .

3.2 Active zone proteins . . . . . . . . . . . . . . . . 48

3.3 SNARE proteins and trafficking molecules . . . . . . . . . . . . . . 49

3.4 Transporter, channel proteins and receptors . . . . . . . . . . . . 50

3.5 Adhesion and cell surface molecules . . . . . . . . . . . . . . 52

3.6 Endocytosis related proteins $\ldots \ldots \ldots \ldots \ldots \ldots$

3.7 Cytoskeletal and associated proteins $\ldots \ldots \ldots \ldots \ldots 5$

3.8 Signaling molecules $\ldots \ldots \ldots \ldots \ldots \ldots \ldots \ldots$

3.9 Unknown proteins . . . . . . . . . . . . . . . . . . . 57 


\title{
List of Abbreviations
}

\author{
AZ ......... Active zone \\ BLAST ....... Basic Local Alignment Search Tool \\ BSA $\ldots \ldots \ldots$ bovine serum albumin \\ CASK ....... calcium/calmodulin-dependent serine protein kinase \\ ERC2 $\ldots \ldots \ldots$ ELKS/Rab6-interacting/CAST family member 2 \\ GABA ....... $\gamma$-aminobutyric acid \\ GAP43 ...... Growth-Associated Protein 43 \\ GDI ........ GDP dissociation inhibitor \\ GluR1 ....... glutamate receptor, ionotropic, AMPA1 \\ HRP ....... Horse raddish peroxidase \\ IEF $\ldots \ldots \ldots$ isoelectric focusing point \\ iTRAQ ....... isobaric tag for relative and absolute quantitation \\ NCAM ....... neuronal adhesion molecule 1 \\ NCBI ....... National Center for Biotechnology Information \\ $\mathrm{NCX} \ldots \ldots . . \mathrm{Na}^{+} / \mathrm{Ca}^{2+}$ \\ NHE1 ....... sodium/hydrogen exchanger 1 \\ NR1 ........ glutamate receptor, ionotropic, N-methyl D-aspartate 1 \\ NSF $\ldots \ldots \ldots N$-Ethylmaleimide sensitive factor \\ PBS ......... phosphate buffered saline \\ PMCA ...... plasma membrane $\mathrm{Ca}^{2+}$ - ATPase \\ PSD95 $\ldots \ldots$ postsynaptic density protein 95 \\ SCX ........ Strong cation-exchange chromatography \\ SDHA ....... Succinate dehydrogenase complex, subunit A, flavoprotein \\ SDS-PAGE ... Sodium dodecyl sulfate polyacrylamide gel electrophoresis \\ SMART ...... Simple Modular Architecture Research Tool \\ SNARE ....... soluble NSF attachment protein \\ SPM ........ Synaptic plasma membrane
}


SV .......... Synaptic vesicle

TEMED ...... N, N, NŠ, NŠ Tetramethylethylenen-diamine

VGlut $1 \ldots \ldots$ vesicular glutamate transporter 1 


\section{Introduction}

\subsection{Key Events of Synaptic Vesicle Exocytosis}

Synapses are the fundamental units of neuronal networks, representing the sites of information transfer between neurons. Structurally, synapses are characterized by their asymmetric organization consisting of a presynaptic nerve terminal containing synaptic vesicles (SV), a synaptic cleft, and a postsynaptic signaling complex called the postsynaptic density (PSD). At the synapse, an arriving electrical signal is converted into a chemical signal. Precisely, action potential depolarization of the presynaptic plasma membrane induces calcium channel opening and calcium influx into the nerve terminal that triggers exocytosis of neurotransmitterfilled synaptic vesicles. Neurotransmitter molecules then diffuse across the synaptic cleft and bind to postsynaptic receptors triggering signal transduction cascades at the postsynaptic site. This neurotransmitter release is restricted to specialized presynaptic membrane compartments called active zones, where synaptic vesicles undergo a temporally and spatial coordinated 3step mechanism consisting of docking, priming and fusion (Fig. 1.1).

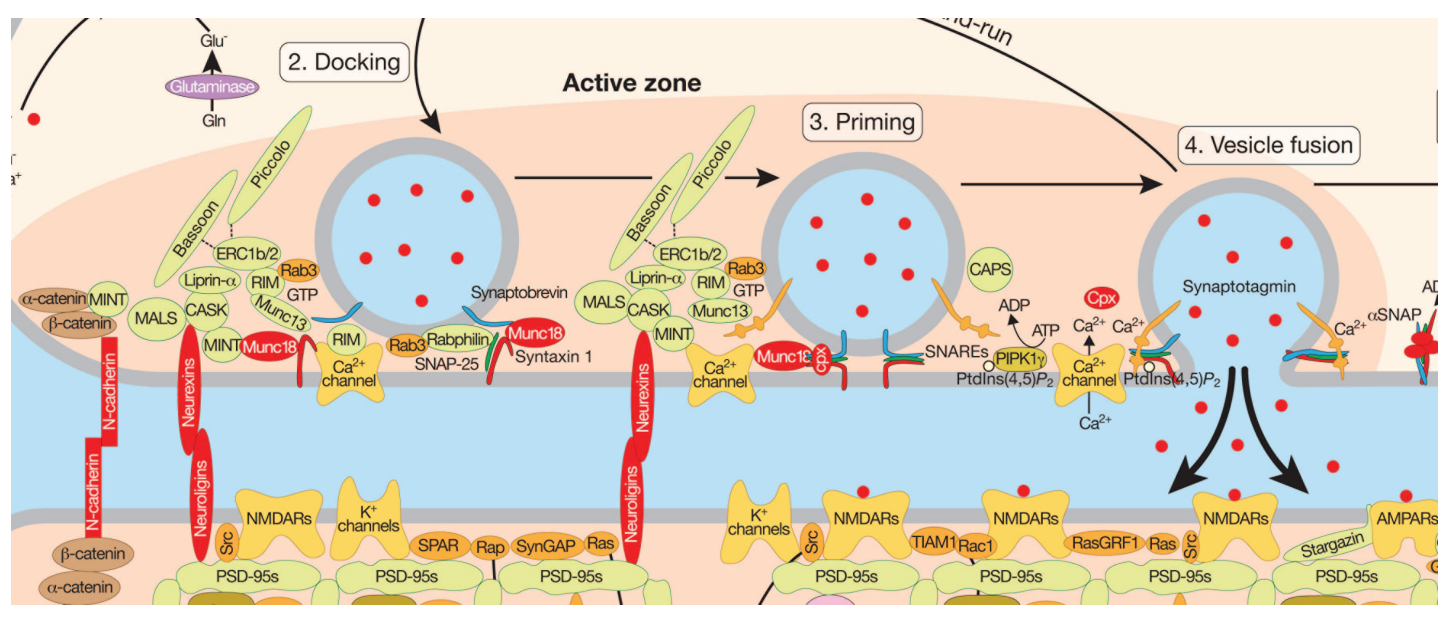

Figure 1.1: Image modified from [1]. The key stages during synaptic transmission at the active zone are the translocation and docking of SVs to the plasma membrane, priming, vesicle fusion and synaptic vesicle recycling. 


\subsubsection{Docking}

The initial step of neuronal exocytosis requires the recruitment and localization of synaptic vesicles to the presynaptic active zone, a process termed docking. Docking is traditionally defined as the morphological attachment of synaptic vesicles to the plasma membrane. In electron micrographs, these vesicles appear without a measurable distance to presynaptic membrane (Fig. 1.2) [2, 3]. Generally, docking is thought to be the preceding step before vesicles gain fusion competence. As there is currently no defined relationship between this docking structure and function, the molecular background of docking can only be inferred from morphological phenotypes. The prime candidate believed to function as a docking factor is Munc18. A severe phenotype of a reduced vesicle docking was observed in Munc18-deficient chromaffin cells [4, 5], in neurons [6] and at neuromuscular junctions of $C$. elegans [7]. It is believed, that Munc18s role in docking is highly dependent on the interaction with the "closed" conformation of syntaxin 1 [8, 9] that occludes the binding site for the cognate SNARE partners and therefore inhibits SNARE complex assembly.

On the vesicular side, Synaptotagmin has been suggested to anchor vesicles in chromaffin cells by binding to the syntaxin-1/SNAP25 complex, an acceptor for subsequent synaptobrevin binding [10,11]. This docking role for synaptotagmin was also reported for invertebrate synapses [12, 13, 14]. Rab proteins have also been suggested to influence docking. A docking phenotype in secretory cells was observed for Rab3 [15, 16] and Rab27 [17, 18] proteins. However, a similar phenotype for Rab3 in synapses could not be proven [19, 20]. The redundancy of Rab proteins might account for the missing phenotype in synapses, especially since Rab3 and Rab27 share overlapping functions and possibly compensate each other [21].

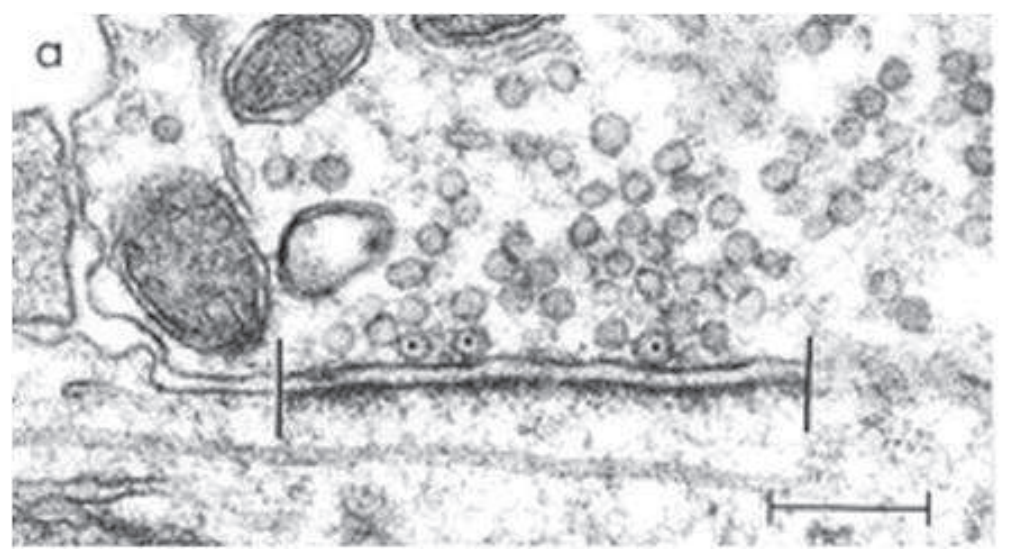

Figure 1.2: Image modified from [22]. Docking of synaptic vesicles at the active zone. Active zones are marked by vertical bars, and docked synaptic vesicles (within $10 \mathrm{~nm}$ of the presynaptic membrane), with black dots. 


\subsubsection{Priming}

Priming is defined as the process that makes a (docked) vesicle fusion competent such that it can undergo exocytosis upon calcium influx. In contrast to docking, priming is primarily based on electrophysiological observations. Primed vesicles constitute the readily releasable pool of vesicles (RRP) that are released fast during a stimulus and that can be assayed by applying an emptying stimulus [23, 24, 25]. This functionally defined readily releasable pool essentially coincides with the morphologically defined docked vesicle pool, making it difficult to resolve their relationship and raising the question if priming really is an independently regulated process. However, based on observations where the number of docked vesicles differs from the number of primed vesicles, docking and priming are believed to be separate steps [26, 27]. Nevertheless, perturbations of proteins involved in priming and fusion often also impair vesicle docking, suggesting that these processes are sequentially interlinked [8, 2].

Unlike the less resolved process of docking, the molecular mechanism that underlies vesicle priming is better understood and requires the formation of the trimeric SNARE complex of syntaxin/SNAP25/synaptobrevin [28, 29, 30] and its interaction with Munc18 [31, 32]. SNARE-assembly starts by formation of a four-helix bundle of the SNARE-domains residing on two opposing membranes. This helical bundle zippers up from the $\mathrm{N}$ - to $\mathrm{C}$-terminus, forming a trans-SNARE complex. The partial if not complete assembly of this trans-SNARE complex between the synaptic vesicle and the plasma membrane then bridges the fusing membranes, bringing them in close proximity [33, 34]. In addition to forcing the synaptic vesicle into a fusiogenic state, premature fusion has to be prevented, so that exocytosis only takes place when calcium enters the cell. In this respect, complexin has been suggested to regulate fusion by binding to the zippered SNARE complex, "clamping" it in an activated but frozen state [35, 36, 37]. How binding of complexin to the SNARE complex regulates the probability of SV fusion is controversial and to date not fully understood [38].

Aside from the components of the fusion machinery, additional proteins that regulate priming have been identified. Munc13 is the best characterized priming factor (see section 1.2.1), influencing the size of the RRP in chromaffin cells [39] and in neurons [40].

\subsubsection{Fusion}

After membrane docking and priming, fusion is initiated by the influx of calcium through voltage-gated calcium channels at the plasma membrane. As a consequence, calcium binding to the synaptic vesicle protein synaptotagmin triggers the molecular mechanism of membrane fusion [41, 42, 43]. Synaptotagmin contains two cytoplasmic C2 domains (C2A and C2B) that bind calcium ions enabling them to interact with phospholipids in the plasma membrane 
[44, 45]. Synaptotagmin simultaneous interacts with the target membrane SNARE proteins upon binding calcium ions [46, 47], therefore synaptotagmin influences both lipid bilayers and SNARE proteins. These calcium-dependent interactions are thought to control transitions in the fusion machine. Synaptotagmin is thought to displace complexin followed by a complete zippering of the SNARE complex and fusion of the membrane [37, 48, 49]. According to the established mechanistic model, membrane fusion is driven by the free energy that is released upon formation of the fully zippered SNARE complex [50]. As the membrane fuses, SNAREs are transformed to a cis-complex, where the proteins reside in the same membrane. SNARE complexes are then dissociated by NSF and the soluble NSF attachment proteins (SNAPs) [51].

\subsection{Molecular Organization of the Active Zone}

In principle, active zones provide a molecular platform for the arriving vesicle, localizing them in close proximity to the plasma membrane (docking) and preparing them for exocytosis (priming). Already in the early 60ies actives zones were visualized as electron dense particles in electron micrographs that are precisely aligned opposite to the postsynaptic density [52, 53]. Since then, the knowledge about AZ morphology has advanced tremendously to the point of detailed 3D tomographs [54, 55, 56]. These structures revealed, that at the morphological level the active zone is identified by the presence of synaptic vesicles linked to each other and to the plasma membrane by a filamentous network (see Fig. 1.3). Unlike AZ structure, the knowledge
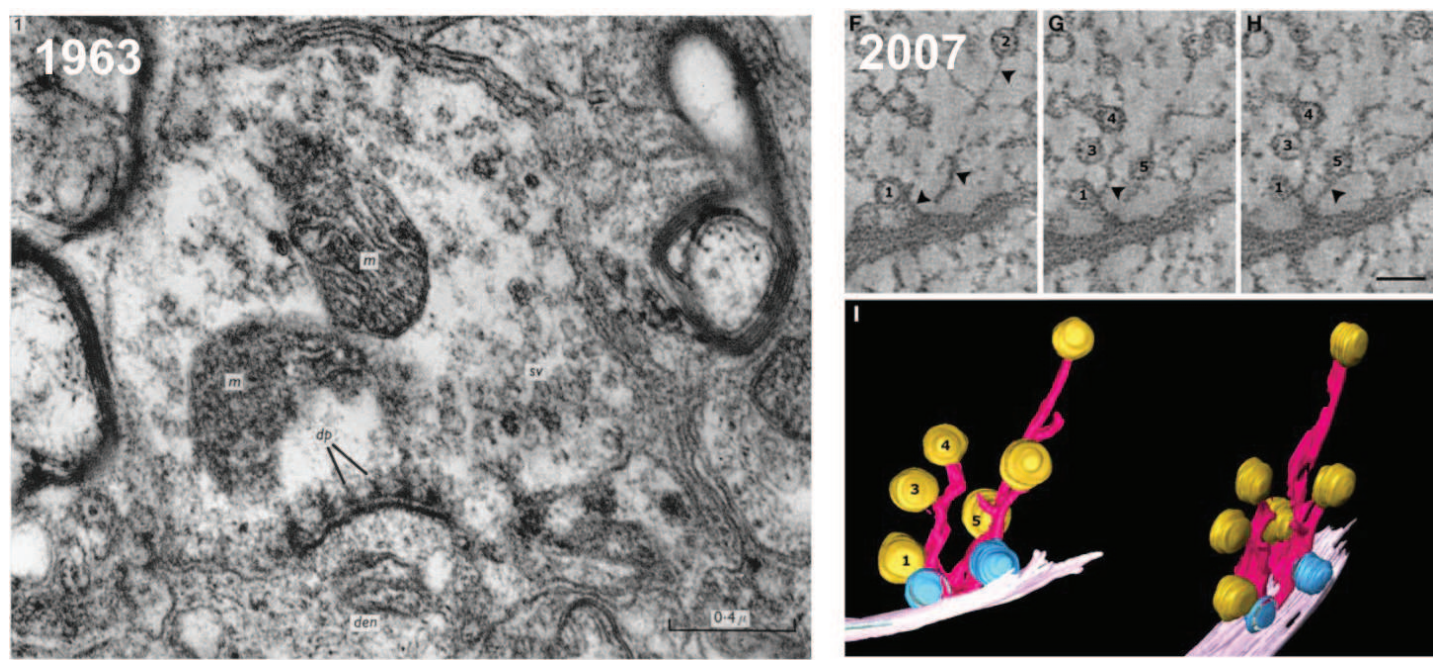

Figure 1.3: 1963: Image modidfied from [52]. Synapse of spinal cord showing regularly arranged dense projections in presynaptic processes. m: mitochondrium, dp: presynaptic dense projections, den: dendrite. 2007: Image modified from [55]. F-H: Example of a filament contacting several SVs. I: 3D reconstruction of the filaments (pink) and the adjacent SVs. 
about the molecular basis that mediates and regulates presynaptic events has advanced much slower and remains to be fully elucidated. Since its discovery in the 1960s, only a few protein families have been identified to be specifically associated with the active zone.

\subsubsection{Core Active Zone Proteins}

\section{Munc13}

Proteins of the Munc13 family were the first identified components of the presynaptic active zone. These proteins have originally been identified in a genetic screen for uncoordinated movements in C.elegans (UNC13) [57]. The mammalian homologue of unc13, Munc13, has 3 isoforms that are expressed in the nervous system [58] and that share a common multidomain structure consisting of a C1, C2 (2x), C2B, C2C and a central MUN domain [59]. Several studies have suggested that Munc13 proteins are involved in synaptic vesicle priming [60, 61]. At the physiological level, a deletion of Munc13-1 in primary hippocampal neurons [40] as well as in neuromuscular junctions of C.elegans [62] and Drosophila [63] impairs neurotransmitter release as a result of defects in the size of the readily releasable vesicle pool. At the molecular level, Munc13 proteins interact with multiple proteins including DOC2 [64], calmodulin [65], spectrins [66], Rim [67, 68], syntaxin [69, 70, 71] and Munc18 [72]. Munc13 additionally forms inactive homodimers that can be relieved by binding to Rim [68, 73]. It has emerged recently that the interaction with syntaxin and Munc18 is the main mechanism for Munc13 in priming. It has been suggested that a weak interaction of the MUN domain with the SNARE motif of the closed syntaxin-Munc18 complex accelerates opening of syntaxin1 and thus SNARE complex assembly [72]. Munc13 function is additionally calcium regulated via its C2B domain [74, 71].

\section{Rim}

In the mammalian system, there are 7 Rim isoforms encoded by 4 genes [75, 76, 77]. Initially identified as Rab3-interacting proteins [78], these proteins have emerged to be the central organizers of the active zone. Rim has been shown to have multiple roles in neurotransmitter release including docking [79, 80, 81], priming [82, 83], calcium channel localization [84, 80] and plasticity [77, 85]. Containing various domains (Zn-finger, PDZ, C2A, C2B and a prolinerich PxxP motif) [75], Rims can interact with other active zone components e.g. Piccolo [86], ERCs [87, 88, 89], Liprins [85] and Munc13 [67]. Additionally, Rims also bind to the synaptic vesicle proteins Rab3 [78] and synaptotagmin [77], to Rim-BPs [75] and to calcium channels [77]. A global understanding of how a single protein can translate such a diverse range of interaction partners into physiological function has not been accomplished. However, some of 


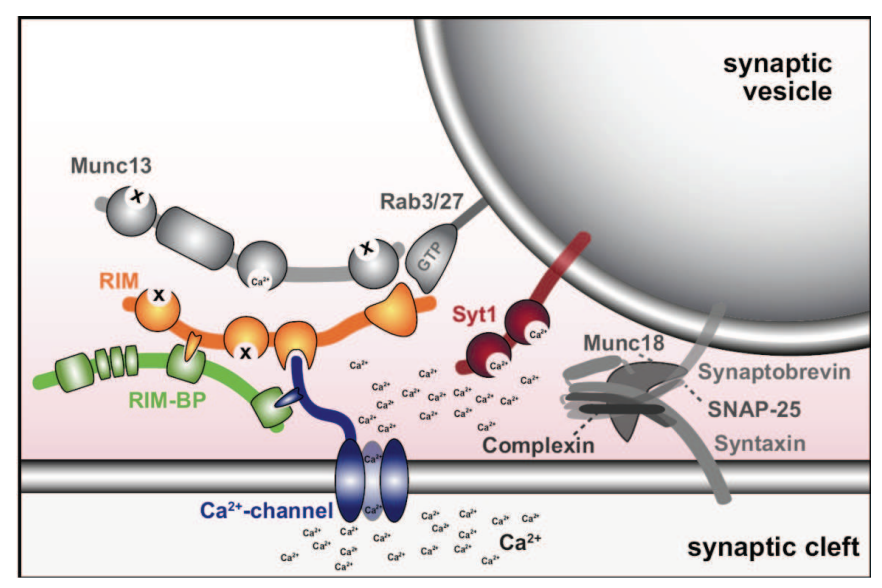

Figure 1.4: Image modidfied from [84]. Model illustrates the N-terminal priming complex of Rim with Rab3 and Munc13 and localization of calcium channels at the active zone via a direct interaction with the PDZ domain.

these molecular interactions have been unraveled and could be assigned to a specific synaptic function. For example, the N-terminal Zn-finger domain binds to Rab3 and this interaction is suggested to be involved in recruitment/docking of vesicles to the membrane [79]. This interaction presumably leads to an additional or sequential binding of Rim to Munc13, that activates priming by relieving the autoinhibitory homodimerization of Munc13 [90]. Furthermore, the Rim PDZ domain has been shown to directly interact with calcium channels, tethering them in close proximity to the release sites [84]. Thus, Rim modulates sequential steps in synaptic vesicle exocytosis through serial protein-protein interactions (see Fig. 1.4).

\section{ERCs}

ERCs [88], also known as ELKS [91] (ERC1) or CAST [87] (ERC2) are active zone components that were independently found at the same time as interaction partners for Rim in a yeast-two-hybrid screen [88] and complexed with Rim and Munc13 in PSD preparations [87]. ERCs are composed of 4 coiled-coil domains that can bind to piccolo, bassoon and liprins [92, 93] and a C-terminal-consensus binding motif that interacts with the PDZ-domain of Rim [88, 87, 89]. Hence ERCs can bind, similar to Rim, to many active zone components providing a platform for the release machinery [92]. ERCs appear to function downstream of synaptic vesicle docking [94, 95, 96], yet their precise function in neurotransmitter release is controversial. Two opposing theories, one suggesting an essential role in exocytosis affecting the Rim-Munc13 pathway [92, 94] and one establishing ERC as a negative regulator, restricting release at inhibitory synapses [96] are currently considered. 


\section{Piccolo and Bassoon}

Piccolo and bassoon represent the largest active zone-specific proteins and are structurally related molecules. They are 530 and $420 \mathrm{kDa}$ in size and contain multiple domains including two $\mathrm{N}$-terminal zinc finger motifs, three coiled-coiled regions and, in the case of Piccolo, a PDZ and two C2 domains [97, 98, 99]. Just like Rims, Munc13s and ERCs these proteins closely interconnect with other members of the AZ (e.g., ELKs [92], Rims [86] and Liprin [100]), but piccolo additionally binds to proteins involved in the regulation of actin and $\mathrm{SV}$ dynamics (GIT1 [101], Abp1 [102], profilin [99], and PRA-1 [98]). Having a size of more than $400 \mathrm{kDa}$, addressing protein function of these molecules with conventional knock down strategies is elusive [103]. Additionally, the high structural similarity and overlap of binding partners possibly causes functional redundancy. Nevertheless piccolo and bassoon are suggested to be involved in the formation of active zones from precursor vesicles early in synaptogenesis [104, 105]. Bassoon additionally exhibits a unique structural role in the attachment of ribbon synapses [106, 107], while piccolo's role has not been completely resolved yet. On the one hand it is believed that piccolo functions as a negative regulator of exocytosis by -either direct or indirectmodulating synapsin dynamics that affects the recruitment of synaptic vesicles from the reserve pool to the readily releasable pool [108]. On the other hand it is thought that piccolo does not directly participate in vesicle exocytosis, but has a significant role in maintaining vesicle clusters [109].

\section{$\alpha$-liprins}

$\alpha$-liprins are the least characterized active zone components. Although their presence is not restricted to active zones, $\alpha$-liprins are considered an integral part of presynaptic release site [110]. These proteins were originally identified as LAR interaction partners and exist in 4 structurally homologous isoforms, consisting of several N-terminal coiled-coil domains and a C-terminal liprin homology (LH) domain [100]. As the other active zone proteins, liprins can directly interact with Rim [85], ERC1 [93, 111] and CASK [112]. Liprins are thought to play a crucial role in active zone organization [113, 111]. Precisely, $\alpha$-liprins are thought to be upstream effectors of Rim, possibly by localizing Rim to active zones [85]. Evidence that liprins and Rim act in the same pathway can be provided by the similarity of the morphological and physiological phenotypes [81, 114]. It has also been suggested that binding to ERC in turn influences the presynaptic localization of liprins [93, 111].

\section{CASK, Mint, MALS}

CASK, MALS (Veli) and Mint form a ternary complex [115, 116] that possibly occurs on both sides of synaptic junctions [117, 112, 118, 119]. Presynaptically, proteins of this complex 
interact with neurexin (CASK)[117] and Munc18 (Mint) [120] while the assembled complex associates with $\alpha$-liprins [112]. The function of this complex in synaptic transmission remains elusive, but there are indications that it is involved in replenishing the readily releasable pool from the reserve pool of synaptic vesicles at the active zone and that liprin might participate in this function [112].

\begin{tabular}{|l|l|l|}
\hline Protein & Interaction partner & Reference \\
\hline ERC2 & bassoon and piccolo & {$[92]$} \\
& RIM and Munc13 & {$[[87]$} \\
& $\alpha$-liprins & {$[93]$} \\
\hline Rim & Rab3 & {$[78]$} \\
& Munc13 & {$[67]$} \\
& N- and P/Q-type $\mathrm{Ca}^{2+}-$ channels & {$[[84]$} \\
& $\alpha$-liprins & {$[85]$} \\
\hline CASK & liprin- $\alpha 2$ & {$[112]$} \\
& Mint1 and MALS & {$[115]$} \\
\hline
\end{tabular}

Table 1.1: Compendium of known interactions between active zone components.

\subsubsection{Proteins involved in Synaptic Exocytosis}

\section{Adhesion Molecules}

Although not classified as true active zone components, cell adhesion molecules participate in the function and plasticity of synapses aside from their structural role [121]. As an example, the presynaptic adhesion molecules $\alpha$-neurexins have been suggested to regulate calcium channel function, because a loss of these molecules resulted in a decrease of whole cell calcium currents [122]. In addition, neurexin can bind to the active zone protein CASK [117]. There is also evidence for a role of NCAMs and cadherins in synaptic plasticity, but these adhesion molecules reside on both sites of the synapse, which makes it difficult to unravel only their presynaptic function. Cadherins are thought to contribute to synaptic plasticity by interacting with catenins. These complexes are known to regulate postsynaptic AMPAR trafficking and are involved in dendritic spine formation. On the presynaptic site, $\mathrm{N}$-cadherins are localized close to the active zone and have been demonstrated to influence synaptic vesicle release at glutamatergic synapses [123].

\section{Cytoskeletal Elements}

Cytoskeletal components, especially actin, are highly enriched at synapses. Actin does not only 
define synapse morphology, it is additionally thought to be involved in the regulation of synaptic transmission. However, the mechanisms of actin dynamics regulating synapse function is not completely understood. The actin cytoskeleton is suggested to function as a physical barrier in the nerve terminal that is necessary to maintain the required distance between different vesicle pools or opposing membranes. Thus, actin is thought to function as a negative regulator, restricting vesicle recruitment and fusion. However, actin also facilitates the delivery of synaptic vesicles from the reserve pool to the RRP through molecular motors and therefore positively influences the synaptic vesicle exocytosis (for review see [124]). Interestingly, presynaptic proteins such as the SV protein synapsin [125] or the active zone protein piccolo [C. Waites, data not published] can directly associate with the actin cytoskeleton, but the precise function of these interactions are not fully understood.

\subsection{Excitatory and Inhibitory Synapses}

The brain is composed of many different types of neurons that form very specific synapses. Already the earliest morphological studies proved that synapses are not equivalent, but exist in different types (Gray's type I and type II) [126]. With the current knowledge, it is well-known that in the central nervous system (CNS) synapses are either excitatory and inhibitory. These synapses differ in the identity of neurotransmitters, in receptor types at the postsynaptic site, and the ability to depolarize or hyperpolarize neurons. The majority of the synapses in the CNS are in fact excitatory synapses that mediate synaptic transmission by the neurotransmitter glutamate. Glutamate binds postsynaptically to the ionotropic N-methyl-D-aspartic acid (NMDA) receptor and the $\alpha$-amino-3-hydroxy-5-methyl-4-isoxazolepropionic acid (AMPA) receptor. Only a small portion of the synapses (10-20\%) are inhibitory synapses. Synaptic transmission at these synapses is dependent on gamma aminobutyric acid (GABA), which activates a family of GABA receptors on the postsynapt site.

Although the machinery of synaptic exocytosis is present in both types of synapses, surprisingly few proteins are common to all synapses, but are expressed in different isoforms. Recent evidence is suggesting that GABAergic and glutamatergic neurons express different isoforms of molecular components that regulate pre-and postsynaptic functions. On the postsynaptic site, neuroligins and gephyrins for example have been shown to be differentially expressed among synapse types [127, 128]. On the presynaptic site, Munc13s and synapsins have been suggested to play different roles in excitatory and inhibitory synapses [61, 129]. Interestingly, immunoisolated SVs specific for the different neurotransmitter do not differ significantly in their protein composition apart from the vesicular neurotransmitter transporter [130], indicating that SVs do not make the key difference in these synapses. 


\subsection{Synapse Proteomics}

\subsubsection{Proteomic Analysis of Synaptic Subdomains and Complexes}

Although the knowledge of the molecular components in the presynaptic nerve terminal remains limited, proteomic studies have produced a wealth of qualitative data so far. Such studies have contributed to the understanding of synaptosomes, synaptic sub-compartments such as the postsynaptic density or synaptic vesicles.

Almost a decade ago, the first large-scale proteomic analysis of the NMDA receptor complex was carried out, identifying more then 70 proteins in a single multi-protein signaling complex [131]. The identification of a physical and functional unit comprised of receptos, adaptors, signaling and cytoskeletal components was a novelty. It complemented and exceeded previous studies that were based on yeast-two-hybrid screens and additionally provided new insights into NMDA receptor function. Around the same time, major efforts started to identify postsynaptic proteins from the purified PSD fraction by large-scale proteomic analyses [132]. To date, several hundred proteins are identified reflecting the diversity and complexity of the postsynaptic density, among them are ion channels, scaffolding molecules, signaling and cytoskeletal elements, proteins involved in sorting and trafficking as well as protein synthesis [133, 134, 135]. Novel proteins identified by these proteomic studies were then followed up and integrated into the existing model of the postsynaptic density.

Another example that has been extensively analyzed by proteomic studies, is the synaptic vesicle [136, 137, 138] (see Fig. 1.5). In 2006, two independent studies comprehensively characterized the SV proteome and could identify $185[137]$ and $410[138]$ proteins, depending on sample preparation and subsequent mass spectrometric analysis. The large number of proteins detected on a organelle with an average diameter of $40 \mathrm{~nm}$ was surprising and gave rise to the necessity of additional quantitation methods to distinguish bona fide organellar proteins from those who are contaminating. Therefore, Takamori and co-workers performed a comprehensive Western blotting profiling of subcellular fractions to discriminate between proteins co-purified with synaptic vesicles, proteins distributed throughout all subcellular fractions and proteins that are depleted from the SV fraction. The principle of such a procedure is known as protein correlation profiling and was introduced to study the human centrosome [139]. Nowadays, due to the high sensitivity of mass spectrometers, protein correlation profiling has become almost indispensable to generate reliable subcellular proteomes. 


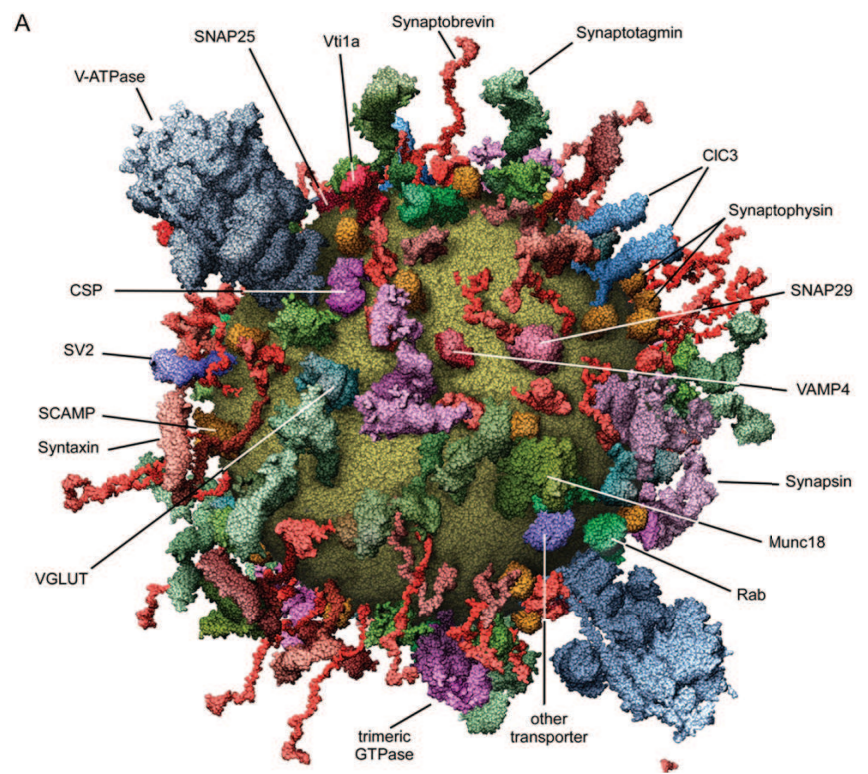

Figure 1.5: Image modidfied from [138] shows a 3D-model of an average synaptic vesicle. The quantitative description of a SV was generated by molecular, biophysical, electron microscopy, and modeling techniques.

\subsubsection{Comparative and Quantitative Mass Spectrometry}

Due to the increasing sensitivity of mass spectrometers, the necessity for quantification in addition to identification has emerged. The isolation procedure of subdomains or organelles merely enriches proteins, so that preparations may contain a considerable amount of contaminants. Instead of doing time consuming Western blotting profiling, quantitative mass spectrometry has moved into focus. Early approaches used a label-free quantitation based on peptide ion intensities that are correlated to the concentration of the peptides [139]. However, this method strongly depends on the stability of LC separation and MS analysis and is complicated when analyzing complex samples. Apart from protein correlation profiling, quantitative information of protein and protein complexes have a great prospect. Takamori and co-workers provided the first quantitative description of a organelle by quantifying all major SV proteins by time- and work-consuming Western blotting, taking purified proteins as a reference [138]. Nowadays, tools have been developed that enable quantitative measurements and comparisons of complex protein samples by using stable isotope labeling on either protein or peptide level.

\section{- ICAT}

Isotope-coded affinity tagging (ICAT) chemically labels reduced cysteinyl residues of the proteins [140]. It allows a relative quantification based on the enrichment of labeled 
peptides in the MS analysis. However, proteins containing no cysteine can not be quantified.

\section{- ITRAQ}

Isobaric tags for relative and absolute quantitation (iTRAQ) labels trypsin digested peptides at N-termini and lysine-residues [141]. A collision-induced dissociation of the labeled peptide generates signature ions, whose intensities are used to calculate the relative quantity of a protein. Labeling efficiency is an issue with this method and needs to be checked.

\section{- AQUA}

Absolute QUAntification (AQUA) is the only absolute quantification method. It is based on internal heavy isotope labeled peptide standards that are chemically synthesized and spiked into the sample [142]. The absolute quantity of native peptides can be calculated using mass spectrometric peak ratios. A major drawbacks of this method are high costs and the impracticalness of synthesizing large numbers of peptides to cover the desired proteome.

\section{- SILAC}

Stable isotope labeling with amino acids in cell culture (SILAC) is an in vivo approach that metabolically labels proteins during cell growth [143]. SILAC relies on the incorporation of stable isotopic nuclei that generate a light $(12 \mathrm{C}, 14 \mathrm{~N})$ or heavy $(13 \mathrm{C}, 15 \mathrm{~N})$ form of the amino acid into the proteins. The labeling efficiency with this method is nearly $100 \%$, but labeling of non-mitotic cells or tissue can not be achieved.

One of these methods, ICAT was first used to distinguish postsynaptic density specific proteins from co-purifying contaminants [134]. By correlation-profiling of synaptic membranes containing the PSD and isolated PSD fractions, a number of proteins were depleted, indicating that they were contaminants of the PSD preparation.

With AQUA, it is even possible to measure molar concentrations and relative stoichiometries of proteins within a sample. By using this method, absolute amounts of several key PSD proteins, e.g. glutamate receptor subunits, were measured for the first time [135].

An approach that involves the stable isotope reagent iTRAQ has a major advantage as it allows to analyze up to 8 samples simultaneously. Using this approach, a quantitative comparison of glutamatergic and GABAergic synaptic vesicles was done showing that the vesicular transporters are the only components essential for defining the neurotransmitter phenotype of a SV [130]. 
The study of synaptic regulation and function often involves post-translational modifications such as phosphorylation. In this respect, although not always quantitative, large-scale phosphoproteome analyses involving an affinity isolation step to enrich phosphopeptides have been carried out on synaptosome and PSD preparation [144, 145]. Impressively, nearly 1000 phosphorylated peptides from 287 proteins were identified from PSD preparation [145]. There are many more examples of quantitative proteomics that have been successfully applied to analyze proteomic changes, for example during brain development or in knockout mice models. All these analyses have contributed to the fundamental question of how a complex protein network drives synaptic function.

\subsubsection{Presynaptic proteomics}

The main advantage of synaptosomes, PSDs and SVs is that they can be isolated in high amounts and with a sufficient purity. Synaptosomes are generated during homogenization of brain material. The applied mechanical forces tear the nerve terminal apart from the axon, which then reseals to form a membrane enclosed giant organelle that includes the presynaptic release machinery, a large number of synaptic vesicles, mitochondria and cytosolic components. On the outside of the synaptosomal membrane, main parts of the postsynaptic density are attached through the transsynaptic scaffold (see Fig. 1.6). These artificial organelles can be isolated by density gradient centrifugation [146]. The postsynaptic density can be obtained from synaptosomes by extraction with Triton-X-100. The PSD remains as a detergent resistent, unsoluble fraction that can be collected by another round of gradient centrifugation. Synaptic vesicles are also isolated from synaptosomes, they are released by hypoosmotic shock and further fractionated by gradient centrifugation, controlled pore-glass bead chromatography or immunoisolation [147].

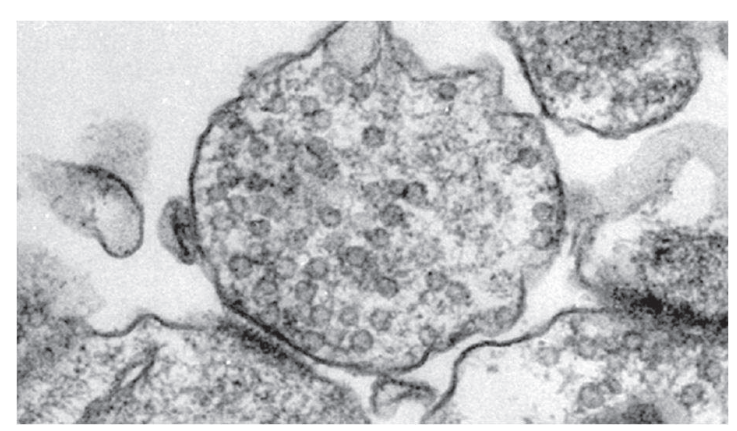

Figure 1.6: Image modidfied from [148]. An electron micrograph of a synaptosome prepared by shearing of brain tissue, showing pre- and postsynaptic compartments with retention of the adhesive contacts between the membranes at the synapse. 
Unlike these well established isolation protocols, it is technically difficult to obtain a presynaptic membrane preparation [149, 150]. Since proteomic analyses are strictly dependent on the availability of a purification protocol, studies on the presynaptic side have lagged behind. Although a first detergent based protocol to isolate a presynaptic fraction was reported in 2001 [151], the comprehensive proteomic analysis of this fraction 4 years later was not very clearcut with respect to both quantity and quality [152]. None of the active zone components were detected among the 110 identified proteins in this presynaptic fraction. Interestingly some of them were present in the analyzed PSD fraction. This was the only comprehensive proteomic study on the presynapse available by the time this project started. Only recently, another attempt to purify a presynaptic fraction by immunoisolation of docked vesicles revealed a larger number of proteins [153], but remained limited in comparison to the wealth of information that was obtained for the postsynaptic side.

\subsection{Aims of this Work}

Considering the observed electron density at the synapse and the fact that active zones determine not only the site and but also the timing for synaptic transmission, the number of known active zone proteins is surprisingly low. In comparison to the PSD, it is believed that the composition of presynaptic nerve terminal is only party uncovered yet. In order to fully understand the mechanisms that regulate the formation, maintenance and function of neurotransmitter release, it is necessary to reveal the exact protein composition of the active zone and analyze the interactions.

Due to the fact that proteomic studies mainly failed, because presynaptic preparations were scarce and insufficient, the main goal of this thesis was to develop an isolation protocol for a presynaptic fraction that allows for comprehensive proteomic studies. This protocol required an efficient removal of the postsynaptic density from synaptosomes, a challenging task that engaged a significant part of this work. Based on this protocol I wanted to validate presynaptic candidates and identify novel molecular players that are required for the docking of synaptic vesicles to the plasma membrane. By performing state-of-the-art quantitative proteomics, I hope to discriminate true presynaptic proteins from other contaminations. With this method I additionally wanted to describe changes in the presynaptic proteome in response to biological perturbations since this has not been done for the presynaptic site. Such an applicational example will hopefully provide a basis for further similar studies that will help to understand the mechanisms of synaptic transmission. 


\section{Material \& Methods}

\subsection{Materials}

\subsubsection{Chemicals}

Standard chemicals used in this study were obtained from either Sigma-Aldrich (Steinheim, Germany), Roth (Karlsruhe, Germany), Merck (Darmstadt, Germany), Boehringer (Ingelheim, Germany), Fluka (Buchs, Germany), Serva (Heidelberg, Germany), Roche (Basel, Switzerland) or Waters (Eschborn, Germany). All chemicals were of at least analytical purity. Other chemicals are listed below (2.1).

\begin{tabular}{|l|l|}
\hline Chemical & Source \\
\hline Pefabloc & Roche \\
Pepstatin & Peptide institute \\
Phenylmethylsulfonylfluorid (PMSF) & Roth \\
Eupergit C1Z beads & Roehm Pharma \\
GTP $\gamma$ S & Roche \\
GDP & Sigma Aldrich \\
Ni-NTA Agarose & Qiagen \\
5 ml MonoQ column & Amersham \\
RapiGest & Waters \\
Triethylammonium bicarbonate (TEAB) & Sigma Aldrich \\
Trifluoracetic acid & Sigma Aldrich \\
Formic acid & Fluka \\
Triton-X-100 & Sigma-Aldrich \\
Triton-X-114 & Sigma-Adrich \\
Dulbecco's Modified Eagle's Medium (DMEM) & Lonza \\
Penicillin/Streptomycin & Lonza \\
\hline
\end{tabular}

Table 2.1: Chemicals used in this study. 


\subsubsection{Enzymes}

The enzymes that were used in this study are listed in 2.2 and were obtained from Fermentas (St. Leon-Rot, Germany), New England Biolabs (NEB; Ipswich, MA, USA), Promega (Madison, WI, USA) or Roche (Basel, Switzerland). All restriction enzymes, ligases and polymerases were used according to manufacturer's instructions (including the supplied buffers).

\begin{tabular}{|l|l|l|}
\hline Enzyme & Application & Source \\
\hline Proteinase K & Synaptosome digest & Roche \\
Trypsin & Synaptosome digest & Roche \\
Trypsin (sequence grade modified) & In-solution digest for MS & Promega \\
Restriction enzymes & DNA digest & NEB or Fermentas \\
Ligase & DNA ligation & NEB \\
Pfu polymerase & Polymerase chain reaction & Promega \\
\hline
\end{tabular}

Table 2.2: Enzymes used in this study.

\subsubsection{Kits}

The commercially purchased kits used in this study are listed in 2.3 and were used for the stated application according to manufacturer's instructions (including the supplied buffers).

\begin{tabular}{|l|l|l|}
\hline Kit & Application & Source \\
\hline Western Lightening ${ }^{\mathrm{TM}}$ Plus-ECL & Chemoluminescence detection & Perkin Elmer \\
Pierce ${ }^{\circledR}$ BCA Protein assay & protein quantification & ThermoFisher \\
Lipofectamine ${ }^{\mathrm{TM}} 2000$ & transient cell transfection & Invitrogen \\
NucleoBond ${ }^{\circledR}$ Xtra & Plasmid purification (preparative scale) & Macherey-Nagel \\
NucleoSpin ${ }^{\circledR}$ Plasmid & Plasmid purification (analytical scale) & Macherey-Nagel \\
NucleoSpin ${ }^{\circledR}$ Extract & DNA clean-up & Macherey-Nagel \\
iTRAQ ${ }^{\text {TM }}$ reagent multiplex Kit & quantitative peptide labeling & Applied Biosystems \\
\hline
\end{tabular}

Table 2.3: Kits used in this study.

\subsubsection{Antibodies}

Antibodies used in this study are listed in Table 2.4. Antibodies were either generated in this laboratory or purchased at Abcam (Cambridge, UK), BD Bioscience (Erembodegem, Belgium), BioRad (Hercules,CA, USA), Jackson Immunoresearch Europe (Newmarket, UK), NeuroMab (Davis, USA), Synaptic Systems (Göttingen, Germany). 


\begin{tabular}{|c|c|c|c|c|}
\hline Antibody & Species & Epitope & Application & Source \\
\hline Synaptobrevin 69.1 & mouse monoclonal, ascites & SATAATVPPAAPAGEG & WB $(1: 2000)$ & [155] \\
\hline Munc13 & mouse, monoclonal, affinity purified & aa $3-317$ & WB $(1: 1000)$ & Synaptic Systems \\
\hline Piccolo & rabbit polyclonal, affinity purified & aa $439-4776$ & WB (1:500), IF (1:100) & Synaptic Systems \\
\hline Bassoon & rabbit polyclonal, serum & C-terminus & WB (1:500) & Synaptic Systems \\
\hline Synaptotagmin 41.1 & mouse monoclonal, ascites & cytoplasmic domain & WB (1:1000), IF (1:100) & [156] \\
\hline Syntaxin1A 78.2 & mouse monoclonal, ascites & $\mathrm{N}$-terminus & WB (1:1000), IF (1:100) & [46] \\
\hline NMDA receptor & mouse monoclonal, ascites & aa $660-811$ & WB $(1: 1000)$ & {$[157]$} \\
\hline AMPA receptor & rabbit polyclonal, affinity purified & C-terminus aa $826-906$ & WB (1:1000) & Synaptic Systems \\
\hline $\mathrm{Na}^{+} / \mathrm{K}^{+}$ATPase alpha 1 & mouse monoclonal, ascites & not known & WB (1:2000) & Abcam \\
\hline SDHA & mouse monoclonal, affinity purified & not known & WB (1:2000), IF (1:200) & Abcam \\
\hline Neuroligin & rabbit polyclonal, affinity purified & extracellular aa 46-165 & WB $(1: 1000)$ & Synaptic Systems \\
\hline $\mathrm{ERC} 1 \mathrm{~b} / 2$ & rabbit polyclonal, affinity purified & CDQDEEEGIWA & WB $(1: 1000)$ & Synaptic Systems \\
\hline GFP & rabbit polyclonal, serum & full length & WB $(1: 10000)$ & Synaptic Systems \\
\hline SynCAM & rabbit polyclonal, affinity purified & aa $167-181$ & WB $(1: 1000)$ & Synaptic Systems \\
\hline mouse $\operatorname{IgG}$ (Cy2 or Cy3 labeled) & goat polyclonal, affinity purified & $\operatorname{IgG}(\mathrm{H}+\mathrm{L})$ & $\operatorname{IF}(1: 400)$ & Jackson Immunoreserach \\
\hline rabbit IgG (Cy2 or Cy3 labeled) & goat polyclonal, affinity purified & $\operatorname{IgG}(\mathrm{H}+\mathrm{L})$ & $\operatorname{IF}(1: 400)$ & Jackson Immunoresearch \\
\hline mouse IgG (HRP labeled ) & goat polyclonal & $\operatorname{IgG}(\mathrm{H}+\mathrm{L})$ & WB $(1: 2000)$ & BioRad \\
\hline rabbit IgG (HRP labeled) & goat polyclonal & $\operatorname{IgG}(\mathrm{H}+\mathrm{L})$ & WB $(1: 2000)$ & BioRad \\
\hline
\end{tabular}

Table 2.4: Antibodies used in this study: IF (Immunfluorescence), WB (Western Blot), IP (Immunoprecipitation). Dilutions are marked in brackets. 


\subsubsection{Buffers and media}

\begin{tabular}{|c|c|}
\hline Buffer/media & Composition \\
\hline PBS & $\begin{array}{l}2.7 \mathrm{mM} \mathrm{KCl}, 1.5 \mathrm{mM} \mathrm{KH}{ }_{2} \mathrm{PO}_{4}, 137 \mathrm{mM} \mathrm{NaCl}, 8 \mathrm{mM} \\
\mathrm{Na}_{2} \mathrm{HPO}_{4}, \mathrm{pH} 7.3\end{array}$ \\
\hline TBST & 15 mM Tris-HCl, pH 7.4, 150 mM NaCl, 0.5 \% (v/v) Tween 20 \\
\hline SDS running buffer & $25 \mathrm{mM}$ Tris- $\mathrm{HCl}, 192 \mathrm{mM}$ Glycine, $0.1 \%$ SDS \\
\hline Transfer bu & $200 \mathrm{mM}$ Glycine, $25 \mathrm{mM}$ Tris, $0.04 \%$ SDS, $20 \%$ Methanol \\
\hline Hon & $320 \mathrm{~m}$ \\
\hline Sodium buffer & 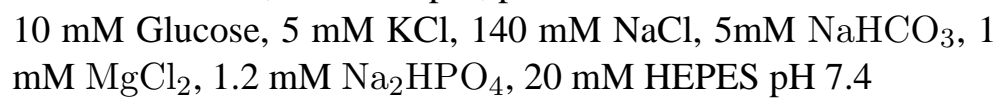 \\
\hline 1x IP buff & 1x PBS, 5 mM Hepes pH 8.0, 3 mg/ml BSA \\
\hline $2 \mathrm{x}$ IP & १M Hepes pH 8.0, 6 mg/ml BSA \\
\hline Cell culture media & $\begin{array}{l}\text { DMEM, } 10 \% \text { FCS, } 4 \mathrm{mM} \text { glutamine, } 100 \mathrm{U} / \mathrm{ml} \text { penicillin and } \\
\text { streptomycin }\end{array}$ \\
\hline Luria-Bertani medium (LB) & $10 \mathrm{~g}$ tryptone, $5 \mathrm{~g}$ yeast extract and $10 \mathrm{~g} \mathrm{NaCl}$ per $1 \mathrm{~L}$ \\
\hline
\end{tabular}

Table 2.5: Buffers and composition that were regularly used in this study.

\subsubsection{Mammalian cell lines and bacterial strains}

The Human Embryonic Kidney 293 cell line (HEK293) were used for over-expression studies. E.coli DH5 $\alpha$ strains were used for molecular cloning and E.coli BL21 (DE3) for protein expression.

\subsubsection{DNA constructs}

The plasmid encoding JB1 was synthesized and purchased from GENEART (Regensburg, Germany) according to the sequence obtained from NM_001108129. Codon usage was optimized for mammalian expression systems. The plasmid encoding GDP-dissociation inhibitor GDI (R. norvegicus) was a kind gift from Dr. Nathan Pavlos (University of Western Australia, Perth, Australia). 


\subsection{Methods}

\subsubsection{Molecular Biology Methods}

\subsubsection{Molecular Cloning}

The cDNA of JB1 was subcloned into the EGFP-N1 vector (Clontech) using XhoI and BamHI restriction sites for expression in HEK293 cells. GDI was subcloned into pET-28. Molecular cloning was performed with standard procedures for DNA restriction and purification, ligation of DNA constructs and transformation into competent DH5 $\alpha$-cells [159]. Plasmid purification was done according to the manufacturer's instructions (Macherey-Nagel). DNA primers were purchased from Sigma-Aldrich and DNA sequencing was done by MWG-Biotech AG.

\subsubsection{Protein Expression}

Recombinant His-tagged GDI was expressed and purified according to [160]. Briefly, GDIHis was expressed in E. coli-BL21(DE3) cells in $5 \mathrm{x} 500 \mathrm{ml} \mathrm{LB}$-medium at $37^{\circ} \mathrm{C}$. Protein expression was induced at $O D_{600}=0.6$ with $0.1 \mathrm{mM}$ IPTG. The bacteria were incubated for $14 \mathrm{~h}$ at $29^{\circ} \mathrm{C}$ and harvested by centrifugation at $4000 \mathrm{rpm}$ for $15 \mathrm{~min}$. Cell pellets were washed once with ice-cold PBS, frozen in liquid nitrogen and stored at $-80^{\circ} \mathrm{C}$. For purification, pellets were resuspended in $100 \mathrm{ml}$ cold lysis buffer (50 mM Tris, $1 \mathrm{mM}$ EDTA, $10 \mathrm{mM}$ $\beta$-mercaptoethanol, $\mathrm{pH} 8$ at $4{ }^{\circ} \mathrm{C}$ ), supplemented with $0.5 \mathrm{mg} / \mathrm{ml}$ lysozyme and incubated for 30 min at $4{ }^{\circ} \mathrm{C}$ followed by two freeze/thaw cycles (liquid $N_{2}, 32{ }^{\circ} \mathrm{C}$ ). $\mathrm{NaCl}$ was added to a final concentration of $300 \mathrm{mM}, \mathrm{MgCl}_{2}$ to $10 \mathrm{mM}$, sodium deoxycholate to $0.5 \mathrm{mg} / \mathrm{ml}$ and DNAse I to $0.05 \mathrm{mg} / \mathrm{ml}$. Samples were incubated for $45 \mathrm{~min}$ at $4{ }^{\circ} \mathrm{C}$ and centrifuged for $30 \mathrm{~min}$ at $135000 \mathrm{rpm}$. The GDI containing supernatant was combined with $5 \mathrm{ml} \mathrm{Ni-NTA-agarose}$ beads and rotated for $1 \mathrm{~h}$ at $4{ }^{\circ} \mathrm{C}$. The beads were collected in a column and washed with

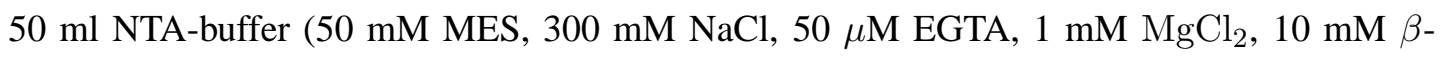
mercaptoethanol and $25 \mathrm{mM}$ imidazol, $\mathrm{pH}$ 6). GDI was eluted in $5 \mathrm{ml}$ steps with NTA-elution buffer (50 mM MES, $300 \mathrm{mM} \mathrm{NaCl}, 50 \mu \mathrm{M}$ EGTA, $1 \mathrm{mM} \mathrm{MgCl} 2,10 \mathrm{mM} \beta$-mercaptoethanol and $400 \mathrm{mM}$ imidazol, $\mathrm{pH}$ 6). Distribution of His-GDI was determined by SDS-PAGE and the fractions containing His-GDI dialyzed over night against 2 L MonoQ buffer ( $25 \mathrm{mM}$ Tris, $1 \mathrm{mM}$ DTT, $0.5 \mathrm{mM}$ EDTA, $1 \%$ sodium cholate, $\mathrm{pH} 7.4$ at $4{ }^{\circ} \mathrm{C}$ ). The dialyzed solution was filtered through a $0.22 \mu \mathrm{M}$ membrane and loaded on a $5 \mathrm{ml}$ MonoQ column (Amersham) using an Akta-purifier FPLC system (GE Healthcare). The protein was eluted with a linear gradient of 0-500 $\mathrm{mM} \mathrm{NaCl}$ in MonoQ buffer. Samples containing GDI were pooled, concentrated and dialyzed against 2x 11 25/125 buffer (25 mM HEPES-KOH, $125 \mathrm{mM}$ potassium acetate, $\mathrm{pH}$ 7.4). Proteins were frozen in liquid nitrogen and stored at $-80^{\circ} \mathrm{C}$. 


\subsubsection{Protein Determination}

Protein concentrations were determined using BCA [161]. Micro BCA assays were performed in a 96-well plate using Pierce BCA Protein Assay Kits (ThermoFischer) according to the manufacturer's manual.

\subsubsection{Cell Biological Methods}

\subsubsection{Cell Culture}

\section{HEK293}

Cells were cultured in the following growth medium (DMEM, 10\% fetal calf serum (FCS), 4 $\mathrm{mM}$ glutamine and 100 units $/ \mathrm{ml}$ each of penicillin and streptomycin). Cells were grown to $80 \%$ confluence on $10 \mathrm{~cm}$ culture dishes, at $37^{\circ} \mathrm{C}$ with $10 \% \mathrm{CO}_{2}$ and $90 \%$ humidity. HEK293 cells were passaged 3 times a week by detaching them from the plates using trypsin/EDTA (Lonza GmbH, Wuppertal, Germany).

\section{Primary Neurons}

High density hippocampal primary neurons were prepared from brains of newborn rats as describend in [162]. Neurons grown 10-14 DIV were used for the experiments performed here. The cultures were kindly provided by Martina Bremer (ENI, Goettingen).

\subsubsection{Transient Transfection}

HEK293 cells were seeded in 6-well plates one day before transfection. For transient transfection, the Lipofectamine 2000 transfection reagent was used. For each well, $4 \mu \mathrm{g}$ of purified plasmid DNA was mixed with $250 \mu \mathrm{l}$ DMEM without supplements. $10 \mu 1$ of Lipofectamine 2000 reagent were separately mixed with another $250 \mu \mathrm{l}$ DMEM (no supplements) and left for $5 \mathrm{~min}$ at room temperature. Afterwards, the lipofectamine-DMEM solution was mixed with the DNA-solution and left for 20-40 min at room temperature. Subsequently, a total volume of $500 \mu \mathrm{l}$ lipofectamine-DNA-mixture was added to the cells and incubated for 24 hours.

For expression level analysis of the transfected DNA, cells were lysed the next day. Therefore cells were washed once with ice-cold PBS and then incubated with $500 \mu$ lysis buffer (150 mM NaCl, 1 mM EDTA, 1\% Triton X-100, 50 mM HEPES-KOH, pH 7.3 supplemented with Complete protease inhibitor cocktail (Roche)) for $15 \mathrm{~min}$ on ice. The cell suspension was centrifuged for $10 \mathrm{~min}$ at $10000 \mathrm{rpm}$ at $4{ }^{\circ} \mathrm{C}$ and the supernatant analyzed by Western blotting. 


\subsubsection{Immunofluorescent Staining}

Hippocampal neurons were grown for 10-14 days in vitro (DIV) and fixed with 3.7\% paraformaldehyde (PFA) in PBS. PFA was removed and coverslips were washed 3 times with PBS for $5 \mathrm{~min}$ each. Afterwards, neurons were permeabilized by incubation with $0.3 \%$ Triton-X-100 in PBS for $5 \mathrm{~min}$ and washed again 3x 5min with PBS before they were blocked in 10\% normal goat serum (NGS) in PBS for $30 \mathrm{~min}$ at room temperature. Primary antibodies were diluted as indicated in $10 \%$ NGS/PBS. In a dark humidified chamber, coverslips were inverted on $25 \mu 1$ drops of antibody solution that were placed on parafilm. Incubation with primary antibodies was done for $1 \mathrm{~h}$. Afterwards, coverslips were replaced into the 24-well plate and washed for 3x 5 min with PBS. This procedure was repeated for incubation with the secondary antibody. Stained coverslips were mounted on microscope slides by inverting them on a drop of mounting medium (Fuoro-Gel, Electron Microscopy Sciences). Excess mounting medium was removed and samples solidified overnight at $4{ }^{\circ} \mathrm{C}$.

For immunofluorescent staining of synaptosomes, coverslips were pre-coated with poly-LLysine. $2 \mathrm{ml}$ synaptosomes collected from sucrose gradients (as described in section 2.2.4.1) were diluted in $5 \mathrm{ml}$ PBS and centrifuged for $30 \mathrm{~min}$ at $5500 \mathrm{~g}$ at $4{ }^{\circ} \mathrm{C}$. The synaptosomal pellet was resuspended in $2.4 \mathrm{ml}$ PBS. $200 \mu$ l of this synaptosomal solution was carefully placed on a coated coverslip placed in a 12-well plate and incubated for $45 \mathrm{~min}$ at room temperature. Afterwards, $1 \mathrm{ml}$ PBS was added to each well and synaptosomes pelleted on the coverslip by centrifugation for $30 \mathrm{~min}$ at $5500 \mathrm{rpm}$. Fixation and staining of synaptosomes was done as described for hippocampal neurons.

\subsubsection{Image acquisition and processing}

Neuronal and synaptosomal images were acquired using a AOBS SP2 confocal microscope (Leica Microsystems) with a 63x oil-immersion objective, standard filter sets (Leica Microsystems) and Leica LCS Lite software. For linescan analyses and overlays, images were processed using the LAS AF Lite software (Leica). 


\subsubsection{Biochemical Methods}

\subsubsection{SDS-PAGE and Western Blotting}

For the analysis of regular proteins $(<130 \mathrm{kDa})$, samples were separated in a $10 \%$ denaturating Tris/Tricine SDS polyacrylamide gel electrophoresis system, as described by [163] and [164]. The resolving gel (10\% bis-acrylamide, $0.1 \%$ SDS, $10 \%$ glycerol, $1 \mathrm{M}$ Tris $\mathrm{pH} 8.45$ ) and the stacking gel (4\% bis-acrylamide, $0.1 \%$ SDS, $1 \mathrm{M}$ Tris $\mathrm{pH} 8.45$ ) were polymerized by adding ammoniumpersulfate and TEMED . Samples were incubated $10 \mathrm{~min}$ at $70^{\circ} \mathrm{C}$ before loading. Separation was performed in a discontinuous buffer system, with a $0.2 \mathrm{M}$ Tris $\mathrm{pH} 8.9$ anode buffer and a $0.3 \mathrm{M}$ Tris $\mathrm{pH} 8.45,0.03 \%$ SDS cathode buffer. For the analysis of large proteins (>130 kDa), precasted NuPAGE ${ }^{\circledR}$ Bis-Tris gradient gels containing 4-12\% acrylamide were used. The NuPAGE ${ }^{\circledR}$ system is based upon a Bis-Tris- $\mathrm{HCl}$ buffered $\mathrm{pH} 6.4$ polyacrylamide gel, with a separating gel that operates at $\mathrm{pH}$ 7.0.

Western blotting was done according to [165]. Protein transfer from the gel to a nitrocellulose membrane was achieved in transfer buffer (200 mM Glycine, $25 \mathrm{mM}$ Tris, $0.04 \%$ SDS, $20 \%$ Methanol) by applying $50 \mathrm{~mA}$ for an hour using a semi-dry gel transfer apparatus. Large protein were transferred in MOPS buffer (Invitrogen) by using a tank apparatus and applying $40 \mathrm{~mA}$ for $1 \mathrm{~h}$. After transfer, membranes were blocked for $30 \mathrm{~min}$ at room temperature with blocking buffer (5\% nonfat milk powder in TBST) and then incubated with the primary antibody diluted in blocking buffer at $4{ }^{\circ} \mathrm{C}$ overnight. Membranes were washed 3 times with TBST for $10 \mathrm{~min}$ and then incubated with HRP-conjugated secondary antibodies in blocking buffer for 1 hour at room temperature. After another 3 washes (10 min each), membranes were covered with Western Lightening ${ }^{\mathrm{TM}}$ Plus-ECL and protein bands visualized by using chemiluminescence detection on a LumiImager (Boehringer Ingelheim).

\subsubsection{Protein Extraction with Triton-X-114}

Membrane proteins were enriched from cytosolic proteins using the detergent Triton-X-114 as described in [166]. Protein samples were diluted with PBS to a final concentration of 1 $\mathrm{mg} / \mathrm{ml}$. Triton-X-114 was added to a final concentration of $1 \%$ followed by an incubation on ice for $15 \mathrm{~min}$. Undissolved particles were removed by centrifugation for $5 \mathrm{~min}$ at $5000 \mathrm{rpm}$ and $4{ }^{\circ} \mathrm{C}$. To achieve phase partitioning, the supernatant was heated to $30{ }^{\circ} \mathrm{C}$ for $5 \mathrm{~min}$ and afterwards laid on top of a warm sucrose cushion consisting of $6 \%$ sucrose in PBS and $0.06 \%$ Triton-X-114. The sample was spun for $3 \mathrm{~min}$ at $300 \mathrm{~g}$ and room temperature in a swing-out rotor. The detergent phase containing hydrophobic proteins was collected as an oily droplet 
at the bottom of the tube. The extraction was repeated one more time and the final detergent pellet resuspended in ice cold PBS.

\subsubsection{Preparation of Immunobeads}

Conjugation of synaptophysin antibody to Eupergit C1Z beads to was done as described in [167]. Before the coupling procedure, synaptophysin antibody (ascites) was dialyzed extensively against $150 \mathrm{mM} \mathrm{NaCl}$ for 3 days with at least 7 changes. After dialysis, the solution was centrifuged for $15 \mathrm{~min}$ at $10000 \mathrm{~g}$ and the supernatant used for coupling. The desired amount of beads was washed twice with $\mathrm{H}_{2} \mathrm{O}$ by vortexing vigorously and applying ultrasonication in a waterbath for $2 \mathrm{~min}$. Beads were centrifuged for $6 \mathrm{~min}$ at $1300 \mathrm{~g}$ and resuspended in the synaptophysin solution containing at least $1 \mathrm{mg} / \mathrm{ml}$ antibody. $1 \mathrm{mg}$ antibody for $0.1 \mathrm{~g}$ beads was used. Beads were vortexed and rotated for $8 \mathrm{~h}$ at $21^{\circ} \mathrm{C}$. Afterwards, coupled beads were centrifuged for $6 \mathrm{~min}$ at $1300 \mathrm{~g}$ and the supernatant saved for protein determination in order to measure coupling efficiancy. $1 \mathrm{M}$ glycine was added to the bead pellet, resuspended by vortexing and rotated for at least $8 \mathrm{~h}$ at room temperature to quench remaining binding sites. Beads were washed 3 times alternating with $0.1 \mathrm{M}$ sodium acetate, $0.5 \mathrm{M} \mathrm{NaCl} \mathrm{pH} 4.5$ and $0.1 \mathrm{M}$ Tris, $0.5 \mathrm{M} \mathrm{NaCl} \mathrm{pH} 8.0$ (6 washes total). As a final step, beads were washed once with PBS and resuspended in 4 dry volumes PBS ( $4 \mathrm{ml}$ per $1 \mathrm{~g}$ beads). Beads can be stored at $-80^{\circ} \mathrm{C}$ without loss of activity.

\subsubsection{Preparation of Synaptosomes}

Synaptosomes were isolated from 6-weeks old wistar rats as described in [149]. Briefly, 2 rats were decapitated and cortices and cerebellum dissected. Samples were homogenized with a glass-teflon homogenizer in $30 \mathrm{ml}$ ice-cold homogenization buffer (320 mM sucrose, $5 \mathrm{mM}$ Hepes, pH 7.4) supplemented with PMSF/Pepstatin using 9 strokes at $9000 \mathrm{rpm}$. To remove cell debris, the homogenate was centrifuged $2 \mathrm{~min}$ at $5000 \mathrm{rpm}$ and $4{ }^{\circ} \mathrm{C}$ in a SS34 rotor. The supernatant was collected and re-centrifuged for $12 \mathrm{~min}$ at $11000 \mathrm{rpm}$. The supernatant containing brain cytosol was discarded and the synaptosome containing pellet resuspended in 5 $\mathrm{ml}$ homogenization buffer. A small brownish mitochondial fraction in the pellet was cautiously avoided. The suspension was laid on two 3-step discontinuous Ficoll gradients (one gradient per cortex) consisting of $4 \mathrm{ml} \mathrm{13 \%} \mathrm{Ficoll} \mathrm{(in} \mathrm{homogenization} \mathrm{buffer),} 1 \mathrm{ml} 9 \%$ Ficoll and $4 \mathrm{ml}$ 6\% Ficoll. Gradients were centrifuged $35 \mathrm{~min}$ in a SW41 swing-out rotor (Beckman) at 22500 rpm and the resulting band at interface between 13\% and 9\% Ficoll collected. Bands were diluted with $10 \mathrm{ml}$ homogenization buffer and pelleted by centrifugation for $12 \mathrm{~min}$ at 11000 rpm. The final synaptosomal pellet was resupended in $5 \mathrm{ml}$ fresh homogenization buffer and 
the protein concentration was determined using BCA. Generally, yields range between 7-10 mg synaptosomes per 2 rat brains.

\subsubsection{Protease Treatment of Synaptosomes}

Five mg synaptosomes (isolated as described above) were carefully centrifuged for $3 \mathrm{~min}$ at $8700 \mathrm{~g}$. The pellet was resuspended in $20 \mathrm{ml}$ sucrose buffer $(320 \mathrm{mM}$ sucrose, $5 \mathrm{mM}$ Hepes, $\mathrm{pH} 8$ at room temperature) and supplemented with $500 \mu \mathrm{l}$ of a trypsin solution $(0.1 \mathrm{mg} / \mathrm{ml}$, Roche) resulting in a protein-protease ratio of 100:1. Synaptosomes were incubated for 30 min at $30^{\circ} \mathrm{C}$ with occasional mixing. Afterwards, synaptosomes were pelleted again for $3 \mathrm{~min}$ at $8700 \mathrm{~g}$ and protease activity was stopped by resuspending the pellet in the desired amount sucrose buffer containing $400 \mu \mathrm{M}$ Pefabloc.

\subsubsection{Separation of Protease Treated Synaptosomes from the PSD}

Protease treated synaptosomes (as described above) were resuspended in $5 \mathrm{ml}$ sucrose buffer containing $400 \mu \mathrm{M}$ Pefabloc. To separate shaved synaptosomes from the postsynaptic densities, $3 \mathrm{ml}$ of the sample were loaded on a continuous sucrose gradient $(25-50 \%(\mathrm{w} / \mathrm{v})$ sucrose in $5 \mathrm{mM}$ Hepes $\mathrm{pH}$ 8.0) and centrifuged for $3 \mathrm{~h}$ at $180000 \mathrm{~g}(28000 \mathrm{rpm})$ in a SW28 swing-out rotor (Beckman). Continuous sucrose gradients were generated with an automatic gradient mixer (Gradient master, Biocomp) aacording to the manufacturer's instructions. After centrifugation, $1.5 \mathrm{ml}$ fractions were collected from the gradient from bottom to top using a semi-automatic pump system (Minipuls3, Abimed Gilson).

Fractions containing digested synaptosomes, so called "shaved" synaptosomes, were either identified by measuring the refraction index or dot blotting. Shaved synaptosomes were found in the fractions with a refraction index of 1.391-1.392, which corresponds to 1.2 M sucrose. For dot blotting, $2 \mu \mathrm{l}$ of each fraction was spotted on a dry nitrocellulose membrane and soaked in for $5 \mathrm{~min}$. Afterwards, the membrane was incubated for $10 \mathrm{~min}$ at room temperature in blocking buffer (5\% non-fat milk powder in TBST). Incubation with the primary antibody against synaptophysin was done for 15 min in blocking buffer. After washing the membrane 3 times for 3 min each with blocking buffer, the blot was incubated with secondary antibody for $15 \mathrm{~min}$. After another 3 washes ( 3 min each) with TBST, membranes were incubated with

Western Lightening ${ }^{\mathrm{TM}}$ Plus-ECL and protein bands visualized by using chemiluminescence detection on a LumiImager. 


\subsubsection{Immunoisolation of Docked Vesicles from Protease treated Synaptosomes}

Protease-treated synaptosomes (as described previously) were resuspended in $300 \mu \mathrm{l}$ sucrose buffer containing $400 \mu \mathrm{M}$ Pefabloc. Synaptosomes were lysed by adding $2.7 \mathrm{ml}$ ice-cold $\mathrm{H}_{2} \mathrm{O}$ followed by homogenization with a glass-teflon homogenizer with 3 strokes at maximum speed. Afterwards, $15 \mu \mathrm{l} 1 \mathrm{M}$ Hepes pH 8, $3 \mu \mathrm{l} 200 \mathrm{mM}$ PMSF (in ethanol) and $3 \mu \mathrm{l} 2$ $\mathrm{mg} / \mathrm{ml}$ pepstatin (in DMSO) were added immediately to the solution. Docked and free synaptic vesicles were separated on a $15-45 \%$ continuous sucrose gradient $(w / v)$ prepared with a gradient mixer (Gradient master, Biocomp). Samples were loaded on the gradient and centrifuged for $1 \mathrm{~h}$ at $100000 \mathrm{~g}$ in a SW28 swing-out rotor (Beckman). Twenty-four $1.5 \mathrm{ml}$ fractions were collected from bottom to top and analyzed by dot blotting for synaptophysin (as described before). Docked vesicles were generally localized in fractions 4-7 and free vesicles in fractions 19-21. Fractions containing docked vesicles were pooled (SPM), the same was done for fractions containing free vesicles (SV). For one immunoisolation, $5 \mu \mathrm{l}$ Eupergit $\mathrm{C} 1 \mathrm{Z}$ beads coupled to the antibody for synaptophysin (Eupergit-7.2) were washed with 1x IP buffer (1x PBS, $3 \mathrm{mg} / \mathrm{ml} \mathrm{BSA,} 5 \mathrm{mM}$ Hepes $\mathrm{pH}$ 8.0). For docked vesicles, $600 \mu \mathrm{l} \mathrm{SPM}$ fraction and 600 $\mu 12 x$ IP buffer (2x PBS, $6 \mathrm{mg} / \mathrm{ml}$ BSA, $5 \mathrm{mM}$ Hepes $\mathrm{pH}$ 8.0) were added to the bead pellet. For the isolation of free vesicles, $300 \mu \mathrm{l} \mathrm{SV}$ fraction and $900 \mu \mathrm{l} 1 \mathrm{x}$ IP buffer was used. Beads were gently vortexed and rotated over night at $4{ }^{\circ} \mathrm{C}$. Afterwards, beads were spun down for 3 $\min$ at $2000 \mathrm{rpm}$ in a table top centrifuge. Immunoisolates were washed 3 times with PBS by vortexing, incubation on ice for $5 \mathrm{~min}$ and centrifugation for $3 \mathrm{~min}$ at $2000 \mathrm{rpm}$. After a final centrifugation step for $3 \mathrm{~min}$ at $10000 \mathrm{~g}$, immunoisolates were either eluted by adding 2x LDS sample buffer and incubation for $10 \mathrm{~min}$ at $70^{\circ} \mathrm{C}$ or were processed for mass spectrometric analysis according to the iTRAQ labeling method (see section 2.2.5.1

\subsubsection{Mass Spectrometry Methods}

\subsubsection{1 iTRAQ labeling}

Ten immunoisolations for both docked and free vesicles were done simultaneously and samples from 5 immunoisolations were pooled after the washing steps, resulting in 4 final samples ( $2 \mathrm{x}$ SPM, 2x SV). Immunoisolated docked and free SVs were then solubilized by resuspension of the bead pellets in $28 \mu \mathrm{l} 1 \%$ RapiGest SF (Waters) in $100 \mathrm{mM}$ triethylammonium bicarbonate (TEAB) buffer and incubated for $10 \mathrm{~min}$ at $70^{\circ} \mathrm{C}$. Solubilized proteins were digested in the presence of the beads by trypsin according to [168]. Briefly, $4 \mu 1100 \mathrm{mM}$ TEAB buffer and 2 $\mu \mathrm{l}$ reducing agent (supplied with the iTRAQ Kit) were added to the beads and incubated for $1 \mathrm{~h}$ at $37^{\circ} \mathrm{C}$ with shaking $(750 \mathrm{rpm})$. Afterwards, the bead suspension was supplemented with $1 \mu \mathrm{l}$ 
$200 \mathrm{mM}$ iodoacetamide and incubated for another $20 \mathrm{~min}$ at $37^{\circ} \mathrm{C}$ with shaking $(750 \mathrm{rpm})$. For digestion of the proteins, $5 \mu \mathrm{l}$ trypsin $(0.2 \mu \mathrm{g} / \mu \mathrm{l}$ in $100 \mathrm{mM}$ TEAB, Promega) was added and incubated overnight at $37^{\circ} \mathrm{C}$ with shaking. Beads were pelleted for $20 \mathrm{~min}\left(4^{\circ} \mathrm{C}\right)$ at maximum speed in a table top centrifuge and the supernatants transferred to clean tubes. Tryptic peptides were then tagged with iTRAQ reagent according to manufactures instructions (iTRAQ reagent kit, Applied Biosystems). iTRAQ reagents were spun down before use and ethanol added to a final volume of $170 \mu \mathrm{l}$ per tube. Eighty-five $\mu \mathrm{l}$ of reagent was added to both of each sample (docked SVs were tagged with iTRAQ 117, and free SVs with iTRAQ 116) and incubated for $3 \mathrm{~h}$ at $37^{\circ} \mathrm{C}$ with shaking $(750 \mathrm{rpm})$. Afterwards, each sample labeled with iTRAQ-116 was mixed with one labeled with iTRAQ-117. The resulting samples were supplemented with 20 $\mu 15 \%$ trifluoracetic acid (TFA) for $\mathrm{pH}$ adjustments $(\mathrm{pH}=2)$ and incubated for $1 \mathrm{~h}$ at $37^{\circ} \mathrm{C}$ with shaking. After centrifugation for $30 \mathrm{~min}$ at maximum speed at $4{ }^{\circ} \mathrm{C}$, supernatants were of all samples were combined in one tube.

\subsubsection{SCX Fractionation}

After tryptic diegestion and iTRAQ labeling the peptides were fractionated manually over an ICAT strong cation-exchange (SCX) column (Applied Biosystems) according to the manufacturer's instructions. The sample volume of about $800 \mu \mathrm{l}$ was reduced to less than $200 \mu \mathrm{l}$ using a vacuum centrifuge at medium heat and then diluted in $2 \mathrm{ml} \mathrm{SCX}$ loading buffer $(10 \mathrm{mM}$ $\mathrm{KH}_{2} \mathrm{PO}_{4}, 25 \%$ acetonitrile, $\mathrm{pH}$ 3.0). The SCX column was equilibrated by injecting $2 \mathrm{ml}$ of loading buffer before the sample was slowly loaded. The column was washed by injecting 1 $\mathrm{ml}$ of loading buffer. Peptides were eluted stepwise by adding $500 \mu \mathrm{l}$ of $\mathrm{KCl}$-solutions of increasing concentration $(5,100,150,200,300,400,500,600,800$, and $1000 \mathrm{mM})$ in $10 \mathrm{mM}$ $\mathrm{KH}_{2} \mathrm{PO}_{4}, 25 \%$ acetonitrile, $\mathrm{pH}$ 3.0. The samples were desalted on a hand made micro column with POROS Oligo R2 RP material as described in [169]. Briefly, all 10 SCX fractions were dried in a vacuum centrifuge at medium heat until salt precipitation has occurred and the precipiates were resuspended in $100 \mu \mathrm{l} 0.3 \%$ TFA. High salt fractions (800 and $1000 \mathrm{mM} \mathrm{KCl}$ ) were resuspended in $200 \mu \mathrm{l}$ of $0.3 \%$ TFA. Samples were incubated for $30 \mathrm{~min}$ at room temperature with shaking. Each SCX fraction was cleaned as followed: The handmade RP column was washed by applying $100 \mu \mathrm{l}$ of $0.1 \%$ TFA with a syringe, followed by loading of the sample on the column, a washing step with $100 \mu \mathrm{l}$ of $0.1 \%$ TFA and an elution by applying $20 \mu \mathrm{l}$ of

$50 \%$ acetonitrile, $0.5 \%$ formic acid. At the end all remaining peptides were eluted with 20 of $\mu 190 \%$ acetonitrile. Samples were dried using a vacuum centrifuge and stored at $-20^{\circ} \mathrm{C}$. 


\subsubsection{Mass Spectrometry and Quantification}

The SCX-fractions were dissolved in $12 \mu \mathrm{l} 5 \%$ formic acid of which $5 \mu \mathrm{l}$ were analyzed on a Thermo LTQ XL Orbitrap (Thermo Fisher Scientific) that is coupled to an Agilent 1100 series LC-system (Agilent Technologies). In the LC system, peptides were separated at a flow rate of 200-300 nl/min on a self-made reversed phase column (C18, Reprosil, Maisch) and eluted with a 118 min gradient from $7.5-40 \%$ mobile phase B ( $80 \%$ acetonitrile, $0.15 \%$ formic acid). Peak list analysis was done by searching against NCBI RefSeq database of r.norvegicus using Mascot v.2.2.04 as the search engine. Mass accuracy was $10 \mathrm{ppm}$ for the parent ion and 30 ppm for fragment ions. For the analysis, only tryptic peptides with a maximum of 2 missed cleavages were taken into account. Fixed modifications included carbamidomethylation of cysteines, whereas oxidations of methionine residues were considered as variable modification. Quantification was done by using Mascot v 2.2.04. and was constrained to peptides with a scores $>15$. The protein ratio was calculated as a weighted median ratio, but only included proteins quantified with unique peptides and a minimum of 3 peptides.

\subsubsection{Data Normalization}

The analysis of the statistical distribution of the docked/free vesicles peptide and protein ratios from each biological replicate showed a minor bias towards the free vesicles in one biological replicate, indicating that the number of immunoisolated vesicles was slightly more than in the docked vesicle sample in this replicate. A normalization to equal amounts of vesicles was done on the protein level, taking the vacuolar ATPase as a reference. For this replicate, all protein ratios were multiplied by 0.67 resulting in a balanced distribution around a 1.1 ratio for all major SV proteins. In the other two biological replicates, the amount of immunoisolated vesicle proteins within the docked and free vesicle fractions were comparable without normalization.

\subsubsection{Rab Extraction Assay}

Rab extraction by GDI was performed as described in [170]. Docked vesicles were collected from the sucrose gradient (as described in section 2.2.4.2) and diluted 1:1 in 2x IP buffer ( $2 \mathrm{x}$ PBS, $5 \mathrm{mM}$ Hepes $\mathrm{pH}$ 8.0, $6 \mathrm{mg} / \mathrm{ml}$ BSA). Samples were supplemented with GDP or GTP $\gamma \mathrm{S}$ to a final concentration of $500 \mu \mathrm{M}$ and Complete protease inhibitor cocktail (Roche) and kept on ice for $15 \mathrm{~min}$. Afterwards purified GDI (see 2.2.1.2) was added to a concentration of 0.5 $\mu \mathrm{M}$ and samples incubated for $30 \mathrm{~min}$ at $37^{\circ} \mathrm{C}$.

Samples were either further processed by immunoisolation of vesicles (as described in section 2.2.4.2) or fractionated by a floatation assay. For the floatation assay, a 3-step discon- 
tinuous sucrose gradient was generated by overlaying $1 \mathrm{ml}$ of the sample with $500 \mu 10.7 \mathrm{M}$ sucrose and $500 \mu \mathrm{l} 0.32 \mathrm{M}$ sucrose. The gradient was centrifuged for $3 \mathrm{~h}$ at $255000 \mathrm{~g}$ in a TLS-55 rotor (Beckman) and fractions carefully collected from the top by pipetting $200 \mu \mathrm{l}$ aliquots.

\subsubsection{Electron Microscopy}

Electron microscopy on synaptosomes was carried out by Dr. Dietmar Riedel (MPI for Biophysical Chemistry, Goettingen). Synaptosomes were fixed by aldehydes and embedded in epon for preparing ultra-thin sections. Sections were analyzed using a Philips $120 \mathrm{kV}$ BioTwin microscopes equipped with a $1024 \times 1024$ pixel GATAN CCD camera. 


\title{
3 Results
}

\subsection{Establishment of a protocol for the isolation of presynaptic membrane fractions}

\begin{abstract}
Although many key players of the presynaptic active zone have been identified, the exact molecular architecture of the sites at which synaptic vesicles are attached to the plasma membrane is still not fully understood. To provide insights into the protein composition of synaptic vesicle docking sites, it is necessary to isolate such fractions with sufficient purity. So far it has been very challenging to separate presynaptic membranes and the postsynaptic protein scaffold. The protocol developed in this work is based on the biochemical properties of conventionally isolated synaptosomes [149]. Synaptosomes are resealed nerve terminals. As such they are membrane enclosed sacks that contain the presynaptic excitatory machinery as well as cytosolic proteins and other organelles e.g. mitochondria. Additionally, a portion of the postsynaptic density remains tightly attached on the outside of these resealed compartments and accounts for its copurification with the presynaptic compartment [171]. With this in mind, the approach employed here includes a mild proteolysis of the synaptosomes to remove the postsynaptic membranes. Such a proteolytic treatment is expected to cleave the synaptic adhesion molecules responsible for the tight attachment between pre- and postsynaptic components as well as the extracellular domains of receptors and ion channels. In contrast, components of active zones are protected from proteolytic degradation since the membrane enclosing the presynaptic compartment presents a physical barrier against protease entry. Unfortunately, most of the PSD proteins appeared to be largely resistant to proteolysis. Therefore, an additional sucrose gradient centrifugation step was necessary to effectively separate PSD components from presynaptic proteins according to their mass and density (see Fig 3.1 A). As mentioned above, the interior of synaptosomes include mitochondria, docked as well as free vesicles and cytosolic proteins. To separate these contents, the protease treated and purified synaptosome fraction was lysed by osmotic shock prior to the continuous sucrose density gradient centrifugation. In a final step, docked vesicles and free vesicles were purified from a fraction of this gradient by
\end{abstract}


immunoisolation using Eupergit $\mathrm{C} 1 \mathrm{Z}$ beads containing immobilized antibodies specific for the synaptic vesicle protein synaptophysin (Fig. 3.1B).

\subsubsection{Removal of the postsynaptic density from synaptosomes}

\subsubsection{Optimization of the protease treatment and separation of pre- and postsynaptic compartments}

Following the purification of synaptosomes according to the protocol described by [149], the synaptosomes were resuspended in a suitable buffer and several modifications to a basic digestion protocol were systematically introduced and experimentally assessed for their effectiveness in removing PSD components from intact synaptosomes. The basic digestion protocol involves the following steps:

(a) Digestion of synaptosomes with proteases

(b) Stopping protease activity

(c) Harvesting of the digested synaptosome

Modifications to this protocol are described in the remainder of this section. Of the commercially available proteases, trypsin and proteinase $\mathrm{K}$ were tested in this study for their efficiancy in removing the PSD. Trypsin is the preferred protease, because it is compatible with subsequent sample preparation procedures for mass spectromic analysis. Proteinase K with its versatility and consistent activity in a broad range of conditions was tested as an alternative candidate. The enzymatic activity of proteases depends on several parameters including their concentration (protein:protease ratio), incubation temperature and time, buffer composition. To obtain the optimal digestion conditions, each of these parameters were systematically tested.

The buffer system was first chosen to provide basic conditions for optimal digestion. Two different buffer systems are commonly used when working with synaptosomes. A low ionic strength sucrose buffer ( $320 \mathrm{mM}$ sucrose, $5 \mathrm{mM}$ HEPES $\mathrm{pH}$ 7.4) is used during the preparation of synaptosomes and a moderate salt solution "sodium buffer" (10 mM Glucose, $5 \mathrm{mM}$

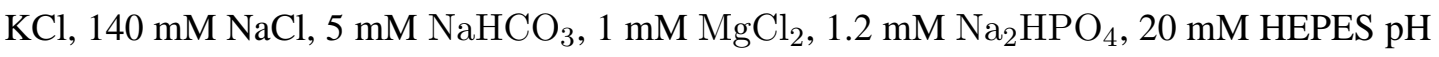
7.4) is often employed in stimulation-dependent glutamate release assays [149]. Both buffer systems were tested for their compability with the selected proteases. Trypsin was added to the synaptosomes at a protein/protease ratio of 1:300, followed by an incubation for 10-60 


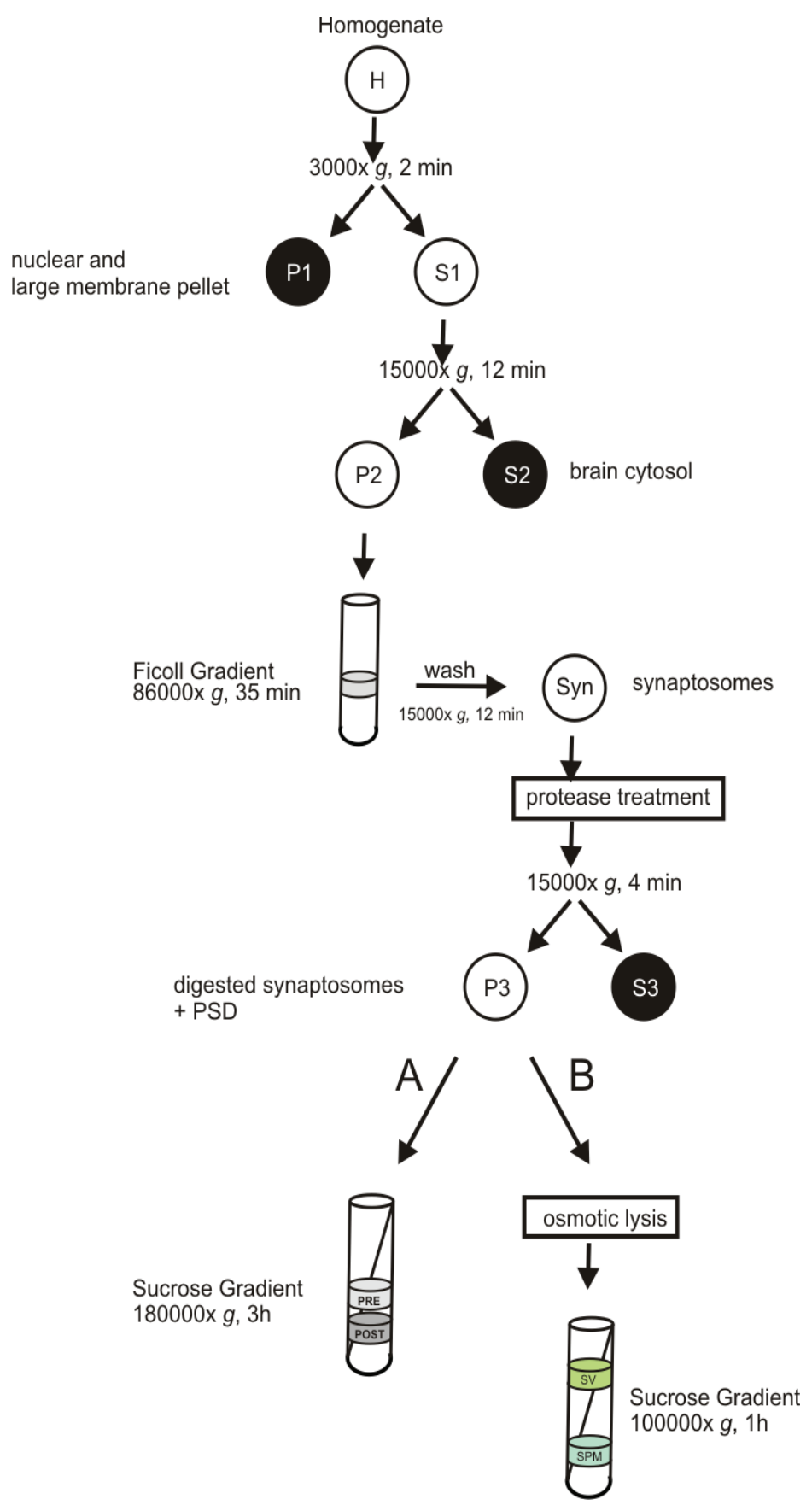

Figure 3.1: Schematic overview of the protocol for isolating presynaptic membrane fractions. Synaptosomes were prepared as described in [149] and subjected to mild protease treatment to digest the proteins in the synaptic cleft. Afterwards, shaved synaptosomes were either (A) separated from postsynaptic membranes in a continuous sucrose gradient afterwards or (B) lysed and fractionated on a continuous sucrose gradient yielding a docked vesicle fraction (SPM) and and a free vesicle fraction (SV). 
minutes at $30^{\circ} \mathrm{C}$. Afterwards the enzyme activity was stopped by adding the irreversible serine protease inhibitor Pefabloc to the sample. Synaptosomes were then pelleted and washed once with buffer. The efficiency of digestion was assayed by monitoring the amount of postsynaptic receptor NR1 remaining after the reaction was stopped. Additionally, levels of the presynaptic protein Munc18 and the synaptic vesicle protein synaptophysin were also analyzed to determine if undesired proteolytic degradation had occured in the presynaptic compartments. Interestingly, synaptosomal digestion by trypsin in sucrose but not sodium buffer led to a gradual decrease in the amount of NR1 (Fig 3.2 A). In both buffers, proteins localized inside the synaptosome are not affected. This result indicated that the sodium buffer is unsuitable for use as a buffer in this procedure. One likely explanation for this is that synaptosomes appear to aggregate in the sodium buffer but not the sucrose buffer after the pelleting steps in the procedure.

Next, the efficiancy of trypsin versus proteinase K was assessed. As shown in Fig. 3.2 B, proteinase $\mathrm{K}$ is a more potent protease that also digested proteins inside the synaptosome exemplified by Munc18. In theory, this protein is protected by the synaptosomal membrane and should not be degraded. Nevertheless, these experiments indicated that proteinase K was able to enter the synaptosome. Interestingly, proteins residing in the vesicle membrane were not affected by any of the proteases. Since proteinase K digested proteins inside of the synaptosomal membrane, trypsin was used in all further experiments.

The effect of temperature and time on the effectiveness of trypsin digestion was subsequently examined. As expected, proteolytic activity increases at higher temperature (Fig 3.2 C). However, for the purification of the docked vesicle fraction it was deemed important to keep the temperature as low as possible. Elevated temperatures raise the possibility of unwanted endogenous protein degradation within the synaptosome as well as other temperature-dependent effects on biological activity (e.g. exocytosis). Consequently, although initial experiments were performed at $30{ }^{\circ} \mathrm{C}$, digestions at $25^{\circ} \mathrm{C}$ was also tested (Fig. $3.2 \mathrm{C}$ ). At $25^{\circ} \mathrm{C}$, NR1 was inefficiently cleaved, suggesting this temperature is insufficient for optimal proteolytic activity despite prolonging the extension time to $60 \mathrm{~min}$. For practical issues, a further extension of the incubation time longer than 60 minutes was not considered.

Finally, the concentration of trypsin was optimized. Protease/protein ratios of 1:100, 1:200, 1:300 and 1:500 were tested and the efficiency of digestion evaluated over different time intervals. As shown in Fig. 3.2 D, cleavage of NR1 is significantly enhanced at higher protease:protein ratios. At a ratio of 1:100, NR1 became undectable after a 20 minute digestion. In contrast, complete abolishment of the signal was not achieved even after 60 minutes when a 1:500 protease/protein ratio was used. Importantly, the stability of the presynaptic proteins 

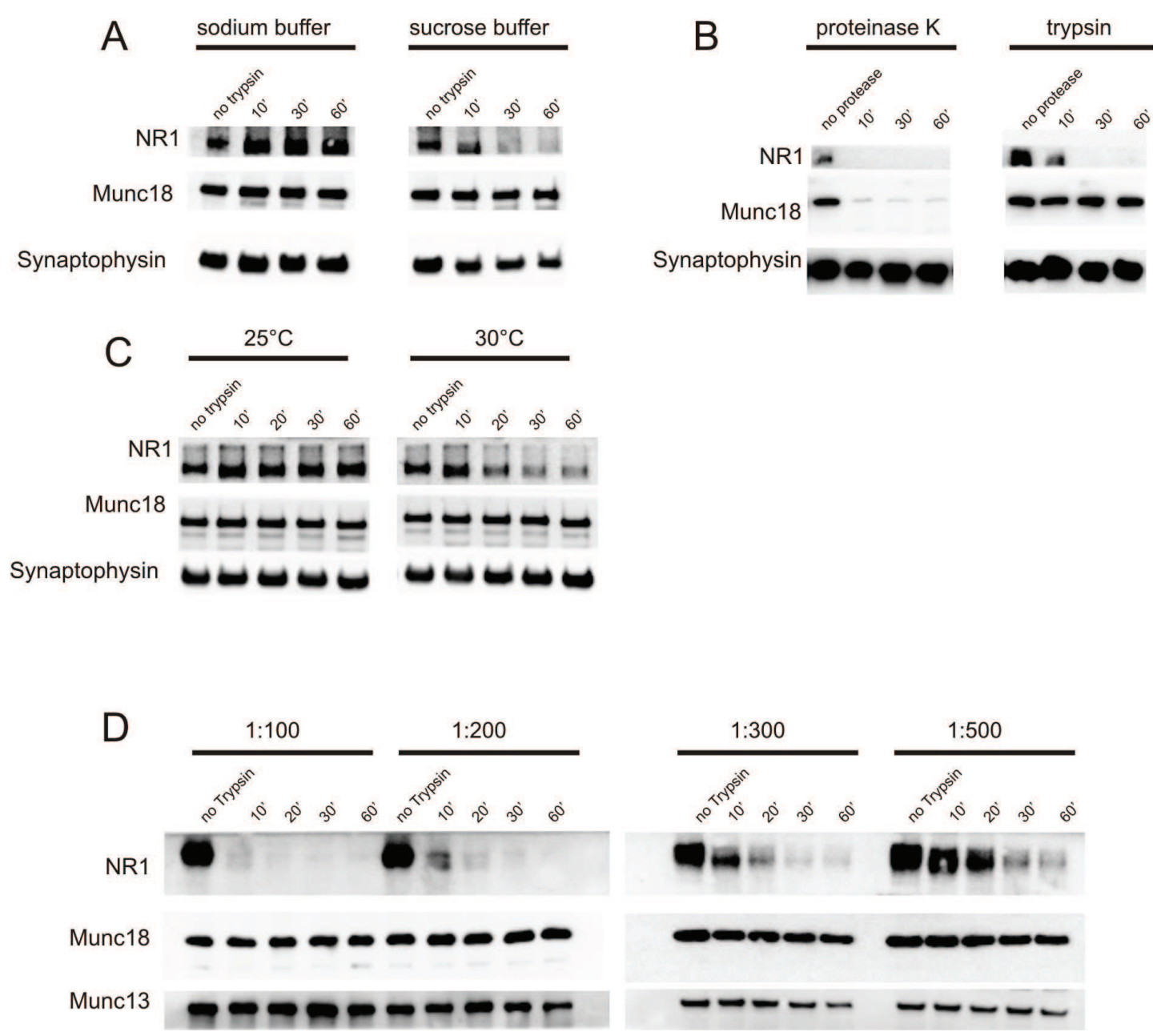

Figure 3.2: Optimization of proteolysis. (A) Proteolytic efficiency in different buffer systems. By using trypsin (protease:protein ratio of 1:300), the postsynaptic NR1 receptor was time-dependent digested in sucrose buffer but not in sodium buffer. Proteins inside the synaptosomal membrane (Munc18 and synaptophysin) are not digested. (B) Comparison of synaptosomal digestion by trypsin and proteinase K. Trypsin induced degradation only affected proteins outside the synaptosomal membrane (NR1). Proteinase K dependent digestion was additionally observed for cytosolic proteins inside of the synaptosome, e.g. Munc18. (C) Temperature dependency of proteolytic activity. NR1 digestion (protease:protein ratio of 1:300) was observed after $20 \mathrm{~min}$ at $30^{\circ} \mathrm{C}$, but was not achieved after 60 $\min$ at $25^{\circ} \mathrm{C}$. (D) Effect of trypsin concentration and incubation time on the cleavage of postsynaptic membranes. Digestion of NR1 was time- and concentration-dependant. For NR1 cleavage, a protease:protein ratio of 1:100 was sufficient after $10 \mathrm{~min}$ whereas the same degree of digestion needed $60 \mathrm{~min}$ at a 1:500 ratio. Presynaptic proteins (Munc13 and Munc18) remain unaffected. 
Munc18 and Munc13 remains unaffected. Based on these data, the final digestion protocol was performed using a 1:100 ratio of trypsin to protein concentration in sucrose buffer $(320 \mathrm{mM}$ Hepes, $\mathrm{pH}$ 8) with incubation time of 30 minutes at $30^{\circ} \mathrm{C}$.

To better characterize the effect of protease treatment on synaptosomes, additional synaptic proteins were analyzed for their stability by western blotting after trypsinization (see Fig. 3.3). As expected, adhesion molecules that span the synaptic cleft were efficiently degraded after the 30 min digestion. In contrast, presynaptic proteins (including large proteins like Piccolo or Munc13) and synaptic vesicle proteins remain intact. The postsynaptic receptors NR1 and GluR1 appear to be digested. In fact, the epitopes of these proteins recognized by the antibodies used are mapped to the extracellular domains facing the synaptic cleft. Surprisingly, the postsynaptic proteins PSD95 and Homer were resistant to proteolysis. Conceivably, the localization of these proteins deep within the protein network of the postsynaptic specialization renders them less accessible to proteases.

Thus, while the protease treatment succeeded in removing trans-synaptic molecules, certain integral components of the PSD persisted. This suggested that the tight attachment of the PSD to the synaptosome is abolished and led to the idea to separate pre- and postsynaptic compartment according to their biochemical nature. This idea was tested by adding a sucrose density gradient step after the digestion protocol.

\subsubsection{Separation of pre- and postsynaptic compartments}

It is well known that pre- and postsynaptic specializations differ with respect to ultrastructure and biochemical properties [126]. PSDs appear proteinaceous compared to the presynaptic boutons, which are more membraneous. Thus, buoyant densities are suggested be different. In fact, early experiments showed that purified postsynaptic densities are enriched at a 1.4/2.2 M sucrose interface of a discontinuous sucrose gradient [172] whereas synaptosomes are localized to 1.0/1.2 $\mathrm{M}$ interfaces in sucrose gradients [150]. Hypothesizing that there might be a shift in the density of the shaved membranous synaptosome versus the protein dense PSD after digestion, a $0.75 \mathrm{M}-1.5 \mathrm{M}$ continuous sucrose gradient was chosen to attempt separation of the postsynaptic density from the shaved synaptosome. Indeed, a shift in the migration of PSD95 was observed in protease treated synaptosomes that was not seen for untreated samples (see Fig. 3.4A). Importantly, the signals of the pre- and postsynaptic markers no longer showed

significant overlap, indicating that the remaining synaptosomes were devoid of the postsynaptic density. These "shaved" synaptosomes sediment at fractions with an refraction index of 1.391, which corresponds to about $1.2 \mathrm{M}$ sucrose. Additionally, electron microscopy was used to assess if the protease treatment affected the integrity of or induced ultrastructural changes to 


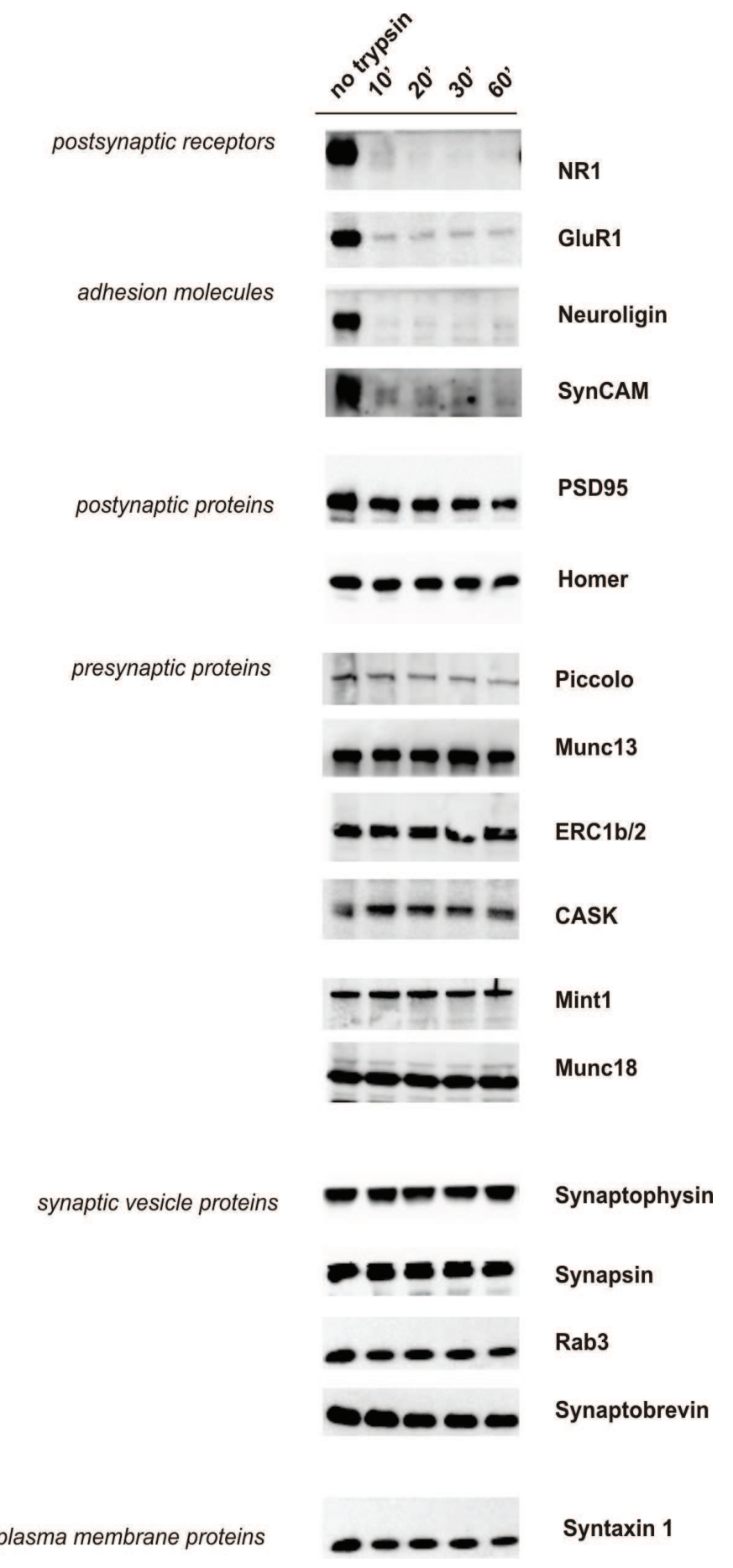

Figure 3.3: Effect of protease treatment on various protein groups. Presynaptic proteins and synaptic vesicle proteins are not affected in their stability after digestion whereas postsynaptic receptors and adhesion molecules are efficiently cleaved. Proteins localized deep inside the postsynaptic density appear resistent to proteolysis. 
the synaptosomes. As shown in Fig. 3.4 B, the morphology of synaptosomes was unchanged after trypsination. Specifically, the synaptosomal plasma membrane was intact and the interior filled with synaptic vesicles. Lower magnification electron microscopy pictures also showed that the quantity of synaptosomes appear in the same range.

To validate that the second centrifugation step indeed resolves the two compartments, immunofluorescence microscopy was employed to analyze the distribution of pre- and postsynaptic marker proteins in the protease treated synaptosomes after sucrose gradient centrifugation. Therefore, synaptosomal fractions corresponding to $1.2 \mathrm{M}$ sucrose were collected from the gradient by pelleting on poly-L-lysine coated coverslips and analyzed. The samples were then immunostained with antibodies directed against synaptophysin and PSD95 and their distribution examined by confocal microscopy. Trypsination of synaptosomes is expected to change the intensity of the fluorescence signal detected for postsynaptic proteins (proportional to the protein amount) and extent of co-localization between PSD95 and synaptophysin. As shown in Fig. 3.5 A, untreated synaptosomes displayed a very high degree of co-localization of both proteins. In contrast, shaved synaptosomes exhibited both a decrease in PSD95 puncta as well as an abolishment of co-localization between the two proteins. No changes in the signal intensities for synaptophysin was observed. Linescans analysis of the images further verified these observations (Fig $3.5 \mathrm{~B}$ ). Thus, the data demonstrate that the established protocol efficiently removes postsynaptic densities from synaptosomes.

\subsubsection{Isolation of a fraction enriched in docked vesicles}

\subsubsection{Lysis of synaptosomes}

It is well established that synaptosomes undergo lysis and release synaptic vesicles and mitochondria when they are exposed to hypotonic conditions. Afterwards synaptic membranes and synaptic vesicles can be isolated from the lysate by sedimentation through a density gradient [146]. Such a procedure does not completely separate synaptic vesicles from the membrane. Indeed, lysis generates two different synaptic vesicle populations in the gradient. One vesicle pool migrates at lighter fractions and are identified as free vesicles (SV) and the other population accumulates at denser fractions. These latter synaptic vesicles co-migrate with proteins of the plasma membrane (SPM) as well as with proteins of the postsynaptic density and are therefore assumed to be docked vesicles [173].

Here, protease treated synaptosomes were lysed and the components separated on a 0.41.4 M continuous gradient. As previously reported, two vesicle populations were observed corresponding to free (SV) and docked vesicles (SPM) (Fig. 3.6). A more detailed western 
A $1.5 \mathrm{M}$

untreated

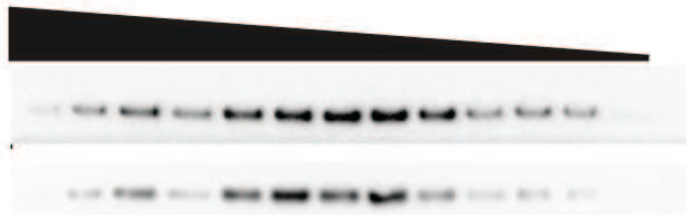

PSD95

\section{Synaptophysin}

+ trypsin

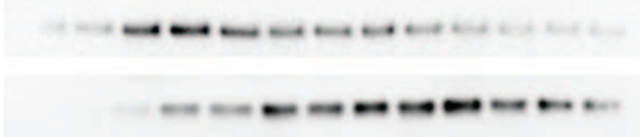

PSD95

Synaptophysin

B

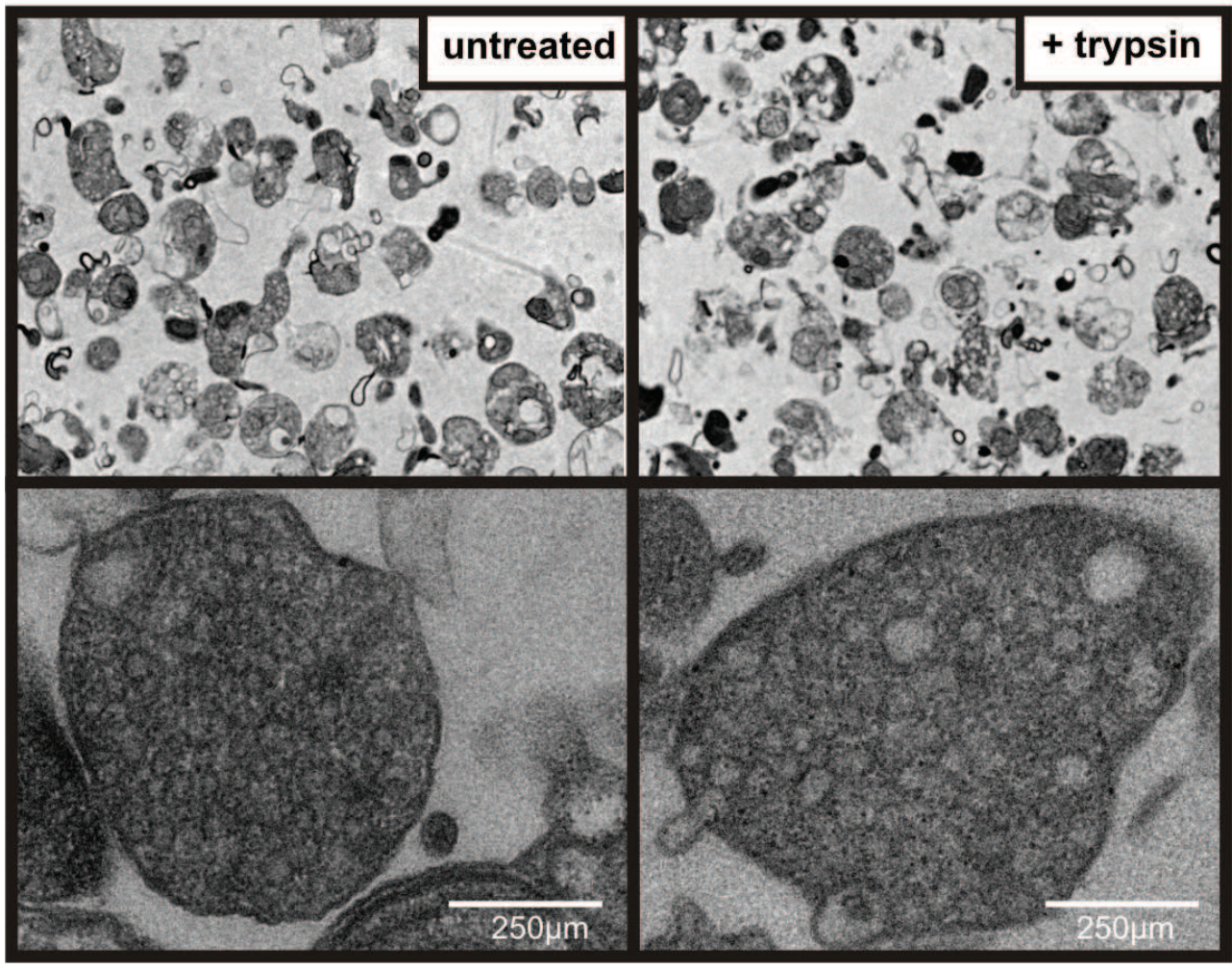

Figure 3.4: (A) Migration pattern of pre- and postsynaptic proteins in a continuous sucrose gradient. After protease treatment, synaptosomes were washed and loaded on a $0.75-1.5 \mathrm{M}$ sucrose gradient. Protease treatment of synaptosomes induced a migration shift of postsynaptic proteins. (B) Electron microscopy of untreated and trypsin treated synaptosomes from a continuous sucrose gradient. Fractions at $1.2 \mathrm{M}$ sucrose were diluted 1:5 in $5 \mathrm{mM}$ Hepes pH 7.4 and pelleted. No obvious change in synapotosome morphology was observed. 

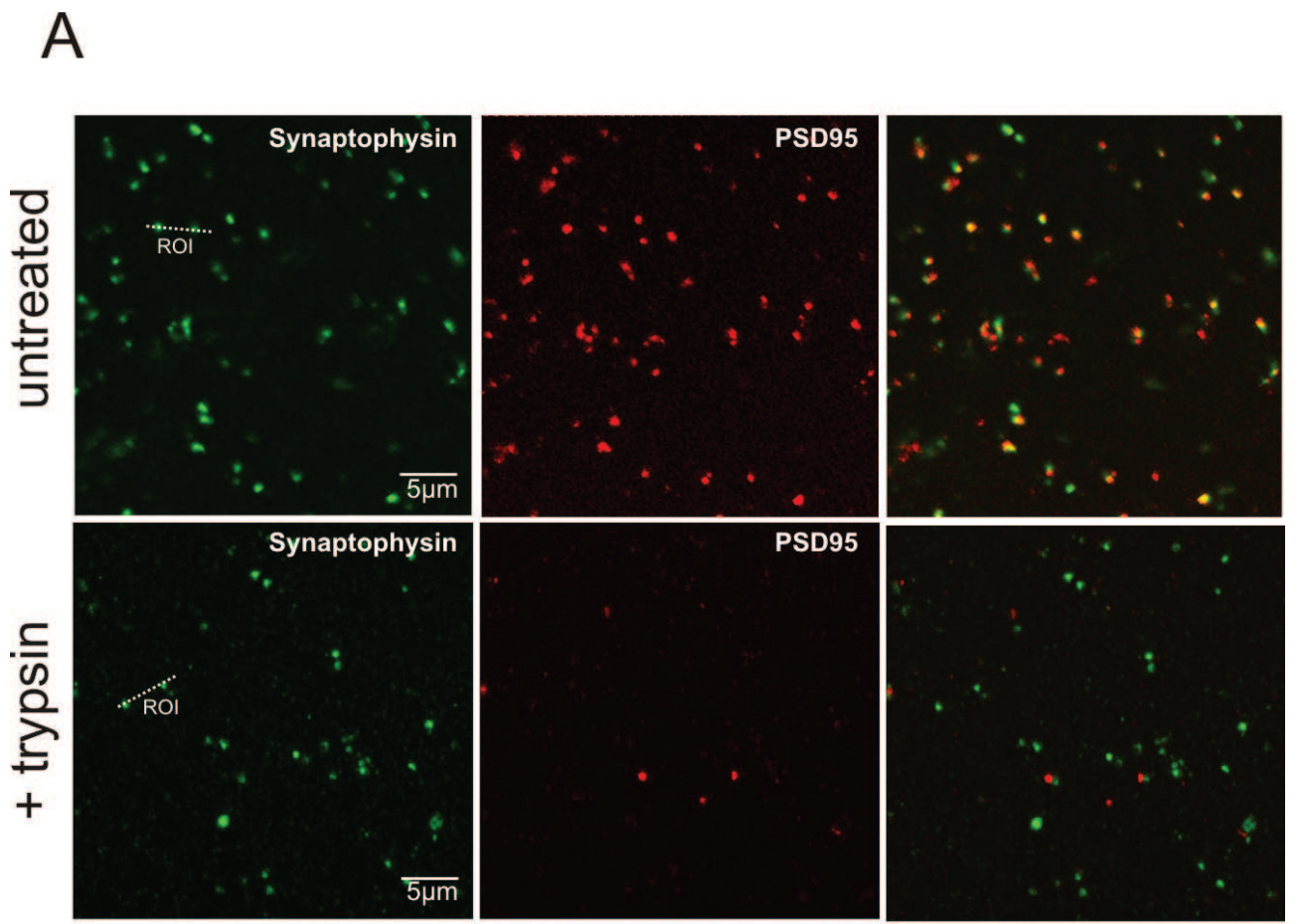

B
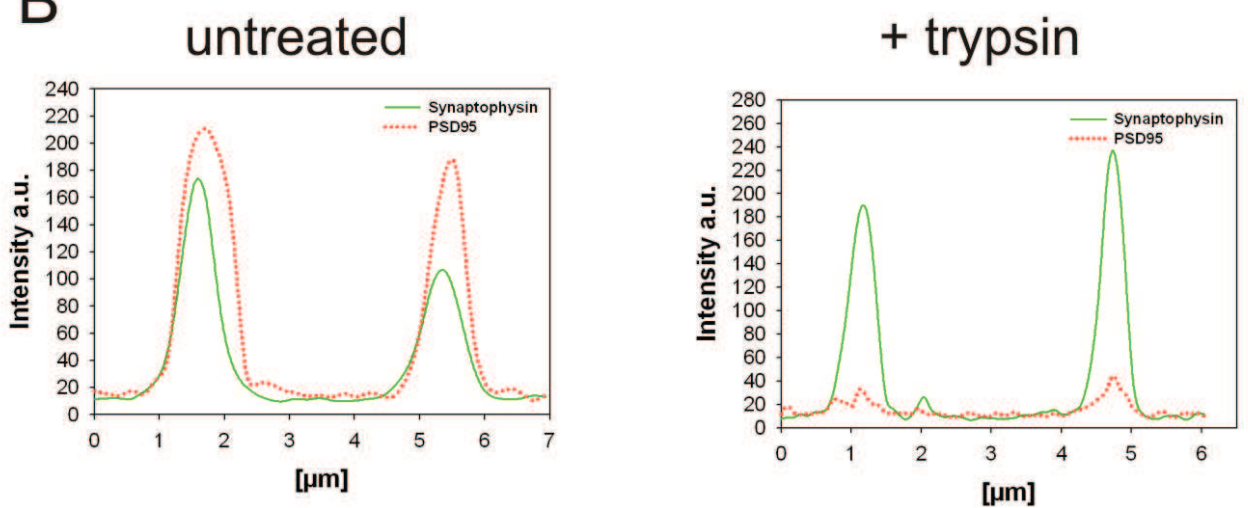

Figure 3.5: (A) Synaptosomal fractions at $1.2 \mathrm{M}$ sucrose in the gradient was pelleted on coverslips and preand postsynaptic specializations visualized by immuno-fluorescent staining with antibodies against synaptophysin (green) and PSD95 (red). Protease treatment reduced the detected postsynaptic signal while the presynaptic signal did not change. (B) Linescans showing co-localization of pre- and postsynaptic signal in untreated synaptosomes. Trypsinized samples were devoid of any postsynaptic signal. 
$1.4 \mathrm{M}$

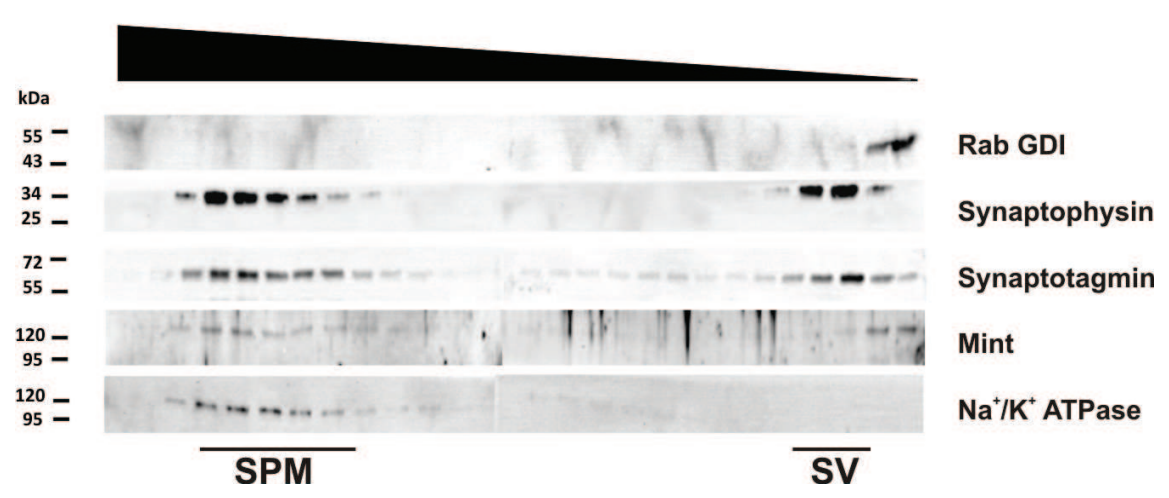

Figure 3.6: Gradient centrifugation of trypsinized and lysed synaptosomes displayed two synaptic vesicle populations. Docked vesicle co-migrated with active zone and plasma membrane marker.

blot analysis of the gradient fractions additionally revealed that the SPM fraction also contained components of the active zone (Mint, Munc13) and mitochondria (SDHA) (Fig. ??) . These proteins were greatly reduced or absent in the SV fractions. Thus, these data confirms that in addition to the attached vesicles, the presynaptic machinery remains coupled to the plasma membrane following hypotonic lysis.

Mitochondrial contamination in synaptic preparations has been an issue for several decades. A complete separation of these components according to their equilibrium buoyant densities is almost impossible. In the early seventies several groups published different protocols addressing this issue. Davis and Bloom increased the density of mitochondria by histochemically changing the succinic dehydrogenase with tetrazolium [174]. They could show a reduction of mitochondrial contamination of synaptic membranes, but the disadvantage of this protocol is that the procedure might change the stability of the synaptic complexes as it involves additional treatments and elevated temperatures. Another method to improve purity was published by Jones and Matus. They introduced a simultaneous sedimentation and flotation centrifugation step to separate mitochondria from synaptic membranes. Lysed synaptosomes were suspended in sucrose of intermediate density between both buoyant densities, resulting in flotation of SPM and a sedimentation of mitochondria [175]. Although being a true separation based on density, this protocol is limited with respect to the aim of this study, because separation of docked vesicles and free vesicles was not achieved. One of the earliest methods published is based on the $\mathrm{pH}$ during lysis. Cotman and Matthews showed that carrying out osmotic shock at alkaline pH greatly enhanced SPM purity [176]. However, these findings could not be reproduced in this work. As shown in fig. 3.7A, varying the $\mathrm{pH}$ from 7.5 to 8.5 did not change the distribution of mitochondria in the gradient. Under all conditions tested, the mitochondrial 
marker SDHA remained enriched in the SPM fractions. To evaluate if mitochondria in fact only co-migrate in the gradient or are actually an essential part of exocytotic sites at the plasma membrane, the isolated SPM fraction was analyzed for co-localization by immunofluorescent staining (Fig 3.7B). Images showed a significant overlap of the presynaptic active zone marker piccolo with the plasma membrane SNARE syntaxin 1. Interestingly, staining of syntaxin 1 appeared to be more dense than piccolo. This indicates that there are stretches of plasma membrane that contain SNAREs but no attached active zone. Based on the observed signals for piccolo and synaptotagmin, the abundance of active zone components to synaptic vesicles was comparable and the proteins were in close proximity as exemplified by the high degree of colocalization of these proteins. In contrast, signals for mitochondria and active zone proteins did not show any significant co-localization. Moreover, mitochondria were present in very high amounts. This supports the idea that mitochondria are only co-migrating with synaptic membranes as a consequence of the closeness of their sedimentation factors and are therefore heavily contaminating these fractions. Thus, additional purification steps for isolating docked vesicles were necessary.

\subsubsection{Immunoisolation of docked vesicles}

To simultaneously minimize mitochondrial contamination and concentrate docked vesicles, a immunoaffinity purification step was added to the existing protocol. The feasibility of this approach has been previously demonstrated [173], but has been modified in this study. Eupergit $\mathrm{C} 1 \mathrm{Z}$ beads covalently conjugated to antibodies specific for synaptophysin were used to isolate docked vesicle or free vesicles. Several buffer conditions including variations in salt concentration and supplements were tested to optimize yield while minimizing non-specific attachment of proteins. The best results were achieved using phosphate buffered saline (PBS) supplemented with bovine serum albumin (BSA) as a stabilizer (see Fig. 3.8, first panel). Under these conditions vesicles could be purified from the SPM fractions together with parts of the plasma membrane (as exemplified by the $\mathrm{Na}^{+} / \mathrm{K}^{+}$ATPase) and components of the active zone e.g. Munc13. Unfortunately, mitochondria were still present in the immunoisolates. Surprisingly, SDHA was also detected in the immunoisolates of control beads either coupled to the amino acid glycine $(\mathrm{G})$ or to unspecific anti-sheep $\operatorname{IgG}(\operatorname{IgG})$. This suggested that mitochondria might co-sediment as a result of the centrifugation step used to pellet the eupergit beads instead of actually being co-purified. To a lesser extent, unspecific binding of the vesicle proteins synaptophysin and synaptobrevin were observed. To test this hypothesis, the $g$ force of the centrifugation steps was reduced from $3400 \mathrm{~g}$ (6000 rpm)to $400 \mathrm{~g}$ (2000 rpm). With IgG or glycine coupled beads, unspecific binding of synaptophysin and synaptobrevin was almost 
A
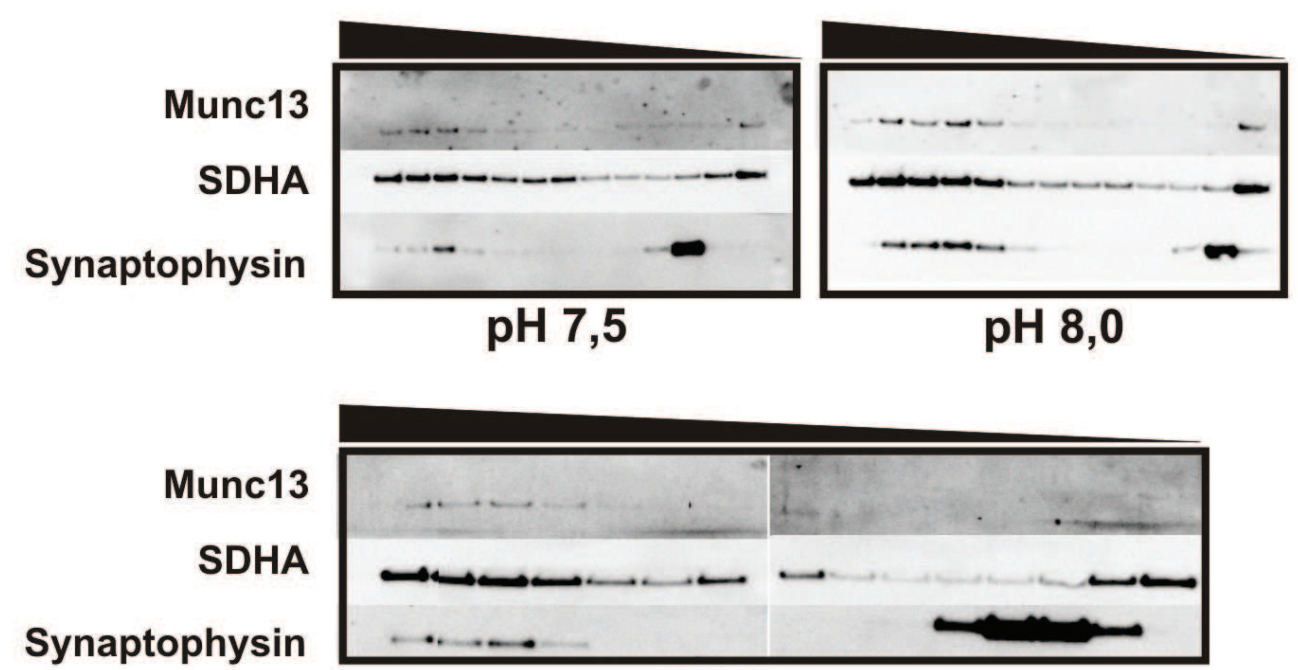

$\mathrm{pH} \mathrm{8,5}$

B
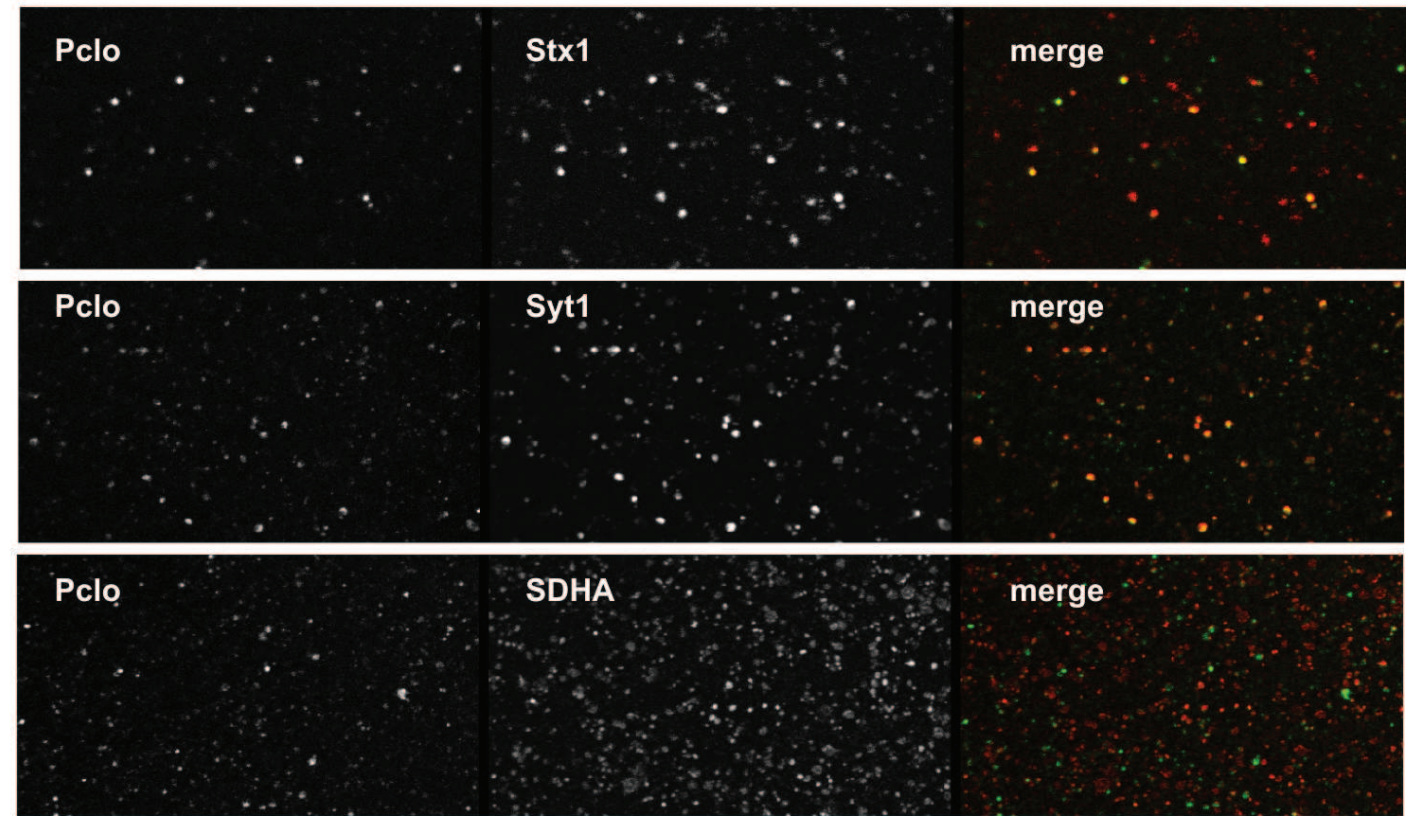

Figure 3.7: (A) Co-migration of mitochondria and docked vesicles in a sucrose gradient is not $\mathrm{pH}$ dependant. Synaptosomes were osmotically lysed at $\mathrm{pH} 7.5, \mathrm{pH} 8.0$ or $\mathrm{pH} 8.5$ and its components separated on a 0.4.-1.3M continuous sucrose geradient. Migration patterns for mitochondria and synaptic plasma membranes did not change despite varying the $\mathrm{pH}$ used during the lysis. (B) Immunofluorescent staining of SPM fractions. The active zone protein piccolo showed a high co-localization with the synaptic vesicle protein synaptotagmin. Partial overlap of piccolo with the plasma membrane SNARE syntaxin 1 was also observed. Fractions were heavily contaminated with mitochondria. 
completely abolished and the signal for SDHA was greatly diminished. Nevertheless, similar amounts of mitochondria were still co-purified with beads immobilized to the antibody for synaptophysin (Fig. 3.8, 2nd panel). Maybe mitochondria are attached to the synaptic plasma membrane by other forces such as lipid-lipid interactions. Because the mitochondrial proteome is well characterized, it allowed to eliminate mitochondrial contamination from the generated data.

Free synaptic vesicles could be isolated from the corresponding fractions using the same protocol (Fig. 3.8, 3rd panel). As expected, only vesicle proteins were purified (VGlut1, synaptophysin and synaptobrevin). Neither the plasma membrane ATPase nor active zone components were detected in the immunoisolates. Mitochondrial contamination was still observed, but to a significantly lesser extent than in docked vesicles. In this work quantitative mass spectrometry was used to compare the protein composition of docked and free vesicles. Importantly, the amount of immunoisolated vesicles from the docked and free vesicle fraction were required to be approximately equal. This allows proteins enriched in the docked fraction to be confidently identified while the ratios of SV proteins in both fractions to be 1:1. To achieve this, input amounts of docked and free vesicle fractions were adjusted according to the chemiluminescence signal intensity of synaptophysin, synaptobrevin and VGlut1 in the immunoisolates (Fig. 3.8 last panel). Using twice the amount of the docked vesicle fraction compared to free vesicles as a starting material resulted in approximately same amount of synaptophysin, synaptobrevin and VGlut1. 


\begin{tabular}{|c|c|c|c|c|c|}
\hline 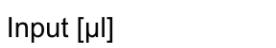 & 600 & 600 & 600 & 600 & 300 \\
\hline$g$ force & 3400 & 400 & 400 & 400 & 400 \\
\hline conjugated Antibody & $S \quad G \lg G$ & $S \quad G \lg G$ & $S G \lg G$ & $S \lg G$ & $S \lg G$ \\
\hline Munc13 & $--\cdots$ & - & & & \\
\hline $\mathrm{Na}^{+} / \mathrm{K}^{+}$ATPase & $=-\cdots$ & - & 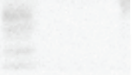 & - & $=$ \\
\hline SDHA & $-\infty$ & $=-$ & & & \\
\hline VGlut1 & Ext kn & $=0$ & $y=1$ & & \\
\hline \multicolumn{6}{|l|}{ Synaptophysin } \\
\hline \multicolumn{6}{|l|}{ Synaptobrevin } \\
\hline & SPM & & SV & SPM & SV \\
\hline
\end{tabular}

Figure 3.8: Docked vesicles (SPM) and free vesicles (SV) were immunoisolated from the corresponding fractions of the sucrose gradient using Eupergit $\mathrm{C} 1 \mathrm{Z}$ beads comjugated to antibodies specific for synaptophysin. For unspecific binding controls, Eupergit $\mathrm{C} 1 \mathrm{Z}$ were coupled to anti-sheep IgG or inactivated by glycine. SPM immunoisolates contained in addition to synaptic vesicles plasma membrane proteins $\left(\mathrm{Na}^{+} / \mathrm{K}^{+}\right.$ATPase) and active zone components (Munc13). Low speed centrifugation minimized unspecific background but did not remove mitochondrial contamination. Input of SPM and SV were adjusted to similar amounts of synaptic vesicle proteins detected in the immunoisolates. 


\subsection{Quantitative comparison of a docked and free vesicle proteome}

The proteomes of the immunoisolates containing free and docked vesicle fractions were compared using an isobaric tag for relative and absolute quantification (iTRAQ) in combination with tandem mass spectrometry (LC-MS/MS). This quantitation method was developed by Ross et al. and it is designed to compare relative protein amounts in complex mixtures [141]. In detail, the iTRAQ reagent places an isobaric mass tag to reactive amino groups of the tryptic peptides generated after solubilisation and digestion of the sample. In this case, the docked vesicle fraction was labeled with a tag of $\mathrm{m} / z 117$ and free vesicles with $\mathrm{m} / \mathrm{z} 116$. Both samples were combined after the labeling and analyzed. The labeled peptides are chromatographically indistinguishable, but upon peptide fragmentation during the MS/MS analysis they yield different reporter ions of the masses $\mathrm{m} / z 117$ and $\mathrm{m} / \mathrm{z} 116$. The intensity of these reporter ions can be used to calculate the relative abundance of individual proteins. To reduce the complexity of the sample, the digested samples were pre-fractionated by strong cation exchange chromatography using increasing salt concentration steps. Afterwards the fractions were separately analyzed by reverse phase LC-MS/MS (see Fig. 3.9A).

In total, 506 proteins were identified from both fractions. Not surprisingly, a substantial portion of 217 proteins (42\%) originated from the mitochondria according to NCBI and MitoCarta [177]. These proteins were not considered in the following analysis and are listed in the appendix. The remaining 289 proteins were investigated in greater detail and sorted into different functional groups according to their subcellular localization or molecular function ( Fig 3.9B). The clustering of functional groups reflect the existence of several molecular mechanisms participating in the regulation of synaptic transmission.

Remarkably, only three exclusively postsynaptic proteins were identified (PSD, syngap 1, kalirin). Furthermore, in addition to mitochondria (as mentioned above), only minor contamination of other membranous organelles was detected. This minor contamination includes molecules involved in nucleotide metabolism, protein synthesis, metabolic enzymes, chaperones and proteins located at centrosomes, ER or Golgi (see appendix).

More than 30 hitherto uncharacterized proteins were detected, many of which are integral membrane proteins while others are identified purely by gene prediction. One of these proteins has been characterized in some detail in 3.4

In summary, the proteome presented in this work provides detailed insights into the molecular composition of synaptic vesicle docking sites and the plasma membrane underlying these sites. A static picture of a synapse assembled by the proteins identified in this screen is presented at the end of this section (Fig. 3.10). 
A

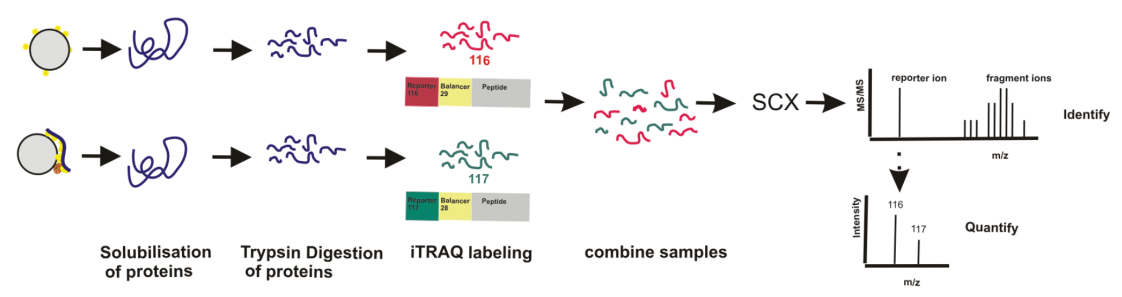

B

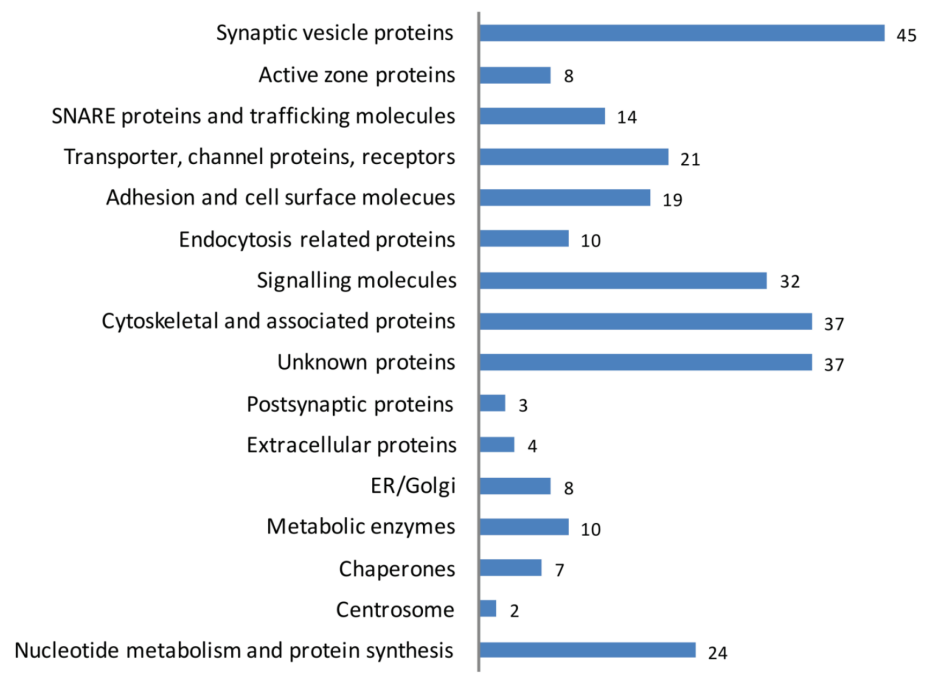

Figure 3.9: (A) Schematic overview of the iTRAQ experiment. (B) Proteins identified by iTRAQ analysis of docked and free vesicles were grouped according to their subcellular localization or molecular function.

\subsubsection{Synaptic vesicle proteins}

Synaptic vesicle proteins constitute the largest group of proteins identified. These proteins were detected in same amounts in both fractions as evidenced by a 1:1 ratio of the reporter ions $\mathrm{m} / \mathrm{z}, 117$ and $\mathrm{m} / \mathrm{z}$ 116. Synaptic vesicles are one of the best characterized organelles in biology. A complete quantitative description of the protein composition of purified SVs has already been published [138] and was used as a reference for the analyzed data. The vacuolar ATPase, which is essential for the acidification of synaptic vesicles, has been determined to be present in one or two copies per vesicle [138, 137]. The vATPase is a large multi-subunit complex, the peripheral membrane domain V1 is about $640 \mathrm{kDa}$ in size. Due to its enormous size, great variations in the abundance of the vATPase on SVs are unlikely. Therefore, the iTRAQ protein ratio was calculated by normalizing the amount of the vATPase in the docked vesicle fraction against the amount in the free vesicle fraction. As shown in table 3.1, all major synaptic 
vesicle proteins were identified. This includes neurotransmitter transporters such as VGlut1, VGlut2 and VGAT, integral SV proteins like synaptophysin, the synaptotagmins $(1,2,5), \mathrm{SV} 2 \mathrm{a}$ and $\mathrm{b}$, and membrane associated proteins such as synapsins $(1,3)$ as well as a number of Rab GTPases. Mover [178] and MAL2 [130] (proteins only recently identified as synaptic vesicle components) were detected. Importantly, almost all vesicle proteins were present in equal amounts in both fraction as exemplified by an iTRAQ ratio in the range of 1. Taking a twofold increase in the ratio as a cut off for enrichment, only Rab proteins showed a slight enrichment in the docked vesicle fraction. Rab GTPases are molecular switches that cycle between an active membrane attached state, in this case synaptic vesicles, and the cytosol depending on the bound nucleotide (for review see [179]). Unlike docked vesicles that require a membrane bound active Rab GTPase, not all free vesicles might contain a bound Rab protein. In fact, Pavlos et al. showed that Rab

The Rab family consists of over 60 members and a great number of Rab proteins were originally found on purified SVs that are involved in completely different trafficking pathways [138]. Recently, Pavlos et al. could determine the core Rab machinery using a quantitative analysis of synaptic vesicle Rabs [21]. Rab27b and the Rab3 isoforms are the major exocytotic, while Rab4b, Rab5a/b, Rab11 and Rab14 represent the endocytotic Rabs of synaptic vesicles. In line with this findings, the Rab proteins identified in this work represent the core Rab machinery. However, Rab proteins are very redundant in their sequence. Therefore only unique peptides were used for protein quantification.

\subsubsection{Active zone proteins}

In contrast to the SV proteins, active zone components were highly enriched in the docked vesicle fraction. Indeed, these proteins exhibited iTRAQ ratios of greater than six-fold (as seen for bassoon and Rim1) or were exclusively found in the SPM fraction.

Almost all established active zone components were detected in the docked vesicle fraction. Importantly, the identification of these proteins validated our purification approach as being appropiate for the analyses of the presynaptic release machinery. The AZ proteins identified included the multidomain scaffolding proteins piccolo, bassoon and CASK, the cytomatrix organizer ERC2 and proteins of the liprin family. Among the identified active zone proteins, a distinctive network of protein-protein interactions exists (as described in the introduction 1.2). Of the core active zone proteins, only Munc13, MALS and Mint1 could not be identified by mass spectrometry. However, the presence of Munc13 in these fractions has been proven by immunoblotting (see Fig 3.8 ). Munc13 appears to be difficult to analyze by mass spectrometry as noticed in other proteomic approaches [173, 180]. This "loss" of Munc13 might result from 


\begin{tabular}{|c|c|c|c|c|c|}
\hline \multicolumn{6}{|c|}{ Synaptic vesicle proteins } \\
\hline GI number & $\begin{array}{l}\text { Gene } \\
\text { symbol }\end{array}$ & Name & $\#$ & $\begin{array}{r}\text { iTRAQ } \\
\text { ratio }\end{array}$ & std \\
\hline gi|16758202 & Rab27b & RAB27B & 2 & 2.6 & 0.6 \\
\hline gi|16758368 & Rab14 & RAB14 & 3 & 2.5 & 0.7 \\
\hline gi|45433570 & Rab1a & RAB1A & 3 & 2.3 & 0.1 \\
\hline gi|61556789 & Rab35 & RAB35 & 3 & 2.2 & 0.2 \\
\hline gi|61098195 & Rab3a & RAB3A & 3 & 2.2 & 0.3 \\
\hline gi|13929006 & Rab2a & RAB2A & 3 & 2.2 & 0.4 \\
\hline gi|9507159 & Syn1 & synapsin I isoform a & 3 & 1.9 & 0.9 \\
\hline gi|8394389 & Syn3 & synapsin III & 2 & 1.8 & 0.0 \\
\hline gi|158749626 & Scamp1 & secretory carrier membrane protein 1 & 3 & 1.8 & 1.2 \\
\hline gi|61889071 & Rab10 & RAB10, member RAS oncogene family & 3 & 1.7 & 0.1 \\
\hline gi|13592037 & Rab3b & RAB3B, member RAS oncogene family & 2 & 1.7 & 0.5 \\
\hline gi|77404242 & Syn2 & synapsin II isoform 1 & 3 & 1.6 & 0.2 \\
\hline gi|74271849 & Slc6a17 & neurotramsmitter transporter NTT4 & 3 & 1.5 & 0.2 \\
\hline gi|109499663 & Atp8a1 & Aminophospholipid transporter (APLT) 8A, member 1 & 3 & 1.4 & 0.1 \\
\hline gi|6981624 & Syt2 & synaptotagmin II & 3 & 1.4 & 0.2 \\
\hline gi|9507171 & Syt5 & synaptotagmin $\mathrm{V}$ & 2 & 1.3 & 0.2 \\
\hline gi|148356226 & Syt1 & synaptotagmin 1 & 3 & 1.1 & 0.1 \\
\hline gi|109465077 & Scamp3 & Secretory carrier membrane protein 3 & 2 & 1.1 & 0.1 \\
\hline gi|13162361 & Dnajc5 & cysteine string protein & 3 & 1.1 & 0.1 \\
\hline gi|40786463 & Atp6v1d & ATPase, $\mathrm{H}+$ transporting, lysosomal V1 subunit $\mathrm{D}$ & 3 & 1.1 & 0.0 \\
\hline gi|16758754 & Atp6v1f & ATPase, $\mathrm{H}$ transporting, lysosomal $\mathrm{V} 1$ subunit $\mathrm{F}$ & 3 & 1.0 & 0.1 \\
\hline gi|58865560 & Atp6v1c1 & ATPase, $\mathrm{H}+$ transporting, lysosomal V1 subunit $\mathrm{C} 1$ & 3 & 1.0 & 0.2 \\
\hline gi|38454230 & Atp6v1e1 & vacuolar H+ ATPase E1 & 3 & 1.0 & 0.1 \\
\hline gi|58865424 & Atp6v0d1 & ATPase, $\mathrm{H}+$ transporting, lysosomal $38 \mathrm{kDa}$, V0 subunit $\mathrm{d} 1$ & 3 & 0.9 & 0.0 \\
\hline gi|77627990 & Atp6v0a1 & ATPase, $\mathrm{H}+$ transporting, lysosomal V0 subunit a1 & 3 & 0.9 & 0.0 \\
\hline gi|62078587 & Atp6v1h & ATPase, $\mathrm{H}+$ transporting, lysosomal V1 subunit $\mathrm{H}$ & 3 & 0.9 & 0.0 \\
\hline gi|109493234 & Atp6v1a1 & ATPase, $\mathrm{H}+$ transporting, $\mathrm{V} 1$ subunit $\mathrm{A}$, isoform 1 & 3 & 0.9 & 0.0 \\
\hline gi|47059104 & Atp6v1g2 & ATPase, $\mathrm{H}+$ transporting, lysosomal V1 subunit G2 & 3 & 0.9 & 0.1 \\
\hline gi|17105370 & Atp6v1b2 & vacuolar H+ATPase B2 & 3 & 0.9 & 0.0 \\
\hline gi|13929110 & Atp6ap1 & ATPase, $\mathrm{H}+$ transporting, lysosomal accessory protein 1 & 3 & 0.9 & 0.0 \\
\hline gi|16758166 & Slc17a6 & VGlut2 & 3 & 0.9 & 0.1 \\
\hline gi|16758726 & Slc17a7 & VGlut1 & 3 & 0.9 & 0.1 \\
\hline gi|17105360 & Sv2b & synaptic vesicle glycoprotein $2 \mathrm{~b}$ & 3 & 0.9 & 0.1 \\
\hline gi| 148747227 & Sv2a & synaptic vesicle glycoprotein $2 a$ & 3 & 0.8 & 0.0 \\
\hline gi|160333093 & Tprgl & mover & 3 & 0.8 & 0.2 \\
\hline gi|13929106 & Slc32a1 & VGat & 3 & 0.8 & 0.1 \\
\hline gi|61557417 & Slc30a3 & zinc transporter $\mathrm{ZnT}-3$ & 3 & 0.8 & 0.0 \\
\hline gi|77157795 & Mal2 & MAL2 proteolipid protein & 3 & 0.8 & 0.0 \\
\hline gi|13027428 & Synpr & synaptoporin & 2 & 0.8 & 0.2 \\
\hline gi|18677757 & Atp6v0c & ATPase, $\mathrm{H}+$ transporting, lysosomal $16 \mathrm{kDa}, \mathrm{V} 0$ subunit c & 3 & 0.7 & 0.1 \\
\hline gi|6981622 & Syp & synaptophysin & 3 & 0.7 & 0.0 \\
\hline gi|13929020 & Scamp5 & secretory carrier membrane protein 5 & 3 & 0.6 & 0.0 \\
\hline gi|9507167 & Syngr1 & synaptogyrin 1 & 3 & 0.6 & 0.0 \\
\hline gi|157819371 & Syngr3 & synaptogyrin 3 & 3 & 0.5 & 0.0 \\
\hline
\end{tabular}

Table 3.1: The table shows the synaptic vesicle proteins identified in the docked and free vesicle fraction. \# indicates the number of times the protein was detected in 3 biological replicates. The iTRAQ ratio was calculated as $m / z 117$ divided by $m / z 116$ (docked/free vesicles). 


\begin{tabular}{|c|c|c|c|c|c|}
\hline \multicolumn{6}{|c|}{ Active zone proteins } \\
\hline GI number & $\begin{array}{l}\text { Gene } \\
\text { symbol }\end{array}$ & Name & \# & $\begin{array}{l}\text { iTRAQ } \\
\text { ratio }\end{array}$ & std \\
\hline gi|115599477 & Cask & calcium/calmodulin-dependent serine protein kinase & 2 & SPM & \\
\hline gi|25140983 & Erc2 & ELKS/RAB6-interacting/CAST family member 2 & 3 & SPM & \\
\hline gi|109497902 & Ppfia4 & Liprin- $\alpha 4$ & 3 & SPM & \\
\hline gi|10048483 & Pclo & piccolo isoform 1 & 2 & SPM & \\
\hline gi|157824053 & PPFIA2 & Liprin- $\alpha 2$ & 2 & SPM & \\
\hline gi|158749559 & Bsn & Bassoon & 3 & 7.4 & 0.8 \\
\hline gi|16306470 & Rims1 & RIM 1 & 3 & 6.3 & 0.4 \\
\hline gi|213972596 & Ppfibp2 & Liprin- $\beta 2$ & 3 & 0.4 & 0.1 \\
\hline
\end{tabular}

Table 3.2: Active zone proteins identified in the iTRAQ analysis. Proteins with an iTRAQ ratio of "SPM" have only been identified in the docked vesicle fraction and could therefore not be quantified.

the high insolubility of Munc13 in detergents other than SDS or from a masking of the peptides in the MS spectrum by other more abundant peptides.

In our proteomic analysis, neither Mint1 nor MALS could be identified. They may have been missed for the same reasons as for Munc13 or they are really absent in this fraction. Genetic studies of MALS showed, that knock out of this protein results in aberrant EPSC amplitudes and an accelerated synaptic depression after high frequency stimulation. This lead to the assumption that this complex is involved in replenishing the readily releasable pool from the reserve pool [112]. In this respect, the complex possibly disassembles after docking of the vesicle to the plasma membrane and as a consequence is not detected here.

Along with the $\alpha$-liprins, also the less characterized isoform liprin- $\beta 2$ was detected, but did not show an enrichment in the docked vesicle fraction (iTRAQ ratio of 0.5 ). Thus, this protein appears to have a different localization as $\alpha$-liprins and presumably is not involved in docking of vesicles to the plasma membrane

\subsubsection{SNARE proteins and trafficking molecules}

In addition to synaptic vesicles and active zone components, all known components of the neuronal fusion machinery were identified with this approach.

Vesicle fusion in general is mediated by different groups of proteins. SNARE proteins form the minimal machinery for membrane fusion and SM proteins bind trans-SNARE complexes thereby regulating their fusogenic activity. In addition complexin is thought to neurotransmitter release by grappling zippered SNARE complexes and releasing them upon calcium influx. In agreement with current literature it was shown here that the neuronal t-SNAREs SNAP25 and 
syntaxin 1a and $\mathrm{b}$ were enriched in the SPM fraction, whereas the v-SNAREs Vamp1 and Vamp2 had iTRAQ ratios of approximately 1 and are localized to synaptic vesicles (see table 3.3). Furthermore the synaptic SM protein Munc18 and complexin are enriched in the docked vesicle fraction.

Interestingly, a component of the SNARE recycling machinery was found as well. NSF, a member of the AAA family of ATPases, takes action after the fusion event and disassembles cis-SNARE complexes. However, the NSF adaptor protein $\alpha$-SNAP was not detected.

Apart from the neuronal SNAREs, only one non-neuronal SNARE was detected. Syntaxin 16 and its associated SM protein vps45 were enriched in the docked vesicle fraction. Synatxin 16 is ubiquitously expressed, but mainly localizes at the trans-Golgi network [181].

\begin{tabular}{|c|c|c|c|c|c|}
\hline GI number & Gene symbol & Name & $\#$ & $\begin{array}{r}\text { iTRAQ } \\
\text { ratio } \\
\end{array}$ & std \\
\hline gi|109471437 & Stx16 & Syntaxin-16 (Syn16) isoform 1 & 2 & SPM & \\
\hline gi|25742604 & Vps45 & vacuolar protein sorting 45 homolog & 2 & SPM & \\
\hline gi|9507127 & Snip & SNAP25-interacting protein & 3 & 5.9 & 1.9 \\
\hline gi|12408324 & Cplx1 & complexin 1 & 2 & 5.3 & 2.3 \\
\hline gi|13591882 & Snap25 & synaptosomal-associated protein 25 & 3 & 4.2 & 0.1 \\
\hline gi|6981600 & Stx1b2 & syntaxin $1 \mathrm{~B} 2$ & 3 & 3.4 & 0.1 \\
\hline gi|13489067 & Nsf & $\mathrm{N}$-ethylmaleimide-sensitive factor & 3 & 3.3 & 1.6 \\
\hline gi|33667087 & Stx1a & syntaxin $1 \mathrm{~A}$ (brain) & 3 & 3.0 & 0.1 \\
\hline gi|219275534 & Vps13a & vacuolar protein sorting 13 homolog $\mathrm{A}$ & 2 & 2.8 & 0.1 \\
\hline gi|13027430 & Wdr7 & rabconnectin 3 beta & 2 & 2.5 & \\
\hline gi|6981602 & Stxbp1 & Munc18-1 & 3 & 2.3 & 0.2 \\
\hline gi|62079163 & Atg9a & ATG9 autophagy related 9 homolog A & 3 & 1.2 & 0.5 \\
\hline gi|76443677 & Vamp1 & vesicle-associated membrane protein 1 & 3 & 0.9 & 0.1 \\
\hline gi|6981614 & Vamp2 & Synaptobrevin & 3 & 0.7 & 0.0 \\
\hline
\end{tabular}

Table 3.3: The neuronal fusion machinery consisting of the SNAREs syntaxin 1a/b, SNAP25 and Vamp2, Munc18 and complexin was identified in the analyzed fractions. All proteins except for the v-SNAREs Vamp1 and Vamp2 were enriched in the docked vesicle fraction.

\subsubsection{Transporter, channel proteins and receptors}

The presynaptic membrane contains an extensive array of molecules that are involved in the signaling process across the synapse. Here we showed that the stretches of the plasma membrane purified with the docked vesicles contain a number of transporters and channels that are involved in calcium homeostasis, modulation of synaptic strength, $\mathrm{pH}$ maintenance and neurotransmitter clearance at the nerve terminal. 
Transporter, channel proteins, receptors

\begin{tabular}{|c|c|c|c|c|c|}
\hline GI number & Gene symbol & Name & \# & $\begin{array}{r}\text { ITRAQ } \\
\text { ratio }\end{array}$ & std \\
\hline gi|31542335 & Cacna2d1 & calcium channel, voltage-dependent, $\alpha 2 / \delta$ subunit 1 & 2 & SPM & \\
\hline gi|6978583 & Cacnb3 & calcium channel, voltage-dependent, $\beta 3$ subunit & 2 & SPM & \\
\hline gi|16758108 & Hen1 & $\begin{array}{l}\text { hyperpolarization-activated cyclic nucleotide-gated } \\
\text { potassium channel } 1\end{array}$ & 2 & SPM & \\
\hline gi|13929184 & Kcnma1 & $\begin{array}{l}\text { potassium large conductance calcium-activated } \\
\text { channel, subfamily } \mathrm{M} \text {, alpha member } 1\end{array}$ & 2 & SPM & \\
\hline gi|155369700 & TIr8 & toll-like receptor 8 & 2 & SPM & \\
\hline gi|6981558 & Slc9a1 & sodium/hydrogen exchanger Nhe1 & 2 & SPM & \\
\hline gi|62644838 & Slc27a4 & fatty acid transporter, member 4 & 3 & SPM & \\
\hline gi|157817045 & Clen6 & chloride channel 6 & 2 & SPM & \\
\hline gi|13242269 & Slc6a1 & GABA transporter protein & 2 & SPM & \\
\hline gi|9507115 & Slc1a3 & EAAT1 & 2 & SPM & \\
\hline gi|78126161 & Slc1a2 & EAAT2 & 2 & 8.9 & 1.9 \\
\hline gi|148747253 & Atp1b1 & $\mathrm{Na}+/ \mathrm{K}+-$ ATPase beta 1 subunit & 3 & 8.4 & 1.2 \\
\hline gi|6978543 & Atp1a1 & $\mathrm{Na}+/ \mathrm{K}+-\mathrm{ATPase}$ alpha 1 subunit & 3 & 7.8 & 0.7 \\
\hline gi|6978547 & Atp1a3 & $\mathrm{Na}+/ \mathrm{K}+-$ ATPase alpha 3 subunit & 3 & 7.3 & 0.6 \\
\hline gi|55925610 & Itpr1 & inositol 1,4,5-triphosphate receptor, type 1 & 3 & 6.9 & 1.0 \\
\hline gi|16758008 & Atp2b1 & plasma membrane calcium ATPase 1 & 3 & 6.3 & 2.8 \\
\hline gi|6978557 & Atp2b2 & plasma membrane calcium ATPase 2 & 2 & 6.2 & 3.9 \\
\hline gi|148747140 & Slc2a3 & glucose transporter GLUT3 & 2 & 4.7 & 0.3 \\
\hline gi|17530967 & Slc8a2 & sodium/calcium exchanger Ncx2 & 3 & 4.6 & 1.1 \\
\hline gi|19705463 & Slc12a5 & Neuronal potassium-chloride transporter & 3 & 4.2 & 1.2 \\
\hline gi|47576439 & Olr1589 & olfactory receptor Olr1589 & 2 & 2.1 & \\
\hline
\end{tabular}

Table 3.4: The docked vesicle fraction contains transporters and channels proteins that are involved in calcium homeostasis, modulation of synaptic strength, $\mathrm{pH}$ maintenance and neurotransmitter clearance at the nerve terminal.

Voltage-gated calcium channels initiate the release of neurotransmitters at the presynaptic nerve terminal. Mainly N-type and P/Q-type calcium channels are responsible for presynaptic calcium influx at conventional synapses. Calcium channels are composed of a poreforming $\alpha 1$ subunit that determines the pharmacological classification, and four distinct auxilliary subunits: an intracellular $\beta$-subunit, a disulfide linked complex of $\alpha 2 \delta$ and a $\gamma$-subunit. In this work, the subunits $\beta$ and $\alpha 2 \delta$ were detected, but not the transmembrane $\alpha 1$ subunit. This made it impossible to distinguish between L-, N- and P/Q-type calcium channels.

Second, calcium-dependent potassium channels (also known as BK channels) modulate synaptic transmission by altering the duration of presynaptic action potentials [182]. The BK channel Kcnma1 which localizes at presynaptic terminals [183, 184] was identified in this analysis as well as the hyperpolarization-activated cyclic nucleotide-gated potassium channel (HCN1). HCN1 recently was reported to be present at active zones of asymmetric synapses [185]. 
Importantly, calcium has to be actively removed from the cell after neuronal excitation. Both of the calcium clearance systems were enriched in our docked vesicle fraction. The plasma membrane $\mathrm{Ca}^{2+}$ - ATPase (PMCA) and the plasma membrane $\mathrm{Na}^{+} / \mathrm{Ca}^{2+}$ exchanger (NCX) exchanger extrude calcium from the cell and have been previously reported to enrich in synaptic plasma membrane preparations. In fact, PMCA is known to cluster to neurotransmitter release sites [186].

In addition to the calcium and calcium-dependent machinery, presynaptic terminals require a $\mathrm{pH}$ regulatory system to control intra- and extracellular $\mathrm{pH}$-changes. Here, we identified the sodium/hydrogen exchanger NHE1. NHE1 removes $\mathrm{H}^{+}$from synaptosomes [187] and its inhibition changes neurotransmitter release in dissociated hippocampal neurons [188].

Finally, also components of the neurotransmitter recycling machinery were found in the SPM fraction. GAT1 is the predominant transporter responsible for the re-uptake of GABA from the synaptic cleft, and is localized on presynaptic terminals of GABAergic inhibitory neurons [189]. Furthermore, the sodium-dependent glutamate transporters EAAT1 and EAAT2 were detected. Although these transporters are mainly distributed on glial plasma membranes [190], these transporters are also detected in synaptosomes and appear to directly associate with the $\mathrm{Na}^{+} / \mathrm{K}^{+}$ATPase [191].

\subsubsection{Adhesion and cell surface molecules}

Synaptosomes were treated with proteases during the purification process resulting in a digestion of proteins on the surface of the plasma membrane. This makes the identification of adhesion and cell surface molecules difficult and possibly incomplete. Nevertheless, cell adhesion molecules usually contain an intracellular domain that is expected to be protected from proteases. Therefore, several known plasma membrane proteins and adhesion molecules bridging the synaptic cleft were found in the docked vesicle fractions (see table 3.5).

Proteins involved in neurite formation and neuronal morphology were detected. The neuronal Growth-Associated Protein 43 (GAP43) plays a role in neuronal development [192] and is one of the most abundant proteins in growth cones [193]. Glycoproteins M6a and M6b are related proteolipid proteins. M6a is an axonal component of glutamatergic neurons with a suggested role in neurite outgrowth and stress response [194, 195]. Also Thy-1 enriches in synaptosomes [196] and modulates neurite outgrowth, but it additionally requires calcium influx through both $\mathrm{N}$ - and L-type calcium channels [197].

Among the identified synaptic adhesion molecules were NCAM, Neurexin4, CAM3, neuroplastin, sirpa and CD47. The latter finding is consistent with sirpa having a role in organizing the clustering vesicles mediated by its interaction with CD47 [198]. Although synaptic 


\begin{tabular}{|c|c|c|c|c|c|}
\hline \multicolumn{6}{|c|}{ Adhesion and cell surface molecules } \\
\hline Gl number & $\begin{array}{l}\text { Gene } \\
\text { symbol }\end{array}$ & Name & $\#$ & $\begin{array}{r}\text { iTRAQ } \\
\text { ratio }\end{array}$ & std \\
\hline gi|14091742 & Cntnap1 & Neurexin 4 & 2 & SPM & \\
\hline gi|157817081 & Ctnna2 & catenin alpha 2 & 2 & SPM & \\
\hline gi| 157820047 & Ctnnd1 & catenin delta 1 & 2 & SPM & \\
\hline gi|109464562 & Ctnnd2 & Neurojungin & 3 & SPM & \\
\hline gi| 109478967 & Crim1 & Cysteine-rich motor neuron 1 protein & 2 & SPM & \\
\hline gi|30017437 & Gpm6a & glycoprotein m6a & 3 & SPM & \\
\hline gi|20301986 & Gpm6b & glycoprotein $\mathrm{m} 6 \mathrm{~b}$ & 2 & SPM & \\
\hline gi|8850221 & Hpca & hippocalcin & 2 & SPM & \\
\hline gi|157817019 & Pkp4 & plakophilin 4 & 2 & 9.7 & 1.1 \\
\hline gi|8393415 & Gap43 & growth associated protein 43 & 3 & 8.9 & 1.3 \\
\hline gi|46048609 & Ctnnb1 & catenin beta 1 & 3 & 8.4 & 3.4 \\
\hline gi|13928706 & Ncam1 & neural cell adhesion molecule 1 & 2 & 7.9 & 0.7 \\
\hline gi|114052921 & Cadm3 & cell adhesion molecule 3 & 2 & 7.0 & 0.1 \\
\hline gi|9506469 & $\mathrm{Cd} 47$ & Cd47 molecule & 2 & 6.7 & 2.0 \\
\hline gi|9507073 & Nptn & neuroplastin & 3 & 5.8 & 0.2 \\
\hline gi|31543529 & Sirpa & signal-regulatory protein alpha & 2 & 5.3 & 0.6 \\
\hline gi|61557326 & Reep6 & receptor accessory protein 6 & 3 & 4.8 & 0.6 \\
\hline gi|8393864 & Hpcal1 & hippocalcin-like 1 & 3 & 4.3 & 0.1 \\
\hline gi|6981654 & Thy1 & Thy- 1 cell surface antigen & 3 & 3.9 & 0.3 \\
\hline
\end{tabular}

Table 3.5: Identification of synaptic plasma membrane proteins involved in neurite formation and neuronal morphology (GAP43, M6a/b, Thy-1) and adhesion molecules (NCAM, Neurexin, CAM3, neuroplastin, sirpa and CD47).

$\mathrm{N}$-cadherins could not be identified, its intracellular binding partners $\alpha$ - and $\beta$-catenins were present. $\alpha$-catenin directly binds to the filamentous actin, linking the adhesion complex of Cadherin/catenins to the actin cytoskeleton [199].

\subsubsection{Endocytosis related proteins}

Because exocytotic and endocytic sites are suggested to be in close proximity at the plasma membrane, the discovery of proteins belonging to endocytic pathways was not surprising. However, proteins involved in clathrin-mediated endocytosis are not enriched in the docked vesicle fraction, except for clathrin itself (iTRAQ ratio 5.8) 3.6 Components of the AP2complex and endophilin were equally distributed in both fractions. This indicates that the free vesicle fraction is a mixture of the reserve and recycling pool of vesicles. Presumably only uncoated or partially uncoated vesicles could be immunoisolated with an antibody against synaptophysin. This would explain the enrichment of clathrin in the docked vesicle fraction but not in free vesicle immunoisolates. However, a 1:1 ratio for endophilin was surprising, because endophilin is suggested to function at endocytotic retrieval sites, presumably as a membrane 
bending molecule. But in line with our data, a recent study could show that despite acting at the plasma membrane, the majority of endophilin is targeted to the SV pool [200].

Interestingly, proteins of clathrin-independent flotillin endocytotic pathway were also identified. Flotillin 1/2 oligomerizes in distinct membrane microdomains [201], but have been initially described as regeneration molecules in axons of goldfish retinal ganglion cells [202]. Related to this, flotillin microdomains have been reported to associate with Thy-1 (see section adhesion and cell surface molecules) suggesting a role of flotillin/Thy-1 in neurite outgrowth and axon regeneration [203].

\begin{tabular}{|c|c|c|c|c|c|}
\hline \multicolumn{6}{|c|}{ Endocytosis related proteins } \\
\hline GI number & Gene symbol & Name & \# & $\begin{array}{r}\text { iTRAQ } \\
\text { ratio } \\
\end{array}$ & std \\
\hline gi|158636004 & Flot1 & flotillin 1 & 3 & SPM & \\
\hline gi|13929186 & Flot2 & flotillin 2 & 3 & SPM & \\
\hline gi|9506497 & Cltc & clathrin, heavy chain $(\mathrm{Hc})$ & 3 & 5.8 & 1.6 \\
\hline gi|13928818 & Ptprn2 & protein tyrosine phosphatase (Phogrin) & 3 & 2.0 & 0.6 \\
\hline gi|57527421 & Sh3glb2 & endophilin B2 & 3 & 1.4 & 1.0 \\
\hline gi|18034787 & Ap2b1 & adaptor-related protein complex 2 , beta 1 subunit & 3 & 0.8 & 0.2 \\
\hline gi|157823677 & Ap2a1 & adaptor-related protein complex 2 , alpha 1 subunit & 3 & 0.7 & 0.1 \\
\hline gi|162138932 & Ap2a2 & adaptor-related protein complex 2 , alpha 2 subunit & 3 & 0.7 & 0.1 \\
\hline gi|16758938 & Ap2m1 & adaptor-related protein complex 2 , mu 1 subunit & 3 & 0.6 & 0.0 \\
\hline gi|56961624 & Ap2s1 & adaptor-related protein complex 2 , sigma 1 subunit & 3 & 0.6 & 0.1 \\
\hline
\end{tabular}

Table 3.6: Components of the clathrin-dependent and the flotillin-dependent endocytic pathway could be identified, but only flotillins are enriched in the docked vesicle fraction.

\subsubsection{Cytoskeletal and associated proteins}

Cytoskeletal elements are essential for synapse morphology, stabilization of the active zone and vesicular transport in neurons. Also the high morphological plasticity of the synapse requires a dynamic cytoskeleton. Consequently, we identified a wide range of cytoskeletal components, which are known to play a role in the synapse.

Actin and its motor protein myosin were detected. Actin is a major structural component of active zones, where it recruits vesicles to the docking sites and associates with synapsin [125]. Among the identified myosins, myosin 5 interacts with syntaxin $1 \mathrm{a}$ in a $\mathrm{Ca}^{2+}$-dependent manner [204]. Very recently, myosin 5a has been shown to directly associate with Rab3a in its active GTP-bound form, implicating a role in the transport of neuronal vesicles [205]. Another motor protein found in the docked vesicle fraction, myosin 10, has been proposed to have a role 
in neurite outgrowth [206]. Similarly, the microtubule cytoskeleton and specifically the kinesin motor KIF1b is involved in long distance axonal transport of synaptic vesicle precursors [207].

Another component of the presynaptic cytomatrix are septins. Septins are large filamentous proteins and suggested to be part of the filamentous mesh of the active zone that is observed by electron microscopy [55]. They might play a role in positioning SVs at the active zone. In particular septin5, which is detected here, seems to be crucial for the proximity of SVs and active zone elements in the priming/docking stage [208]. Septin5 additionally binds to syntaxin1 in the SNARE complex, competing with SNAP25 [209]. Also septin3 is identified in the docked vesicle fraction, a septin isoform that is exclusively expressed in neurons, enriches in presynaptic terminals and co-localizes with SV markers [210].

Furthermore parts of the spectrin-based membrane skeleton were found. Spectrins and the associated ankyrins interact with with membrane channels and adhesion molecules, including the $\mathrm{Na}^{+} / \mathrm{K}^{+}$ATPase [211], the sodium-calcium exchanger NCX [212], and cadherins [213].

\subsubsection{Signaling molecules}

Signaling at the synapse is very complex and only poorly understood. Therefore, a detailed analysis of the identified signalling proteins was not performed. Nevertheless, many G-proteins (guanine nucleotide binding-proteins) were found in the docked vesicle fraction. G-proteins, especially Gnaq and Gnao, are involved in modulating neurotransmitter release (for review see [214]).

Not surprisingly, also 14-3-3 proteins are present in this fraction as they comprise about $1 \%$ of the total soluble brain proteins. They are involved in many signaling processes, but in the context of synaptic vesicle docking it is worthwhile mentioning that they have been shown to interact with Rim [215] and presynaptic calcium channels [216].

Also CaMKII was detected in our proteomic analysis. CaMKII is a large complex with a well established role in postsynaptic signalling, but is also present in presynaptic terminals [217], associates with vesicles [218] and may have a role in modulating synaptic strength and plasticity. Interestingly, in C. elegans presynaptic CaMKII activates BK channels at the neuromuscular junction [219]. 


\begin{tabular}{|c|c|c|c|c|c|}
\hline \multicolumn{6}{|c|}{ Cytoskeletal and associated proteins } \\
\hline GI number & Gene symbol & Name & $\#$ & $\begin{array}{r}\text { iTRAQ } \\
\text { ratio }\end{array}$ & std \\
\hline gi|109472192 & Dnahc6 & dynein, axonemal, heavy chain 6 & 3 & SPM & \\
\hline gi|109488370 & Dnah2 & dynein, axonemal, heavy chain 2 & 3 & SPM & \\
\hline gi|13591902 & Actn1 & actinin, alpha 1 & 2 & SPM & \\
\hline gi| 29789307 & Kif1b & kinesin family member $1 \mathrm{~B}$ & 2 & SPM & \\
\hline gi|13928704 & Myh10 & myosin, heavy chain 10 , non-muscle & 3 & SPM & \\
\hline gi|6981236 & Myh9 & myosin, heavy chain 9, non-muscle & 3 & SPM & \\
\hline gi|72255527 & Stoml2 & stomatin (Epb7.2)-like 2 & 3 & SPM & \\
\hline gi|109474612 & Rp1 & Oxygen-regulated protein 1 & 2 & SPM & \\
\hline gi|6981696 & Utrn & utrophin & 2 & SPM & \\
\hline gi|157817598 & Invs & inversin & 3 & SPM & \\
\hline gi|109467596 & Ank2 & ankyrin 2, neuronal & 2 & SPM & \\
\hline gi|9507085 & Sept3 & neuronal-specific septin-3 & 2 & SPM & \\
\hline gi|16758016 & Dynll1 & dynein, cytoplasmic, light peptide & 3 & 9.9 & \\
\hline gi|31543764 & Sptan1 & spectrin alpha chain, brain & 3 & 7.3 & 0.0 \\
\hline gi|61557085 & Sptbn1 & spectrin beta chain, brain 1 & 2 & 6.1 & 2.0 \\
\hline gi|13592133 & Actb & actin, beta & 3 & 5.2 & 2.4 \\
\hline gi|13540714 & Plec & plectin 1 & 3 & 5.0 & 1.6 \\
\hline gi|90577179 & Sept5 & septin 5 & 3 & 4.7 & 1.3 \\
\hline gi|157819689 & Sept8 & septin 8 & 3 & 4.5 & 1.3 \\
\hline gi|57164143 & Actr2 & ARP2 actin-related protein 2 homolog & 2 & 4.0 & 1.4 \\
\hline gi|9506371 & Acta1 & actin, alpha 1 , skeletal muscle & 2 & 3.7 & 0.8 \\
\hline gi|166091429 & Sept7 & septin 7 isoform a & 2 & 3.6 & 0.4 \\
\hline gi|109464350 & Kif2 & Kinesin-like protein KIF2 & 2 & 3.3 & 1.2 \\
\hline gi|11560133 & Tuba1a & tubulin, alpha $1 \mathrm{~A}$ & 3 & 3.3 & 3.0 \\
\hline gi|47058982 & Septb & spectrin, beta, erythrocytic & 2 & 3.2 & 3.2 \\
\hline gi|112984124 & Tuba1b & tubulin, alpha 1B & 3 & 3.1 & 2.6 \\
\hline gi|11559935 & Myo5a & myosin Va & 2 & 2.7 & 0.2 \\
\hline gi|158262004 & Tubb4 & tubulin, beta 4 & 3 & 2.1 & 0.9 \\
\hline gi|27465535 & Tubb5 & tubulin, beta 5 & 3 & 2.1 & 1.0 \\
\hline gi|145966774 & Tubb3 & tubulin, beta 3 & 3 & 1.9 & 1.0 \\
\hline gi|55741524 & Tuba4a & tubulin, alpha $4 \mathrm{~A}$ & 2 & 1.7 & 0.8 \\
\hline gi|109495859 & Dnah10 & dynein, axonemal, heavy polypeptide 10 & 3 & 1.2 & 1.0 \\
\hline gi|164698508 & Sept9 & septin 9 isoform 2 & 3 & 1.0 & 0.4 \\
\hline gi|109508026 & Sntb2 & Beta-2-syntrophin (Syntrophin 3) & 2 & 1.0 & 0.2 \\
\hline gi|148491097 & Dync1h1 & cytoplasmic dynein 1 heavy chain 1 & 2 & 0.9 & 0.4 \\
\hline gi|188595680 & Sphkap & A-kinase anchor protein SPHKAP & 2 & 0.9 & 0.1 \\
\hline gi|109480041 & RGD1308350 & similar to hypothetical protein MGC13251 & 3 & 0.7 & 0.1 \\
\hline
\end{tabular}

Table 3.7: Components of the actin and microtubule cytoskeleton, septins and spectrins/ankyrins are present in the docked vesicle fraction. 


\begin{tabular}{|c|c|c|c|c|c|}
\hline \multicolumn{6}{|c|}{ Signaling molecules } \\
\hline GI number & Gene symbol & Name & \# & $\begin{array}{r}\text { iTRAQ } \\
\text { ratio }\end{array}$ & std \\
\hline gi|109475021 & Cdk5rap2 & CDK5 regulatory subunit associated protein 2 & 2 & SPM & \\
\hline gi|13592021 & Pde2a & phosphodiesterase 2A, cGMP-stimulated isoform 2 & 2 & SPM & \\
\hline gi|155369271 & Prkaca & protein kinase, cAMP-dependent, catalytic, alpha & 2 & SPM & \\
\hline gi|8394267 & Shh & sonic hedgehog & 2 & SPM & \\
\hline gi|157822659 & Riok3 & RIO kinase 3 & 2 & SPM & \\
\hline gi|132626321 & Slk & STE20-like kinase & 2 & SPM & \\
\hline gi|6981712 & Ywhaq & $14-3-3$ theta & 2 & SPM & \\
\hline gi|25742825 & $\mathrm{Pi} 4 \mathrm{k}$ & phosphatidylinositol 4-kinase a & 2 & SPM & \\
\hline gi|157818451 & Arl8a & ADP-ribosylation factor-like $8 \mathrm{~A}$ & 2 & SPM & \\
\hline gi|109507443 & Gnal & Guanine nucleotide-binding protein G(olf), alpha & 2 & SPM & \\
\hline gi|13592039 & Rala & v-ral simian leukemia viral oncogene homolog $A$ & 2 & SPM & \\
\hline gi|157821177 & Trio & triple functional domain (PTPRF interacting) & 3 & SPM & \\
\hline gi|109458044 & Nlrp12 & NACHT, LRR and PYD domains-containing protein 12 & 2 & SPM & \\
\hline gi|13591957 & Gnaq & guanine nucleotide binding protein, alpha q & 2 & SPM & \\
\hline gi|9507061 & Pcsk1 & Neuroendocrine convertase 1 & 3 & SPM & \\
\hline gi|9506737 & Gnas & GNAS complex locus gnas1-a & 2 & SPM & \\
\hline gi|157820415 & Rasal1 & RAS protein activator like 1 (GAP1 like) & 3 & 9.0 & 0.8 \\
\hline gi|19424316 & Camkg & calcium/calmodulin-dependent protein kinase $\| \gamma$ & 3 & 8.2 & 1.7 \\
\hline gi|148747524 & Gnb1 & guanine nucleotide-binding protein, beta-1 subunit & 3 & 8.0 & 1.5 \\
\hline gi|108796657 & Camkb & calcium/calmodulin-dependent protein kinase II $\beta$ & 3 & 7.9 & 1.0 \\
\hline gi|29789261 & Gnb2 & guanine nucleotide-binding protein, beta 2 & 2 & 7.5 & 1.7 \\
\hline gi|6978593 & Camka & calcium/calmodulin-dependent protein kinase II $\alpha$ & 3 & 7.3 & 1.6 \\
\hline gi|8394152 & Gnao1 & GTP-binding protein alpha o & 3 & 6.7 & 1.8 \\
\hline gi|6980962 & Gnai1 & guanine nucleotide binding protein $\alpha$ inhibiting 1 & 2 & 6.7 & 0.9 \\
\hline gi|6978595 & Camkd & calcium/calmodulin-dependent protein kinase II $\delta$ & 2 & 6.1 & 2.3 \\
\hline gi|19173774 & Rap2b & RAP2B & 2 & 5.7 & 0.7 \\
\hline gi|62990183 & Ywhaz & 14-3-3 zeta & 2 & 4.9 & 0.4 \\
\hline $\mathrm{gi} \mid 12408298$ & Dpp6 & dipeptidylpeptidase 6 & 2 & 4.5 & 0.2 \\
\hline gi|6981400 & Prkcg & protein kinase $\mathrm{C}$, gamma & 2 & 2.7 & 0.1 \\
\hline gi|109464256 & Cmya5 & Myospyn & 3 & 1.2 & 0.6 \\
\hline gi|42476092 & Gps1 & G protein pathway suppressor 1 & 2 & 1.1 & 0.0 \\
\hline gi|109487963 & Dock2 & dedicator of cyto-kinesis 2 & 3 & 0.8 & 0.2 \\
\hline
\end{tabular}

Table 3.8: Signaling at the active zone includes G-proteins, members of the 14-3-3 family and CaMKII. 


\subsubsection{Unknown proteins}

More than 30 unknown proteins were identified by our analysis of the docked vesicle fraction. These proteins are predicted to be mostly transmembrane proteins and have either an unknown function or localization. Some are indeed novel proteins. Considering the low amount of contaminations and the specificity of the proteins described above, the chance that many of these proteins are in fact associated with the presynaptic membrane is rather high.

\begin{tabular}{|c|c|c|c|c|c|}
\hline \multicolumn{6}{|c|}{$\begin{array}{l}\text { Hypothetical proteins and transmembrane proteins with unknown function or } \\
\text { localisation }\end{array}$} \\
\hline Gl number & Gene symbol & Name & \# & $\begin{array}{r}\text { iTRAQ } \\
\text { ratio }\end{array}$ & std \\
\hline gi|157822793 & Ccdc109a & coiled-coil domain containing 109A & 3 & SPM & \\
\hline gi|56090369 & $\operatorname{Tm} \times 2$ & thioredoxin-related transmembrane protein 2 & 2 & SPM & \\
\hline gi|109484624 & LOC683941 & transmembrane protease, serine 4 & 3 & SPM & \\
\hline gi|62651891 & RGD1309995 & CG13957-PA & 2 & SPM & \\
\hline gi|62660468 & Wdr19 & WD repeat-containing protein 19 & 2 & SPM & \\
\hline gi|62718819 & LOC501488 & rCG41835-like & 2 & SPM & \\
\hline gi|157819311 & RGD1308226 & hypothetical protein LOC296968 & 2 & SPM & \\
\hline gi|56605740 & Wdfy1 & WD repeat and FYVE domain containing 1 & 2 & SPM & \\
\hline gi|109478621 & Setd3 & SET domain containing 3 & 2 & SPM & \\
\hline gi|109492012 & Prp2l1 & proline-rich protein isoform 1 & 2 & SPM & \\
\hline gi|109461608 & LOC687472 & rCG54054-like & 2 & SPM & \\
\hline gi|62078483 & OCIAD1 & OCIA domain containing 1 & 2 & SPM & \\
\hline gi|62079059 & Cend1 & BM88 antigen & 3 & SPM & \\
\hline gi|157822273 & RGD1309188 & hypothetical protein LOC315463 & 3 & SPM & \\
\hline gi|62079015 & RGD1309676 & hypothetical protein LOC361118 & 2 & SPM & \\
\hline gi|157821195 & LOC362419 & hypothetical protein LOC362419 & 2 & SPM & \\
\hline gi|157821401 & LOC683512 & hypothetical protein LOC683512 & 3 & SPM & \\
\hline gi|158262028 & RGD1302996 & hypothetical protein MGC15854 & 3 & SPM & \\
\hline gi|68342019 & Lrrc17 & leucine rich repeat containing 17 & 2 & SPM & \\
\hline gi|109499872 & Fam193a & Hypothetical protein & 2 & SPM & \\
\hline gi|109510841 & ApoO & hypothetical protein Apolipoprotein $\mathrm{O}$ & 3 & SPM & \\
\hline gi|109512114 & RGD1562521 & Similar to Ppnx & 2 & SPM & \\
\hline gi|71361663 & Fam162a & family with sequence similarity 162 , member $A$ & 3 & 8.4 & 5.7 \\
\hline gi|157819829 & RGD1565496 & hypothetical protein LOC300783 & 2 & 4.8 & \\
\hline gi|109481310 & LOC681219 & hypothetical protein LOC681219 & 2 & 4.8 & \\
\hline gi|66730294 & Abhd12 & abhydrolase domain containing 12 & 2 & 4.4 & 2.5 \\
\hline gi|62641302 & RGD1564195 & similar to hypothetical protein & 2 & 3.7 & 0.1 \\
\hline gi|109473862 & LOC686590 & IQ motif and Sec7 domain 1 isoform 2 & 3 & 3.5 & 1.4 \\
\hline gi|51948472 & Tmem30a & transmembrane protein $30 \mathrm{~A}$ & 3 & 2.6 & 0.6 \\
\hline gi|61557143 & Scrn3 & secernin 3 & 2 & 2.2 & \\
\hline gi|62078999 & Traf3ip3 & TRAF3 interacting protein 3 & 2 & 1.3 & \\
\hline gi|67846010 & Rogdi & rogdi homolog & 2 & 1.2 & 0.5 \\
\hline gi|189011652 & Tmprss13 & transmembrane protease, serine 13 & 3 & 1.1 & 0.1 \\
\hline gi|56605828 & Trappc3 & trafficking protein particle complex 3 & 3 & 0.6 & 0.1 \\
\hline gi|109483746 & RGD1307365 & protein QN1 homolog & 3 & 0.5 & 0.2 \\
\hline gi|157786666 & RGD1560058 & hypothetical protein LOC287559 & 3 & 0.5 & 0.3 \\
\hline gi|109464586 & Lrrcc1 & leucine rich repeat and coiled-coil containing 1 & 3 & 0.3 & 0.1 \\
\hline
\end{tabular}

Table 3.9: Proteins identified with unknown function or localization. Some of them are novel, predicted proteins. 


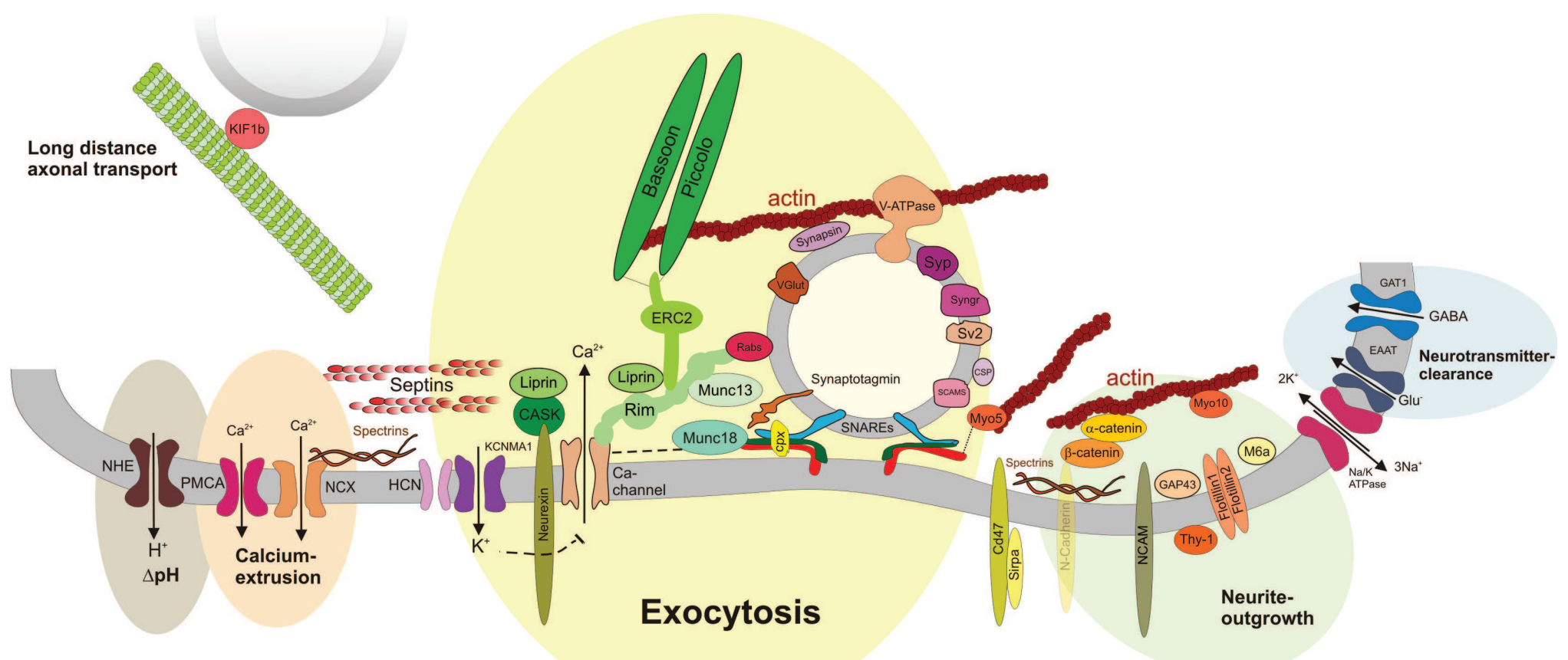

Figure 3.10: Schematic overview of the identified proteins. Proteins were arranged according to their function and interaction partners. Note, that cytoskeletal components were only indicated to avoid overcrowding of the illustration. 


\subsection{Effect of the Rab GTPase modulator GDI on synaptic vesicle docking}

The access to a defined docked vesicle fraction presents an excellent basis to investigate changes in the presynaptic proteome in response to different treatments. We decided to use this fraction to investigate the effect of the Rab effector GDP dissociation inhibitor (GDI) on the organization of the presynapse. Rab GTPases are molecular switches, they cycle between a GTP-bound active state and a GDP-bound inactive state. In the GTP-bound state, Rab proteins are localized to their target membrane, mediating membrane attachment and specifying intracellular membrane fusion reactions. They are bound to synaptic vesicles and have a substantial role in neuronal exocytosis [20]. For instance, Rab3 dissociates from synaptic vesicles after stimulation [149]. A removal of Rab3 from synaptic vesicles can also be induced by GDI. In general, GDI retrieves GDP-bound inactive Rabs from the membrane upon GTP-hydrolysis and forms a soluble cytosolic complex until Rab proteins are actived again and recruited to the membrane [220, 221]. Interestingly, Rab3 proteins can assemble into tripartite complexes with Rim and Munc13 that have been postulated to tether synaptic vesicles to the active zone [68]. Indeed, reduced synaptic vesicle docking is observed in Rab3 mutant mice [222] and C. elegans [79]. In this context, it was examined, whether a GDI-induced extraction of Rab3 from the SV has an effect of vesicle attachment to the plasma membrane or of the protein composition at the active zone.

\subsubsection{Removal of Rab3 from the vesicle membrane does not alter vesicle attach- ment to the plasma membrane}

The GDI dissociation protocol was adapted from [170]. Briefly, samples were incubated for 30 minutes at $37^{\circ} \mathrm{C}$ with purified GDI $[0.5 \mu \mathrm{M}]$ in the presence of excess GDP or the nonhydrolytic GTP analogon GTP $\gamma \mathrm{S}[0.5 \mathrm{mM}]$. GTP $\gamma \mathrm{S}$ locks Rab3 in the active membrane-bound state and thus prevents membrane extraction by GDI. A His-tagged version of GDI from rat species was recombinantly expressed and purified according to [223]. GDI can be introduced at two stages in the established purification procedure (see Fig. 3.11 A ) and both possibilities were examined.

The earliest possible step at which GDI incubation could be applied is after synaptosomal lysis prior to the gradient centrifugation step. Following treatment, the fractions were collected and the migration pattern of docked and free vesicles compared by Western blot. As seen in Fig. 3.11 $\mathrm{B}$, no change in the distribution of the vesicle marker synaptobrevin was observed even though GDI/GDP dependent Rab3 removal was successful. Samples treated with GDI/GDP 
showed two vesicle populations in the same fractions and in the same intensity as untreated samples or samples incubated with GDI/GTP $\gamma$ S or only GDP. Thus, despite the removal of Rab3 from the membrane, GDI incubation appears to have no effect on the attachment of vesicles in this fraction. Clearly, a significant reduction in docked vesicles, as hypothesized, was not seen.

A
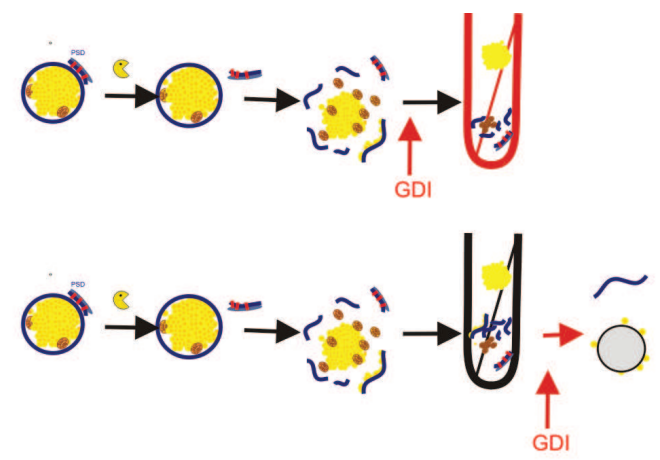

B

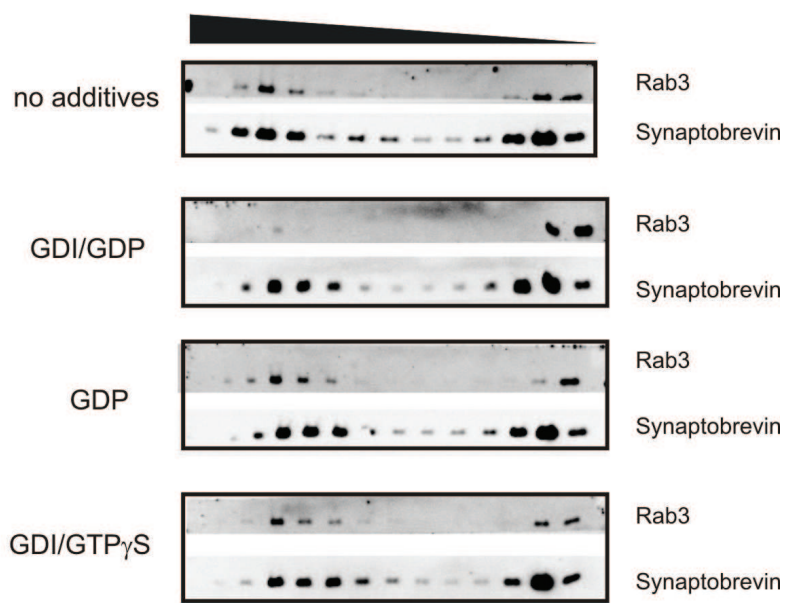

Figure 3.11: (A) Schematic overview of GDI experiments. GDI was added to lysed synaptosomes or directly to the docked vesicle fraction. (B) GDI/GDP, GDI/GTP $\gamma$ S, GDP were added to lysed synaptosomes. Samples were incubated for 30 minutes at $37^{\circ} \mathrm{C}$, followed by a sucrose gradient centrifugation step as in Fig. 3.1 Western blot analysis of gradient fractions showed that Rab3 was removed efficiently from the SPM, but GDI did not alter the migration of docked vesicles.

The next plausible step to add GDI is the docked vesicle fraction. SPM fractions from the gradient were incubated with GDI and GDP, or GTP $\gamma \mathrm{S}$, followed by the immunoisolation of synaptic vesicles. If synaptic vesicles are released from the plasma membrane, the immunoisolates should be devoid of or be reduced in synaptic membrane components. As before, 
Rab3 was efficiently extracted from the membranes, but the immunoisolates still contained the plasma membrane marker $\mathrm{Na}^{+} / \mathrm{K}^{+}$ATPase and components of the active zone (see Fig. 3.12 A). Thus, synaptic membranes appear to remain attached to SVs. Despite the absence of changes when selected proteins were analyzed after GDI treatment, a possibility remains that only a release of few synaptic vesicles has occured that could not be revealed with this method. Few attached vesicle could remain sufficient to immunoisolate the complete docking site.

To investigate smaller changes in vesicle release, we examined vesicle docking by floatation assay and analyzed if a floatation of released synaptic vesicles has occured after treatment with GDI/GDP or GDP. Following GDI incubation, samples were overlaid with an discontinuous sucrose density gradient [SPM, 0.7 M, 0.32 M sucrose] and centrifuged for 3 hours. Fractions were collected and analyzed by Western blotting. The distribution of the material showed no difference between GDI/GDP and GDP treated samples. In both treated and control samples a minor floatation of membranous material was detected at the $0.7 \mathrm{M}$ sucrose/SPM interface. However, these likely do not respond to released synaptic vesicles since parts of the plasma membrane were still present evidenced by the presence of the $\mathrm{Na}^{+} / \mathrm{K}^{+}$ATPase. More likely this membranous material may represent a less dense portion of docked synaptic vesicles (Fig. 3.12B).

This flotation assay has been systematically changed to improve the separation of the material, parameters e.g. different sucrose densities, sample densities or sample conditions were tested. However, neither reducing the sucrose density overlaying the sample nor diluting the sample itself showed any effect in the distribution in the detected signal of synaptic marker proteins (data not shown). Changes in the GDI assay, such as adding salt, pre-incubation with nucleotides or varying incubation time and temperature did not influence the observed vesicle docking, suggesting that under all conditions, synaptic vesicles remain attached to the plasma membrane. Thus, at the stage where vesicle are already docked, Rab3 extraction does not influence the adherence to the membrane. 

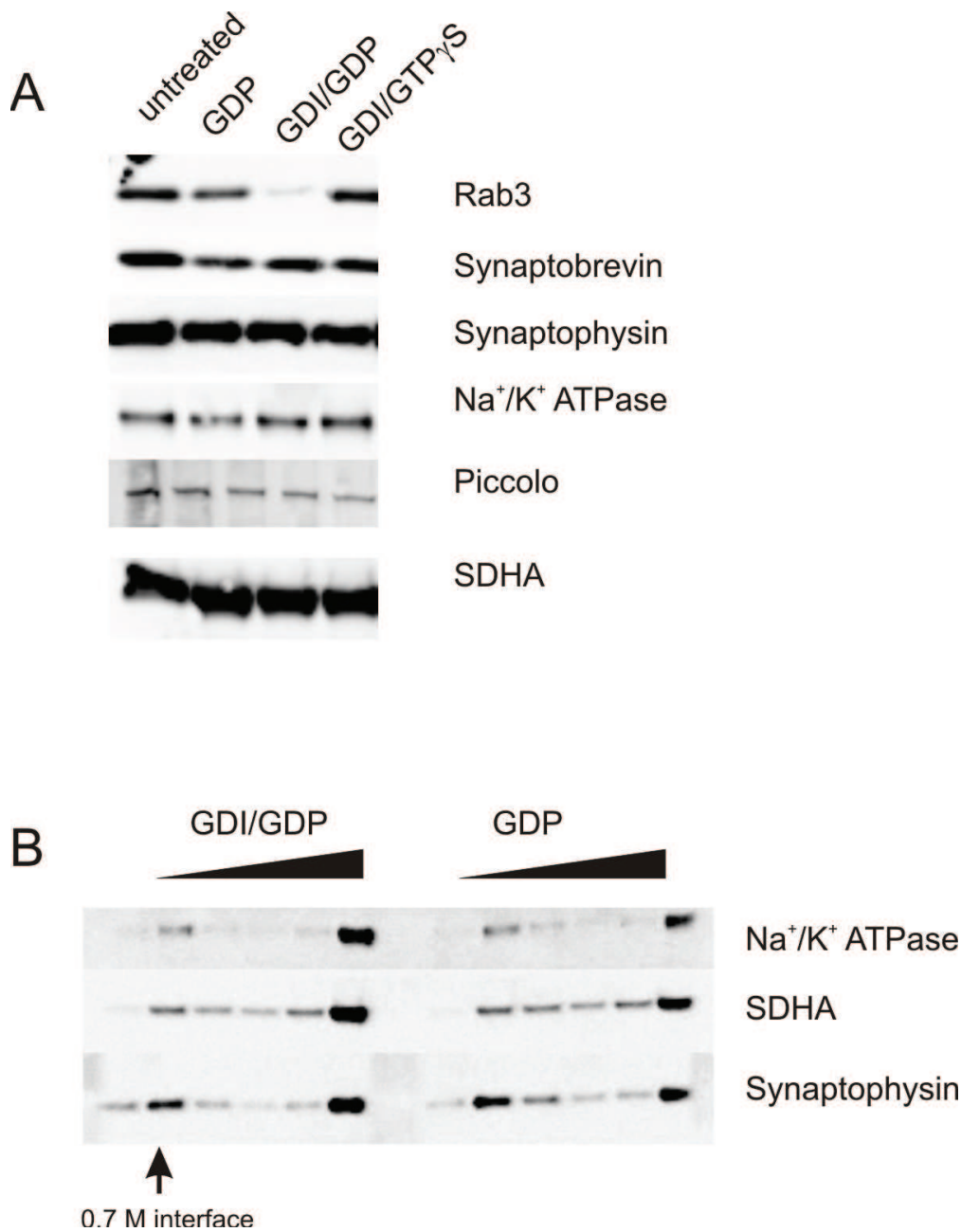

Figure 3.12: (A) Immunoisolation of a docked vesicle fraction after treatment with purified GDI for 30 minutes at $37^{\circ} \mathrm{C}$. Western blot analysis showed that in the GDI/GDP treated docked vesicle fraction, immunoisolated synaptic vesicles are devoid of Rab3 but appear to remain attached to the plasma membrane. (B) Floatation assay of GDI treated docked vesicles. Following GDI treatment, samples were subjected to discontinuous floatation gradient centrifugation. Fractions were collected and analyzed by Western blot. Membranous material was detected at the $0.7 \mathrm{M}$ sucrose interface in all samples, but GDI-dependent vesicle release from the membrane was not observed. 


\subsubsection{GDI treatment does not remodel the protein composition of the active zone}

Rab3 null mutants in D. melanogaster, exhibit defetcs in the distribution of active zone components. This indicated that that Rab3 plays and important role in regulating the protein composition at vesicle release sites [224]. To determine whether removal of Rabs by GDI-treatment also induced a change in already assembled mammalian active zones, we decided to look at changes in the GDI treated samples using iTRAQ analyses. Similar to the previous described comparison of docked and free vesicle (see section 3.2), immunoisolates of untreated, GDI/GDP, GDI/GTP $\gamma$ S and GDP incubated SPM fractions were labeled in parallel, combined, purified, and analyzed. Because direct comparison is limited to two samples within the iTRAQ analysis, all modified samples were compared to the untreated immunoisolates. In order to display enrichment and de-enrichment, a $\log 2$ scale was used in the Y-axes of the graphs to allow changes in ratios to be easily compared. With this, evenly distributed proteins have a value of zero, a two-fold change is illustrated as a value of one and was set as the cut-off value in this experiments.

In line with the previous Western blot analyses, addition of recombinant GDI led to a significant decrease in the amount of Rab proteins detected in the presence of excess GDP. Among the group of Rab proteins, members of the Rab3 family showed the highest reduction as exemplified by $\log 2=1.5$ which is equal to a 3 -fold reduction. As an indication of the specificity of the assay, no changes were observed in the levels of Rab27. Rab27, a recently identified member of the exocytotic Rabs is known to be resistant to GDI-membrane extraction [21]. Strikingly, except for the Rab proteins and GDI, no other component of the docked vesicle fraction was significantly altered (Fig. 3.13 C).

Importantly, treatment with GDP alone or GDI in combination with the inextractable Rab3GTP $\gamma \mathrm{S}$, also did not change the proteome of the docked vesicle fraction. In the GDP alone negative control, none of the over 500 identified proteins were significantly reduced or enriched compared to untreated symaples. Samples treated with GDI/GTP $\gamma \mathrm{S}$ exhibited only an enrichment of GDI among all detected proteins. These data indicate that removal of Rab3 does not remodel the active zone at an assembled stage. More importantly, they also demonstrate that the fraction (or the protocol) can be used to investigate other manipulations on changes in the presynapse. 
A

B
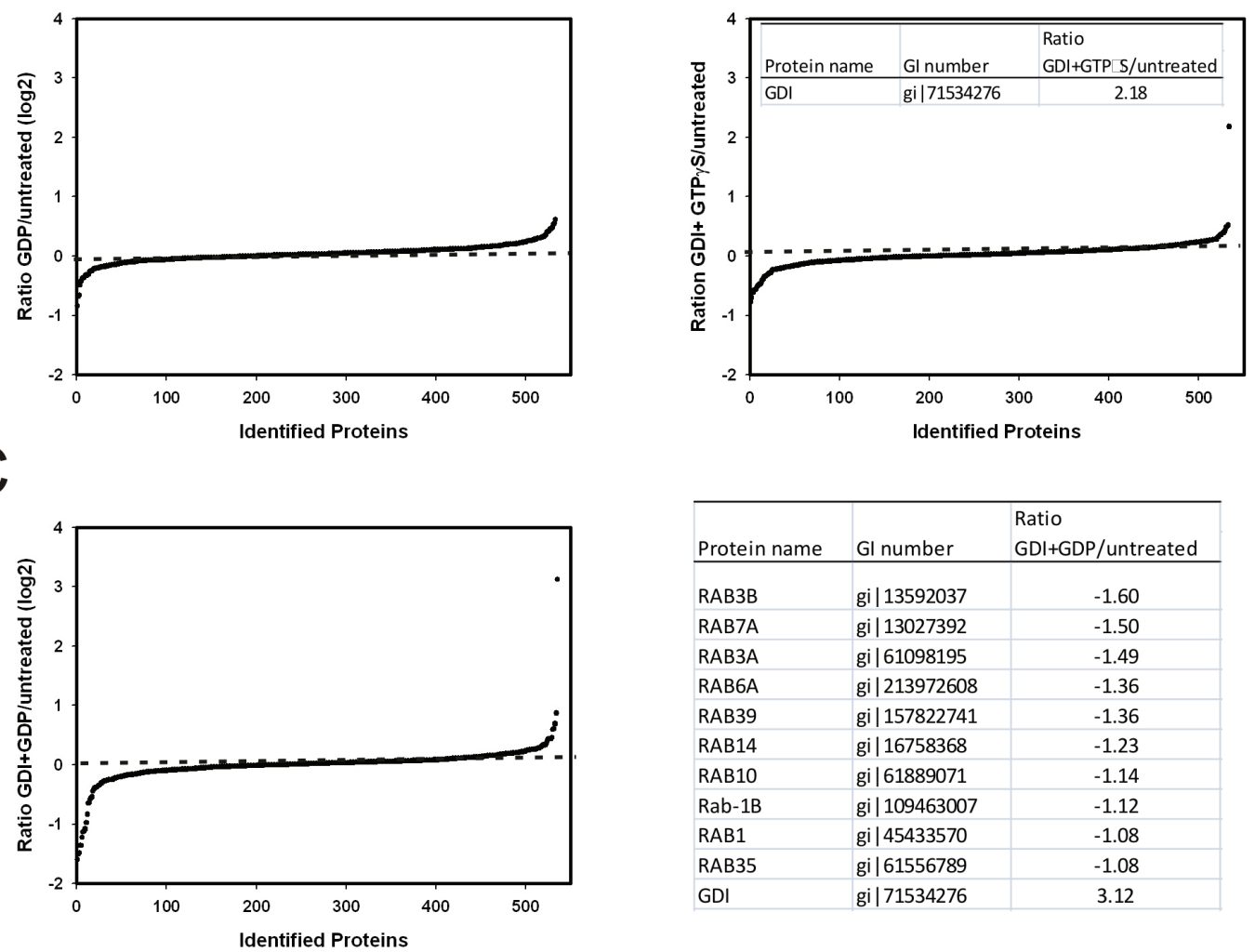

\begin{tabular}{|c|c|c|}
\hline Protein name & Gl number & $\begin{array}{l}\text { Ratio } \\
\text { GDI+GDP/untreated }\end{array}$ \\
\hline RAB3B & gi|13592037 & -1.60 \\
\hline RAB7A & gi| 13027392 & -1.50 \\
\hline RAB3A & gi| 61098195 & -1.49 \\
\hline RAB6A & gi| 213972608 & -1.36 \\
\hline RAB39 & gi| 157822741 & -1.36 \\
\hline RAB14 & gi| 16758368 & -1.23 \\
\hline RAB10 & gi| 61889071 & -1.14 \\
\hline Rab-1B & gi| 109463007 & -1.12 \\
\hline RAB1 & gi| 45433570 & -1.08 \\
\hline RAB35 & gi| 61556789 & -1.08 \\
\hline GDI & gi| 71534276 & 3.12 \\
\hline
\end{tabular}

Figure 3.13: Following incubation with GDI/GDP, GDI/GTP $\gamma$ S or GDP, SPM fractions were immunoisolated and analyzed by ITRAQ analysis as in 3.2 Peptides were labeled with iTRAQ reagents 114-117 and ratios generated as treated/untreated sample. Data are illustrated on a $\log 2$ scale. A value of 0 equals a 1:1 ratio showing that no change had occured. (A) Incubation only with GDP did not result in any changes in the proteome of the docking sites. (B) When retaining Rab3 in its membrane bound state with GTP $\gamma \mathrm{S}$, GDI treatment showed no effect except for an enrichment of GDI itself. (C) GDI/GDP incubation successfully removed Rab proteins, but no other components were affected. The levels of Rab27, which is not extractable by GDI, was unchanged. 


\subsection{JB1 is a novel transmembrane protein localized at presynaptic nerve terminals}

As previous mentioned in 3.2.9 a large number of unknown proteins were present in the docked vesicle fraction. To facilitate selection of the most interesting candidates, proteins were ranked according to their mRNA expression level in the brain, domains composition and extent of evolutionary conservation. Using this strategy, five proteins were chosen for further characterization. Of the five, one of them, JB1, shows much promise as a novel integral member of the presynaptic membrane.

\subsubsection{Identification and characterization of JB1}

JB1 was exclusively found in the SPM (Table 3.9). Officially named hypothetical protein LOC315463 by NCBI, this protein is largely uncharacterized and its function unknown. It is 267 amino acids long and has a predicted molecular weight of 30kDa.

Using the SMART (Simple Modular Architecture Research Tool) program [225, 226], a web-based tool for the study of genetically mobile domains, JB1 was predicted to contain a domain of unknown function (DUF2366) and a transmembrane domain near the N-terminus (Fig. 3.14 A). However, the reliability of the transmembrane domain prediction is uncertain. The transmembrane helix probability was close to the threshold. Additional analyses using other public available computational programs were done to confirm the predicted transmembrane domain. Unfortunately, theses transmembrane prediction analyses yielded conflicting results: The number of identified transmembrane segments varied between 0 (TMHMM [227]), 1 (HMMTOP [228]) and 2 transmembrane helices (DAS [229], TMPRED [www.ch.embnet.org]). A potential transmembrane domain can be supported by sequence coverage of the identified iTRAQ peptides which exclude the predicted transmembrane segment (see Fig. 3.14B). Transmembrane sequences seldom contain the positively charged tryptic cleavage sites and are therefore rarely detected.

Next, sequences homologous to JB1 were identified using BLAST (Basic Local Alignment Search Tool) . The identified sequences were then aligned using the program T-Coffee [230]. As seen in Fig. 3.14 B, JB1 is highly conserved among mammals, but orthologes are also found in D. rerio, D. melanogaster and C. elegans. Even in these model organisms, JB1 function is unknown.

To investigate JB1 transcript distribution in the brain, we made use of the Allen Mouse Brain Atlas, an open interactive, genome-wide image database of gene expression [231]. The online available data are obtained by RNA in situ hybridization of tissues derived from 8-week 
old male mouse brains and are integrated into a detailed digital reference atlas. As shown in Fig. 3.14C, JB 1 transcripts were present in the brain with a particularly high level of expression in the hippocampus.

To enable further characterization of JB1, a rabbit polyclonal antibody was generated against recombinant full length JB1 protein. In immunoblots, anti-JB1 antibody recognised a single $30 \mathrm{kDa}$ protein band in crude synaptosomes in excellent agreement with the predicted size of JB1 (Fig. 3.15A). Lysates of HEK293 cells overexpressing a JB1-GFP fusion protein additionally confirmed the specificity of the anti-JB1 antibody. Immunoblots with anti-JB1 and anti-GFP antibody both recognized the fusion protein at approximately $55 \mathrm{kDa}$. Lysates of untransfected HEK293 cells did not show chemiluminescence indicating that these cells don not express endogenous JB1 (Fig. 3.15B).

\subsubsection{JB1 is a transmembrane synaptic protein}

To determine if JB1 indeed contains a transmembrane region, we used a panel of detergents to analyze the behavior of endogenous JB1 following detergent extraction. JB1 was completely extractable from crude synaptosomal membranes by the non-ionic detergent Triton-X 100, partially soluble with zwitterionic detergent CHAPS and insoluble when treated with alkaline $\mathrm{Na}_{2} \mathrm{CO}_{3}$. Alkaline $\mathrm{Na}_{2} \mathrm{CO}_{3}$ releases peripheral membrane proteins by converting the membrane into flat sheets [232]. The solubility pattern of JB1 resembled the single transmembrane domain-containing SNARE syntaxin1A (Fig. 3.15 C). In contrast, the multispanning transmembrane receptor NR1 remained insoluble in all conditions. Thus, these studies indicate that JB1 indeed contains a bona fide transmembrane domain.

To determine the expression profile of JB1, multiple tissues were dissected from a 6-week old male wistar rat, homogenized and membrane fractions enriched using a Triton-X-114 phase partitioning assay [166]. The highest amounts of JB1 were observed in brain tissues (cortex, cerebellum, spinal cord), supporting that the main function of this protein might be neuronal (Fig. 3.15 D). JB1 is also present in heart and to a lesser extent in kidney and pancreas. Interestingly JB1 was absent in skeletal muscle tissue, although both cardiac and skeletal muscle coordinate excitation-contraction coupling. In the pancreas, the antibody detected 3 protein bands at $30 \mathrm{kDa}, 35 \mathrm{kDa}$, and a major band at $50 \mathrm{kDa}$, respectively. The larger polypeptides might indicate the existence of different splice variants in pancreas. To exclude detection biases due to sample preparation, tissues were additionally probed for the ubiquitously expressed mitochondrial protein SDHA as well as the Golgi SNARE syntaxin6. These proteins were detected in all tissues. 
A Hypothetical protein LOC315463/ RGD1309188
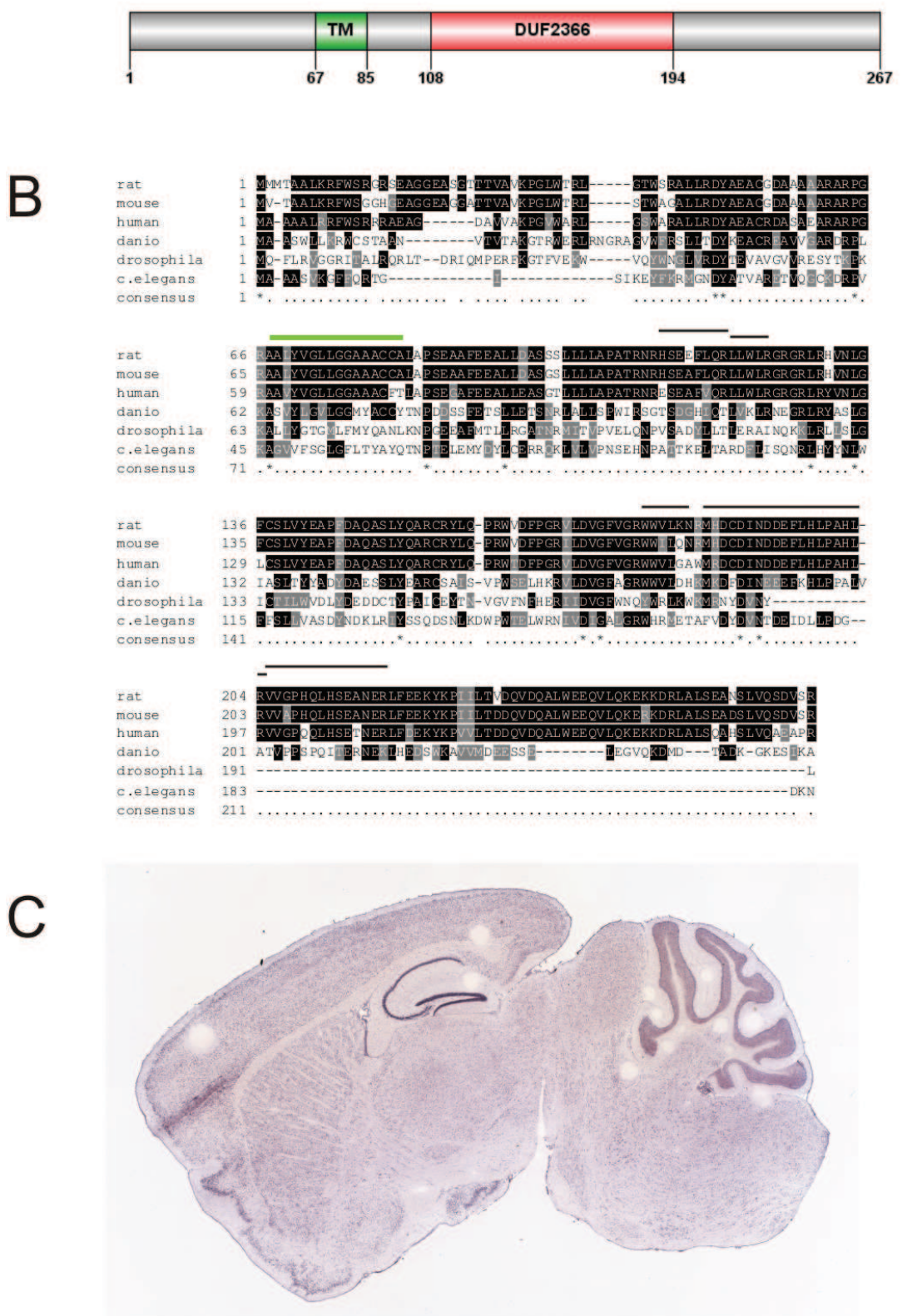

Figure 3.14: (A) Hypothetical protein LOC315463 (JB1) has one potential transmembrane domain and a domain of unknown function (DUF2366). Data were obtained using the online tool SMART. (B) Amino acid sequences of JB1. Sequences were deduced from sequence analysis of rat, mouse, human, zebrafish, fly and worm. Black lines denote peptides identified by iTRAQ. The green line highlights the putative TM domain. (C) JB1 transcript distribution in mouse brain. Strongest expression was observed in the hippocampal CA3 region and in the dentate gyrus. Data were obtained from the Allen Mouse Brain Atlas (accesion number NM178619). 
Next the subcellular distribution of JB1 was analyzed by Western blotting. JB1 is strongly enriched in the synaptosomal fraction P2' and the presynaptic plasma membrane fraction LP1. Importantly, it is completely absent from synaptic vesicles (see Fig $3.15 \mathrm{E}$ ). Because in this experiment synaptosomes were not protease treated, the LP1 fraction still contained parts of the postsynaptic density exemplified by the immunoreactivity of PSD95. PSD95 exhibited a strong co-enrichment with JB1. The SV protein synaptobrevin is diminished in the LP1 fraction and highly enriched in the synaptic vesicle fraction. These data agree with the iTRAQ result of JB1 being only present in the docked vesicle fraction and essentially identifies JB1 as a novel synaptic protein. However, attention should be paid to a remaining possibility of JB1 being a postsynaptic component. Although the low postsynaptic contamination detected in the iTRAQ data point to a presynaptic role of JB1, the employed subfractional analysis is not sufficient to completely exclude a postsynaptic localization.

\subsubsection{JB1 is associated with presynaptic structures}

To exclude the possibility that JB1 is a postsynaptic contaminant, the developed validation assay for the removel of postsynaptic components (see section 3.1.1.2) was applied to verify the indicated presynaptic localization of JB1. Trypsinized and untreated synaptosomes were immunofluorescently labeled with a combination of antibodies against JB1 and synaptotagmin or JB1 and PSD95 and the distribution of the detected signals analyzed by confocal microscopy. As shown in Fig 3.16 A, signals observed for JB1 (green) and synaptotagmin (red) co-localize extensively in untreated synaptosomes. Linescans drawn across the puncta showed overlapping signal intensity peaks for both proteins. This co-localization did not change in trypsinized samples. Neither the signal intensity nor the number of puncta observed for JB1 was affected as is observed for synaptotagmin. In contrast to this, in the samples co-stained with PSD95, the extent of co-localization significantly decreased in trypsinized synaptosomes. Moreover, the number of PSD95 puncta was greatly diminished compared to untreated synaptosomes while the amount of observed JB1 puncta appear to stay constant. Linescans verified that the remaining JB1 puncta were devoid of any postsynaptic signal.

The immunofluorescent images represented only a small fraction of the total population of synaptosomes. To analyze the total protein amount in the sample, the same fractions were additionally compared by Western blotting (Fug. 3.16 B). As expected, JB1 is not degraded by protease treatment.The detected signal was comparable to untreated synaptosomes, whereas PSD95 levels was reduced significantly upon trypsin digestion.

Finally, we compared the endogenous distribution pattern of JB1 in rat hippocampal neurons with synaptotagmin and PSD95. Hippocampal neurons were fixed at 14 DIV and immuno- 

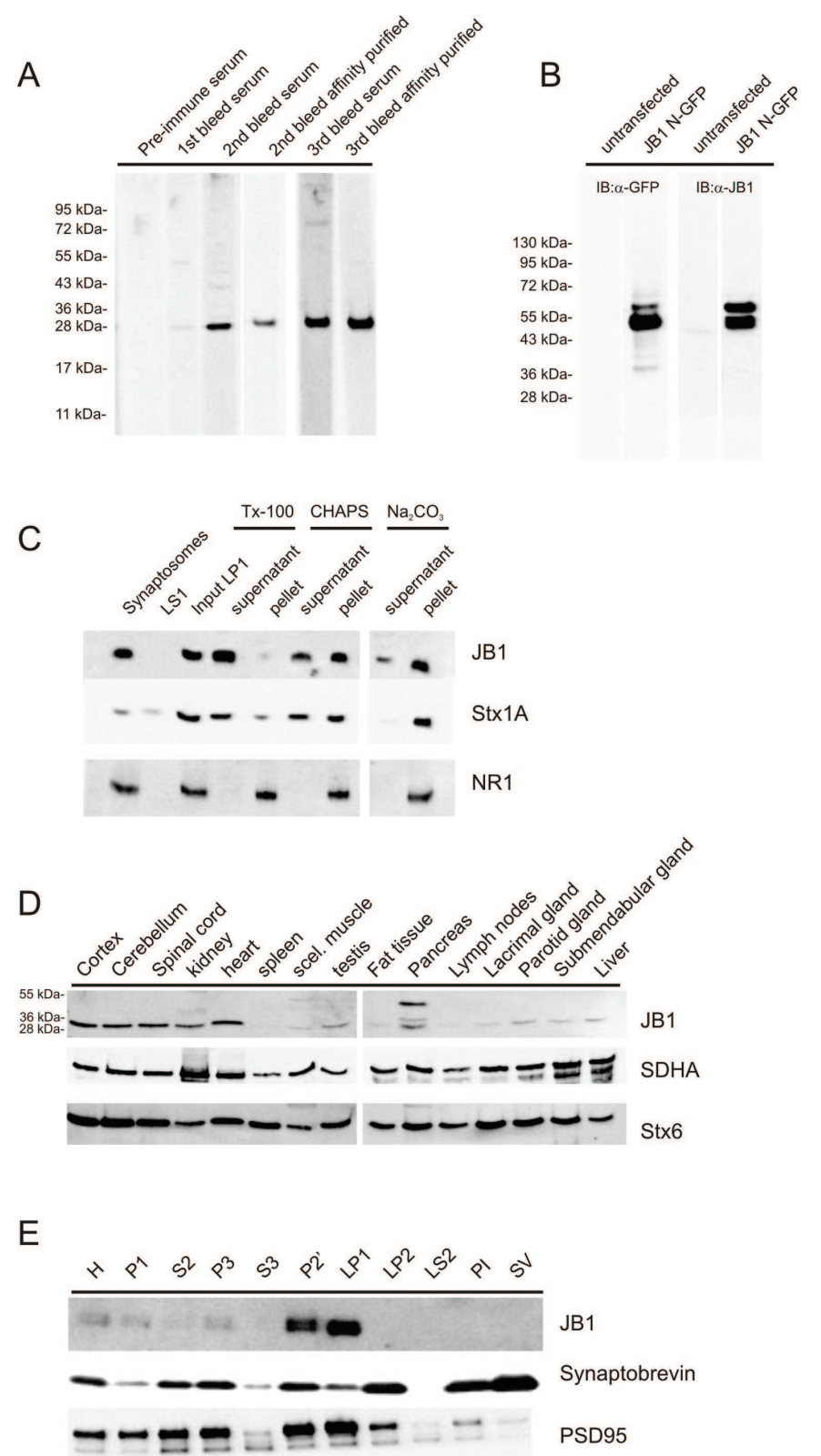

Figure 3.15: (A) Characterization of antiseren showed that JB1 is present in synaptosomes. $22 \mu \mathrm{g}$ synaptosomes per lane were resolved by SDS-PAGE and transferred to a nitrocellulose membrane. Different batches of serum (1:2000) or affinity purified (1:500) anti-JB1 rabbit polyclonal antibody were tested for their efficacy to recognise endogenous JB1. A single protein band corresponding to the predicted molecular weight was detected since the second bleeding. (B) Immunoblot of untransfected and transfected HEK293 lysates overexpressing a JB1-GFP fusion protein. HEK293 cells were transiently transfected and harvested after $24 \mathrm{~h}$. JB1-GFP was detected with antiGFP antibody (left) and anti-JB1 antibody (right). (C) Solubility of JB1 from pelleted synaptic plasma membrane fractions (LP1). LP1 pellets were resuspended in 1\% Tx-100, 1\% CHAPS or 100mM sodium carbonate $\mathrm{pH} 11.4$ and incubated for 30 minutes at $4{ }^{\circ} \mathrm{C}$. The remaining insoluble parts were re-pelleted for 30 minutes at 100000 g. Solubility of JB1 resembled that of syntaxin 1. (D) Expression of JB1 in different tissues. $1 \mathrm{mg}$ of each tissue homogenate was subjected to membrane extraction with Triton-X-114 and equal volumes loaded per lane. (E) Western blot analysis of subcellular fractions from the rat brain. $10 \mu \mathrm{g}$ of homogenate $(\mathrm{H})$, nuclear and large membrane pellet (P1), crude brain cytosol and small organelles (S2), small cell organelles (P3), brain cytosol (S3), crude synaptosomes (P2'), presynaptic plasma membrane (LP1), crude synaptic vesicles (LP2), synaptic cytosol (LS2), (P1) and pure synaptic vesicles (SV) was loaded and probed for JB1, a postsynaptic marker JB1 and the SV protein synaptobrevin. JB1 is strongly enriched in synaptosomes and presynaptic membrane fraction. 
A
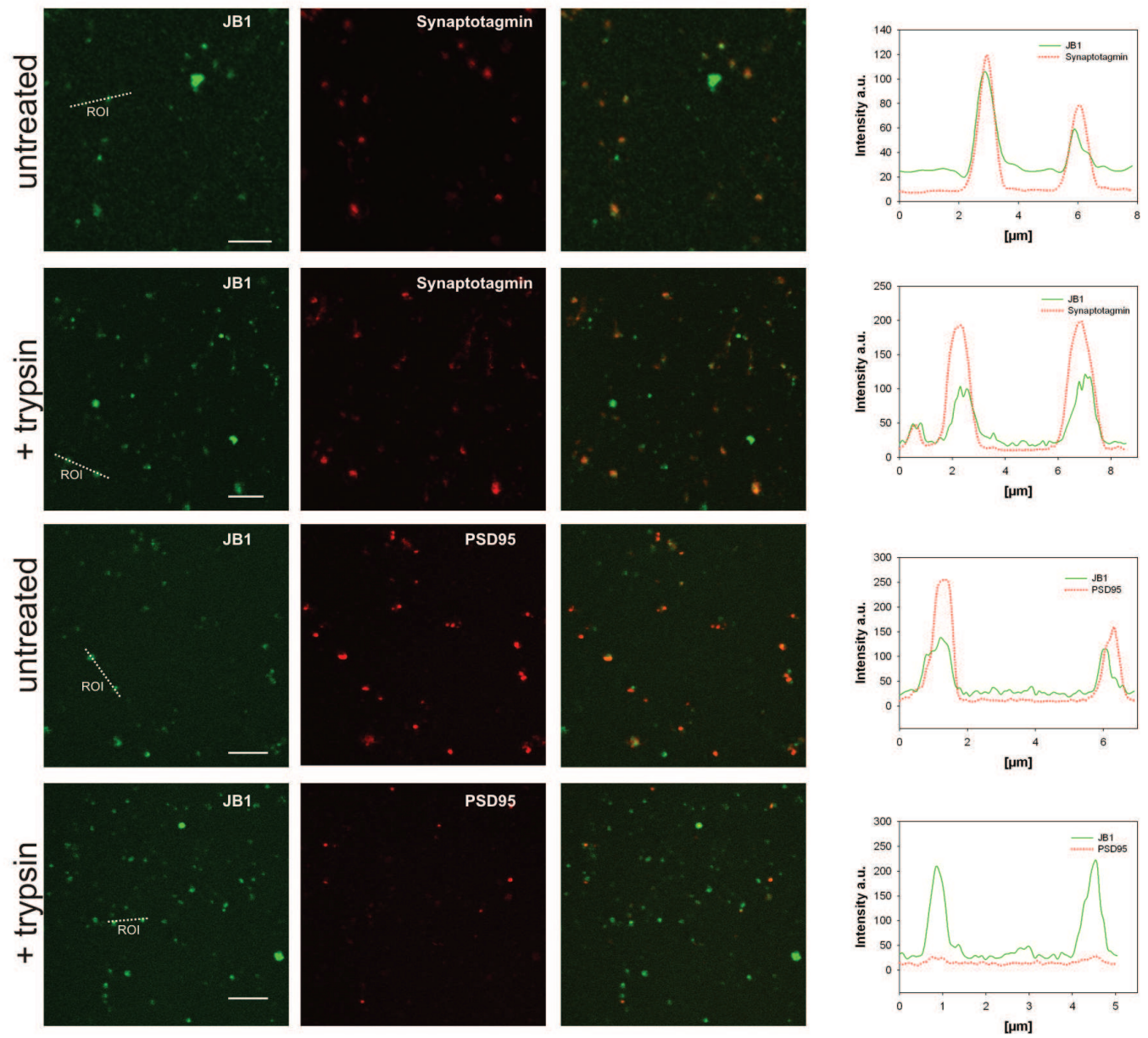

B

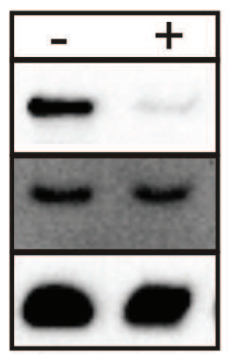

trypsin
PSD95

JB1

\section{Synaptobrevin}

Figure 3.16: (A) Immunofluorescent staining of synaptosomes. Protease treated and untreated synaptosomes prepared as in 3.1.1.2 were co-labeled with antibodies directed against JB1 (green) and synaptotagmin (red) or JB1 and PSD95. JB1 puncta were resistant to protease treatment. (B) Western blot analysis of the total protein in the fraction confirmed that JB1 remains present in shaved synaptosomes, whereas levels of PSD95 are depleted. 
labeled with antibodies against JB1 and synaptotagmin or PSD95. As seen in Fig. 3.17 JB1 labeling showed a punctate staining in neuronal processes. A closer examination of synaptic nerve terminals revealed that these puncta partially overlapped with the synaptic vesicle protein synaptotagmin (see arrowheads). In contrast to this, no overlap was detected with the dendritic protein PSD95. Moreover, JB1 puncta appeared to be localized juxtaposed to the postsynaptic density (see arrowheads).

In summary, the accumulated data agree with the iTRAQ results and demostrates that JB1 is a bona fide novel presynaptic protein. 

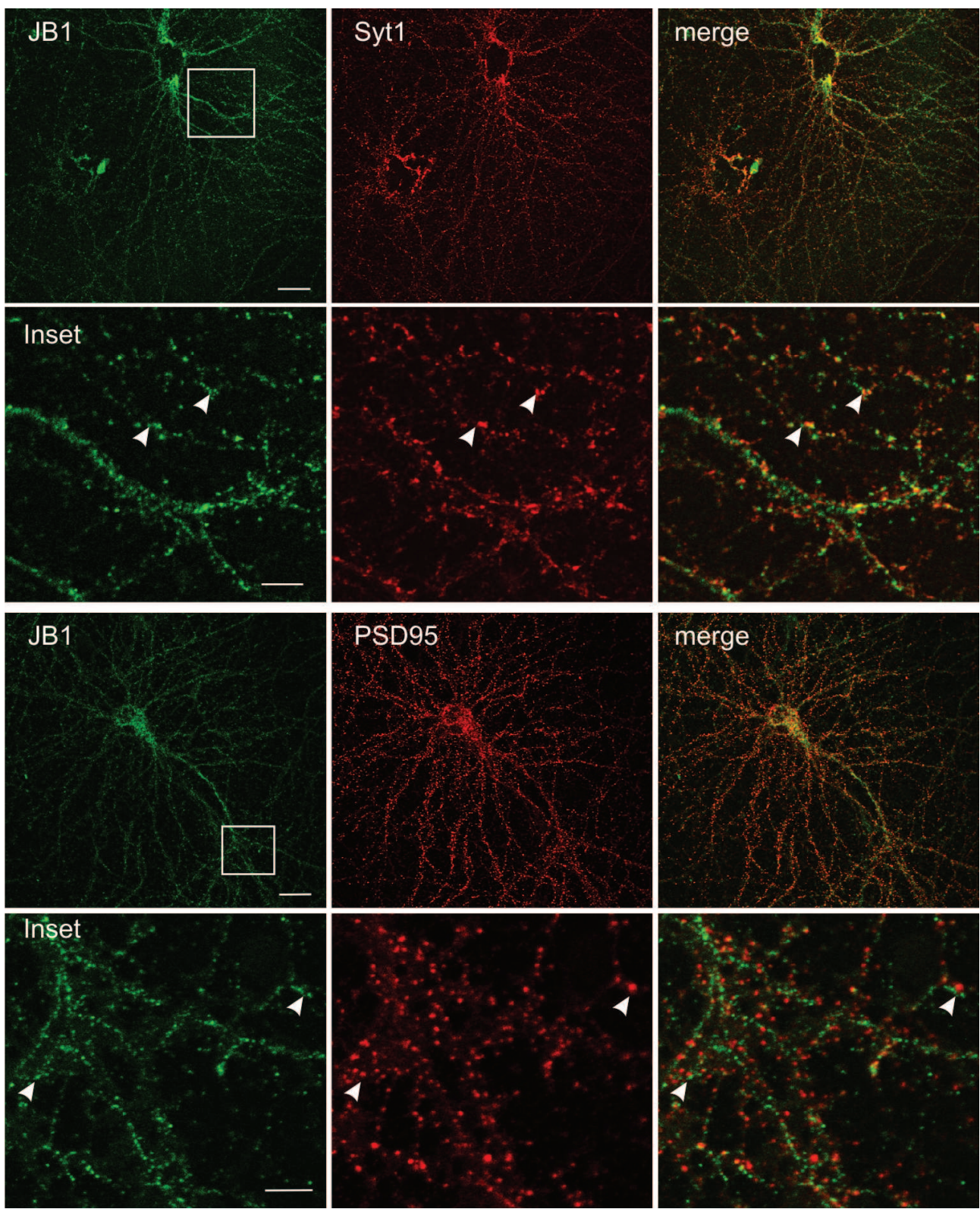

Figure 3.17: Hippocampal neurons DIV14 were double labeled with antibodies against JB1 and synaptotagmin or JB1 and PSD95. Immunostaining for JB1 (green) was observed in neuronal processes. Magnified views (boxed) revealed a punctate staining for JB1 with a partial colocalization with the synaptic vesicle protein synatotagmin in contrast to the postsynaptic protein PSD95. Arrowheads highlight co-labeled structures. 


\section{Discussion}

\subsection{A novel protocol to separate pre- and postsynaptic compart- ments}

As outlined in the introduction, excitatory synapses are characterized by their asymmetric organization with a presynaptic nerve terminal containing synaptic vesicles and the presynaptic machinery, a synaptic cleft, and a postsynaptic signaling complex called the postsynaptic density (PSD). Postsynaptic densities can be isolated from synaptosomes by non-ionic detergents that solubilize the presynaptic, but not the postsynaptic specialization [172]. These PSD fractions have been characterized extensively by proteomic approaches, revealing a large number of proteins that are bona fide postsynaptic proteins $([233,133,135,234])$. However, these preparations are not exclusively postsynaptic as they also contain a number of presynaptic proteins exemplified by the discovery of bassoon [97].

On the other side, the isolation of presynaptic specializations has been addressed for years, but compared to the large number of postsynaptic density constituents, comparatively few presynaptic components have been identified. Core active zone proteins have been mainly found by low-throughput approaches such as yeast-two-hybrid screens: Rim [78], ERCs 1 and 2 [88], CASK [117] and Mint [120]. These systems are very useful in the detection of pairwise protein interactions, but cannot reveal the global constituents of the presynaptic nerve terminal. Thus, it is commonly believed that the molecular composition of the presynaptic site is incompletely characterized. The identification of presynaptic protein components is mainly limited by difficulties in obtaining a sufficiently active zone-enriched fraction that is devoid of postsynaptic contamination. Unfortunately, plasma membrane fractions isolated by density gradient centrifugation still exhibit intact pre- and postsynaptic adhesion [172]. Consequently, no satisfactory and comprehensive characterization of the presynaptic proteome was previously available, although a number of proteomic studies have been performed to profile synaptic protein constituents: 
- In 2001 and more comprehensively in 2005, Phillips and co-workers reported a detergent based isolation of a presynaptic particle fraction [152, 151]. This protocol is based on a sequential $\mathrm{pH}$-dependent Triton-X-100 extraction of synaptosomes. A first extraction with $1 \%$ Triton-X-100 in Tris $\mathrm{pH} 6.0$ solubilizes the plasma membrane but paired preand postsynaptic structures remain in the insoluble pellet. A second extraction step with an elevated $\mathrm{pH} 8.0$ solubilizes only the presynaptic network that is collected and repelleted after a dialysis back to $\mathrm{pH}$ 6.0. This was the first attempt to separate the presynapticfrom the postsynaptic compartment, allowing the identification of proteins from an isolated presynaptic fraction. Characterizing both fractions using MudPIT, they could show that the presynaptic proteins dynamin, clathrin heavy chain, syntaxin1, SNAP25 and Munc18 are present in the presynaptic particle fraction, but controversially, bassoon, piccolo, Rim and synaptotagmin were exclusively found in the postsynaptic fraction. Other known active zone components, e.g. Munc13, ERC2 or Liprins could not be identified.

- A different approach to obtain the presynaptic fraction, that did not address a separation of pre- and postsynaptic compartments, but included an affinity purification step of a docked vesicle fraction was developed by Morciano and co-workers in 2005 and 2009 [173, 153]. Briefly, synaptosomes were osmotically lysed, synaptic plasma membranes fractionated by gradient centrifugation an immunoisolated with an antibody specific for the synaptic vesicle protein SV2. The analyzed immunoisolates contained synaptic vesicle proteins, cytoskeletal elements, active zone molecules, plasma membrane components, mitochondrial proteins and metabolic enzymes. However, active zone proteins were mostly identified by additional Western blotting and some major components such as Liprins, CASK and ERC2 remain undetected. Notably, an examination for PSD contamination was omitted.

- Using the protocol developed by Philipps and co-workes, Abul-Husn et al. systematically approached the presynaptic proteome by generating a comprehensive list of presynaptic proteins [235] by including known protein interactions derived from literature mining and combining them with their own data, the data from Phillips et al. [151], and Morciano et al [173]. The final "presynaptic core list" of 117 proteins in total included only proteins identified two or more times in their MS analyses and contained largely synaptic vesicle proteins, plasma membrane components and cytoskeletal elements. Strikingly, active zone proteins are completely absent from this list.

Here I have established a novel protocol that permits the immunoisolation of a presynaptic compartment from proteolytically "shaved" synaptosomes. From this fraction I was able to 
generate the first global protein composition of presynaptic docking sites using quantitative mass spectrometry. Using this strategy, I was able to identify novel components of the presynapse and characterize one of them in detail. Additionally, I showed that proteolytically treated synaptosomes/immunoisolated presynaptic AZ fractions can be used to study global changes in their proteome following external manipulations. In the following, the new procedure described in this work will be compared to the existing studies in relation to sample preparation and analysis, and data quantity and quality.

\subsubsection{Method}

Two fundamental problems currently limit the characterization of the presynaptic proteome: Sample complexity and the dynamic range of the analytes. Due to the large number of proteins and the heterogeneity, proteins in low abundance have a high chance of being obscured by those of high abundance. Also highly hydrophobic proteins such as channels and transporters remain challenging, because these proteins cannot be properly solubilized and are consequently difficult to identify.

By removing the postsynaptic density (whicht itself contains several hundred proteins [133, [135, 234]) is not only important to ascertain presynaptic localization of the identified proteins, but is also the most effective step to reduce the complexity of the sample. This strategy has already been adressed by two groups, but a satisfactory separation of pre- and postsynaptic compartments could not be achieved so far:

- The detergent based protocol reported by Philipps and coworkers depends on differential solubilities of the proteins in the sample to achieve separation of both synaptic compartments [152]. However, transmembrane proteins and also scaffolding proteins like bassoon, piccolo and Rim have a tendency to be difficult to extract and thus could explain their existence in the detergent resistent postsynaptic pellet [236].

- A detergent-independent approach using a denaturing protocol was reported by Berninghausen et al. By combining urea and DTT to disassemble the connection of preand postsynaptic membranes, they enriched bassoon and piccolo in a presynaptic membrane fraction together with syntaxin, SNAP25 and synaptotagmin while postsynaptic preoteins like NR1, GluR1 and PSD95 are diminished. They could provide a calculated 3.2-fold enrichment, but did not achieve homogeneity of the pre-and postsynaptic membranes [237].

In this study the postsynaptic membrane was almost quantitatively removed from synaptosomes after a protease treatment step. Western blotting and immunofluorescence microscopy con- 
firmed that trans- and postsynaptic components such as Neuroligin, NR1, GluR1 and PSD95 were successfully removed while presynaptic components and synaptic vesicle proteins remained intact. I further combined this depletion strategy with an enrichment of presynaptic proteins (affinity purification of a docked vesicle fraction). A separation with such a high degree of efficiency has not been achieved before.

However, the novel protocol developed in this study is based on the proteolytic cleavage of the synaptic cleft. Unlike a detergent based strategy, using proteases entails the risk of unwanted protein degradation in the presynaptic compartment. Therefore, proteolytic conditions were carefully tested and optimized to minimize presynaptic protein degradation, but still provide sufficient proteolytic activity to cleave the trans-synaptic connections. Importantly, trypsin activity could be immediately stopped at the desired time by adding an irreversible serine protease inhibitor. Furthermore, one need to keep in mind that the elevated temperature incubation at $30^{\circ} \mathrm{C}$ might cause an increase of endogenous protease reactivity.

In contrast to the other separation strategies, the extracellular domains of presynaptic transmembrane proteins and adhesion molecules are removed resulting in a potential loss of function of these proteins. Therefore studies of these proteins are limited with this method. Additionally, synaptic adhesion molecules have been proposed to have a role in the organization and modulation of synaptic structure ([238, 239, 240]). Thus, a possible effect on protein stability induced by the removal of these proteins cannot be completely excluded. However, the large number and identity of the detected proteins did not support a change in the protein composition as a consequence of protease usage. Even intracellular proteins prone for degradation like the $500 \mathrm{kDa}$ protein piccolo or synapsin did not show major degradation after protease treatment. This indicates that the presynaptic proteins inside of the membrane remain preserved.

Despite the successful removal of the postsynaptic density and a subsequent immunoisolation step, the presynaptic docked vesicle fraction remains a biological complex sample. Sample complexity often exceeds the capability of mass spectrometers although a continuous improvement in accuracy and throughput has taken place. This not only leads to a loss in information, it can also produce sample bias towards proteins with high abundance. In comparison to other proteomic analyses [153], we additionally employed an upstream peptide fractionation by strong cation exchange chromatography (SCX) prior to LC-MS/MS analysis. Such a fractionation increases resolution and minimize ion suppression effects by separating tryptic peptides according to charge and hydrophobicity prior to the MS analysis. When analyzing samples with large amounts and a diverse heterogeneity of proteins, this method has succeeded conventional 1D SDS-PAGE protein separation [241], which is often limited by an unavoidable band overlap due to the large protein amounts. Conventional 2D-gel electrophoresis, separating in the first dimension by isoelectric focusing point (IEF) and in the second dimension by mass, 
has major problems resolving hydrophobic proteins [242] and is therefore limited for the use of membrane proteins [243]. An alternative gel-based 2D-electrophoretic separation method named BAC/SDS-PAGE has been applied by Morciano et al. [173]. This separation improves the resolution of membrane proteins [244, 245], but might be biased against hydrophilic proteins.

The SCX fractionation method has already been proven to be a good choice for synaptic plasma membrane preparations [246]. By doing a comprehensive study on the mass spectrometric side of the analysis of crude synaptic plasma membranes, this group showed that a comparable amount of sequencing information could be obtained from each SCX fraction as to the one-dimensional analysis of whole digest sample. This improvement allowed for an identification of a large number of proteins that have been underrepresented in other studies, in particular active zone components and transmembrane channels [151, 153]. Another advantage of a SCX purification is its ability to remove excess labeling chemicals and other substrates that possibly interfere with MS analysis.

Finally, unlike previous proteomic studies of the presynapse, I have applied a quantitative mass spectrometric strategy by using the stable isotope labeling reagent iTRAQ. A relative quantitation comparing the immunoisolates from the docked vesicle fraction and free vesicles was done to enrich for presynaptic proteins and distinguish SV proteins.

\subsubsection{Presynaptic proteome}

What constitutes the proteome of presynaptic preparations? Clearly, presynaptic signature molecules and organelles such as synaptic vesicles, active zone components and presynaptic channels have to be present. For the first time, with this novel protocol, we could identify almost all active zone proteins and a large number of transmembrane channels by mass spectrometric analysis. In addition, synaptic vesicle proteins (SCAMPs, SV2s and neurotransmitter transporters) that remained unidentified in the presynaptic particle fraction [152, 151], or were only detected by Western blotting [153], were identified and quantified in this preparation, providing a comprehensive list of SV proteins.

A good presynaptic proteome needs to be additionally devoid of postsynaptic contamination. As a result of the successful removal of the postsynaptic density, the final immunoisolates only exhibit three remaining proteins exclusively localized to the postsynaptic density among 500 identified proteins. Interestingly, in the immunoisolated docked vesicles of synaptosomes that have not been addressed for PSD removal as done by [173, 153], PSD components were also not detected by mass spectrometry. But unfortunately, the authors did not additionally 
test for postsynaptic contamination as it has been done for the undetected presynaptic proteins [153].

In this study we have performed the first correlation profiling of presynaptic proteins contained in the docked synaptic vesicle and free synaptic vesicle fractions using iTRAQ quantification. Originally established as a label-free approach to characterize the human centrosome [139] or map whole organelles [247], protein correlation profiling simplifies the analysis of complex samples that can only be enriched by fractionation but not completely purified to homogeneity. In our study, this strategy was used to discriminate genuine presynaptic proteins from synaptic vesicle constituents. It assumes that proteins which are contained in the synaptic vesicle would have the same degree of enrichment, whereas proteins involved in attaching the SV to the plasma membrane or residing in the presynaptic membrane are thought to have a different degree of enrichment. Indeed, all detected proteins that have been reported to be localized to synaptic vesicles [138] were additionally confirmed as such by a non-enrichment in the iTRAQ ratio.

However, some contaminants may behave biochemically similar and therefore may copurify with the docked vesicle fraction. An example could be the persisting detection of mitochondria in this study. However, this hypothesis remains debatable and mitochodondria might also represent integral components of the active zone. Although the immunofluorescent images of the docked vesicle fraction did not show significant overlap between mitochondria and the active zone protein piccolo, other studies suggest a direct attachment of this organelle to nerve terminals via syntaphilin [248, 249]. Syntaphilin was not detected in this study, but a possible association at close sites at the plasma membrane but not directly at the active zone cannot be excluded. In fact, electron tomography showed evidence for a cytoskeletal structure that connects mitochondria to the presynaptic membrane near active zones [250]. This supports the idea that mitochondrial localization at active zones is essential to regulate the calcium concentration and metabolic demand of synaptic transmission. At the neuromuscular junction of D. melanogaster, an additional role for mitochondria in the assembly of the actin cytoskeleton within presynaptic boutons [251] and mobilization of synaptic vesicle from the reserve pool to the readily releasable pool [252] has been proposed.

In conclusion, the identification and quantification of the presynaptic signature molecules together with a low detection of contaminants proves a high quality for this presynaptic preparation. As a consequence, the remaining proteins that were identified raised in interest. In this study we could identify 506 proteins in the docked vesicle fraction. After a subtraction of 217 mitochondrial localized proteins, 289 proteins remain associated to the presynaptic compartment. Strikingly, compared to the existing presynaptic proteomic studies, the amount of detected proteins has doubled [153] or tripled [151]. A graphical description and comparison 


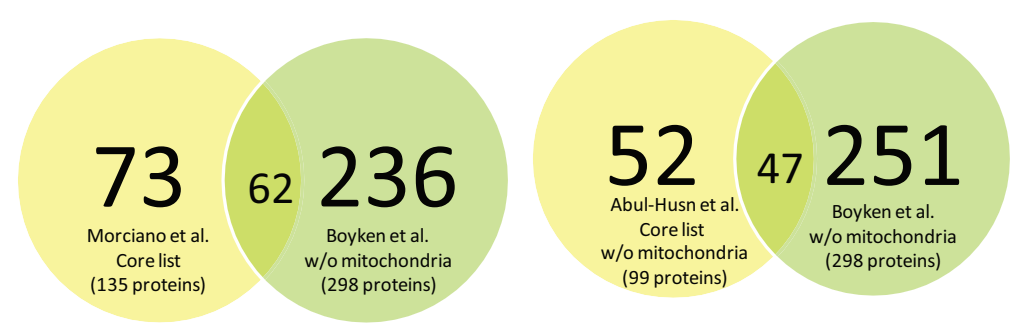

Figure 4.1: A significant overlap between the dataset of Morciano et al., Abul-Husn et al. and the data obtained in this work was observed. Mitochondrial proteins are substracted from all data.

of the data obtained by the different proteomic approaches [153, 235] is illustrated in Fig. 4.1, An overlap between proteins that were identified in this study was observed. This overlapping proteins include synaptic vesicle proteins, high abundant plasma membrane molecules and cytoskeletal components. A complete list of proteins detected in both approaches can be found in the appendix.

Among the proteins that were identified by Morciano et al. but were not detected in this study are dynamin $1 / 2$, SNAP $(\alpha, \beta, \gamma)$, several Rab proteins (1b, 11, 3D, 5A, 6B, 7A), GTPbinding proteins (GNA11, GNAI2, GNAZ, GNG3), additional isoforms of 14-3-3 (eta and epsilon) and the plasma membrane calcium ATPase (PMCA3 and PMCA4). Their data also include other adhesion molecules (NCAM2, N-CAM L1, contactin-1, hepaCAM) and cytoskeletal components (actin and tubulin). Also the glial protein MBP was found. Similarly, the presynaptic core list generated by Abul-Husn et al. contained more endocytic related proteins such as dynamin, clathrin light chain and synaptojanin. This list also included additional signaling proteins (calmodulin, MAPK, LYN, protein phosphatase $2 \mathrm{~B}$, phospholipase $\mathrm{C}$ ), other adhesion molecules (L1CAM, contactin 1) and cytoskeletal components (MAP2, actinin 2/3, tropomyosin, tubulin 6) that were not identified in this study. This list also contained the glial protein MBP.

What are these 298 proteins? For the first time, this study presents a global view on the molecular architecture of the presynapse. The data proves evidence that similar to the PSD, neurotransmitter releasing sites exhibit a number of functionally different protein groups that all together form a highly organized network to ensure precise synaptic transmission. Aside from the proteins responsible for regulating exocytosis, this includes a large number of cytoskelatal elements and proteins involved in the mechanisms of calcium extrusion and neurotransmitter clearance as well as neurite outgrowth. Many of these proteins have already been reported to be localized to synaptosomes or synaptic plasma membrane fractions by other nonproteomic approaches. For example, the plasma membrane $\mathrm{Ca}^{2+}$ - ATPase [186] or EAAT1 and EAAT2 [191] were detected in synaptosomes by Western Blotting. However, these prepa- 
rations have contained pre- and postsynaptic compartments. With this study we can not only additionally validate the presence of these proteins by mass spectrometry, we can further prove their localization to the presynaptic membrane. However, a dual existence of these proteins on both sides of the synaptic cleft cannot be excluded, because the postsynaptic membrane was removed in this approach. Although the number of identified proteins could be increased to almost 300 (500 with mitochondria), some known presynaptic proteins remain underrepresented. For example presynaptic receptors such as kainate [253, 254] and Eph [255], were not identified in this study. This lack of identification may have been caused by the tryptic cleavage of molecules exposed to the synaptic cleft, but it also implies that the presynaptic proteome generated in this study, although being very comprehensive, remains incomplete.

\subsubsection{Identification of novel proteins}

Coming back to the belief that the knowledge of the presynaptic proteome is limited, for many proteins the detection at presynaptic sites was a novelty. Some of these proteins are already characterized but have not been assigned to a presynaptic function, making them interesting candidates that are worthwhile to be investigated in more detail. Strikingly, also more than 30 novel (predicted) proteins could be identified and enriched among the presynaptic proteins. Considering the quality of the preparation, these novel molecules have a high chance of being true bona fide presynaptic constituents. One of these novel proteins, termed JB1, was further characterized and shown to be localized to presynaptic membranes. This makes it a novel presynaptic protein of unknown function. JB1 shows no resemblance to other neuronal proteins and apart from a potential transmembrane region does not contain any known domains. Thus hypothesizing a function in synaptic transmission is difficult without additional studies. Nevertheless, JB1 is conserved and RNAi interference in C.elegans is embryonic lethal, suggesting this protein plays an important role. A specific expression in excitatory tissues such as brain and heart indicates a role in excitatory processes. Interestingly, although expressed in cardiac muscle, JB1 could not be detected in skeletal muscle. In contrast to skeletal muscle, cardiac muscles require extracellular calcium for normal excitation-contraction to occur. But implicating a calcium dependent role for JB1 only based on this is pure speculation and needs to be further elucidated. A continuation of the characterization will hopefully reveal its synaptic contribution.

\subsubsection{Versatility/usage of the method}

The methods developed so far have been mainly used to identify and localize synaptic proteins. A broader application of these protocols in combination with other techniques is limited. 
The use of detergents limits many methods. For proteomic applications, there are three major disadvantages: First, a complete solubilization of all proteins contained in the sample cannot be guaranteed. The proteomic data obtained by [237] is indicative of this limitation. Second, all detergents interfere with mass spectrometry to some extent when they are present at high concentrations. Triton-X-100 has a low critical micellar concentration (CMC) [256] and thus cannot be removed by dialysis. This also effects the identification of protein complexes, because other detergents can only be used in addition, but not as an alternative. The Phillips protocol [152] is additionally based on a Tris-buffered system. Thus, quantitative labeling strategies that involve reactive amine groups (such as iTRAQ) cannot be applied. Furthermore many functions of membrane proteins can only be studied in a lipid bilayer, which is perturbed or lost in detergents. Also spectophotometric monitoring can be ineligibly to some extent, because detergents such as Triton-X-100 strongly absorb UV light.

Urea, as used in the denaturing protocol [237], is a chaotropic reagent that denatures proteins at high concentrations (4-8 M). Even at low concentrations (1 M) it can perturb protein structure and alter protein function [257]. Due to the heterogeneity of the proteins in synaptosomes, the probability that the stability of some proteins is affected is rather high. In addition urea causes extensive changes in the behavior of the solvent environment that in turn weakens or disrupts native protein complexes. Repetitive freeze/thaw cycles additionally affects protein stability.

The protocol developed in this study provides the broadest application range. The use of trypsin as a protease does not interfere with subsequent mass spectrometric applications. The protocol is also compatible with immunofluroscence microscopy. Additionally, molecular complexes are retained and can be isolated at a later stage by subsequent immunoisolation strategies. But most important, proteins remain active within the synaptosomal membrane and can be functionally addressed. This, in combination with the applied iTRAQ quantification, allows to study proteomic changes and protein interactions by targeting specific proteins with effectors that promote or inhibit its function.

\subsection{Investigation of proteomewide changes in synaptic vesicle dock- ing site upon treatment with effectors}

An example of this application is shown by the removal of Rab proteins from synaptic vesicles by GDI. A hypothesis that Rab3 plays an important role in synaptic vesicle recruitment rather than docking has emerged over the years [258]. Although Rab3 have a suggested role in regulating dense core vesicle docking [16, 15], the precise function of Rab3 in synaptic trans- 
mission is still not fully understood. Rab3 directly interacts with the active zone scaffolding protein Rim [75] providing a physical link between synaptic vesicles and the presynaptic active zone [68]. Additionally, a similar reduction of docked vesicles in Rab3-, Rim- or Rab3/Rimdouble mutants in C. elegans were observed, assuming that this interaction might be crucial for the tethering of synaptic vesicles [79]. On the other hand, a recent study showed that Rab3 directly associates with the tail of the actin motor protein myosin 5a. supporting a role of Rab3 in the transport of neuronal vesicles rather than the attachment to the plasma membrane [205]. However, as outlined in the introduction 1.1.1, docking is mainly defined as a morphological observation of vesicles located in close proximity to the plasma membrane. This only provides a static picture at a given time point and does not reflect the dynamic actions of docked vesicles that constantly undergo association and dissociation from the plasma membrane [259]. This is further supported by the relative high amount of synaptic vesicles observed in the docked vesicle fraction compared to the free vesicle fraction. Such a high number of vesicles does not reflect the morphologically observed low number of vesicles attached to the plasma membrane.

Here, alterations in the docked vesicle fraction were analyzed as a consequence of a GDI induced removal of Rab proteins from synaptic vesicles. The effect of GDI treatment on the attachment of SVs as well as global changes in the presynaptic proteome as a consequence of GDI incubation were examined using the iTRAQ based quantitation. In this study, addition of recombinant GDI only led to a significant decrease in the amount of Rab proteins in the presence of excess GDP, but synaptic vesicles remained attached to the plasma membrane. This provides direct evidence that Rab3 is not required for attaching synaptic vesicles to the plasma membrane after docking had taken place. However, docking might not be determined by a single protein-protein interaction. In fact, deletion of a large number of proteins (Munc18, Munc13, Rim [260], synaptotagmin [47], syntaxin [2]) result in changes in the amount of vesicles attached to the plasma membrane, indicating that docking is possibly mediated by a series of protein-protein interactions. Thus, Rab3 might only contribute to docking as a transient contact between vesicle and active zone, that is followed by additional factors that determine the attachment of vesicles to the plasma membrane.

- Munc18 has been shown to promote docking in vivo [4, 7, 6], possibly mediated by binding to the "closed" conformation of synatxin 1 [8, 9]. But at this stage it remains elusive if the attached vesicles are only docked or already primed. Therefore, it is also possible that Munc18 binds to the "open" synatxin 1, stabilizing the SNARE acceptor complex [261] and thus facilitating SNARE assembly [262, 263]. On the vesicular side synaptotagmin seems to be the prime candidate for docking aside from its function as the neuronal calcium sensor. In chromaffin cells, synaptotagmin has been shown bind 
to the SNARE acceptor complex, anchoring vesicles and promoting SNARE assembly [261, 47]. However, docking mechanisms in chromaffin cells might be different from neurons, as these cells do not contain many of the active zone proteins.

- The SNARE complex is very stable [264]. Assuming that SNARE proteins are already assembled at this stage (possibly as a consequence of Munc18), this interaction itself might be sufficient to keep vesicles attached.

- Cytoskeletal components, in particular the F-actin network, might entrap synaptic vesicles in the subplasmalemmal cytoskeleton. Actin dynamics play an important role in in the presynaptic nerve terminal [265, 266] and interact with the vesicle protein synapsin [125]. Considering the large number of cytoskeletal components identified in this study, I assume that the cytoskeletal network persists in the isolated docked vesicle fraction. Apart from a possible direct interaction between the cytoskeleton and synaptic vesicle, de-attachment of vesicles might just simply be abolished because these whole organelles get physically entangled in these network.

- It cannot be completely ruled out that the Rab3 mediated effect on docking is sufficiently compensated by Rab27b. These Rab proteins share common GEFs [267, 268] and appear to have overlapping functions in synaptic vesicle exocytosis [21]. Similar to Rab3, Rab27 has also been shown to influence docking [17, 18], but in contrast to Rab3, Rab27 is resistent to GDI-membrane retrieval [21]. Instead, inactive GDP-bound Rab27b has been suggested to persist on membrane as an inactive homodimer [269].

Strikingly, except for the reduction of Rab proteins, the molecular composition of the active zone remains constant. Precisely, the amount of more than 500 proteins that were identified were unchanged. Surprisingly, this included the Rab3 interacting protein Rim. Rim proteins are large multi-domain molecules that are proposed to function as central organizers interacting with multiple proteins [87, 67, 85]. However, the Rab3-binding site is localized to the $\mathrm{N}$-terminus, whereas other parts of Rim mediate different functions. For example, the central PDZ domain interacts with calcium channels, localizing them to the active zone [84]. Importantly, these domains appear to act autonomously[270]. Thus, a disruption of the Rab3-Riminteraction does not interfere with Rim function in tethering calcium channels to release sites. This possibly accounts for Rim localization at the active zone. Taken together I hypothesize that Rab3 is possibly involved in the first contact of synaptic vesicles with the presynaptic active zone via its interaction with Rim and thus might initiate the attachment of vesicles to the plasma membrane, but it does not restrict the diffusion of these vesicles after docking. 


\subsection{Conclusion and outlook}

Proteomic analysis of subcellular compartments have a tremendous potential to help understanding complex processes such as synaptic transmission. Addressing the emerging need to comprehensively profile the presynaptic proteome, this thesis describes the establishment and use of a new proteolytic protocol that can immunoisolate active zones including a step that efficiently removes the postsynaptic density from synaptosomes. This study identified more than 500 proteins in a presynaptic docked vesicle fraction. In addition to the entire active zone, synaptic vesicle constituents, previously reported synaptic proteins, molecules with a hitherto unidentified synaptic function and novel proteins were detected. This work also uncovered a novel presynaptic protein, JB1. However, its precise function remains to be elucidated. Ongoing knock-down and over-expression studies in D. melanogaster and rat hippocampal neurons will hopefully shed some light on its neuronal function. Nevertheless, the gathered presynaptic proteome only represents an average molecular composition of all synapse types in cortical and hippocampal neurons and cannot distinguish between excitatory and inhibitory synapses.

The established protocol allowed to measure quantitative changes in the protein composition at these synapses. As an example, I analyzed the docked vesicle proteome downstream of Rab3 removal by GDI treatment. Our results prove evidence that Rab3 alone does not mediate this attachment. Moreover, Rab3 removal after SV docking does not change the protein composition of active zones components, suggesting that the main role of Rab3 role is targeting synaptic vesicles to the active zone. The feasibility of this protocol provides a powerful basis to further dissect molecular interactions and mechanisms at the presynaptic active zone. For example, a quantitative comparison of immunoisolated docked vesicles from glutamatergic excitatory and GABAergic inhibitory synapses would significantly account for the understanding of the molecular heterogeneity of central synapses. 


\section{References}

[1] J. J. Chua, S. Kindler, J. Boyken, and R. Jahn, "The architecture of an excitatory synapse," J Cell Sci, vol. 123, no. Pt 6, pp. 819-23, 2010. 1

[2] M. Hammarlund, M. T. Palfreyman, S. Watanabe, S. Olsen, and E. M. Jorgensen, "Open syntaxin docks synaptic vesicles," PLoS Biol, vol. 5, no. 8, p. e198, 2007. 2, 3, 82

[3] T. Schikorski and C. F. Stevens, "Morphological correlates of functionally defined synaptic vesicle populations," Nat Neurosci, vol. 4, no. 4, pp. 391-5, 2001. 2

[4] T. Voets, R. F. Toonen, E. C. Brian, H. de Wit, T. Moser, J. Rettig, T. C. Sudhof, E. Neher, and M. Verhage, "Munc18-1 promotes large dense-core vesicle docking," Neuron, vol. 31, no. 4, pp. 581-91, 2001. 2, 82

[5] R. F. Toonen, O. Kochubey, H. de Wit, A. Gulyas-Kovacs, B. Konijnenburg, J. B. Sorensen, J. Klingauf, and M. Verhage, "Dissecting docking and tethering of secretory vesicles at the target membrane,” EMBO J, vol. 25, no. 16, pp. 3725-37, 2006. 2

[6] R. F. Toonen, K. Wierda, M. S. Sons, H. de Wit, L. N. Cornelisse, A. Brussaard, J. J. Plomp, and M. Verhage, "Munc18-1 expression levels control synapse recovery by regulating readily releasable pool size," Proc Natl Acad Sci U S A, vol. 103, no. 48, pp. 18332-7, 2006. 2,82

[7] R. M. Weimer, J. E. Richmond, W. S. Davis, G. Hadwiger, M. L. Nonet, and E. M. Jorgensen, "Defects in synaptic vesicle docking in unc-18 mutants," Nat Neurosci, vol. 6, no. 10, pp. 1023-30, 2003. 2, 82

[8] H. de Wit, L. N. Cornelisse, R. F. Toonen, and M. Verhage, "Docking of secretory vesicles is syntaxin dependent," PLoS One, vol. 1, p. e126, 2006. 2, 3, 82

[9] A. Gulyas-Kovacs, H. de Wit, I. Milosevic, O. Kochubey, R. Toonen, J. Klingauf, M. Verhage, and J. B. Sorensen, "Munc18-1: sequential interactions with the fusion machinery stimulate vesicle docking and priming," J Neurosci, vol. 27, no. 32, pp. 8676-86, 2007. 2, 82

[10] H. de Wit, A. M. Walter, I. Milosevic, A. Gulyas-Kovacs, D. Riedel, J. B. Sorensen, and M. Verhage, "Synaptotagmin-1 docks secretory vesicles to syntaxin-1/snap-25 acceptor complexes," Cell, vol. 138, no. 5, pp. 935-46, 2009. 2 
[11] E. Chieregatti, J. W. Witkin, and G. Baldini, "Snap-25 and synaptotagmin 1 function in ca2+-dependent reversible docking of granules to the plasma membrane," Traffic, vol. 3, no. 7, pp. 496-511, 2002. 2

[12] N. E. Reist, J. Buchanan, J. Li, A. DiAntonio, E. M. Buxton, and T. L. Schwarz, "Morphologically docked synaptic vesicles are reduced in synaptotagmin mutants of drosophila," J Neurosci, vol. 18, no. 19, pp. 7662-73, 1998. 2

[13] E. M. Jorgensen, E. Hartwieg, K. Schuske, M. L. Nonet, Y. Jin, and H. R. Horvitz, "Defective recycling of synaptic vesicles in synaptotagmin mutants of caenorhabditis elegans," Nature, vol. 378, no. 6553, pp. 196-9, 1995. 2

[14] M. Fukuda, J. E. Moreira, V. Liu, M. Sugimori, K. Mikoshiba, and R. R. Llinas, "Role of the conserved whxl motif in the $\mathrm{c}$ terminus of synaptotagmin in synaptic vesicle docking," Proc Natl Acad Sci U S A, vol. 97, no. 26, pp. 14715-9, 2000. 2

[15] J. R. van Weering, R. F. Toonen, and M. Verhage, "The role of rab3a in secretory vesicle docking requires association/dissociation of guanidine phosphates and munc18-1," PLoS One, vol. 2, no. 7, p. e616, 2007. 2, 81

[16] A. M. Martelli, G. Baldini, G. Tabellini, D. Koticha, and R. Bareggi, "Rab3a and rab3d control the total granule number and the fraction of granules docked at the plasma membrane in pc12 cells," Traffic, vol. 1, no. 12, pp. 976-86, 2000. 2, 81

[17] H. Gomi, K. Mori, S. Itohara, and T. Izumi, "Rab27b is expressed in a wide range of exocytic cells and involved in the delivery of secretory granules near the plasma membrane," Mol Biol Cell, vol. 18, no. 11, pp. 4377-86, 2007. 2, 83

[18] K. Kasai, M. Ohara-Imaizumi, N. Takahashi, S. Mizutani, S. Zhao, T. Kikuta, H. Kasai, S. Nagamatsu, H. Gomi, and T. Izumi, "Rab27a mediates the tight docking of insulin granules onto the plasma membrane during glucose stimulation," J Clin Invest, vol. 115, no. 2, pp. 388-96, 2005. 2,83

[19] M. Geppert, V. Y. Bolshakov, S. A. Siegelbaum, K. Takei, P. De Camilli, R. E. Hammer, and T. C. Sudhof, "The role of rab3a in neurotransmitter release," Nature, vol. 369, no. 6480 , pp. 493-7, 1994. 2

[20] O. M. Schluter, F. Schmitz, R. Jahn, C. Rosenmund, and T. C. Sudhof, "A complete genetic analysis of neuronal rab3 function," J Neurosci, vol. 24, no. 29, pp. 6629-37, 2004. 2, 59 
[21] N. J. Pavlos, M. Gronborg, D. Riedel, J. J. Chua, J. Boyken, T. H. Kloepper, H. Urlaub, S. O. Rizzoli, and R. Jahn, "Quantitative analysis of synaptic vesicle rabs uncovers distinct yet overlapping roles for rab3a and rab27b in ca2+-triggered exocytosis," J Neurosci, vol. 30, no. 40, pp. 13441-53, 2010. 2, 46, 63, 83

[22] E. A. Neale, L. M. Bowers, M. Jia, K. E. Bateman, and L. C. Williamson, "Botulinum neurotoxin a blocks synaptic vesicle exocytosis but not endocytosis at the nerve terminal," J Cell Biol, vol. 147, no. 6, pp. 1249-60, 1999. 2

[23] L. von Ruden and E. Neher, "A ca-dependent early step in the release of catecholamines from adrenal chromaffin cells," Science, vol. 262, no. 5136, pp. 1061-5, 1993. 3

[24] T. Voets, E. Neher, and T. Moser, "Mechanisms underlying phasic and sustained secretion in chromaffin cells from mouse adrenal slices," Neuron, vol. 23, no. 3, pp. 607-15, 1999. 3

[25] U. Becherer and J. Rettig, "Vesicle pools, docking, priming, and release," Cell Tissue Res, vol. 326, no. 2, pp. 393-407, 2006. 3

[26] H. Plattner, A. R. Artalejo, and E. Neher, "Ultrastructural organization of bovine chromaffin cell cortex-analysis by cryofixation and morphometry of aspects pertinent to exocytosis," J Cell Biol, vol. 139, no. 7, pp. 1709-17, 1997. 3

[27] K. Broadie, A. Prokop, H. J. Bellen, C. J. O’Kane, K. L. Schulze, and S. T. Sweeney, "Syntaxin and synaptobrevin function downstream of vesicle docking in drosophila," Neuron, vol. 15, no. 3, pp. 663-73, 1995. 3

[28] J. B. Sorensen, K. Wiederhold, E. M. Muller, I. Milosevic, G. Nagy, B. L. de Groot, H. Grubmuller, and D. Fasshauer, "Sequential n- to c-terminal snare complex assembly drives priming and fusion of secretory vesicles," EMBO J, vol. 25, no. 5, pp. 955-66, 2006. 3

[29] D. Fasshauer, W. K. Eliason, A. T. Brunger, and R. Jahn, "Identification of a minimal core of the synaptic snare complex sufficient for reversible assembly and disassembly," Biochemistry, vol. 37, no. 29, pp. 10354-62, 1998. 3

[30] G. Lonart and T. C. Sudhof, "Assembly of snare core complexes prior to neurotransmitter release sets the readily releasable pool of synaptic vesicles," J Biol Chem, vol. 275, no. 36, pp. 27703-7, 2000. 3 
[31] I. Dulubova, M. Khvotchev, S. Liu, I. Huryeva, T. C. Sudhof, and J. Rizo, "Munc18-1 binds directly to the neuronal snare complex," Proc Natl Acad Sci U S A, vol. 104, no. 8, pp. 2697-702, 2007. 3

[32] J. Shen, D. C. Tareste, F. Paumet, J. E. Rothman, and T. J. Melia, "Selective activation of cognate snarepins by sec1/munc18 proteins," Cell, vol. 128, no. 1, pp. 183-95, 2007. 3

[33] T. Sollner, S. W. Whiteheart, M. Brunner, H. Erdjument-Bromage, S. Geromanos, P. Tempst, and J. E. Rothman, "Snap receptors implicated in vesicle targeting and fusion," Nature, vol. 362, no. 6418, pp. 318-24, 1993. 3

[34] R. B. Sutton, D. Fasshauer, R. Jahn, and A. T. Brunger, "Crystal structure of a snare complex involved in synaptic exocytosis at 2.4 a resolution," Nature, vol. 395, no. 6700, pp. $347-53,1998$. 3

[35] X. Chen, D. R. Tomchick, E. Kovrigin, D. Arac, M. Machius, T. C. Sudhof, and J. Rizo, "Three-dimensional structure of the complexin/snare complex," Neuron, vol. 33, no. 3, pp. 397-409, 2002. 3

[36] K. Reim, M. Mansour, F. Varoqueaux, H. T. McMahon, T. C. Sudhof, N. Brose, and C. Rosenmund, "Complexins regulate a late step in ca2+-dependent neurotransmitter release," Cell, vol. 104, no. 1, pp. 71-81, 2001. 3

[37] C. G. Giraudo, W. S. Eng, T. J. Melia, and J. E. Rothman, "A clamping mechanism involved in snare-dependent exocytosis," Science, vol. 313, no. 5787, pp. 676-80, 2006. 3.,4

[38] M. Xue, Y. Q. Lin, H. Pan, K. Reim, H. Deng, H. J. Bellen, and C. Rosenmund, “Tilting the balance between facilitatory and inhibitory functions of mammalian and drosophila complexins orchestrates synaptic vesicle exocytosis," Neuron, vol. 64, no. 3, pp. 367-80, 2009. 3

[39] U. Ashery, F. Varoqueaux, T. Voets, A. Betz, P. Thakur, H. Koch, E. Neher, N. Brose, and J. Rettig, "Munc13-1 acts as a priming factor for large dense-core vesicles in bovine chromaffin cells," EMBO J, vol. 19, no. 14, pp. 3586-96, 2000. 3

[40] I. Augustin, C. Rosenmund, T. C. Sudhof, and N. Brose, "Munc13-1 is essential for fusion competence of glutamatergic synaptic vesicles," Nature, vol. 400, no. 6743, pp. 457-61, 1999. 3, 5 
[41] K. Broadie, H. J. Bellen, A. DiAntonio, J. T. Littleton, and T. L. Schwarz, "Absence of synaptotagmin disrupts excitation-secretion coupling during synaptic transmission," Proc Natl Acad Sci U S A, vol. 91, no. 22, pp. 10727-31, 1994. 3

[42] M. Geppert, Y. Goda, R. E. Hammer, C. Li, T. W. Rosahl, C. F. Stevens, and T. C. Sudhof, "Synaptotagmin i: a major ca2+ sensor for transmitter release at a central synapse," Cell, vol. 79, no. 4, pp. 717-27, 1994. 3

[43] J. T. Littleton, M. Stern, K. Schulze, M. Perin, and H. J. Bellen, "Mutational analysis of drosophila synaptotagmin demonstrates its essential role in ca(2+)-activated neurotransmitter release," Cell, vol. 74, no. 6, pp. 1125-34, 1993. 3

[44] B. A. Davletov and T. C. Sudhof, "A single c2 domain from synaptotagmin i is sufficient for high affinity ca2+/phospholipid binding," J Biol Chem, vol. 268, no. 35, pp. 2638690, 1993. 4

[45] I. Fernandez, D. Arac, J. Ubach, S. H. Gerber, O. Shin, Y. Gao, R. G. Anderson, T. C. Sudhof, and J. Rizo, "Three-dimensional structure of the synaptotagmin 1 c2b-domain: synaptotagmin 1 as a phospholipid binding machine," Neuron, vol. 32, no. 6, pp. 105769,2001 . 4

[46] E. R. Chapman, P. I. Hanson, S. An, and R. Jahn, "Ca2+ regulates the interaction between synaptotagmin and syntaxin 1," J Biol Chem, vol. 270, no. 40, pp. 23667-71, 1995. 4, 17

[47] G. Schiavo, G. Stenbeck, J. E. Rothman, and T. H. Sollner, "Binding of the synaptic vesicle v-snare, synaptotagmin, to the plasma membrane t-snare, snap-25, can explain docked vesicles at neurotoxin-treated synapses," Proc Natl Acad Sci U S A, vol. 94, no. 3, pp. 997-1001, 1997. 4, 82, 83

[48] H. Dai, N. Shen, D. Arac, and J. Rizo, "A quaternary snare-synaptotagmin-ca2+phospholipid complex in neurotransmitter release," J Mol Biol, vol. 367, no. 3, pp. 84863, 2007. 4

[49] X. Yang, Y. J. Kaeser-Woo, Z. P. Pang, W. Xu, and T. C. Sudhof, "Complexin clamps asynchronous release by blocking a secondary ca(2+) sensor via its accessory alpha helix," Neuron, vol. 68, no. 5, pp. 907-20, 2010. 4

[50] R. Jahn and R. H. Scheller, "Snares-engines for membrane fusion," Nat Rev Mol Cell Biol, vol. 7, no. 9, pp. 631-43, 2006. 4 
[51] T. Sollner, M. K. Bennett, S. W. Whiteheart, R. H. Scheller, and J. E. Rothman, "A protein assembly-disassembly pathway in vitro that may correspond to sequential steps of synaptic vesicle docking, activation, and fusion," Cell, vol. 75, no. 3, pp. 409-18, 1993. 4

[52] E. G. Gray, "Electron microscopy of presynaptic organelles of the spinal cord," J Anat, vol. 97, pp. 101-6, 1963. 4

[53] D. M. Landis, A. K. Hall, L. A. Weinstein, and T. S. Reese, "The organization of cytoplasm at the presynaptic active zone of a central nervous system synapse," Neuron, vol. 1, no. 3, pp. 201-9, 1988. 4

[54] M. L. Harlow, D. Ress, A. Stoschek, R. M. Marshall, and U. J. McMahan, "The architecture of active zone material at the frog's neuromuscular junction," Nature, vol. 409, no. 6819 , pp. 479-84, 2001. 4

[55] L. Siksou, P. Rostaing, J. P. Lechaire, T. Boudier, T. Ohtsuka, A. Fejtova, H. T. Kao, P. Greengard, E. D. Gundelfinger, A. Triller, and S. Marty, “Three-dimensional architecture of presynaptic terminal cytomatrix," J Neurosci, vol. 27, no. 26, pp. 6868-77, 2007. 4, 54

[56] R. Fernandez-Busnadiego, B. Zuber, U. E. Maurer, M. Cyrklaff, W. Baumeister, and V. Lucic, "Quantitative analysis of the native presynaptic cytomatrix by cryoelectron tomography,” J Cell Biol, vol. 188, no. 1, pp. 145-56, 2010. 4

[57] S. Brenner, "The genetics of caenorhabditis elegans," Genetics, vol. 77, no. 1, pp. 71-94, 1974. 5

[58] N. Brose, K. Hofmann, Y. Hata, and T. C. Sudhof, "Mammalian homologues of caenorhabditis elegans unc-13 gene define novel family of c2-domain proteins," J Biol Chem, vol. 270, no. 42, pp. 25273-80, 1995. 5

[59] H. Koch, K. Hofmann, and N. Brose, "Definition of munc13-homology-domains and characterization of a novel ubiquitously expressed munc13 isoform," Biochem J, vol. 349, no. Pt 1, pp. 247-53, 2000. 5

[60] C. Rosenmund, A. Sigler, I. Augustin, K. Reim, N. Brose, and J. S. Rhee, "Differential control of vesicle priming and short-term plasticity by munc13 isoforms," Neuron, vol. 33, no. 3, pp. 411-24, 2002. 5 
[61] F. Varoqueaux, A. Sigler, J. S. Rhee, N. Brose, C. Enk, K. Reim, and C. Rosenmund, "Total arrest of spontaneous and evoked synaptic transmission but normal synaptogenesis in the absence of munc13-mediated vesicle priming," Proc Natl Acad Sci U S A, vol. 99, no. 13, pp. 9037-42, 2002. 5, 9

[62] J. E. Richmond, W. S. Davis, and E. M. Jorgensen, "Unc-13 is required for synaptic vesicle fusion in c. elegans," Nat Neurosci, vol. 2, no. 11, pp. 959-64, 1999. 5

[63] B. Aravamudan, T. Fergestad, W. S. Davis, C. K. Rodesch, and K. Broadie, "Drosophila unc-13 is essential for synaptic transmission," Nat Neurosci, vol. 2, no. 11, pp. 965-71, 1999. 5

[64] S. Orita, A. Naito, G. Sakaguchi, M. Maeda, H. Igarashi, T. Sasaki, and Y. Takai, "Physical and functional interactions of doc2 and munc13 in ca2+-dependent exocytotic machinery," J Biol Chem, vol. 272, no. 26, pp. 16081-4, 1997. 5

[65] H. J. Junge, J. S. Rhee, O. Jahn, F. Varoqueaux, J. Spiess, M. N. Waxham, C. Rosenmund, and N. Brose, "Calmodulin and munc13 form a ca2+ sensor/effector complex that controls short-term synaptic plasticity," Cell, vol. 118, no. 3, pp. 389-401, 2004. 5

[66] G. Sakaguchi, S. Orita, A. Naito, M. Maeda, H. Igarashi, T. Sasaki, and Y. Takai, "A novel brain-specific isoform of beta spectrin: isolation and its interaction with munc13," Biochem Biophys Res Commun, vol. 248, no. 3, pp. 846-51, 1998. 5

[67] A. Betz, P. Thakur, H. J. Junge, U. Ashery, J. S. Rhee, V. Scheuss, C. Rosenmund, J. Rettig, and N. Brose, "Functional interaction of the active zone proteins munc13-1 and rim1 in synaptic vesicle priming," Neuron, vol. 30, no. 1, pp. 183-96, 2001. 5, 8, 83

[68] I. Dulubova, X. Lou, J. Lu, I. Huryeva, A. Alam, R. Schneggenburger, T. C. Sudhof, and J. Rizo, "A munc13/rim/rab3 tripartite complex: from priming to plasticity?," EMBO J, vol. 24, no. 16, pp. 2839-50, 2005. 5, 59, 82

[69] A. Betz, M. Okamoto, F. Benseler, and N. Brose, "Direct interaction of the rat unc-13 homologue munc13-1 with the $\mathrm{n}$ terminus of syntaxin," J Biol Chem, vol. 272, no. 4, pp. 2520-6, 1997. 5

[70] D. R. Stevens, Z. X. Wu, U. Matti, H. J. Junge, C. Schirra, U. Becherer, S. M. Wojcik, N. Brose, and J. Rettig, "Identification of the minimal protein domain required for priming activity of munc13-1," Curr Biol, vol. 15, no. 24, pp. 2243-8, 2005. 5 
[71] J. Basu, N. Shen, I. Dulubova, J. Lu, R. Guan, O. Guryev, N. V. Grishin, C. Rosenmund, and J. Rizo, "A minimal domain responsible for munc13 activity," Nat Struct Mol Biol, vol. 12, no. 11, pp. 1017-8, 2005. 5

[72] C. Ma, W. Li, Y. Xu, and J. Rizo, "Munc13 mediates the transition from the closed syntaxin-munc18 complex to the snare complex," Nat Struct Mol Biol, vol. 18, no. 5, pp. 542-9, 2011. 5

[73] J. Lu, M. Machius, I. Dulubova, H. Dai, T. C. Sudhof, D. R. Tomchick, and J. Rizo, "Structural basis for a munc13-1 homodimer to munc13-1/rim heterodimer switch," PLoS Biol, vol. 4, no. 7, p. e192, 2006. 5]

[74] O. H. Shin, J. Lu, J. S. Rhee, D. R. Tomchick, Z. P. Pang, S. M. Wojcik, M. CamachoPerez, N. Brose, M. Machius, J. Rizo, C. Rosenmund, and T. C. Sudhof, "Munc13 c2b domain is an activity-dependent ca2+ regulator of synaptic exocytosis," Nat Struct Mol Biol, vol. 17, no. 3, pp. 280-8, 2010. 5

[75] Y. Wang, S. Sugita, and T. C. Sudhof, "The rim/nim family of neuronal c2 domain proteins. interactions with rab3 and a new class of src homology 3 domain proteins," $J$ Biol Chem, vol. 275, no. 26, pp. 20033-44, 2000. 5, 82

[76] Y. Wang and T. C. Sudhof, "Genomic definition of rim proteins: evolutionary amplification of a family of synaptic regulatory proteins( small star, filled )," Genomics, vol. 81, no. 2, pp. 126-37, 2003. 5

[77] T. Coppola, S. Magnin-Luthi, V. Perret-Menoud, S. Gattesco, G. Schiavo, and R. Regazzi, "Direct interaction of the rab3 effector rim with ca2+ channels, snap-25, and synaptotagmin," J Biol Chem, vol. 276, no. 35, pp. 32756-62, 2001. 5

[78] Y. Wang, M. Okamoto, F. Schmitz, K. Hofmann, and T. C. Sudhof, "Rim is a putative rab3 effector in regulating synaptic-vesicle fusion," Nature, vol. 388, no. 6642, pp. 5938, 1997. 5, 8, 73

[79] E. O. Gracheva, G. Hadwiger, M. L. Nonet, and J. E. Richmond, "Direct interactions between c. elegans rab-3 and rim provide a mechanism to target vesicles to the presynaptic density," Neurosci Lett, vol. 444, no. 2, pp. 137-42, 2008. 5, 6, 59, 82

[80] Y. Han, P. S. Kaeser, T. C. Sudhof, and R. Schneggenburger, "Rim determines ca(2)+ channel density and vesicle docking at the presynaptic active zone," Neuron, vol. 69, no. 2, pp. 304-16, 2011. 5 
[81] C. Stigloher, H. Zhan, M. Zhen, J. Richmond, and J. L. Bessereau, "The presynaptic dense projection of the caenorhabditis elegans cholinergic neuromuscular junction localizes synaptic vesicles at the active zone through syd-2/liprin and unc-10/rim-dependent interactions," J Neurosci, vol. 31, no. 12, pp. 4388-96, 2011. 5, 7

[82] N. Calakos, S. Schoch, T. C. Sudhof, and R. C. Malenka, "Multiple roles for the active zone protein rim1alpha in late stages of neurotransmitter release," Neuron, vol. 42, no. 6 , pp. 889-96, 2004. 5

[83] R. M. Weimer, E. O. Gracheva, O. Meyrignac, K. G. Miller, J. E. Richmond, and J. L. Bessereau, "Unc-13 and unc-10/rim localize synaptic vesicles to specific membrane domains," J Neurosci, vol. 26, no. 31, pp. 8040-7, 2006. 5

[84] P. S. Kaeser, L. Deng, Y. Wang, I. Dulubova, X. Liu, J. Rizo, and T. C. Sudhof, "Rim proteins tether ca2+ channels to presynaptic active zones via a direct pdz-domain interaction," Cell, vol. 144, no. 2, pp. 282-95, 2011. 5, 6, 8, 83

[85] S. Schoch, P. E. Castillo, T. Jo, K. Mukherjee, M. Geppert, Y. Wang, F. Schmitz, R. C. Malenka, and T. C. Sudhof, "Rim1alpha forms a protein scaffold for regulating neurotransmitter release at the active zone," Nature, vol. 415, no. 6869, pp. 321-6, 2002. 5, 7 , 8,83

[86] T. Shibasaki, Y. Sunaga, K. Fujimoto, Y. Kashima, and S. Seino, "Interaction of atp sensor, camp sensor, ca2+ sensor, and voltage-dependent ca2+ channel in insulin granule exocytosis," J Biol Chem, vol. 279, no. 9, pp. 7956-61, 2004. 5, 7]

[87] T. Ohtsuka, E. Takao-Rikitsu, E. Inoue, M. Inoue, M. Takeuchi, K. Matsubara, M. Deguchi-Tawarada, K. Satoh, K. Morimoto, H. Nakanishi, and Y. Takai, "Cast: a novel protein of the cytomatrix at the active zone of synapses that forms a ternary complex with rim1 and munc13-1," J Cell Biol, vol. 158, no. 3, pp. 577-90, 2002. 5, 6, 8, 83

[88] Y. Wang, X. Liu, T. Biederer, and T. C. Sudhof, "A family of rim-binding proteins regulated by alternative splicing: Implications for the genesis of synaptic active zones," Proc Natl Acad Sci U S A, vol. 99, no. 22, pp. 14464-9, 2002. 5, 6, 73

[89] J. Lu, H. Li, Y. Wang, T. C. Sudhof, and J. Rizo, "Solution structure of the rim1alpha pdz domain in complex with an elks 1b c-terminal peptide," J Mol Biol, vol. 352, no. 2, pp. 455-66, 2005. 5, 6 
[90] L. Deng, P. S. Kaeser, W. Xu, and T. C. Sudhof, "Rim proteins activate vesicle priming by reversing autoinhibitory homodimerization of munc13," Neuron, vol. 69, no. 2, pp. 317-31, 2011. 6

[91] T. Nakata, Y. Kitamura, K. Shimizu, S. Tanaka, M. Fujimori, S. Yokoyama, K. Ito, and M. Emi, "Fusion of a novel gene, elks, to ret due to translocation $\mathrm{t}(10 ; 12)(\mathrm{q} 11 ; \mathrm{p} 13)$ in a papillary thyroid carcinoma," Genes Chromosomes Cancer, vol. 25, no. 2, pp. 97-103, 1999. 6

[92] E. Takao-Rikitsu, S. Mochida, E. Inoue, M. Deguchi-Tawarada, M. Inoue, T. Ohtsuka, and Y. Takai, "Physical and functional interaction of the active zone proteins, cast, rim1, and bassoon, in neurotransmitter release," J Cell Biol, vol. 164, no. 2, pp. 301-11, 2004. 6, 7, 8

[93] J. Ko, M. Na, S. Kim, J. R. Lee, and E. Kim, "Interaction of the erc family of rim-binding proteins with the liprin-alpha family of multidomain proteins," J Biol Chem, vol. 278, no. 43, pp. 42377-85, 2003. 6, 7, 8

[94] E. Inoue, M. Deguchi-Tawarada, E. Takao-Rikitsu, M. Inoue, I. Kitajima, T. Ohtsuka, and Y. Takai, "Elks, a protein structurally related to the active zone protein cast, is involved in ca2+-dependent exocytosis from pc12 cells," Genes Cells, vol. 11, no. 6, pp. 659-72, 2006. 6

[95] H. Nomura, T. Ohtsuka, S. Tadokoro, M. Tanaka, and N. Hirashima, "Involvement of elks, an active zone protein, in exocytotic release from rbl-2h3 cells," Cell Immunol, vol. 258 , no. 2, pp. 204-11, 2009. 6

[96] P. S. Kaeser, L. Deng, A. E. Chavez, X. Liu, P. E. Castillo, and T. C. Sudhof, "Elks2alpha/cast deletion selectively increases neurotransmitter release at inhibitory synapses," Neuron, vol. 64, no. 2, pp. 227-39, 2009. 6

[97] S. tom Dieck, L. Sanmarti-Vila, K. Langnaese, K. Richter, S. Kindler, A. Soyke, H. Wex, K. H. Smalla, U. Kampf, J. T. Franzer, M. Stumm, C. C. Garner, and E. D. Gundelfinger, "Bassoon, a novel zinc-finger cag/glutamine-repeat protein selectively localized at the active zone of presynaptic nerve terminals," J Cell Biol, vol. 142, no. 2, pp. 499-509, 1998. 7, 73

[98] S. D. Fenster, W. J. Chung, R. Zhai, C. Cases-Langhoff, B. Voss, A. M. Garner, U. Kaempf, S. Kindler, E. D. Gundelfinger, and C. C. Garner, "Piccolo, a presynaptic 
zinc finger protein structurally related to bassoon," Neuron, vol. 25, no. 1, pp. 203-14, 2000. 7

[99] X. Wang, M. Kibschull, M. M. Laue, B. Lichte, E. Petrasch-Parwez, and M. W. Kilimann, "Aczonin, a 550-kd putative scaffolding protein of presynaptic active zones, shares homology regions with rim and bassoon and binds profilin," J Cell Biol, vol. 147, no. 1, pp. 151-62, 1999. 7

[100] C. Serra-Pages, Q. G. Medley, M. Tang, A. Hart, and M. Streuli, "Liprins, a family of lar transmembrane protein-tyrosine phosphatase-interacting proteins," J Biol Chem, vol. 273, no. 25, pp. 15611-20, 1998. 7

[101] S. Kim, J. Ko, H. Shin, J. R. Lee, C. Lim, J. H. Han, W. D. Altrock, C. C. Garner, E. D. Gundelfinger, R. T. Premont, B. K. Kaang, and E. Kim, "The git family of proteins forms multimers and associates with the presynaptic cytomatrix protein piccolo," J Biol Chem, vol. 278, no. 8, pp. 6291-300, 2003. 7

[102] S. D. Fenster, M. M. Kessels, B. Qualmann, W. J. Chung, J. Nash, E. D. Gundelfinger, and C. C. Garner, "Interactions between piccolo and the actin/dynamin-binding protein abp1 link vesicle endocytosis to presynaptic active zones," J Biol Chem, vol. 278, no. 22, pp. 20268-77, 2003. 7

[103] W. D. Altrock, S. tom Dieck, M. Sokolov, A. C. Meyer, A. Sigler, C. Brakebusch, R. Fassler, K. Richter, T. M. Boeckers, H. Potschka, C. Brandt, W. Loscher, D. Grimberg, T. Dresbach, A. Hempelmann, H. Hassan, D. Balschun, J. U. Frey, J. H. Brandstatter, C. C. Garner, C. Rosenmund, and E. D. Gundelfinger, "Functional inactivation of a fraction of excitatory synapses in mice deficient for the active zone protein bassoon," Neuron, vol. 37, no. 5, pp. 787-800, 2003. 7

[104] R. Zhai, G. Olias, W. J. Chung, R. A. Lester, S. tom Dieck, K. Langnaese, M. R. Kreutz, S. Kindler, E. D. Gundelfinger, and C. C. Garner, "Temporal appearance of the presynaptic cytomatrix protein bassoon during synaptogenesis," Mol Cell Neurosci, vol. 15, no. 5, pp. 417-28, 2000. 7

[105] M. Shapira, R. G. Zhai, T. Dresbach, T. Bresler, V. I. Torres, E. D. Gundelfinger, N. E. Ziv, and C. C. Garner, "Unitary assembly of presynaptic active zones from piccolobassoon transport vesicles," Neuron, vol. 38, no. 2, pp. 237-52, 2003. 7

[106] S. tom Dieck, W. D. Altrock, M. M. Kessels, B. Qualmann, H. Regus, D. Brauner, A. Fejtova, O. Bracko, E. D. Gundelfinger, and J. H. Brandstatter, "Molecular dissection of 
the photoreceptor ribbon synapse: physical interaction of bassoon and ribeye is essential for the assembly of the ribbon complex," J Cell Biol, vol. 168, no. 5, pp. 825-36, 2005. 7

[107] O. Dick, S. tom Dieck, W. D. Altrock, J. Ammermuller, R. Weiler, C. C. Garner, E. D. Gundelfinger, and J. H. Brandstatter, "The presynaptic active zone protein bassoon is essential for photoreceptor ribbon synapse formation in the retina," Neuron, vol. 37, no. 5, pp. 775-86, 2003. 7

[108] S. Leal-Ortiz, C. L. Waites, R. Terry-Lorenzo, P. Zamorano, E. D. Gundelfinger, and C. C. Garner, "Piccolo modulation of synapsin1a dynamics regulates synaptic vesicle exocytosis," J Cell Biol, vol. 181, no. 5, pp. 831-46, 2008. 7

[109] K. Mukherjee, X. Yang, S. H. Gerber, H. B. Kwon, A. Ho, P. E. Castillo, X. Liu, and T. C. Sudhof, "Piccolo and bassoon maintain synaptic vesicle clustering without directly participating in vesicle exocytosis," Proc Natl Acad Sci U S A, vol. 107, no. 14, pp. 65049, 2010. 7

[110] E. Yeh, T. Kawano, R. M. Weimer, J. L. Bessereau, and M. Zhen, "Identification of genes involved in synaptogenesis using a fluorescent active zone marker in caenorhabditis elegans," J Neurosci, vol. 25, no. 15, pp. 3833-41, 2005. 7

[111] Y. Dai, H. Taru, S. L. Deken, B. Grill, B. Ackley, M. L. Nonet, and Y. Jin, "Syd-2 liprinalpha organizes presynaptic active zone formation through elks," Nat Neurosci, vol. 9, no. 12 , pp. $1479-87,2006.7$

[112] O. Olsen, K. A. Moore, M. Fukata, T. Kazuta, J. C. Trinidad, F. W. Kauer, M. Streuli, H. Misawa, A. L. Burlingame, R. A. Nicoll, and D. S. Bredt, "Neurotransmitter release regulated by a mals-liprin-alpha presynaptic complex," J Cell Biol, vol. 170, no. 7, pp. $1127-34,2005$. 7, 8,48

[113] M. Zhen and Y. Jin, "The liprin protein syd-2 regulates the differentiation of presynaptic termini in c. elegans," Nature, vol. 401, no. 6751, pp. 371-5, 1999. 7

[114] N. Kaufmann, J. DeProto, R. Ranjan, H. Wan, and D. Van Vactor, "Drosophila liprinalpha and the receptor phosphatase dlar control synapse morphogenesis," Neuron, vol. 34 , no. 1 , pp. $27-38,2002$. 7

[115] S. M. Kaech, C. W. Whitfield, and S. K. Kim, "The lin-2/lin-7/lin-10 complex mediates basolateral membrane localization of the c. elegans egf receptor let-23 in vulval epithelial cells," Cell, vol. 94, no. 6, pp. 761-71, 1998. 7,8 
[116] S. Butz, M. Okamoto, and T. C. Sudhof, "A tripartite protein complex with the potential to couple synaptic vesicle exocytosis to cell adhesion in brain," Cell, vol. 94, no. 6, pp. 773-82, 1998. 7

[117] Y. Hata, S. Butz, and T. C. Sudhof, "Cask: a novel dlg/psd95 homolog with an n-terminal calmodulin-dependent protein kinase domain identified by interaction with neurexins," J Neurosci, vol. 16, no. 8, pp. 2488-94, 1996. 7, 8, 73

[118] K. Jo, R. Derin, M. Li, and D. S. Bredt, "Characterization of mals/velis-1, -2, and -3: a family of mammalian lin-7 homologs enriched at brain synapses in association with the postsynaptic density-95/nmda receptor postsynaptic complex," J Neurosci, vol. 19, no. 11, pp. 4189-99, 1999. 7

[119] M. Setou, T. Nakagawa, D. H. Seog, and N. Hirokawa, "Kinesin superfamily motor protein kif17 and mlin-10 in nmda receptor-containing vesicle transport," Science, vol. 288, no. 5472 , pp. $1796-802,2000.7$

[120] M. Okamoto and T. C. Sudhof, "Mints, munc18-interacting proteins in synaptic vesicle exocytosis," J Biol Chem, vol. 272, no. 50, pp. 31459-64, 1997. 8,73

[121] M. B. Dalva, A. C. McClelland, and M. S. Kayser, "Cell adhesion molecules: signalling functions at the synapse," Nat Rev Neurosci, vol. 8, no. 3, pp. 206-20, 2007. 8

[122] M. Missler, W. Zhang, A. Rohlmann, G. Kattenstroth, R. E. Hammer, K. Gottmann, and T. C. Sudhof, "Alpha-neurexins couple ca2+ channels to synaptic vesicle exocytosis," Nature, vol. 423, no. 6943, pp. 939-48, 2003. 8

[123] K. Jungling, V. Eulenburg, R. Moore, R. Kemler, V. Lessmann, and K. Gottmann, "Ncadherin transsynaptically regulates short-term plasticity at glutamatergic synapses in embryonic stem cell-derived neurons," J Neurosci, vol. 26, no. 26, pp. 6968-78, 2006. 8

[124] C. Dillon and Y. Goda, "The actin cytoskeleton: integrating form and function at the synapse," Апnи Rev Neurosci, vol. 28, pp. 25-55, 2005. 9

[125] N. Hirokawa, K. Sobue, K. Kanda, A. Harada, and H. Yorifuji, "The cytoskeletal architecture of the presynaptic terminal and molecular structure of synapsin 1," J Cell Biol, vol. 108, no. 1, pp. 111-26, 1989. 9, 53, 83

[126] E. G. Gray, "Axo-somatic and axo-dendritic synapses of the cerebral cortex: an electron microscope study," J Anat, vol. 93, pp. 420-33, 1959. 9, 34 
[127] F. Varoqueaux, S. Jamain, and N. Brose, "Neuroligin 2 is exclusively localized to inhibitory synapses,” Eur J Cell Biol, vol. 83, no. 9, pp. 449-56, 2004. 9

[128] A. M. Craig, G. Banker, W. Chang, M. E. McGrath, and A. S. Serpinskaya, "Clustering of gephyrin at gabaergic but not glutamatergic synapses in cultured rat hippocampal neurons," J Neurosci, vol. 16, no. 10, pp. 3166-77, 1996. 9

[129] D. Gitler, Y. Takagishi, J. Feng, Y. Ren, R. M. Rodriguiz, W. C. Wetsel, P. Greengard, and G. J. Augustine, "Different presynaptic roles of synapsins at excitatory and inhibitory synapses," J Neurosci, vol. 24, no. 50, pp. 11368-80, 2004. 9

[130] M. Gronborg, N. J. Pavlos, I. Brunk, J. J. Chua, A. Munster-Wandowski, D. Riedel, G. Ahnert-Hilger, H. Urlaub, and R. Jahn, "Quantitative comparison of glutamatergic and gabaergic synaptic vesicles unveils selectivity for few proteins including mal2, a novel synaptic vesicle protein," J Neurosci, vol. 30, no. 1, pp. 2-12, 2010. 9, 42

[131] H. Husi, M. A. Ward, J. S. Choudhary, W. P. Blackstock, and S. G. Grant, "Proteomic analysis of nmda receptor-adhesion protein signaling complexes," Nat Neurosci, vol. 3, no. 7, pp. 661-9, 2000. 10

[132] R. S. Walikonis, O. N. Jensen, M. Mann, J. Provance, D. W., J. A. Mercer, and M. B. Kennedy, "Identification of proteins in the postsynaptic density fraction by mass spectrometry," J Neurosci, vol. 20, no. 11, pp. 4069-80, 2000. 10

[133] K. W. Li, M. P. Hornshaw, R. C. Van Der Schors, R. Watson, S. Tate, B. Casetta, C. R. Jimenez, Y. Gouwenberg, E. D. Gundelfinger, K. H. Smalla, and A. B. Smit, "Proteomics analysis of rat brain postsynaptic density. implications of the diverse protein functional groups for the integration of synaptic physiology," J Biol Chem, vol. 279, no. 2, pp. 9871002, 2004. 10, 73, 75

[134] K. Li, M. P. Hornshaw, J. van Minnen, K. H. Smalla, E. D. Gundelfinger, and A. B. Smit, "Organelle proteomics of rat synaptic proteins: correlation-profiling by isotope-coded affinity tagging in conjunction with liquid chromatography-tandem mass spectrometry to reveal post-synaptic density specific proteins," J Proteome Res, vol. 4, no. 3, pp. 72533, 2005. 10, 12

[135] J. Peng, M. J. Kim, D. Cheng, D. M. Duong, S. P. Gygi, and M. Sheng, "Semiquantitative proteomic analysis of rat forebrain postsynaptic density fractions by mass spectrometry," J Biol Chem, vol. 279, no. 20, pp. 21003-11, 2004. 10, 12, 73, 75 
[136] H. D. Coughenour, R. S. Spaulding, and C. M. Thompson, "The synaptic vesicle proteome: a comparative study in membrane protein identification," Proteomics, vol. 4, no. 10 , pp. $3141-55,2004$. 10

[137] J. Burre, T. Beckhaus, H. Schagger, C. Corvey, S. Hofmann, M. Karas, H. Zimmermann, and W. Volknandt, "Analysis of the synaptic vesicle proteome using three gel-based protein separation techniques," Proteomics, vol. 6, no. 23, pp. 6250-62, 2006. 10, 45

[138] S. Takamori, M. Holt, K. Stenius, E. A. Lemke, M. Gronborg, D. Riedel, H. Urlaub, S. Schenck, B. Brugger, P. Ringler, S. A. Muller, B. Rammner, F. Grater, J. S. Hub, B. L. De Groot, G. Mieskes, Y. Moriyama, J. Klingauf, H. Grubmuller, J. Heuser, F. Wieland, and R. Jahn, "Molecular anatomy of a trafficking organelle," Cell, vol. 127, no. 4, pp. 831-46, 2006. 10, 11, 45, 46, 78

[139] J. S. Andersen, C. J. Wilkinson, T. Mayor, P. Mortensen, E. A. Nigg, and M. Mann, "Proteomic characterization of the human centrosome by protein correlation profiling," Nature, vol. 426, no. 6966, pp. 570-4, 2003. 10, 11,78

[140] S. P. Gygi, B. Rist, S. A. Gerber, F. Turecek, M. H. Gelb, and R. Aebersold, "Quantitative analysis of complex protein mixtures using isotope-coded affinity tags," Nat Biotechnol, vol. 17 , no. 10, pp. 994-9, 1999.

[141] P. L. Ross, Y. N. Huang, J. N. Marchese, B. Williamson, K. Parker, S. Hattan, N. Khainovski, S. Pillai, S. Dey, S. Daniels, S. Purkayastha, P. Juhasz, S. Martin, M. BartletJones, F. He, A. Jacobson, and D. J. Pappin, "Multiplexed protein quantitation in saccharomyces cerevisiae using amine-reactive isobaric tagging reagents," Mol Cell Proteomics, vol. 3, no. 12, pp. 1154-69, 2004. 12, 44

[142] S. A. Gerber, J. Rush, O. Stemman, M. W. Kirschner, and S. P. Gygi, "Absolute quantification of proteins and phosphoproteins from cell lysates by tandem ms," Proc Natl Acad Sci U S A, vol. 100, no. 12, pp. 6940-5, 2003. 12

[143] S. E. Ong, B. Blagoev, I. Kratchmarova, D. B. Kristensen, H. Steen, A. Pandey, and M. Mann, "Stable isotope labeling by amino acids in cell culture, silac, as a simple and accurate approach to expression proteomics," Mol Cell Proteomics, vol. 1, no. 5, pp. 376-86, 2002. 12

[144] R. P. Munton, R. Tweedie-Cullen, M. Livingstone-Zatchej, F. Weinandy, M. Waidelich, D. Longo, P. Gehrig, F. Potthast, D. Rutishauser, B. Gerrits, C. Panse, R. Schlapbach, and I. M. Mansuy, "Qualitative and quantitative analyses of protein phosphorylation in 
naive and stimulated mouse synaptosomal preparations," Mol Cell Proteomics, vol. 6, no. 2, pp. 283-93, 2007. 13

[145] J. C. Trinidad, C. G. Specht, A. Thalhammer, R. Schoepfer, and A. L. Burlingame, "Comprehensive identification of phosphorylation sites in postsynaptic density preparations," Mol Cell Proteomics, vol. 5, no. 5, pp. 914-22, 2006. 13

[146] V. P. Whittaker, I. A. Michaelson, and R. J. Kirkland, "The separation of synaptic vesicles from nerve-ending particles ('synaptosomes')," Biochem J, vol. 90, no. 2, pp. 293303, 1964. 13, 36

[147] J. W. Hell, P. R. Maycox, H. Stadler, and R. Jahn, "Uptake of gaba by rat brain synaptic vesicles isolated by a new procedure," EMBO J, vol. 7, no. 10, pp. 3023-9, 1988. 13

[148] M. L. Dustin and D. R. Colman, "Neural and immunological synaptic relations," Science, vol. 298, no. 5594, pp. 785-9, 2002. 13

[149] G. Fischer von Mollard, T. C. Sudhof, and R. Jahn, "A small gtp-binding protein dissociates from synaptic vesicles during exocytosis," Nature, vol. 349, no. 6304, pp. 79-81, 1991. 14, 23, 29, 30, 31, 59

[150] R. K. Carlin, D. J. Grab, R. S. Cohen, and P. Siekevitz, "Isolation and characterization of postsynaptic densities from various brain regions: enrichment of different types of postsynaptic densities," J Cell Biol, vol. 86, no. 3, pp. 831-45, 1980. 14, 34

[151] G. R. Phillips, J. K. Huang, Y. Wang, H. Tanaka, L. Shapiro, W. Zhang, W. S. Shan, K. Arndt, M. Frank, R. E. Gordon, M. A. Gawinowicz, Y. Zhao, and D. R. Colman, "The presynaptic particle web: ultrastructure, composition, dissolution, and reconstitution," Neuron, vol. 32, no. 1, pp. 63-77, 2001. 14, 74, 77, 78

[152] G. R. Phillips, L. Florens, H. Tanaka, Z. Z. Khaing, L. Fidler, r. Yates, J. R., and D. R. Colman, "Proteomic comparison of two fractions derived from the transsynaptic scaffold," J Neurosci Res, vol. 81, no. 6, pp. 762-75, 2005. 14, 74, 75, 77, 81

[153] M. Morciano, T. Beckhaus, M. Karas, H. Zimmermann, and W. Volknandt, "The proteome of the presynaptic active zone: from docked synaptic vesicles to adhesion molecules and maxi-channels," J Neurochem, vol. 108, no. 3, pp. 662-75, 2009. 14, 74, 76, 77, 78, 79 
[154] R. Jahn, W. Schiebler, C. Ouimet, and P. Greengard, "A 38,000-dalton membrane protein (p38) present in synaptic vesicles," Proc Natl Acad Sci U S A, vol. 82, no. 12, pp. 413741, 1985. 17

[155] L. Edelmann, P. I. Hanson, E. R. Chapman, and R. Jahn, "Synaptobrevin binding to synaptophysin: a potential mechanism for controlling the exocytotic fusion machine," EMBO J, vol. 14, no. 2, pp. 224-31, 1995. 17

[156] N. Brose, A. G. Petrenko, T. C. Sudhof, and R. Jahn, "Synaptotagmin: a calcium sensor on the synaptic vesicle surface," Science, vol. 256, no. 5059, pp. 1021-5, 1992. 17

[157] N. Brose, G. W. Huntley, Y. Stern-Bach, G. Sharma, J. H. Morrison, and S. F. Heinemann, "Differential assembly of coexpressed glutamate receptor subunits in neurons of rat cerebral cortex," J Biol Chem, vol. 269, no. 24, pp. 16780-4, 1994. 17

[158] S. Takamori, J. S. Rhee, C. Rosenmund, and R. Jahn, "Identification of differentiationassociated brain-specific phosphate transporter as a second vesicular glutamate transporter (vglut2)," J Neurosci, vol. 21, no. 22, p. RC182, 2001. 17

[159] S. a. Russel, "Molecular cloning: A laboratory manual," Cold Spring Harbor Laboratory Press, vol. 1-3, 2001. 19

[160] F. Peter, C. Nuoffer, I. Schalk, and W. E. Balch, "Expression and purification of recombinant his6-tagged guanine nucleotide dissociation inhibitor and formation of its rab1 complex," Methods Enzymol, vol. 257, pp. 80-3, 1995. 19

[161] P. K. Smith, R. I. Krohn, G. T. Hermanson, A. K. Mallia, F. H. Gartner, M. D. Provenzano, E. K. Fujimoto, N. M. Goeke, B. J. Olson, and D. C. Klenk, "Measurement of protein using bicinchoninic acid," Anal Biochem, vol. 150, no. 1, pp. 76-85, 1985. 20

[162] P. J. Meberg and M. W. Miller, "Culturing hippocampal and cortical neurons," Methods Cell Biol, vol. 71, pp. 111-27, 2003. 20

[163] H. Schagger and G. von Jagow, "Tricine-sodium dodecyl sulfate-polyacrylamide gel electrophoresis for the separation of proteins in the range from 1 to $100 \mathrm{kda}$," Anal Biochem, vol. 166, no. 2, pp. 368-79, 1987. 22

[164] H. Schagger, “Tricine-sds-page," Nat Protoc, vol. 1, no. 1, pp. 16-22, 2006. 22

[165] H. Towbin, T. Staehelin, and J. Gordon, "Immunoblotting in the clinical laboratory," $J$ Clin Chem Clin Biochem, vol. 27, no. 8, pp. 495-501, 1989. 22 
[166] C. Bordier, "Phase separation of integral membrane proteins in triton x-114 solution," $J$ Biol Chem, vol. 256, no. 4, pp. 1604-7, 1981. 22, 66

[167] P. M. Burger, E. Mehl, P. L. Cameron, P. R. Maycox, M. Baumert, F. Lottspeich, P. De Camilli, and R. Jahn, "Synaptic vesicles immunoisolated from rat cerebral cortex contain high levels of glutamate," Neuron, vol. 3, no. 6, pp. 715-20, 1989. 23

[168] K. W. Li, S. Miller, O. Klychnikov, M. Loos, J. Stahl-Zeng, S. Spijker, M. Mayford, and A. B. Smit, "Quantitative proteomics and protein network analysis of hippocampal synapses of camkiialpha mutant mice," J Proteome Res, vol. 6, no. 8, pp. 3127-33, 2007. 25

[169] T. E. Thingholm and M. R. Larsen, "The use of titanium dioxide micro-columns to selectively isolate phosphopeptides from proteolytic digests," Methods Mol Biol, vol. 527, pp. 57-66, xi, 2009. 26

[170] J. H. Chou and R. Jahn, "Binding of rab3a to synaptic vesicles," J Biol Chem, vol. 275, no. 13 , pp. 9433-40, 2000. 27, 59

[171] E. G. Gray and V. P. Whittaker, "The isolation of nerve endings from brain: an electronmicroscopic study of cell fragments derived by homogenization and centrifugation," $J$ Anat, vol. 96, pp. 79-88, 1962. 29

[172] C. W. Cotman, G. Banker, L. Churchill, and D. Taylor, "Isolation of postsynaptic densities from rat brain," J Cell Biol, vol. 63, no. 2 Pt 1, pp. 441-55, 1974. 34,73

[173] M. Morciano, J. Burre, C. Corvey, M. Karas, H. Zimmermann, and W. Volknandt, "Immunoisolation of two synaptic vesicle pools from synaptosomes: a proteomics analysis," J Neurochem, vol. 95, no. 6, pp. 1732-45, 2005. 36, 40, 46, 74, 77

[174] G. A. Davis and F. E. Bloom, "Subcellular particles separated through a histochemical reaction," Anal Biochem, vol. 51, no. 2, pp. 429-35, 1973. 39

[175] D. H. Jones and A. I. Matus, "Isolation of synaptic plasma membrane from brain by combined flotation-sedimentation density gradient centrifugation," Biochim Biophys Acta, vol. 356, no. 3, pp. 276-87, 1974. 39

[176] C. W. Cotman and D. A. Matthews, "Synaptic plasma membranes from rat brain synaptosomes: isolation and partial characterization," Biochim Biophys Acta, vol. 249, no. 2, pp. 380-94, 1971. 39 
[177] D. J. Pagliarini, S. E. Calvo, B. Chang, S. A. Sheth, S. B. Vafai, S. E. Ong, G. A. Walford, C. Sugiana, A. Boneh, W. K. Chen, D. E. Hill, M. Vidal, J. G. Evans, D. R. Thorburn, S. A. Carr, and V. K. Mootha, "A mitochondrial protein compendium elucidates complex i disease biology," Cell, vol. 134, no. 1, pp. 112-23, 2008. 44

[178] T. Kremer, C. Kempf, N. Wittenmayer, R. Nawrotzki, T. Kuner, J. Kirsch, and T. Dresbach, "Mover is a novel vertebrate-specific presynaptic protein with differential distribution at subsets of cns synapses," FEBS Lett, vol. 581, no. 24, pp. 4727-33, 2007. 46

[179] M. Zerial and H. McBride, "Rab proteins as membrane organizers," Nat Rev Mol Cell Biol, vol. 2, no. 2, pp. 107-17, 2001. 46

[180] C. Geumann, M. Gronborg, M. Hellwig, H. Martens, and R. Jahn, "A sandwich enzymelinked immunosorbent assay for the quantification of insoluble membrane and scaffold proteins," Anal Biochem, vol. 402, no. 2, pp. 161-9, 2010. 46

[181] A. Simonsen, B. Bremnes, E. Ronning, R. Aasland, and H. Stenmark, "Syntaxin-16, a putative golgi t-snare," Eur J Cell Biol, vol. 75, no. 3, pp. 223-31, 1998. 49

[182] R. Robitaille, M. L. Garcia, G. J. Kaczorowski, and M. P. Charlton, "Functional colocalization of calcium and calcium-gated potassium channels in control of transmitter release," Neuron, vol. 11, no. 4, pp. 645-55, 1993. 50

[183] H. G. Knaus, C. Schwarzer, R. O. Koch, A. Eberhart, G. J. Kaczorowski, H. Glossmann, F. Wunder, O. Pongs, M. L. Garcia, and G. Sperk, "Distribution of high-conductance ca(2+)-activated k+ channels in rat brain: targeting to axons and nerve terminals," $J$ Neurosci, vol. 16, no. 3, pp. 955-63, 1996. 50

[184] H. Hu, L. R. Shao, S. Chavoshy, N. Gu, M. Trieb, R. Behrens, P. Laake, O. Pongs, H. G. Knaus, O. P. Ottersen, and J. F. Storm, "Presynaptic ca2+-activated k+ channels in glutamatergic hippocampal terminals and their role in spike repolarization and regulation of transmitter release," J Neurosci, vol. 21, no. 24, pp. 9585-97, 2001. 50

[185] Z. Huang, R. Lujan, I. Kadurin, V. N. Uebele, J. J. Renger, A. C. Dolphin, and M. M. Shah, "Presynaptic hen1 channels regulate ca(v)3.2 activity and neurotransmission at select cortical synapses," Nat Neurosci, 2011. 50

[186] M. Juhaszova, P. Church, M. P. Blaustein, and E. F. Stanley, "Location of calcium transporters at presynaptic terminals," Eur J Neurosci, vol. 12, no. 3, pp. 839-46, 2000. 51, 79 
[187] T. Jean, C. Frelin, P. Vigne, P. Barbry, and M. Lazdunski, "Biochemical properties of the nat/h+ exchange system in rat brain synaptosomes. interdependence of internal and external ph control of the exchange activity," J Biol Chem, vol. 260, no. 17, pp. 9678-84, 1985. 51

[188] L. E. Trudeau, V. Parpura, and P. G. Haydon, "Activation of neurotransmitter release in hippocampal nerve terminals during recovery from intracellular acidification," J Neurophysiol, vol. 81, no. 6, pp. 2627-35, 1999. 51

[189] A. Minelli, N. C. Brecha, C. Karschin, S. DeBiasi, and F. Conti, "Gat-1, a high-affinity gaba plasma membrane transporter, is localized to neurons and astroglia in the cerebral cortex," J Neurosci, vol. 15, no. 11, pp. 7734-46, 1995. 51

[190] F. A. Chaudhry, K. P. Lehre, M. van Lookeren Campagne, O. P. Ottersen, N. C. Danbolt, and J. Storm-Mathisen, "Glutamate transporters in glial plasma membranes: highly differentiated localizations revealed by quantitative ultrastructural immunocytochemistry," Neuron, vol. 15, no. 3, pp. 711-20, 1995. 51

[191] E. M. Rose, J. C. Koo, J. E. Antflick, S. M. Ahmed, S. Angers, and D. R. Hampson, "Glutamate transporter coupling to na,k-atpase," J Neurosci, vol. 29, no. 25, pp. 814355, 2009. 51,79

[192] L. Aigner and P. Caroni, "Depletion of 43-kd growth-associated protein in primary sensory neurons leads to diminished formation and spreading of growth cones," J Cell Biol, vol. 123 , no. 2, pp. 417-29, 1993. 51

[193] J. H. Skene, R. D. Jacobson, G. J. Snipes, C. B. McGuire, J. J. Norden, and J. A. Freeman, "A protein induced during nerve growth (gap-43) is a major component of growthcone membranes," Science, vol. 233, no. 4765, pp. 783-6, 1986. 51

[194] C. Lagenaur, V. Kunemund, G. Fischer, S. Fushiki, and M. Schachner, "Monoclonal m6 antibody interferes with neurite extension of cultured neurons," J Neurobiol, vol. 23, no. 1, pp. 71-88, 1992. 51

[195] B. Cooper, H. B. Werner, and G. Flugge, "Glycoprotein m6a is present in glutamatergic axons in adult rat forebrain and cerebellum," Brain Res, vol. 1197, pp. 1-12, 2008. 51

[196] W. Stohl and N. K. Gonatas, "Distribution of the thy-1 antigen in cellular and subcellular fractions of adult mouse brain," J Immunol, vol. 119, no. 2, pp. 422-7, 1977. 51 
[197] P. Doherty, A. Singh, G. Rimon, S. R. Bolsover, and F. S. Walsh, "Thy-1 antibodytriggered neurite outgrowth requires an influx of calcium into neurons via n- and l-type calcium channels," J Cell Biol, vol. 122, no. 1, pp. 181-9, 1993. 51

[198] H. Umemori and J. R. Sanes, "Signal regulatory proteins (sirps) are secreted presynaptic organizing molecules," J Biol Chem, vol. 283, no. 49, pp. 34053-61, 2008. 51

[199] S. Yamada, S. Pokutta, F. Drees, W. I. Weis, and W. J. Nelson, "Deconstructing the cadherin-catenin-actin complex," Cell, vol. 123, no. 5, pp. 889-901, 2005. 52

[200] J. Bai, Z. Hu, J. S. Dittman, E. C. Pym, and J. M. Kaplan, "Endophilin functions as a membrane-bending molecule and is delivered to endocytic zones by exocytosis," Cell, vol. 143 , no. 3, pp. 430-41, 2010. 53

[201] O. O. Glebov, N. A. Bright, and B. J. Nichols, "Flotillin-1 defines a clathrin-independent endocytic pathway in mammalian cells," Nat Cell Biol, vol. 8, no. 1, pp. 46-54, 2006. 53

[202] T. Schulte, K. A. Paschke, U. Laessing, F. Lottspeich, and C. A. Stuermer, "Reggie-1 and reggie-2, two cell surface proteins expressed by retinal ganglion cells during axon regeneration," Development, vol. 124, no. 2, pp. 577-87, 1997. 53

[203] S. O. Deininger, L. Rajendran, F. Lottspeich, M. Przybylski, H. Illges, C. A. Stuermer, and A. Reuter, "Identification of teleost thy-1 and association with the microdomain/lipid raft reggie proteins in regenerating cns axons," Mol Cell Neurosci, vol. 22, no. 4, pp. 544-54, 2003. 53

[204] M. Watanabe, K. Nomura, A. Ohyama, R. Ishikawa, Y. Komiya, K. Hosaka, E. Yamauchi, H. Taniguchi, N. Sasakawa, K. Kumakura, T. Ushiki, O. Sato, M. Ikebe, and M. Igarashi, "Myosin-va regulates exocytosis through the submicromolar ca2+dependent binding of syntaxin-1a," Mol Biol Cell, vol. 16, no. 10, pp. 4519-30, 2005. 53

[205] T. Wollert, A. Patel, Y. L. Lee, J. Provance, D. W., V. E. Vought, M. S. Cosgrove, J. A. Mercer, and G. M. Langford, "Myosin5a tail associates directly with rab3a-containing compartments in neurons," J Biol Chem, vol. 286, no. 16, pp. 14352-61, 2011. 53, 82

[206] L. Zhu, R. Vranckx, P. Khau Van Kien, A. Lalande, N. Boisset, F. Mathieu, M. Wegman, L. Glancy, J. M. Gasc, F. Brunotte, P. Bruneval, J. E. Wolf, J. B. Michel, and X. Jeunemaitre, "Mutations in myosin heavy chain 11 cause a syndrome associating thoracic aor- 
tic aneurysm/aortic dissection and patent ductus arteriosus," Nat Genet, vol. 38, no. 3, pp. 343-9, 2006. 54

[207] C. Zhao, J. Takita, Y. Tanaka, M. Setou, T. Nakagawa, S. Takeda, H. W. Yang, S. Terada, T. Nakata, Y. Takei, M. Saito, S. Tsuji, Y. Hayashi, and N. Hirokawa, "Charcotmarie-tooth disease type 2a caused by mutation in a microtubule motor kif1bbeta," Cell, vol. 105 , no. 5, pp. 587-97, 2001. 54

[208] C. L. Beites, K. A. Campbell, and W. S. Trimble, "The septin sept5/cdcrel-1 competes with alpha-snap for binding to the snare complex," Biochem J, vol. 385, no. Pt 2, pp. 347-53, 2005. 54

[209] Y. M. Yang, M. J. Fedchyshyn, G. Grande, J. Aitoubah, C. W. Tsang, H. Xie, C. A. Ackerley, W. S. Trimble, and L. Y. Wang, "Septins regulate developmental switching from microdomain to nanodomain coupling of $\mathrm{ca}(2+)$ influx to neurotransmitter release at a central synapse," Neuron, vol. 67, no. 1, pp. 100-15, 2010. 54

[210] J. Xue, C. W. Tsang, W. P. Gai, C. S. Malladi, W. S. Trimble, J. A. Rostas, and P. J. Robinson, "Septin 3 (g-septin) is a developmentally regulated phosphoprotein enriched in presynaptic nerve terminals," J Neurochem, vol. 91, no. 3, pp. 579-90, 2004. 54

[211] W. J. Nelson and P. J. Veshnock, "Ankyrin binding to (na+ + k+)atpase and implications for the organization of membrane domains in polarized cells," Nature, vol. 328, no. 6130 , pp. 533-6, 1987. 54

[212] Z. P. Li, E. P. Burke, J. S. Frank, V. Bennett, and K. D. Philipson, "The cardiac natca2+ exchanger binds to the cytoskeletal protein ankyrin," J Biol Chem, vol. 268, no. 16, pp. 11489-91, 1993. 54

[213] K. Kizhatil, J. Q. Davis, L. Davis, J. Hoffman, B. L. Hogan, and V. Bennett, "Ankyrin-g is a molecular partner of e-cadherin in epithelial cells and early embryos," J Biol Chem, vol. 282, no. 36, pp. 26552-61, 2007. 54

[214] D. A. Brown and T. S. Sihra, "Presynaptic signaling by heterotrimeric g-proteins," Handb Exp Pharmacol, no. 184, pp. 207-60, 2008. 54

[215] L. Sun, M. A. Bittner, and R. W. Holz, "Rim, a component of the presynaptic active zone and modulator of exocytosis, binds 14-3-3 through its n terminus," J Biol Chem, vol. 278, no. 40, pp. 38301-9, 2003. 54 
[216] Y. Li, Y. Wu, and Y. Zhou, "Modulation of inactivation properties of cav2.2 channels by 14-3-3 proteins," Neuron, vol. 51, no. 6, pp. 755-71, 2006. 54

[217] S. I. Walaas, F. S. Gorelick, and P. Greengard, "Presence of calcium/calmodulindependent protein kinase ii in nerve terminals of rat brain," Synapse, vol. 3, no. 4, pp. 356-62, 1989. 54

[218] C. C. Ouimet, T. L. McGuinness, and P. Greengard, "Immunocytochemical localization of calcium/calmodulin-dependent protein kinase ii in rat brain," Proc Natl Acad Sci U S A, vol. 81 , no. 17 , pp. $5604-8,1984$. 54

[219] Q. Liu, B. Chen, Q. Ge, and Z. W. Wang, "Presynaptic ca2+/calmodulin-dependent protein kinase ii modulates neurotransmitter release by activating bk channels at caenorhabditis elegans neuromuscular junction," J Neurosci, vol. 27, no. 39, pp. 10404-13, 2007. 54

[220] O. Ullrich, H. Stenmark, K. Alexandrov, L. A. Huber, K. Kaibuchi, T. Sasaki, Y. Takai, and M. Zerial, "Rab gdp dissociation inhibitor as a general regulator for the membrane association of rab proteins," J Biol Chem, vol. 268, no. 24, pp. 18143-50, 1993. 59

[221] S. Araki, A. Kikuchi, Y. Hata, M. Isomura, and Y. Takai, "Regulation of reversible binding of smg p25a, a ras p21-like gtp-binding protein, to synaptic plasma membranes and vesicles by its specific regulatory protein, gdp dissociation inhibitor," J Biol Chem, vol. 265 , no. 22, pp. 13007-15, 1990. 59

[222] W. L. Coleman, C. A. Bill, and M. Bykhovskaia, "Rab3a deletion reduces vesicle docking and transmitter release at the mouse diaphragm synapse," Neuroscience, vol. 148, no. 1, pp. 1-6, 2007. 59

[223] F. Peter, C. Nuoffer, I. Schalk, and W. E. Balch, "Expression and purification of recombinant his6-tagged guanine nucleotide dissociation inhibitor and formation of its rab1 complex," Methods Enzymol, vol. 257, pp. 80-3, 1995. 59

[224] E. R. Graf, R. W. Daniels, R. W. Burgess, T. L. Schwarz, and A. DiAntonio, "Rab3 dynamically controls protein composition at active zones," Neuron, vol. 64, no. 5, pp. 66377, 2009. 63

[225] J. Schultz, F. Milpetz, P. Bork, and C. P. Ponting, "Smart, a simple modular architecture research tool: identification of signaling domains," Proc Natl Acad Sci U S A, vol. 95, no. 11 , pp. 5857-64, 1998. 65 
[226] I. Letunic, T. Doerks, and P. Bork, "Smart 6: recent updates and new developments," Nucleic Acids Res, vol. 37, no. Database issue, pp. D229-32, 2009. 65

[227] A. Krogh, B. Larsson, G. von Heijne, and E. L. Sonnhammer, "Predicting transmembrane protein topology with a hidden markov model: application to complete genomes," J Mol Biol, vol. 305, no. 3, pp. 567-80, 2001. 65

[228] G. E. Tusnady and I. Simon, "The hmmtop transmembrane topology prediction server," Bioinformatics, vol. 17, no. 9, pp. 849-50, 2001. 65

[229] M. Cserzo, E. Wallin, I. Simon, G. von Heijne, and A. Elofsson, "Prediction of transmembrane alpha-helices in prokaryotic membrane proteins: the dense alignment surface method," Protein Eng, vol. 10, no. 6, pp. 673-6, 1997. 65

[230] C. Notredame, D. G. Higgins, and J. Heringa, "T-coffee: A novel method for fast and accurate multiple sequence alignment," J Mol Biol, vol. 302, no. 1, pp. 205-17, 2000. 65

[231] E. S. Lein, M. J. Hawrylycz, N. Ao, M. Ayres, A. Bensinger, A. Bernard, A. F. Boe, M. S. Boguski, K. S. Brockway, E. J. Byrnes, L. Chen, T. M. Chen, M. C. Chin, J. Chong, B. E. Crook, A. Czaplinska, C. N. Dang, S. Datta, N. R. Dee, A. L. Desaki, T. Desta, E. Diep, T. A. Dolbeare, M. J. Donelan, H. W. Dong, J. G. Dougherty, B. J. Duncan, A. J. Ebbert, G. Eichele, L. K. Estin, C. Faber, B. A. Facer, R. Fields, S. R. Fischer, T. P. Fliss, C. Frensley, S. N. Gates, K. J. Glattfelder, K. R. Halverson, M. R. Hart, J. G. Hohmann, M. P. Howell, D. P. Jeung, R. A. Johnson, P. T. Karr, R. Kawal, J. M. Kidney, R. H. Knapik, C. L. Kuan, J. H. Lake, A. R. Laramee, K. D. Larsen, C. Lau, T. A. Lemon, A. J. Liang, Y. Liu, L. T. Luong, J. Michaels, J. J. Morgan, R. J. Morgan, M. T. Mortrud, N. F. Mosqueda, L. L. Ng, R. Ng, G. J. Orta, C. C. Overly, T. H. Pak, S. E. Parry, S. D. Pathak, O. C. Pearson, R. B. Puchalski, Z. L. Riley, H. R. Rockett, S. A. Rowland, J. J. Royall, M. J. Ruiz, N. R. Sarno, K. Schaffnit, N. V. Shapovalova, T. Sivisay, C. R. Slaughterbeck, S. C. Smith, K. A. Smith, B. I. Smith, A. J. Sodt, N. N. Stewart, K. R. Stumpf, S. M. Sunkin, M. Sutram, A. Tam, C. D. Teemer, C. Thaller, C. L. Thompson, L. R. Varnam, A. Visel, R. M. Whitlock, P. E. Wohnoutka, C. K. Wolkey, V. Y. Wong, M. Wood, et al., "Genome-wide atlas of gene expression in the adult mouse brain," Nature, vol. 445, no. 7124, pp. 168-76, 2007. 65

[232] Y. Fujiki, A. L. Hubbard, S. Fowler, and P. B. Lazarow, "Isolation of intracellular membranes by means of sodium carbonate treatment: application to endoplasmic reticulum," J Cell Biol, vol. 93, no. 1, pp. 97-102, 1982. 66 
[233] B. A. Jordan, B. D. Fernholz, M. Boussac, C. Xu, G. Grigorean, E. B. Ziff, and T. A. Neubert, "Identification and verification of novel rodent postsynaptic density proteins," Mol Cell Proteomics, vol. 3, no. 9, pp. 857-71, 2004. 73

[234] Y. Yoshimura, Y. Yamauchi, T. Shinkawa, M. Taoka, H. Donai, N. Takahashi, T. Isobe, and T. Yamauchi, "Molecular constituents of the postsynaptic density fraction revealed by proteomic analysis using multidimensional liquid chromatography-tandem mass spectrometry," J Neurochem, vol. 88, no. 3, pp. 759-68, 2004. 73,75

[235] N. S. Abul-Husn, I. Bushlin, J. A. Moron, S. L. Jenkins, G. Dolios, R. Wang, R. Iyengar, A. Ma'ayan, and L. A. Devi, "Systems approach to explore components and interactions in the presynapse," Proteomics, vol. 9, no. 12, pp. 3303-15, 2009. 74, 79

[236] C. Geumann, M. Gronborg, M. Hellwig, H. Martens, and R. Jahn, "A sandwich enzymelinked immunosorbent assay for the quantification of insoluble membrane and scaffold proteins," Anal Biochem, vol. 402, no. 2, pp. 161-9, 2010. 75

[237] O. Berninghausen, M. A. Rahman, J. P. Silva, B. Davletov, C. Hopkins, and Y. A. Ushkaryov, "Neurexin ibeta and neuroligin are localized on opposite membranes in mature central synapses," J Neurochem, vol. 103, no. 5, pp. 1855-63, 2007. 75, 81

[238] P. Scheiffele, J. Fan, J. Choih, R. Fetter, and T. Serafini, "Neuroligin expressed in nonneuronal cells triggers presynaptic development in contacting axons," Cell, vol. 101, no. 6, pp. 657-69, 2000. 76

[239] E. R. Graf, X. Zhang, S. X. Jin, M. W. Linhoff, and A. M. Craig, "Neurexins induce differentiation of gaba and glutamate postsynaptic specializations via neuroligins," Cell, vol. 119, no. 7, pp. 1013-26, 2004. 76

[240] M. B. Dalva, M. A. Takasu, M. Z. Lin, S. M. Shamah, L. Hu, N. W. Gale, and M. E. Greenberg, "Ephb receptors interact with nmda receptors and regulate excitatory synapse formation," Cell, vol. 103, no. 6, pp. 945-56, 2000. 76

[241] S. Das, A. D. Bosley, X. Ye, K. C. Chan, I. Chu, J. E. Green, H. J. Issaq, T. D. Veenstra, and T. Andresson, "Comparison of strong cation exchange and sds-page fractionation for analysis of multiprotein complexes," J Proteome Res, vol. 9, no. 12, pp. 6696-704, 2010. 76

[242] M. R. Wilkins, E. Gasteiger, J. C. Sanchez, A. Bairoch, and D. F. Hochstrasser, "Twodimensional gel electrophoresis for proteome projects: the effects of protein hydrophobicity and copy number," Electrophoresis, vol. 19, no. 8-9, pp. 1501-5, 1998. 77 
[243] K. W. Li, M. P. Hornshaw, R. C. Van Der Schors, R. Watson, S. Tate, B. Casetta, C. R. Jimenez, Y. Gouwenberg, E. D. Gundelfinger, K. H. Smalla, and A. B. Smit, "Proteomics analysis of rat brain postsynaptic density. implications of the diverse protein functional groups for the integration of synaptic physiology," J Biol Chem, vol. 279, no. 2, pp. 9871002, 2004. 77

[244] J. Hartinger, K. Stenius, D. Hogemann, and R. Jahn, "16-bac/sds-page: a twodimensional gel electrophoresis system suitable for the separation of integral membrane proteins," Anal Biochem, vol. 240, no. 1, pp. 126-33, 1996. 77

[245] R. P. Zahedi, C. Meisinger, and A. Sickmann, "Two-dimensional benzyldimethyl-nhexadecylammonium chloride/sds-page for membrane proteomics," Proteomics, vol. 5, no. 14 , pp. 3581-8, 2005. 77

[246] J. Stevens, S. M., A. D. Zharikova, and L. Prokai, "Proteomic analysis of the synaptic plasma membrane fraction isolated from rat forebrain," Brain Res Mol Brain Res, vol. 117, no. 2, pp. 116-28, 2003. 77

[247] L. J. Foster, C. L. de Hoog, Y. Zhang, X. Xie, V. K. Mootha, and M. Mann, "A mammalian organelle map by protein correlation profiling," Cell, vol. 125, no. 1, pp. 187-99, 2006. 78

[248] J. S. Kang, J. H. Tian, P. Y. Pan, P. Zald, C. Li, C. Deng, and Z. H. Sheng, "Docking of axonal mitochondria by syntaphilin controls their mobility and affects short-term facilitation," Cell, vol. 132, no. 1, pp. 137-48, 2008.78

[249] Y. M. Chen, C. Gerwin, and Z. H. Sheng, "Dynein light chain lc8 regulates syntaphilinmediated mitochondrial docking in axons," J Neurosci, vol. 29, no. 30, pp. 9429-38, 2009. 78

[250] G. A. Perkins, J. Tjong, J. M. Brown, P. H. Poquiz, R. T. Scott, D. R. Kolson, M. H. Ellisman, and G. A. Spirou, "The micro-architecture of mitochondria at active zones: electron tomography reveals novel anchoring scaffolds and cristae structured for highrate metabolism," J Neurosci, vol. 30, no. 3, pp. 1015-26, 2010. 78

[251] C. W. Lee and H. B. Peng, "The function of mitochondria in presynaptic development at the neuromuscular junction," Mol Biol Cell, vol. 19, no. 1, pp. 150-8, 2008. 78

[252] P. Verstreken, C. V. Ly, K. J. Venken, T. W. Koh, Y. Zhou, and H. J. Bellen, "Synaptic mitochondria are critical for mobilization of reserve pool vesicles at drosophila neuromuscular junctions," Neuron, vol. 47, no. 3, pp. 365-78, 2005. 78 
[253] R. Chittajallu, S. P. Braithwaite, V. R. Clarke, and J. M. Henley, "Kainate receptors: subunits, synaptic localization and function," Trends Pharmacol Sci, vol. 20, no. 1, pp. 2635, 1999. 80

[254] P. S. Pinheiro, D. Perrais, F. Coussen, J. Barhanin, B. Bettler, J. R. Mann, J. O. Malva, S. F. Heinemann, and C. Mulle, "Glur7 is an essential subunit of presynaptic kainate autoreceptors at hippocampal mossy fiber synapses," Proc Natl Acad Sci U S A, vol. 104, no. 29 , pp. $12181-6,2007.80$

[255] M. E. Tremblay, M. Riad, D. Bouvier, K. K. Murai, E. B. Pasquale, L. Descarries, and G. Doucet, "Localization of epha4 in axon terminals and dendritic spines of adult rat hippocampus," J Comp Neurol, vol. 501, no. 5, pp. 691-702, 2007. 80

[256] A. Chattopadhyay and E. London, "Fluorimetric determination of critical micelle concentration avoiding interference from detergent charge," Anal Biochem, vol. 139, no. 2, pp. 408-12, 1984. 81

[257] J. Almarza, L. Rincon, A. Bahsas, and F. Brito, "Molecular mechanism for the denaturation of proteins by urea," Biochemistry, vol. 48, no. 32, pp. 7608-13, 2009. 81

[258] A. G. Leenders, F. H. Lopes da Silva, W. E. Ghijsen, and M. Verhage, "Rab3a is involved in transport of synaptic vesicles to the active zone in mouse brain nerve terminals," Mol Biol Cell, vol. 12, no. 10, pp. 3095-102, 2001. 81

[259] S. O. Rizzoli and W. J. Betz, "The structural organization of the readily releasable pool of synaptic vesicles," Science, vol. 303, no. 5666, pp. 2037-9, 2004. 82

[260] R. M. Weimer, E. O. Gracheva, O. Meyrignac, K. G. Miller, J. E. Richmond, and J. L. Bessereau, "Unc-13 and unc-10/rim localize synaptic vesicles to specific membrane domains," J Neurosci, vol. 26, no. 31, pp. 8040-7, 2006. 82

[261] F. E. Zilly, J. B. Sorensen, R. Jahn, and T. Lang, "Munc18-bound syntaxin readily forms snare complexes with synaptobrevin in native plasma membranes," PLoS Biol, vol. 4, no. 10 , p. e330, 2006. 82,83

[262] P. Burkhardt, D. A. Hattendorf, W. I. Weis, and D. Fasshauer, "Munc18a controls snare assembly through its interaction with the syntaxin n-peptide," $E M B O J$, vol. 27, no. 7, pp. 923-33, 2008. 82 
[263] C. N. Medine, C. Rickman, L. H. Chamberlain, and R. R. Duncan, "Munc18-1 prevents the formation of ectopic snare complexes in living cells," J Cell Sci, vol. 120, no. Pt 24, pp. $4407-15,2007$. 82

[264] D. Fasshauer, W. Antonin, V. Subramaniam, and R. Jahn, "Snare assembly and disassembly exhibit a pronounced hysteresis," Nat Struct Biol, vol. 9, no. 2, pp. 144-51, 2002. 83

[265] M. Morales, M. A. Colicos, and Y. Goda, "Actin-dependent regulation of neurotransmitter release at central synapses," Neuron, vol. 27, no. 3, pp. 539-50, 2000. 83

[266] S. Sankaranarayanan, P. P. Atluri, and T. A. Ryan, "Actin has a molecular scaffolding, not propulsive, role in presynaptic function," Nat Neurosci, vol. 6, no. 2, pp. 127-35, 2003. 83

[267] T. R. Mahoney, Q. Liu, T. Itoh, S. Luo, G. Hadwiger, R. Vincent, Z. W. Wang, M. Fukuda, and M. L. Nonet, "Regulation of synaptic transmission by rab-3 and rab27 in caenorhabditis elegans," Mol Biol Cell, vol. 17, no. 6, pp. 2617-25, 2006. 83

[268] A. C. Figueiredo, C. Wasmeier, A. K. Tarafder, J. S. Ramalho, R. A. Baron, and M. C. Seabra, "Rab3gep is the non-redundant guanine nucleotide exchange factor for rab27a in melanocytes," J Biol Chem, vol. 283, no. 34, pp. 23209-16, 2008. 83

[269] L. M. Chavas, S. Torii, H. Kamikubo, M. Kawasaki, K. Ihara, R. Kato, M. Kataoka, T. Izumi, and S. Wakatsuki, "Structure of the small gtpase rab27b shows an unexpected swapped dimer," Acta Crystallogr D Biol Crystallogr, vol. 63, no. Pt 7, pp. 769-79, 2007. 83

[270] L. Deng, P. S. Kaeser, W. Xu, and T. C. Sudhof, "Rim proteins activate vesicle priming by reversing autoinhibitory homodimerization of munc13," Neuron, vol. 69, no. 2, pp. 317-31, 2011. 83 


\section{Acknowledgements}

I want to thank Prof. Reinhard Jahn for giving me the opportunity to work in his group. Reinhard, thank you for your support, the ideas you have shared with me and all the motivation throughout the last years. I really enjoyed working in your lab and I am honored that I could even do experiments with you.

I am deeply grateful to Dr. John Chua for his tremendous support throughout my $\mathrm{PhD}$. John, you have been an extraordinary teacher and a great friend to me. Thank you for all the scientific skills you taught me, for all the lively discussions and for guiding me through my $\mathrm{PhD}$. You always knew the right things to say: You motivated me when I was frustrated and you brought me back to earth when I was hyper-active.

I thank Prof. Ficner and Prof. Thumm for being part of my thesis committee and for their comments and ideas during the committee meetings.

I would like to thank my bench- and office mate Dr. Hans Dieter Schmitt for his support during the years, although he had to bear a large number of intensively smelling fruit teas, emotional outbursts and temporary attacks of complete insanity. Dieter, you are a walking scientific wikipedia paired with McGyver skills and equipped with endless sugar supplies. Believe me, this is a compliment! You are amazing.

I want to thank Maria Druminski for her technical help and personal commitment. Life is not fair.

I would also like to thank Dr. Mads Gronborg for his help with the mass spectrometry part of this thesis. Mads, it was a great pleasure working with you. You are my favorite danish and the only one I know who can wear pullovers with horizontal stripes. 
I thank Prof. Henning Urlaub for his support and for integrating me into his group. I really enjoyed the summer school last year.

I would also like to thank Dr. Dietmar Riedel and Dr. Dirk Wenzel for the electron microscopy pictures in this thesis.

Many thanks to the people in the animal facility in tower 6 .

Doing a $\mathrm{PhD}$ requires endless working hours, therefore I want to thank my present and former colleagues from the department of Neurobiology for the great working atmosphere. I am especially grateful to Dr. Gottfried Mieskes, for always having an open door for the needs and problems of the people in the lab and for having the skills to solve almost every one of these problems.

During the time in the lab I met people that have become dear frineds. Anna, Caro, Julia, Saskia, Ricarda, Constanze, Ulf and Sabrina, thank you for all the fun times, the uncountable discussions by one or another drink, the tons of sweat shared during running, climbing, swimming or fitness classes and for your friendship over the years.

Finally I want to thank my family for their support and love. 


\title{
Curriculum Vitae
}

\section{Personal DATA}

Anne Janina Boyken

Address Lotzestr.2

Goettingen, Germany

Email jboyken@gwdg.de

Date of Birth 02. August 1983

\section{EDUCATION}

\author{
2008-2011 | Max-Planck-Institute for Biophysical Chemistry \\ $\mathrm{PhD}$ in the Department of Neurobiology \\ Thesis: Molecular profiling of presynaptic docking sites \\ Georg-August-University Goettingen \\ Member of the doctoral program "Biomolecules: Structure-Function-Dynamics" \\ Goettingen Graduate School for Neurosciences and Molecular Biosciences (GGNB). \\ 2006-2007 Sanofi-Aventis Deutschland GmbH \\ Diploma in the Department for Metabolic Disorders \\ Thesis: Enzymatic and biophysical investigation of glucose metabolism \\ 2002-2007 University of Greifswald, Germany \\ Biochemistry (Diploma)

\section{TEACHING} \\ 2011 \\ 2008-2010 \\ Tutor of lectures \\ "Protein Sorting and Processing" in the International MSc/PhD Pro- \\ grams "Molecular Biology" and "Neurosciences"

\section{Tutor of method courses} \\ "Subcellular Fractionation and Centrifugation" within the International \\ MSc/PhD Program "Molecular Biology" \\ "Co-immunoprecipitation as a technique to study protein-protein inter- \\ actions" \\ within the Goettingen Graduate School for Neurosciences and Molecular \\ Biosciences (GGNB) \\ 2009 Supervisor of Labrotations \\ Momchil Ninov and Jonathan Melin within the the International \\ MSc/PhD Program "Molecular Biology"
}




\section{Conferences}

2011 "Synapses: From Molecules to Circuits and Behavior"

Cold Spring Harbor Laboratory Meeting

Cold Spring Harbor, USA (poster)

2010 "Horizons in Molecular Biology"

Goettingen, Germany (poster)

2010 "Proteomics Basics- High Throughput Data Analysis and Statistics" 4th European Summer School, Brixen, South Italy (poster)

\section{Publications}

2010 Chua JJ, Kindler S, Boyken J, Jahn R

The architecture of an excitatory synapse

J Cell Sci. 2010 Mar 15;123(Pt 6):819-23. Review

2010 Pavlos NJ, Gronborg M, Riedel D, Chua JJ, Boyken J,

Kloepper TH, Urlaub H, Rizzoli SO, Jahn R.

Quantitative analysis of synaptic vesicle Rabs uncovers distinct yet overlapping roles for Rab3a and Rab27b in Ca2+-triggered exocytosis

J Neurosci. 2010 Oct 6;30(40):13441-53 
GI number \# identified in protein name

biological

replicates biological biological biological average standard protein localization/function replicate replicate replicate enrichment deviation

\begin{tabular}{|c|c|c|c|c|c|c|}
\hline gi|11693170 & 3 2-oxoglutarate carrier & SPM & 4.5 & 7.5 & 6.0 & 2.1 mitochondria \\
\hline gi| 55742813 & 3 3-hydroxybutyrate dehydrogenase, type 1 & 4.3 & 5.2 & 9.4 & 6.3 & 2.7 mitochondria \\
\hline gi| 83977457 & 2 3-hydroxyisobutyrate dehydrogenase & & SPM & SPM & SPM & mitochondria \\
\hline gi|20304123 & 2 3-mercaptopyruvate sulfurtransferase & & 5.2 & 7.4 & 6.3 & 1.5 mitochondria \\
\hline gi|189181716 & 3 3-oxoacid CoA transferase 1 & SPM & SPM & 8.8 & SPM & mitochondria \\
\hline gi|13591900 & 3 4-aminobutyrate aminotransferase & 4.7 & 7.4 & 7.0 & 6.4 & 1.5 mitochondria \\
\hline gi|209969744 & 3 4-nitrophenylphosphatase domain and non-neuronal SNAP25-like protein homolog 1 & 5.9 & 7.3 & 7.9 & 7.0 & 1.1 mitochondria \\
\hline gi|109478763 & $36.8 \mathrm{kDa}$ mitochondrial proteolipid & SPM & SPM & SPM & SPM & mitochondria \\
\hline gi|8392836 & 3 acetyl-Coenzyme $A$ acetyltransferase 1 & 8.0 & 6.8 & 5.8 & 6.9 & 1.1 mitochondria \\
\hline gi|40538860 & 3 aconitase 2 , mitochondrial & 5.1 & 4.7 & 5.4 & 5.1 & 0.4 mitochondria \\
\hline gi|18543341 & 3 acyl-CoA synthetase long-chain family member 6 & 8.3 & 5.9 & 2.3 & 5.5 & 3.0 mitochondria \\
\hline gi|62078649 & 3 acyl-CoA thioesterase 9 & SPM & 7.8 & 4.1 & 5.9 & 2.6 mitochondria \\
\hline gi|197313734 & 2 acyl-Coenzyme $A$ dehydrogenase family, member 9 & & SPM & SPM & SPM & mitochondria \\
\hline gi|6978435 & 2 acyl-Coenzyme $A$ dehydrogenase, very long chain & & SPM & SPM & SPM & mitochondria \\
\hline gi|188595700 & 2 acylglycerol kinase & & 9.3 & 7.8 & 8.6 & 1.1 mitochondria \\
\hline gi|58865518 & 3 aldehyde dehydrogenase $1 \mathrm{~B} 1$ & SPM & SPM & SPM & SPM & mitochondria \\
\hline gi|109475727 & 2 aldehyde dehydrogenase 4 family, member A1 & & SPM & 6.7 & 6.7 & mitochondria \\
\hline gi|34933197 & 3 Amine oxidase [flavin-containing] A (Monoamine oxidase type A) (MAO-A) & 6.3 & 6.8 & 6.4 & 6.5 & 0.2 mitochondria \\
\hline gi|110189667 & 3 ATP synthase F0 subunit 8 & 4.1 & SPM & SPM & 4.1 & mitochondria \\
\hline gi|19705465 & 3 ATP synthase, $H+$ transporting, mitochondrial F0 complex, subunit B1 & 4.5 & 5.6 & 2.2 & 4.1 & 1.7 mitochondria \\
\hline gi|9506411 & 3 ATP synthase, $\mathrm{H}+$ transporting, mitochondrial F0 complex, subunit d & 5.7 & 2.7 & 3.6 & 4.0 & 1.5 mitochondria \\
\hline gi|17978459 & 3 ATP synthase, $\mathrm{H}+$ transporting, mitochondrial F0 complex, subunit E & 5.7 & SPM & 4.9 & 5.3 & 0.6 mitochondria \\
\hline gi|109495163 & 3 ATP synthase, $H+$ transporting, mitochondrial $F 0$ complex, subunit $f$, isoform 2 & 32.7 & 8.6 & SPM & SPM & mitochondria \\
\hline gi|16758388 & 3 ATP synthase, $\mathrm{H}+$ transporting, mitochondrial F0 complex, subunit F6 & SPM & SPM & SPM & SPM & mitochondria \\
\hline gi|47058994 & 3 ATP synthase, $\mathrm{H}+$ transporting, mitochondrial F0 complex, subunit $\mathrm{G}$ & SPM & SPM & 6.0 & 6.0 & mitochondria \\
\hline gi|40538742 & 3 ATP synthase, $\mathrm{H}+$ transporting, mitochondrial $\mathrm{F} 1$ complex, alpha subunit 1 & 6.1 & 6.1 & 7.9 & 6.7 & 1.0 mitochondria \\
\hline $\mathrm{gi} \mid 20806153$ & 3 ATP synthase, $\mathrm{H}+$ transporting, mitochondrial F1 complex, delta subunit & SPM & SPM & SPM & SPM & mitochondria \\
\hline gi|20806139 & 3 ATP synthase, $\mathrm{H}+$ transporting, mitochondrial $\mathrm{F} 1$ complex, epsilon subunit & SPM & 5.8 & 9.3 & 7.6 & 2.5 mitochondria \\
\hline gi|39930503 & 3 ATP synthase, $\mathrm{H}+$ transporting, mitochondrial $\mathrm{F} 1$ complex, gamma subunit & 2.9 & 3.3 & 6.7 & 4.3 & 2.1 mitochondria \\
\hline gi|77917538 & 2 ATPase family, AAA domain containing 3A & & SPM & SPM & SPM & mitochondria \\
\hline gi|77917528 & 3 ATPase inhibitory factor 1 & 4.4 & 5.6 & 4.6 & 4.9 & 0.6 mitochondria \\
\hline gi|47058990 & 3 ATP-binding cassette, sub-family B, member 7, mitochondrial precursor & SPM & SPM & SPM & SPM & mitochondria \\
\hline gi|157822275 & 3 AU RNA binding protein/enoyl-Coenzyme A hydratase & 9.4 & 6.1 & SPM & 7.7 & 2.3 mitochondria \\
\hline gi|56090628 & 3 BCS1-like & SPM & SPM & SPM & SPM & mitochondria \\
\hline gi|81295385 & 3 biphenyl hydrolase-like (serine hydrolase) & SPM & SPM & SPM & SPM & mitochondria \\
\hline gi|117647218 & 3 brain protein 44 & 8.1 & 4.3 & 6.6 & 6.3 & 2.0 mitochondria \\
\hline gi|19424244 & 3 brain protein 44 -like & SPM & 7.1 & SPM & SPM & mitochondria \\
\hline gi|62646841 & 3 Calcium-binding mitochondrial carrier protein Aralar2 & SPM & 2.6 & 8.7 & 5.6 & 4.3 mitochondria \\
\hline
\end{tabular}


GI number \# identified in protein name biological gi|157824004 gi| 18543177 gi| 157817027 gi||157819769 gi| 48675371 gi|60678254 gi|110189675 gi| 61557037 gi|20302049 gi|110189665 gi| 110189665 gi| 110189718 gi|110189669 gi| 8393180 gi| 16758362 gi|157821821 gi|65301490 gi| 24233541 gi| 77736544 gi| 109465447 gi| 160333459 $\mathrm{gi} \mid 11968072$ gi) 197927439 gi| 197927439 gi| 6978725 gi| 194473626 gi| 109510612 gi| 19424210 gi|58865478 gi| 40786469 gi| 78365255 gi|195927000 gi| 57527204 gi| 52138635 gi|157821933 gi| 157821933 gil 151948422 gi|51948422 gi| 157786896 gi| 16758100 gi|158186722

\section{replicates}

3 citrate synthase

2 cytochrome b

3 cytochrome c-1

3 dapit protein

2 es1 protein

3 CDGSH iron sulfur domain 1

3 coiled-coil-helix-coiled-coil-helix domain containing 3

2 coiled-coil-helix-coiled-coil-helix domain containing 6

3 complement component 1 , q subcomponent binding protein

3 creatine kinase, mitochondrial 1 , ubiquitous

2 cytochrome b5 reductase 1

2 cytochrome b5 reductase 3

3 cytochrome coxidase subunit 1

cytochrome coxidase subunit II

3 cytochrome coxidase subunit IV isoform 1

3 cytochrome coxidase subunit IV $\mathrm{V}$

3 cytochrome c oxidase subunit $\mathrm{Vb}$

3 cytochrome coxidase subunit VII

3 cytochrome c oxidase, subunit $V$

3 cytochrome c oxidase, subunit Vla, polypeptide 1

3 cytochrome c oxidase, subunit VIb polypeptide 1

3 cytochrome $c$ oxidase, subunit VIc

3 cytochrome c oxidase, subunit VIla 2

3 cytochrome c oxidase, subunit VIIc

3 cytochrome c, somatic

Cytochrome c-type heme lyase (CCHL) (Holocytochrome c-type synthase)

3 death associated protein 3

3 dihydrolipoamide dehydrogenase

3 dihydrolipoamide S-acetyltransferase

3 dihydrolipoamide S-succinyltransferase

3 electron-transfer-flavoprotein, alpha polypeptide

2 electron-transferring-flavoprotein dehydrogenase

3 endo/exonuclease $\left(5^{\sim}-3^{\sim}\right)$, endonuclease $G$-like precursor

2 enoyl Coenzyme A hydratase, short chain, 1 , mitochondrial

2 fission 1 (mitochondrial outer membrane) homolos

2 fractured callus expressed transcript 1

3 fumarate hydratase 1 biological biological biological average standard protein localization/function replicate replicate replicate enrichment deviation

$\begin{array}{rrrr}8.4 & 7.0 & 5.6 & 7.0 \\ 5.6 & 7.0 & 5.9 & 6.2 \\ 4.4 & 8.0 & 5.1 & 5.8 \\ \text { SPM } & 2.0 & & \\ \text { SPM } & \text { SPM } & \text { SPM } & \text { SPM } \\ 6.9 & 7.1 & 6.6 & 6.9 \\ & \text { SPM } & 8.9 & 8.9 \\ & \text { SPM } & \text { SPM } & \text { SPM } \\ & 2.5 & 7.6 & 5.1 \\ \text { SPM } & \text { SPM } & \text { SPM } & \text { SPM } \\ \text { SPM } & \text { SPM } & \text { SPM } & \text { SPM } \\ \text { SPM } & \text { SPM } & \text { SPM } & \text { SPM } \\ 3.0 & 6.4 & 1.7 & 3.7 \\ 2.2 & 3.2 & 10.4 & 5.3 \\ 2.6 & \text { SPM } & 5.3 & 4.0 \\ \text { SPM } & 7.3 & 7.4 & 7.3 \\ 1.8 & 5.1 & 3.6 & 3.5 \\ \text { SPM } & \text { SPM } & \text { SPM } & \text { SPM } \\ & 6.2 & \text { SPM } & 6.2 \\ \text { SPM } & 9.6 & 5.8 & 7.7 \\ \text { SPM } & \text { SPM } & \text { SPM } & \text { SPM } \\ \text { SPM } & \text { SPM } & \text { SPM } & \text { SPM } \\ \text { SPM } & 5.0 & \text { SPM } & \text { SPM } \\ \text { SPM } & 4.7 & \text { SPM } & \text { SPM } \\ & 10.4 & \text { SPM } & \text { SPM } \\ \text { SPM } & 7.1 & \text { SPM } & \text { SPM } \\ \text { SPM } & \text { SPM } & \text { SPM } & \text { SPM } \\ 9.8 & 5.3 & 6.8 & 7.3 \\ 7.4 & 4.5 & 6.6 & 6.2 \\ \text { SPM } & 6.9 & 4.2 & 5.5 \\ \text { SPM } & \text { SPM } & \text { SPM } & \text { SPM } \\ & \text { SPM } & \text { SPM } & \text { SPM } \\ \text { SPM } & \text { SPM } & \text { SPM } & \text { SPM } \\ & 5.6 & \text { SPM } & \text { SPM } \\ & 5.8 & 11.0 & 8.4 \\ & \text { SPM } & 5.1 & \text { SPM } \\ 7.5 & \text { SPM } & \text { SPM } & \text { SPM } \\ & 8.5 & 7.2 & 7.7 \\ & & & \end{array}$

0.8 mitochondria

1.9 mitochondria

mitochondria

mitochondria

0.3 mitochondria

mitochondria

3.6 mitochondria

mitochondria

mitochondria

mitochondria

2.4 mitochondria

4.5 mitochondria

1.9 mitochondria

0.1 mitochondria

.7 mitochondria

mitochondria

2.7 mitochondria

mitochondria

mitochondria

mitochondria

mitochondria

mitochondria

mitochondria

mitochondria

2.3 mitochondria

1.5 mitochondria

1.9 mitochondria

mitochondria

mitochondria

mitochondria

mitochondria

3.7 mitochondria

mitochondria

mitochondria
0.7 mitochondria 
GI number \# identified in protein name biological biological biological biological average standard protein localization/function replicate replicate replicate enrichment deviation

\begin{tabular}{|c|c|c|c|c|c|c|}
\hline gi|68163417 & 2 fumarylacetoacetate hydrolase domain containing 1 & & SPM & SPM & SPM & mitochondria \\
\hline gi|62945328 & 3 glioblastoma amplified sequence & SPM & 3.7 & 6.7 & 5.2 & 2.1 mitochondria \\
\hline gi|6980956 & 3 glutamate dehydrogenase 1 & 5.9 & 3.5 & 4.4 & 4.6 & 1.2 mitochondria \\
\hline gi|6980972 & 3 glutamate oxaloacetate transaminase 2 & 8.6 & 7.1 & 7.0 & 7.6 & 0.9 mitochondria \\
\hline gi|158303294 & 3 glutaminase isoform a & 7.1 & 4.1 & 6.3 & 5.8 & 1.5 mitochondria \\
\hline gi|6980978 & 3 glycerol-3-phosphate dehydrogenase 2 & 7.5 & 3.9 & 6.2 & 5.9 & 1.8 mitochondria \\
\hline gi|13324704 & 2 GrpE-like 1 , mitochondrial & & 2.5 & 4.1 & 3.3 & 1.1 mitochondria \\
\hline gi|6981052 & 3 heat shock $10 \mathrm{kDa}$ protein 1 & 7.0 & SPM & 24.4 & 15.7 & 12.3 mitochondria \\
\hline gi|206597443 & 3 heat shock protein 1 (chaperonin) & 4.8 & 7.4 & 6.0 & 6.1 & 1.3 mitochondria \\
\hline gi|154816168 & 3 heat shock protein 9 & 6.7 & 5.0 & 7.6 & 6.5 & 1.3 mitochondria \\
\hline gi|6981022 & 3 hexokinase 1 & 5.5 & 6.9 & 7.6 & 6.7 & 1.1 mitochondria \\
\hline gi|38454320 & 3 hormone-regulated proliferation associated protein 20 & SPM & 9.5 & SPM & SPM & mitochondria \\
\hline gi|109487466 & 3 hypothetical protein (NADH:Ubiquinone oxioreductase, $42 \mathrm{kDa}(\mathrm{NDUO42)})$ & 8.6 & 4.9 & SPM & 6.7 & 2.7 mitochondria \\
\hline gi|77917546 & 3 inner membrane protein, mitochondrial & 9.7 & 5.4 & 5.8 & 7.0 & 2.4 mitochondria \\
\hline gi|109467571 & 2 inorganic pyrophosphatase 2 & & 3.5 & SPM & & mitochondria \\
\hline gi|62079055 & 2 isocitrate dehydrogenase $2(\mathrm{NADP}+)$, mitochondrial & SPM & 0.0 & 8.9 & 4.5 & 6.3 mitochondria \\
\hline gi|16758446 & 3 isocitrate dehydrogenase $3(\mathrm{NAD}+)$ alpha & 8.0 & 6.9 & 4.2 & 6.3 & 2.0 mitochondria \\
\hline gi|55926203 & 3 isocitrate dehydrogenase 3 , beta subunit & 6.0 & 6.8 & 8.8 & 7.2 & 1.4 mitochondria \\
\hline gi|54020666 & 3 isocitrate dehydrogenase 3 , gamma & 8.9 & 8.6 & 5.2 & 7.6 & 2.1 mitochondria \\
\hline gi|54400736 & 3 leucine zipper-EF-hand containing transmembrane protein 1 & 2.4 & 0.7 & 0.9 & 1.4 & 0.9 mitochondria \\
\hline gi|19173766 & 2 lon peptidase 1 , mitochondrial & SPM & 0.0 & SPM & SPM & mitochondria \\
\hline gi|42476181 & 3 malate dehydrogenase, mitochondrial & 5.9 & 8.9 & 5.9 & 6.9 & 1.8 mitochondria \\
\hline gi|157817153 & 3 malic enzyme $3, \mathrm{NADP}(+)$-dependent, mitochondrial & 5.4 & SPM & 9.5 & 7.5 & 2.9 mitochondria \\
\hline gi|56605654 & 2 metaxin 2 & & 5.1 & 1.9 & 3.5 & 2.3 mitochondria \\
\hline gi|62660299 & 3 microsomal glutathione S-transferase 3 & SPM & SPM & SPM & SPM & mitochondria \\
\hline gi|34875107 & 2 mitchondrial ribosomal protein $\mathrm{S7}$ & & SPM & SPM & SPM & mitochondria \\
\hline gi|54792127 & 3 mitochondrial ATP synthase beta subunit & 7.2 & 5.8 & 8.2 & 7.1 & 1.2 mitochondria \\
\hline gi|20302061 & 3 mitochondrial ATP synthase, 0 subunit & 4.8 & 8.1 & 4.7 & 5.9 & 1.9 mitochondria \\
\hline gi|197313797 & 3 mitochondrial carrier homolog 1 & SPM & SPM & 5.2 & SPM & mitochondria \\
\hline gi|158819029 & 3 mitochondrial carrier homolog 2 & 5.2 & SPM & 7.4 & 6.3 & 1.6 mitochondria \\
\hline gi|27695671 & 3 mitochondrial carrier triple repeat 1 & & SPM & SPM & SPM & mitochondria \\
\hline gi|109472570 & 2 Mitochondrial glutamate carrier 2 & SPM & 0.0 & 4.1 & 2.0 & 2.9 mitochondria \\
\hline gi|55741522 & 2 mitochondrial protein $18 \mathrm{kDa}$ & & SPM & SPM & SPM & mitochondria \\
\hline gi|57164123 & 2 mitochondrial ribosomal protein $\mathrm{L} 38$ & & 1.3 & SPM & & mitochondria \\
\hline gi|157786906 & 2 mitochondrial ribosomal protein S17 & & SPM & SPM & SPM & mitochondria \\
\hline gi|157824010 & 2 mitochondrial ribosomal protein $\mathrm{S} 31$ & & SPM & SPM & SPM & mitochondria \\
\hline gi|109464325 & 3 mitochondrial ribosomal protein S36 & SPM & SPM & 5.4 & SPM & mitochondria \\
\hline gi|148747393 & 2 mitochondrial trifunctional protein, alpha subunit & & 6.5 & 7.3 & 6.9 & 0.5 mitochondria \\
\hline
\end{tabular}


Gl number \# identified in protein name biological

\begin{tabular}{|c|c|}
\hline$\overline{\text { gi||148747472 }}$ & 2 mitofusin 2 \\
\hline gi|6981260 & 3 NADH dehydrogenase (ubiquinone) 1 alpha subcomplex 5 \\
\hline gi|47058992 & 3 NADH dehydrogenase (ubiquinone) 1 alpha subcomplex 11 \\
\hline gi|157818537 & 2 NADH dehydrogenase (ubiquinone) 1 alpha subcomplex, 1 \\
\hline gi|164565371 & 3 NADH dehydrogenase (ubiquinone) 1 alpha subcomplex, 12 \\
\hline gi|27718097 & 3 NADH dehydrogenase (ubiquinone) 1 alpha subcomplex, 13 \\
\hline gi|157817861 & 3 NADH dehydrogenase (ubiquinone) 1 alpha subcomplex, 2 \\
\hline gi|189085365 & 3 NADH dehydrogenase (ubiquinone) 1 alpha subcomplex, 4 \\
\hline gi|194473636 & 3 NADH dehydrogenase (ubiquinone) 1 alpha subcomplex, 6 (B14) \\
\hline gi|157824069 & $2 \mathrm{NADH}$ dehydrogenase (ubiquinone) 1 alpha subcomplex, 7 (B14.5a) \\
\hline gi|114145517 & 3 NADH dehydrogenase (ubiquinone) 1 alpha subcomplex, 8 \\
\hline gi|198278533 & $3 \mathrm{NADH}$ dehydrogenase (ubiquinone) 1 alpha subcomplex, 9 \\
\hline gi|82617686 & 2 NADH dehydrogenase (ubiquinone) 1 beta subcomplex 4 \\
\hline gi|157822261 & 3 NADH dehydrogenase (ubiquinone) 1 beta subcomplex 8 \\
\hline gi|157822851 & $3 \mathrm{NADH}$ dehydrogenase (ubiquinone) 1 beta subcomplex, 11 \\
\hline gi|157823387 & 3 NADH dehydrogenase (ubiquinone) 1 beta subcomplex, 5 \\
\hline gi|157820465 & $3 \mathrm{NADH}$ dehydrogenase (ubiquinone) 1 beta subcomplex, 6 \\
\hline gi|157823197 & $2 \mathrm{NADH}$ dehydrogenase (ubiquinone) 1 beta subcomplex, 7 \\
\hline gi|187937028 & 3 NADH dehydrogenase (ubiquinone) 1 beta subcomplex, 9 \\
\hline gi|157820787 & $3 \mathrm{NADH}$ dehydrogenase (ubiquinone) 1 , alpha/beta subcomplex, 1 \\
\hline gi|57164133 & 3 NADH dehydrogenase (ubiquinone) 1 , subcomplex unknown, 2 \\
\hline gi|53850628 & $3 \mathrm{NADH}$ dehydrogenase (ubiquinone) Fe-S protein 1, 75kDa \\
\hline gi|58865384 & $3 \mathrm{NADH}$ dehydrogenase (ubiquinone) Fe-S protein 2 \\
\hline gi|157817227 & $3 \mathrm{NADH}$ dehydrogenase (ubiquinone) Fe-S protein 3 \\
\hline gi|68341995 & $3 \mathrm{NADH}$ dehydrogenase (ubiquinone) Fe-S protein 4 \\
\hline gi|72086149 & 3 NADH dehydrogenase (ubiquinone) Fe-S protein $5 b$ \\
\hline gi|109460535 & 3 NADH dehydrogenase (ubiquinone) Fe-S protein 6 \\
\hline gi|56606108 & $3 \mathrm{NADH}$ dehydrogenase (ubiquinone) Fe-S protein 7 \\
\hline gi|157821497 & 3 NADH dehydrogenase (ubiquinone) Fe-S protein 8 \\
\hline gi|55741424 & $3 \mathrm{NADH}$ dehydrogenase (ubiquinone) flavoprotein $1,51 \mathrm{kDa}$ \\
\hline gi|51092268 & $3 \mathrm{NADH}$ dehydrogenase (ubiquinone) flavoprotein 2 \\
\hline gi|162287192 & $2 \mathrm{NADH}$ dehydrogenase (ubiquinone) flavoprotein 3 -like isoform 1 \\
\hline gi|110189663 & $3 \mathrm{NADH}$ dehydrogenase subunit 1 \\
\hline gi|110189672 & $3 \mathrm{NADH}$ dehydrogenase subunit 4 \\
\hline gi|110189673 & $3 \mathrm{NADH}$ dehydrogenase subunit 5 \\
\hline gi|109467413 & 3 NADH-ubiquinone oxidoreductase B9 subunit (Complex I-B9) (CI-B9 \\
\hline gi|109490343 & 3 NADH-ubiquinone oxidoreductase PDSW subunit \\
\hline gi|11968102 & 2 ornithine aminotransferase \\
\hline
\end{tabular}

biological biological biological average standard protein localization/function replicate replicate replicate enrichment deviation

$\begin{array}{rrrrr}\# 1 & \# 2 & \# 3 & & \\ & & & & \\ 7.4 & \text { SPM } & \text { SPM } & \text { SPM } & \text { mitochondria } \\ \text { SPM } & \text { SPM } & \text { SPM } & 5.0 & 2.1 \text { mitochondria } \\ & 8.1 & \text { SPM } & \text { SPM } & \text { mitochondria } \\ 3.7 & 6.5 & 2.7 & 4.3 & \text { mitochondria } \\ 33.2 & 2.2 & 3.7 & 13.0 & 17.5 \text { mitochondria } \\ 4.1 & 7.0 & 7.4 & 6.2 & 1.8 \text { mitochondria } \\ 5.0 & \text { SPM } & 7.9 & 6.5 & 2.0 \text { mitochondria } \\ \text { SPM } & 5.8 & \text { SPM } & \text { SPM } & \text { mitochondria } \\ & \text { SPM } & \text { SPM } & \text { SPM } & \text { mitochondria } \\ \text { SPM } & 7.3 & \text { SPM } & \text { SPM } & \text { mitochondria } \\ 3.7 & 2.8 & 5.6 & 4.1 & 1.4 \text { mitochondria } \\ & 3.4 & \text { SPM } & & \text { mitochondria } \\ \text { SPM } & \text { SPM } & \text { SPM } & \text { SPM } & \text { mitochondria } \\ \text { SPM } & \text { SPM } & \text { SPM } & \text { SPM } & \text { mitochondria } \\ \text { SPM } & 7.2 & 8.8 & 8.0 & 1.2 \text { mitochondria } \\ \text { SPM } & \text { SPM } & 5.1 & \text { SPM } & \text { mitochondria } \\ & \text { SPM } & \text { SPM } & \text { SPM } & \text { mitochondria } \\ \text { SPM } & \text { SPM } & \text { SPM } & \text { SPM } & \text { mitochondria } \\ \text { SPM } & 8.2 & 9.3 & 8.8 & 0.8 \text { mitochondria } \\ 7.7 & 2.9 & 5.0 & 5.2 & 2.4 \text { mitochondria } \\ 4.6 & 6.3 & 8.4 & 6.4 & 1.9 \text { mitochondria } \\ 2.7 & 5.1 & 2.6 & 3.5 & 1.4 \text { mitochondria } \\ 4.5 & 8.4 & 6.7 & 6.5 & 2.0 \text { mitochondria } \\ \text { SPM } & 5.5 & 6.9 & 6.2 & 1.0 \text { mitochondria } \\ 2.1 & \text { SPM } & 3.4 & 2.8 & 0.9 \text { mitochondria } \\ \text { SPM } & \text { SPM } & 10.0 & \text { SPM } & \text { mitochondria } \\ \text { SPM } & \text { SPM } & \text { SPM } & \text { SPM } & \text { mitochondria } \\ \text { SPM } & 5.0 & 6.8 & 5.9 & 1.3 \text { mitochondria } \\ 8.0 & 7.5 & 5.1 & 6.9 & 1.5 \text { mitochondria } \\ 7.2 & \text { SPM } & \text { SPM } & \text { SPM } & \text { mitochondria } \\ & \text { SPM } & \text { SPM } & \text { SPM } & \text { mitochondria } \\ 4.9 & 7.3 & 7.1 & 6.5 & 1.3 \text { mitochondria } \\ \text { SPM } & 5.0 & \text { SPM } & \text { SPM } & \text { mitochondria } \\ \text { SPM } & \text { SPM } & \text { SPM } & \text { SPM } & \text { mitochondria } \\ \text { SPM } & \text { SPM } & \text { SPM } & \text { SPM } & \text { mitochondria } \\ 4.6 & 5.8 & 8.3 & 6.2 & 1.9 \text { mitochondria } \\ & \text { SPM } & \text { SPM } & \text { SPM } & \text { mitochondria } \\ & & & & \\ & & & & \end{array}$


GI number \# identified in protein name biological biological biological biological average standard protein localization/function replicate replicate replicate enrichment deviation

\begin{tabular}{|c|c|}
\hline gi|62945278 & 3 oxoglutarate (alpha-ketoglutarate) dehydrogenase (lipoamide) \\
\hline gi|157819765 & 2 oxoglutarate dehydrogenase-like \\
\hline gi|157819923 & 2 patatin-like phospholipase domain containing 8 \\
\hline gi|11968132 & 3 peroxiredoxin 3 \\
\hline gi|16758404 & 3 peroxiredoxin 5 precursor \\
\hline gi|70608189 & 2 phosphoglycerate mutase family member 5 \\
\hline gi|13937353 & 3 prohibitin \\
\hline gi|61556754 & 3 prohibitin 2 \\
\hline gi|122427836 & 2 protein phosphatase $2 C$, magnesium dependent, catalytic subunit \\
\hline gi|157823607 & 3 pyrroline-5-carboxylate synthetase (glutamate gamma-semialdehyde synthetase) \\
\hline gi|31543464 & 3 pyruvate carboxylase \\
\hline gi|124430510 & 2 pyruvate dehydrogenase (lipoamide) alpha 1 \\
\hline gi|56090293 & 3 pyruvate dehydrogenase (lipoamide) beta \\
\hline gi|113205496 & 3 pyruvate dehydrogenase complex, component $\mathrm{X}$ \\
\hline gi|32452540 & 2 ras homolog gene family, member $\mathrm{T} 2$ \\
\hline gi|148747459 & 3 RN protein \\
\hline gi|58865994 & 3 sideroflexin 1 \\
\hline gi $\mid 12621120$ & 3 sideroflexin 3 \\
\hline gi|23463279 & 3 sideroflexin 5 \\
\hline gi|34854800 & 3 solute carrier family 25 (mitochondrial carrier, Aralar), member 12 \\
\hline gi|62078785 & 3 solute carrier family 25 (mitochondrial carrier, glutamate), member 22 \\
\hline gi|109464795 & 2 solute carrier family 25, member 31 \\
\hline gi|20806141 & 3 solute carrier family 25 (mitochondrial carrier; phosphate carrier), member 3 \\
\hline gi|8394297 & 3 solute carrier family 25 , member 1 precursor \\
\hline gi|32189355 & 3 solute carrier family 25, member 4 \\
\hline gi|189491614 & 3 solute carrier family 25 , member 46 \\
\hline gi|32189350 & 3 solute carrier family 25 , member 5 \\
\hline gi|51948454 & 3 sorting and assembly machinery component 50 homolog \\
\hline gi|18426858 & 3 succinate dehydrogenase complex, subunit A, flavoprotein ( $F p$ ) \\
\hline gi|209915614 & 3 succinate dehydrogenase complex, subunit B, iron sulfur (Ip) \\
\hline gi|53850596 & 3 succinate dehydrogenase complex, subunit $\mathrm{C}$ \\
\hline gi|38454310 & 2 succinate dehydrogenase complex, subunit $D$, integral membrane protein \\
\hline gi|109504901 & 2 Succinate semialdehyde dehydrogenase \\
\hline gi|139948224 & 3 succinate-CoA ligase, GDP-forming, alpha subunit \\
\hline gi|158749584 & 3 succinate-Coenzyme A ligase, ADP-forming, beta subunit \\
\hline gi|8394331 & 3 superoxide dismutase 2 \\
\hline gi|62078811 & 2 threonyl-tRNA synthetase 2 , mitochondrial \\
\hline gi|47058998 & 2 TOM22 protein \\
\hline
\end{tabular}

$\# 1$

\#2

\begin{tabular}{rrrrr}
6.3 & 7.5 & 5.2 & 6.3 & 1.1 mitochondria \\
& SPM & 3.1 & & mitochondria \\
& SPM & 7.2 & SPM & mitochondria \\
SPM & 2.8 & SPM & 2.8 & mitochondria \\
SPM & 6.2 & 4.3 & 5.3 & 1.3 mitochondria \\
SPM & SPM & SPM & SPM & mitochondria \\
SPM & 7.7 & SPM & SPM & mitochondria \\
9.0 & 5.0 & 5.2 & 6.4 & 2.3 mitochondria \\
& SPM & SPM & SPM & mitochondria \\
SPM & SPM & SPM & SPM & mitochondria \\
3.3 & SPM & 5.3 & 4.3 & 1.5 mitochondria \\
& 6.5 & 5.2 & 5.8 & 0.9 mitochondria \\
9.3 & 6.5 & 5.7 & 7.2 & 1.9 mitochondria \\
SPM & 4.5 & SPM & SPM & mitochondria \\
& SPM & SPM & SPM & mitochondria \\
9.5 & 4.9 & 5.0 & 6.5 & 2.7 mitochondria \\
8.4 & 9.6 & 7.0 & 8.4 & 1.3 mitochondria \\
SPM & 8.2 & 2.9 & 5.6 & 3.8 mitochondria \\
SPM & SPM & 5.0 & SPM & mitochondria \\
SPM & 9.3 & 7.8 & 8.6 & 1.1 mitochondria \\
5.0 & 6.8 & 10.1 & 7.3 & 2.6 mitochondria \\
& SPM & SPM & SPM & mitochondria \\
6.5 & 7.6 & 10.2 & 8.1 & 1.9 mitochondria \\
SPM & 9.7 & 8.8 & 9.3 & 0.6 mitochondria \\
3.7 & 6.3 & 8.0 & 6.0 & 2.1 mitochondria \\
SPM & SPM & 3.4 & SPM & mitochondria \\
4.4 & 6.1 & 8.8 & 6.4 & 2.2 mitochondria \\
SPM & SPM & 5.4 & SPM & mitochondria \\
2.1 & 5.8 & 6.5 & 4.8 & 2.4 mitochondria \\
6.1 & 6.6 & 9.9 & 7.5 & 2.1 mitochondria \\
SPM & 5.0 & 7.4 & 6.2 & 1.7 mitochondria \\
& SPM & SPM & SPM & mitochondria \\
7.2 & SPM & 7.0 & SPM & mitochondria \\
SPM & 8.7 & 7.5 & 7.8 & 0.8 mitochondria \\
SPM & SPM & SPM & SPM & mitochondria \\
& 5.8 & 4.8 & 5.3 & 0.7 mitochondria \\
& SPM & SPM & SPM & mitochondria \\
& & SPM & SPM & mitochondria \\
\hline & & &
\end{tabular}


GI number \# identified in protein name biological

\begin{tabular}{|c|c|}
\hline gi|25742598 & 3 translocase of inner mitochondrial membrane 10 homolog \\
\hline gi|8394449 & 2 translocase of inner mitochondrial membrane 44 homolog \\
\hline gi|109461526 & 3 translocase of inner mitochondrial membrane 50 homolog isoform 2 \\
\hline gi|23097350 & 2 translocase of outer mitochondrial membrane 20 homolog \\
\hline gi|47058988 & 3 translocase of outer mitochondrial membrane 70 homolog $\mathrm{A}$ \\
\hline gi|157821331 & 2 transmembrane and coiled-coil domain family 3 \\
\hline gi|157820845 & 3 Tu translation elongation factor, mitochondrial \\
\hline gi|55741544 & 3 ubiquinol cytochrome $\mathrm{c}$ reductase core protein 2 \\
\hline gi|109458613 & 3 ubiquinol-cytochrome $\mathrm{c}$ reductase binding protein \\
\hline gi|109500943 & 3 ubiquinol-cytochrome c reductase complex $7.2 \mathrm{kDa}$ protein isoform a \\
\hline gi|51948476 & 3 ubiquinol-cytochrome c reductase core protein I \\
\hline gi|57164091 & 3 ubiquinol-cytochrome $\mathrm{c}$ reductase hinge protein \\
\hline gi|109481568 & 2 ubiquinol-cytochrome $\mathrm{c}$ reductase subunit \\
\hline gi|68341999 & 3 ubiquinol-cytochrome c reductase, complex III subunit VII \\
\hline gi| 57114330 & 3 ubiquinol-cytochrome $\mathrm{c}$ reductase, Rieske iron-sulfur polypeptide 1 \\
\hline gi|13786200 & 3 voltage-dependent anion channel 1 \\
\hline gi|13786202 & 3 voltage-dependent anion channel 2 \\
\hline gi||13786204 & 3 voltage-dependent anion channel 3 \\
\hline gi|56605990 & 3 leucine-rich PPR-motif containing \\
\hline gi|157822161 & 2 catechol-O-methyltransferase domain containing 1 \\
\hline gi|67846070 & 2 hydroxysteroid dehydrogenase like 1 \\
\hline gi|71043858 & 2 hydroxysteroid dehydrogenase like 2 \\
\hline gi|197927166 & 2 1-acylglycerol-3-phosphate O-acyltransferase 5 \\
\hline gi|157821573 & 3 ganglioside-induced differentiation-associated protein 1-like 1 \\
\hline gi|157821895 & 3 ganglioside-induced differentiation-associated-protein 1 \\
\hline gi| 109474876 & 2 hypothetical protein \\
\hline gi|77732522 & 2 outer membrane protein \\
\hline gi|158749559 & 3 bassoon protein \\
\hline gi|11559947 & 2 calcium/calmodulin-dependent serine protein kinase \\
\hline gi|25140983 & 3 ELKS/RAB6-interacting/CAST family member 2 \\
\hline gi|109497902 & 3 Liprin-alpha-4 \\
\hline gi|10048483 & 2 piccolo isoform 1 \\
\hline gi|157824053 & 2 liprin alpha 2 \\
\hline gi|16306470 & 3 regulating synaptic membrane exocytosis 1 \\
\hline gi|213972596 & 3 protein tyrosine phosphatase, receptor-type, $F$ interacting protein, binding protein 2 \\
\hline gi|9507073 & 3 neuroplastin \\
\hline gi| 46048609 & 3 beta-catenin \\
\hline gi| 157817081 & 2 catenin (cadherin associated protein), alpha 2 \\
\hline
\end{tabular}

biological biological biological average standard protein localization/function replicate replicate replicate enrichment deviation

$\begin{array}{rrr}\# 1 & \# 2 & \\ & & \\ \text { SPM } & 8.2 & \text { SPM } \\ & \text { SPM } & 9 . \\ \text { SPM } & \text { SPM } & \text { SPM } \\ & \text { SPM } & \text { SPM } \\ 2.6 & 9.1 & \text { SPM } \\ & \text { SPM } & \text { SPM } \\ 8.0 & 5.0 & 5 . \\ 7.7 & 6.8 & 5.9 \\ 4.9 & 4.5 & 5 . \\ \text { SPM } & \text { SPM } & \text { SPM } \\ 4.1 & 4.6 & 6.7 \\ \text { SPM } & 6.5 & \text { SPM } \\ & \text { SPM } & \text { SPM } \\ 4.1 & 4.8 & 8.1 \\ 3.3 & 10.3 & 4.5 \\ 6.3 & 3.2 & 6.0 \\ \text { SPM } & 2.7 & 6.1 \\ \text { SPM } & 4.2 & 11.3 \\ \text { SPM } & \text { SPM } & 8.2 \\ & \text { SPM } & \text { SPM } \\ & 5.8 & \text { SPM } \\ & \text { SPM } & \text { SPM } \\ & \text { SPM } & \text { SPM } \\ \text { SPM } & 3.9 & \text { SPM } \\ \text { SPM } & 3.1 & 3.4 \\ & \text { SPM } & \text { SPM } \\ & \text { SPM } & 4.2 \\ \text { SPM } & 8.0 & 6.8 \\ & \text { SPM } & \text { SPM } \\ 6.3 & \text { SPM } & \text { SPM } \\ \text { SPM } & \text { SPM } & 9.2 \\ & \text { SPM } & \text { SPM } \\ & \text { SPM } & 9.2 \\ \text { SPM } & 6.6 & 6.0 \\ 0.5 & 0.2 & 0.4 \\ \text { SPM } & 5.6 & 5.9 \\ 9.4 & 4.5 & \text { SPM } \\ & \text { SPM } & 4.6 \\ & & \\ & & \end{array}$

3

SPM mitochondria

$\begin{array}{rll}9.9 & \text { SPM } & \text { mitochondria }\end{array}$

SPM SPM mitochondria

SPM SPM

mitochondria

4.6 mitochondria

1.7 mitochondria

0.9 mitochondria

0.2 mitochondria

mitochondria

mitochondria

mitochondria

2.2 mitochondria

3.7 mitochondria

1.7 mitochondria

2.4 mitochondria

5.1 mitochondria

mitochondria

mitochondria

mitochondria

mitochondria

mitochondria

0.2 mitochondria

mitochondria

mitochondria
Active zone

Active zone

Active zone

Active zone

Active zone

Active zone

0.4 Active zone

0.4 Active zone

0.2 Adhesion and cell surface molecules

3.5 Adhesion and cell surface molecules
Adhesion and cell surface molecules 
GI number \#identified in protein name biological

\begin{tabular}{|c|c|}
\hline gi|157820047 & 2 catenin (cadherin associated protein), $d$ \\
\hline gi|109464562 & 3 Catenin delta-2 (Neurojungin) \\
\hline gi|114052921 & 2 cell adhesion molecule 3 \\
\hline gi|14091742 & 2 contactin associated protein 1 \\
\hline gi|157817598 & 3 inversin \\
\hline gi|157817019 & 2 plakophilin 4 \\
\hline gi|9506469 & $2 \mathrm{Cd} 47$ molecule \\
\hline gi|13928706 & 2 neural cell adhesion molecule 1 \\
\hline gi|61557326 & 3 receptor accessory protein 6 \\
\hline gi|30017437 & 3 glycoprotein m6a \\
\hline gi|20301986 & 2 glycoprotein m6b \\
\hline gi| 8393415 & 3 growth associated protein 43 \\
\hline gi|8850221 & 2 hippocalcin \\
\hline gi| 8393864 & 3 hippocalcin-like 1 \\
\hline gi|31543529 & 2 signal-regulatory protein alpha \\
\hline gi|6981654 & 3 Thy- 1 cell surface antigen \\
\hline gi|109478967 & 2 cysteine rich transmembrane BMP regul \\
\hline gi|109481923 & 2 centrosome protein cep 290 \\
\hline gi|109499926 & 2 spindle assembly associated Sfi1 homolo \\
\hline gi|13242237 & 2 heat shock protein 8 \\
\hline gi|28467005 & 2 heat shock protein 90, alpha (cytosolic), \\
\hline gi|70794764 & 2 DnaJ (Hsp40) homolog, subfamily A, mer \\
\hline gi|157822779 & 2 DnaJ (Hsp40) homolog, subfamily $C$, mer \\
\hline gi|6981324 & 3 prolyl 4-hydroxylase, beta polypeptide \\
\hline gi|84370227 & 2 DnaJ (Hsp40) homolog, subfamily A, mer \\
\hline gi|84781723 & 3 TNF receptor-associated protein 1 \\
\hline gi|72255527 & 3 stomatin (Epb7.2)-like 2 \\
\hline gi|6981696 & 2 utrophin \\
\hline gi|148491097 & 2 cytoplasmic dynein 1 heavy chain 1 \\
\hline gi|9506371 & 2 actin, alpha 1 , skeletal muscle \\
\hline gi|13592133 & 3 actin, beta \\
\hline gi|13591902 & 2 actinin, alpha 1 \\
\hline gi|57164143 & 2 ARP2 actin-related protein 2 homolog \\
\hline gi|109480041 & 3 formin 3 CG33556-PA \\
\hline gi|13540714 & 3 plectin 1 \\
\hline gi|11560133 & 3 tubulin, alpha $1 \mathrm{~A}$ \\
\hline gi|112984124 & 3 tubulin, alpha $1 B$ \\
\hline gi|55741524 & 2 tubulin, alpha $4 \mathrm{~A}$ \\
\hline
\end{tabular}

biological biological biological average standard protein localization/function replicate replicate replicate enrichment deviation

$\# 1$

$\# 2 \quad \# 3$

$\begin{array}{rrrr}2.3 & \text { SPM } & \text { SPM } & \text { Adhesion and cell surface molecules }\end{array}$

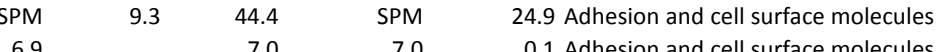

$\begin{array}{llll}6.9 & 7.0 & 7.0 & 0.1 \text { Adhesion and cell surface molecules }\end{array}$

SPM $\quad 7.9$

$\begin{array}{rr}7.9 & \text { SPM }\end{array}$

Adhesion and cell surface molecules Adhesion and cell surface molecules

1.1 Adhesion and cell surface molecules
2.0 Adhesion and cell surface molecules

0.7 Adhesion and cell surface molecules

0.7 Adhesion and cell surface molecules Adhesion and cell surface molecules Adhesion and cell surface molecules

1.3 Adhesion and cell surface molecules Adhesion and cell surface molecules 0.1 Adhesion and cell surface molecules 0.7 Adhesion and cell surface molecules

0.3 Adhesion and cell surface molecules Adhesion and cell surface molecules centrosome

centrosome

1.3 Chaperones
Chaperones

0.1 Chaperones

Chaperones

1.9 Chaperones

Chaperones

Chaperones

Cytoskeletal and associated proteins Cytoskeletal and associated proteins Cytoskeletal and associated proteins 0.8 Cytoskeletal and associated proteins 2.4 Cytoskeletal and associated proteins Cytoskeletal and associated proteins 1.4 Cytoskeletal and associated proteins 0.1 Cytoskeletal and associated proteins 1.6 Cytoskeletal and associated proteins 3.0 Cytoskeletal and associated proteins
2.6 Cytoskeletal and associated proteins 0.8 Cytoskeletal and associated proteins 
GI number \#identified in protein name biological

\section{replicates}

2 myosin Va

2 septin 3

3 septin 5

3 septin 8

3 flotillin 1

3 flotillin 2

3 reticulon 1

2 reticulon 4 biological biological biological average standard protein localization/function replicate replicate replicate enrichment deviation

\#1

\section{tubulin, beta 3}

3 tubulin, beta 5

3 Dynein heavy chain at $16 \mathrm{~F} \mathrm{CG7092-PA}$

3 Dynein heavy chain at 89D CG1842-PA

3 dynein heavy chain domain 3

3 dynein, cytoplasmic, light peptide

2 kinesin family member $1 \mathrm{~B}$

2 Kinesin-like protein KIF2

3 myosin, heavy chain 10 , non-muscle

3 myosin, heavy chain 9 , non-muscle

2 Beta-2-syntrophin (Syntrophin 3)

2 Oxygen-regulated protein 1 (Retinitis pigmentosa RP1 protein homolog)

2 SPHK1 interactor, AKAP domain containing

3 alpha-spectrin 2

2 ankyrin 2 isoform 1

2 septin 7 isoform a

3 septin 9 isoform 2

2 spectrin, beta, erythrocytic

2 spectrin, beta, non-erythrocytic 1

3 adaptor-related protein complex 2, alpha 1 subunit

3 adaptor-related protein complex 2 , alpha 2 subunit

3 adaptor-related protein complex 2 , beta 1 subunit

3 adaptor-related protein complex 2 , mu 1 subunit

3 adaptor-related protein complex 2, sigma 1 subunit

3 clathrin, heavy chain $(\mathrm{Hc})$

3 SH3-domain GRB2-like endophilin B2

3 protein tyrosine phosphatase, receptor type, $\mathrm{N}$ polypeptide 2

2 inositol-requiring 1 alpha

3 thioredoxin domain containing 13

$\begin{array}{rrr}2.9 & 1.9 & 0 . \\ 3.0 & 2.2 & 1.1 \\ 3.0 & 2.2 & 1.1 \\ \text { SPM } & \text { SPM } & \text { SPM } \\ 2.2 & 0.2 & 1 . \\ \text { SPM } & 9.5 & \text { SPM } \\ \text { SPM } & \text { SPM } & 9 . \\ & \text { SPM } & \text { SPM } \\ & \text { SPM } & 3 . \\ & 2.9 & 2.5 \\ \text { SPM } & 4.4 & \text { SPM } \\ \text { SPM } & \text { SPM } & \text { SPM } \\ & 0.8 & \text { SPM } \\ & \text { SPM } & 10 . \\ & 0.8 & 0.9 \\ \text { SPM } & 7.3 & 7.2 \\ & \text { SPM } & 4 . \\ \text { SPM } & \text { SPM } & \\ \text { SPM } & 3.8 & 5 . \\ 3.9 & & 3.3 \\ \text { SPM } & 3.6 & 5 . \\ 0.6 & 1.2 & 1.2 \\ & 1.0 & 5.5 \\ & \text { SPM } & 7.3 \\ \text { SPM } & \text { SPM } & \text { SPM } \\ \text { SPM } & \text { SPM } & \text { SPM } \\ 0.9 & 0.7 & 0 . \\ 0.8 & 0.8 & 0.7 \\ 0.9 & 0.8 & 0.5 \\ 0.5 & 0.6 & 0.6 \\ 0.7 & 0.6 & 0.6 \\ 7.6 & 4.6 & 5.2 \\ 2.6 & 1.1 & 0.7 \\ 1.8 & 2.7 & 1.7 \\ \text { SPM } & 7.3 & 5 . \\ & \text { SPM } & \text { SPM } \\ & \text { SPM } & \text { SPM } \\ \text { SPM } & \text { SPM } & 6.4 \\ & & \end{array}$

$0.9 \quad 1.9$

$1.9 \quad 1.0$ Cytoskeletal and associated proteins

0.9 Cytoskeletal and associated proteins

1.0 Cytoskeletal and associated proteins Cytoskeletal and associated proteins

1.0 Cytoskeletal and associated proteins Cytoskeletal and associated proteins Cytoskeletal and associated proteins Cytoskeletal and associated proteins Cytoskeletal and associated proteins

0.2 Cytoskeletal and associated proteins Cytoskeletal and associated proteins Cytoskeletal and associated proteins Cytoskeletal and associated proteins Cytoskeletal and associated proteins

0.1 Cytoskeletal and associated proteins

0.0 Cytoskeletal and associated proteins Cytoskeletal and associated proteins Cytoskeletal and associated proteins

1.3 Cytoskeletal and associated proteins 0.4 Cytoskeletal and associated proteins 1.3 Cytoskeletal and associated proteins 0.4 Cytoskeletal and associated proteins

0.2 Cytosketal and associated protens Cytoskeletal and associated proteins Endocytosis-related proteins Endocytosis-related proteins 0.1 Endocytosis-related protein 0.1 Endocytosis-related proteins 0.2 Endocytosis-related proteins 0.0 Endocytosis-related proteins 0.1 Endocytosis-related proteins 1.6 Endocytosis-related protein 1.0 Endocytosis-related proteins 0.6 Endocytosis-related proteins

1.2 ER/Golgi ER/Golgi
ER/Gi ER/Golgi 
GI number \#identified in protein name biological

\section{replicates}

3 calnexin

3 hemicentin 1

3 F58G4.1

2 CG13957-PA

ATPase, Ca++ transporting slow twitch 2 isoform a

2 ADP-ribosylation factor GTPase activating protein 1

2 complement component 4 binding protein, alpha

2 ST3 beta-galactoside alpha-2,3-sialyltransferase 3

2 apolipoprotein B precursor

2 brain creatine kinase

2 glucose phosphate isomerase

3 glyceraldehyde-3-phosphate dehydrogenase

2 leucine aminopeptidase 3

phosphofructokinase, muscle

3 phosphofructokinase, platelet

2 aldehyde dehydrogenase family 7 , member A1

2 glutamine synthetase 1

$23^{\sim}$-phosphoadenosine $5^{\sim}$-phosphosulfate synthase 2

3 fumarylacetoacetate hydrolase domain containing $2 \mathrm{~A}$

2 TRAF3-interacting JNK-activating modulator

3 BM88 antigen

2 OCIA domain containing 1

2 tumor suppressor candidate 5

3 coiled-coil domain containing 109A

2 thioredoxin-related transmembrane protein 2

3 transmembrane protease, serine 4

3 transmembrane protein 30A

2 WD repeat membrane protein PWDMP

3 transmembrane protease, serine 13

3 family with sequence similarity 162 , member A

2 CG7896-PA isoform 1

2 hypothetical protein

2 hypothetical protein

hypothetical protein

3 hypothetical protein LOC287559

2 hypothetical protein LOC296968 biological biological biological average standard protein localization/function replicate replicate replicate enrichment deviation

$\# 1$

\#2 \#3

$\begin{array}{rrrrc}\text { SPM } & 7.2 & 8.0 & 7.6 & 0.6 \text { ER/Golgi } \\ \text { SPM } & 4.1 & 5.2 & 4.6 & 0.8 \text { ER/Golgi } \\ & \text { SPM } & \text { SPM } & \text { SPM } & \text { ER/Golgi } \\ & \text { SPM } & \text { SPM } & \text { SPM } & \text { ER/Golgi } \\ & \text { SPM } & \text { SPM } & \text { SPM } & \text { Extracellular } \\ \text { SPM } & \text { SPM } & \text { SPM } & \text { SPM } & \text { Extracellular } \\ \text { SPM } & \text { SPM } & & \text { SPM } & \text { Extracellular } \\ & \text { SPM } & \text { SPM } & \text { SPM } & \text { Extracellular }\end{array}$

0.2 Metabolic enzymes

Metabolic enzymes

$\begin{array}{rrrrr}\text { SPM } & & \text { SPM } & \text { SPM } & \text { Metabolic enzymes } \\ 0.3 & 0.4 & 0.3 & 0.3 & 0.0 \text { Metabolic enzymes }\end{array}$

$\begin{array}{rrrrr}0.3 & 0.4 & 0.3 & 0.3 & 0.0 \text { Metabolic enzymes }\end{array}$

Metabolic enzymes

0.2 Metabolic enzymes

Metabolic enzymes

0.1 Metabolic enzymes

Metabolic enzymes

Metabolic enzymes

Novel

Nove

Novel

0.2 Novel

0.1 Nove

Novel

Novel

0.6 Nove

Novel

0.1 Novel

5.7 Novel

7 Novel
Novel
Novel

Novel

Novel

Nove

0.3 Novel 
GI number \# identified in protein name

biological

replicates biological biological biological average standard protein localization/function replicate replicate replicate enrichment deviation

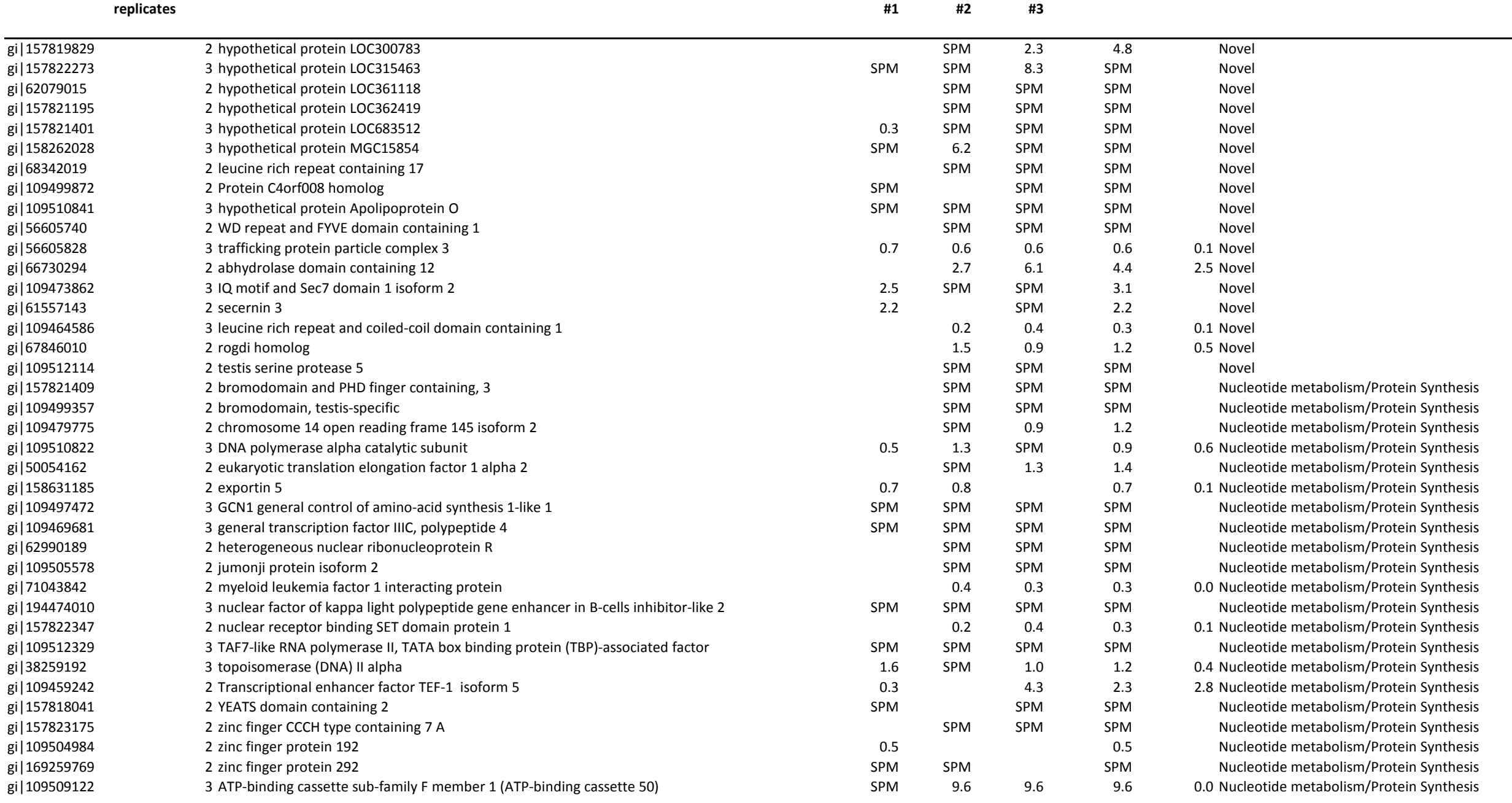


GI number \# identified in protein name biological

\begin{tabular}{|c|c|}
\hline gi|13592077 & 2 ribosomal protein $\mathrm{S} 27 \mathrm{a}$ \\
\hline gi|34328536 & 2 single-stranded DNA binding protein 1 \\
\hline gi| 55926145 & 3 non-metastatic cells 2, protein (NM23B) expressed in \\
\hline gi|9665227 & 2 post-synaptic density protein 95 \\
\hline gi|164663821 & 3 synaptic Ras GTPase activating protein 1 homolog isoform 1 \\
\hline gi|14091744 & 2 kalirin, RhoGEF kinase \\
\hline gi|109483754 & 2 growth and transformation-dependent protein \\
\hline gi| 109483500 & 2 Dmx-like 2 \\
\hline gi|109503754 & 2 WD repeat and FYVE domain containing 3 \\
\hline gi|109481805 & 3 CG1814-PA, isoform A \\
\hline gi|109479730 & 2 CG33714-PB, isoform B \\
\hline gi|109470173 & 3 FU44048 protein \\
\hline gi|109497812 & 3 hypothetical protein \\
\hline gi|109514756 & 2 hypothetical protein LOC363337 \\
\hline gi|8394267 & 2 sonic hedgehog \\
\hline gi|9507061 & 3 proprotein convertase subtilisin/kexin type 1 inhibitor \\
\hline gi|109458044 & 2 PYRIN-containing APAF1-like protein 7 isoform 2 \\
\hline gi|157818451 & 2 ADP-ribosylation factor-like $8 \mathrm{~A}$ \\
\hline gi|12408298 & 2 dipeptidylpeptidase 6 \\
\hline gi|109475021 & 2 CDK5 regulatory subunit associated protein 2 \\
\hline gi|13592021 & 2 phosphodiesterase $2 A$, cGMP-stimulated isoform 2 \\
\hline gi|6978593 & 3 calcium/calmodulin-dependent protein kinase II alpha \\
\hline gi|108796657 & 3 calcium/calmodulin-dependent protein kinase II beta isoform2 \\
\hline gi|6978595 & 2 calcium/calmodulin-dependent protein kinase II delta \\
\hline gi|19424316 & 3 calcium/calmodulin-dependent protein kinase II gamma \\
\hline gi|155369271 & 2 cAMP-dependent protein kinase catalytic subunit alpha \\
\hline gi|109464256 & 3 cardiomyopathy associated 5 \\
\hline gi|6981400 & 2 protein kinase C, gamma \\
\hline gi|157822659 & 2 RIO kinase 3 \\
\hline gi|132626321 & 2 serine/threonine kinase 2 \\
\hline gi|6981712 & 2 14-3-3 theta polypeptide \\
\hline gi|62990183 & 2 14-3-3, zeta polypeptide \\
\hline gi|25742825 & 2 phosphatidylinositol 4-kinase a \\
\hline gi|9506737 & 2 GNAS complex locus gnas1-a \\
\hline gi|109487963 & 3 dedicator of cyto-kinesis 2 \\
\hline gi|42476092 & $2 \mathrm{G}$ protein pathway suppressor 1 \\
\hline gi|8394152 & 3 GTP-binding protein alpha o \\
\hline gi|6980962 & 2 guanine nucleotide binding protein (G protein), alpha inhibiting \\
\hline
\end{tabular}

biological biological biological average standard protein localization/function replicate replicate replicate enrichment deviation

$\begin{array}{rrr}3.8 & 4.1 & 0.4 \text { Nucleotide metabolism/Protein Synthesis } \\ \text { SPM } & \text { SPM } & \text { Nucleotide metabolism/Protein Synthesis } \\ 8.5 & 8.7 & 0.4 \text { Nucle }\end{array}$

$\begin{array}{rrrrr} & \text { SPM } & \text { SPM } & \text { SPM } & \text { Nucleotide metabolism/Protein Synthesis } \\ \text { SPM } & 9.0 & 8.5 & 8.7 & 0.4 \text { Nucleotide metabolism/Protein Synthesis }\end{array}$

Postsynaptic

1.9 Postsynaptic

Postsynaptic

record removed

record removed

record removed

0.6 record removed record removed

record removed

0.2 record removed

record removed

Signalling molecules
Signalling molecules

Signalling molecules

Signalling molecules

0.2 Signalling molecules

Signalling molecules

Signalling molecules

1.6 Signalling molecules

1.0 Signalling molecules

2.3 Signalling molecules

1.7 Signalling molecules Signalling molecules

0.6 Signalling molecules

0.1 Signalling molecule Signalling molecules Signalling molecules Signalling molecules Signalling molecules Signalling molecules

0.2 Signalling molecules

0.0 Signalling molecules

1.8 Signalling molecules 
GI number \# identified in protein name biological replicates biological biological biological average standard protein localization/function replicate replicate replicate enrichment deviation

\begin{tabular}{|c|c|c|c|c|c|c|}
\hline gi|13591957 & 2 guanine nucleotide binding protein, alpha q polypeptide & & SPM & 3.8 & 6.2 & Signalling molecules \\
\hline gi|109507443 & 2 Guanine nucleotide-binding protein $\mathrm{G}(\mathrm{olf})$, alpha subunit & & 8.9 & SPM & SPM & Signalling molecules \\
\hline gi|29789261 & 2 guanine nucleotide-binding protein, beta 2 & & 6.3 & 8.7 & 7.5 & 1.7 Signalling molecules \\
\hline gi|148747524 & 3 guanine nucleotide-binding protein, beta- 1 subunit & 9.0 & 6.3 & 8.7 & 8.0 & 1.5 Signalling molecules \\
\hline gi|19173774 & 2 RAP2B, member of RAS oncogene family & & SPM & 5.2 & 4.7 & Signalling molecules \\
\hline gi|157820415 & 3 RAS protein activator like 1 (GAP1 like) & 10.0 & 8.4 & 8.7 & 9.0 & 0.8 Signalling molecules \\
\hline gi|13592039 & 2 ras related $\mathrm{v}$-ral simian leukemia viral oncogene homolog $A$ & & SPM & SPM & SPM & Signalling molecules \\
\hline gi|157821177 & 3 triple functional domain (PTPRF interacting) & SPM & SPM & SPM & SPM & Signalling molecules \\
\hline gi|62079163 & 3 ATG 9 autophagy related 9 homolog A & 1.4 & 0.6 & 1.5 & 1.2 & 0.5 SNARE proteins and trafficking molecules \\
\hline gi|13027430 & 2 WD repeat domain 7 & & SPM & 1.8 & 2.5 & SNARE proteins and trafficking molecules \\
\hline gi|6981602 & 3 syntaxin binding protein 1 & 2.5 & 2.1 & 2.2 & 2.3 & 0.2 SNARE proteins and trafficking molecules \\
\hline gi|9507127 & 3 SNAP25-interacting protein & 3.9 & 6.2 & 7.7 & 5.9 & 1.9 SNARE proteins and trafficking molecules \\
\hline gi|13489067 & $3 \mathrm{~N}$-ethylmaleimide-sensitive factor & 5.1 & 2.4 & 2.2 & 3.3 & 1.6 SNARE proteins and trafficking molecules \\
\hline gi|219275534 & 2 vacuolar protein sorting 13 homolog $A$ & & 2.7 & 2.9 & 2.8 & 0.1 SNARE proteins and trafficking molecules \\
\hline gi|25742604 & 2 vacuolar protein sorting 45 homolog & & SPM & SPM & SPM & SNARE proteins and trafficking molecules \\
\hline gi|12408324 & 2 complexin 1 & 7.0 & 3.7 & & 5.3 & 2.3 SNARE proteins and trafficking molecules \\
\hline gi|109471437 & 2 Syntaxin-16 (Syn16) isoform 1 & & SPM & SPM & SPM & SNARE proteins and trafficking molecules \\
\hline gi|13591882 & 3 synaptosomal-associated protein 25 & 4.3 & 4.2 & 4.1 & 4.2 & 0.1 SNARE proteins and trafficking molecules \\
\hline gi|33667087 & 3 syntaxin $1 \mathrm{~A}$ (brain) & 3.0 & 2.9 & 3.1 & 3.0 & 0.1 SNARE proteins and trafficking molecules \\
\hline gi|6981600 & 3 syntaxin $1 \mathrm{~B} 2$ & 3.5 & 3.4 & 3.4 & 3.4 & 0.1 SNARE proteins and trafficking molecules \\
\hline gi|76443677 & 3 vesicle-associated membrane protein 1 & 0.9 & 0.8 & 0.9 & 0.9 & 0.1 SNARE proteins and trafficking molecules \\
\hline gi|6981614 & 3 vesicle-associated membrane protein 2 & 0.7 & 0.7 & 0.8 & 0.7 & 0.0 SNARE proteins and trafficking molecules \\
\hline gi|45433570 & 3 RAB1, member RAS oncogene family & 2.1 & 2.1 & 2.1 & 2.1 & 0.0 Synaptic vesicle \\
\hline gi|61889071 & 3 RAB10, member RAS oncogene family & 1.8 & 1.6 & 1.8 & 1.7 & 0.1 Synaptic vesicle \\
\hline gi|16758368 & 3 RAB14, member RAS oncogene family & 2.8 & 1.9 & 1.9 & 2.2 & 0.5 Synaptic vesicle \\
\hline gi|16758202 & 2 RAB27B, member RAS oncogene family & 2.6 & & SPM & 2.5 & Synaptic vesicle \\
\hline gi|13929006 & 3 RAB2A, member RAS oncogene family & 1.9 & 1.8 & 2.0 & 1.9 & 0.1 Synaptic vesicle \\
\hline gi|61556789 & 3 RAB35, member RAS oncogene family & 2.2 & 2.0 & 1.9 & 2.1 & 0.1 Synaptic vesicle \\
\hline gi|157822741 & 3 RAB39, member RAS oncogene family & 2.8 & 1.9 & 1.9 & 2.2 & 0.5 Synaptic vesicle \\
\hline gi|13592037 & 2 RAB3B, member RAS oncogene family & & 1.8 & 1.5 & 1.7 & 0.2 Synaptic vesicle \\
\hline gi|61098195 & 3 RAB3A, member RAS oncogene family & 2.3 & 1.9 & 1.9 & 2.0 & 0.2 Synaptic vesicle \\
\hline gi|158749626 & 3 secretory carrier membrane protein 1 & 3.2 & 1.2 & 1.0 & 1.8 & 1.2 Synaptic vesicle \\
\hline gi|13929020 & 3 secretory carrier membrane protein 5 & 0.6 & 0.6 & 0.6 & 0.6 & 0.0 Synaptic vesicle \\
\hline gi|109465077 & 2 Secretory carrier-associated membrane protein 3 & 1.0 & 1.1 & & 1.1 & 0.1 Synaptic vesicle \\
\hline gi|109499663 & 3 ATPase, aminophospholipid transporter (APLT), class I, type 8A, member 1 & 1.6 & 1.3 & 1.4 & 1.4 & 0.1 Synaptic vesicle \\
\hline gi|16758754 & 3 ATPase, $\mathrm{H}$ transporting, lysosomal V1 subunit $\mathrm{F}$ & 1.1 & 0.9 & 1.0 & 1.0 & 0.1 Synaptic vesicle \\
\hline gi|18677757 & 3 ATPase, $\mathrm{H}+$ transporting, lysosomal 16kDa, V0 subunit c & 0.6 & 0.7 & 0.8 & 0.7 & 0.1 Synaptic vesicle \\
\hline gi|58865424 & 3 ATPase, $\mathrm{H}+$ transporting, lysosomal 38kDa, V0 subunit d1 & 1.0 & 0.9 & 0.9 & 0.9 & 0.0 Synaptic vesicle \\
\hline
\end{tabular}


GI number \# identified in protein name biological

2 synapsin III

3 synaptophysin biological biological biological average standard protein localization/function replicate replicate replicate enrichment deviation

3 ATPase, $\mathrm{H}+$ transporting, Iysosomal accessory protein 1

ATPase, $\mathrm{H}+$ transporting, lysosomal V1 subunit C1

3 ATPase, $\mathrm{H}+$ transporting, lysosomal V1 subunit $\mathrm{D}$

3 ATPase, $\mathrm{H}+$ transporting, lysosomal V1 subunit G2

3 ATPase, $\mathrm{H}+$ transporting, lysosomal $\mathrm{V} 1$ subunit $\mathrm{H}$

3 ATPase, $\mathrm{H}+$ transporting, $V 1$ subunit $A$, isoform 1 isoform 1

3 cysteine string protein

3 MAL2 proteolipid protein

3 mossy-fiber terminal-associated vertebrate-specific presynaptic protein

3 solute carrier family 17 , member 6

3 solute carrier family 17 , member 7

3 solute carrier family 6 (neurotransmitter transporter), member 17

3 synapsin I isoform a

3 synapsin II isoform

3 synaptic vesicle glycoprotein $2 a$

3 synaptic vesicle glycoprotein $2 b$

3 synaptogyrin 1

3 synaptogyrin 3

2 synaptoporin

3 synaptotagmin 1

3 synaptotagmin II

2 synaptotagmin $V$

3 vacuolar $\mathrm{H}+$ ATPase E1

3 vesicular inhibitory amino acid transporter

3 zinc transporter $\mathrm{ZnT}-3$

3 inositol 1,4,5-triphosphate receptor, type 1

2 solute carrier family 1 (glial high affinity glutamate transporter), member 3

2 chloride channel 6

2 glial high affinity glutamate transporter isoform b

2 toll-like receptor 8

2 calcium channel, voltage-dependent, alpha2/delta subunit 1 isoform 1

2 calcium channel, voltage-dependent, beta 3 subunit

2 hyperpolarization-activated cyclic nucleotide-gated potassium channel 1 $3 \mathrm{Na}+/ \mathrm{K}+$-ATPase alpha 1 subunit

$\begin{array}{rrrr}0.9 & 0.8 & 0.9 & 0.9 \\ 0.9 & 0.9 & 0.9 & 0.9 \\ 1.1 & 0.9 & 0.9 & 1.0 \\ 1.1 & 1.1 & 1.0 & 1.1 \\ 0.8 & 0.9 & 0.9 & 0.9 \\ 0.9 & 0.9 & 0.9 & 0.9 \\ 0.9 & 0.9 & 0.9 & 0.9 \\ 1.2 & 1.2 & 0.9 & 1.1 \\ 0.9 & 0.8 & 0.8 & 0.8 \\ 0.9 & 0.5 & 1.0 & 0.8 \\ 1.0 & 0.8 & 0.8 & 0.9 \\ 1.0 & 0.8 & 0.8 & 0.9 \\ 1.7 & 1.4 & 1.4 & 1.5 \\ 2.9 & 1.2 & 1.6 & 1.9 \\ 1.7 & 1.4 & & 1.6 \\ & 1.8 & 1.9 & 1.8 \\ 0.8 & 0.8 & 0.8 & 0.8 \\ 1.0 & 0.8 & 0.9 & 0.9 \\ 0.5 & 0.6 & 0.6 & 0.6 \\ 0.5 & 0.5 & 0.4 & 0.5 \\ 0.6 & 0.7 & 0.7 & 0.7 \\ 0.9 & & 0.7 & 0.8 \\ 1.2 & 1.1 & 1.1 & 1.1 \\ 1.6 & 1.2 & 1.5 & 1.4 \\ & 1.2 & 1.5 & 1.3 \\ 0.9 & 1.1 & 0.9 & 1.0 \\ 0.9 & 0.9 & 0.9 & 0.9 \\ 0.7 & 0.8 & 0.9 & 0.8 \\ 0.8 & 0.8 & 0.8 & 0.8 \\ 6.2 & \text { SPM } & 7.6 & 6.9 \\ & 10.2 & \text { SPM } & \text { SPM } \\ & \text { SPM } & \text { SPM } & \text { SPM } \\ & 7.5 & 10.2 & 8.9 \\ & \text { SPM } & \text { SPM } & \text { SPM } \\ \text { SPM } & & 8.9 & \text { SPM } \\ & \text { SPM } & \text { SPM } & \text { SPM } \\ \text { SPM } & & \text { SPM } & \text { SPM } \\ 7.8 & 7.1 & 8.6 & 7.8 \\ & & & \end{array}$

\subsection{Synaptic vesicle}

0.0 Synaptic vesicle

0.2 Synaptic vesicle

0.0 Synaptic vesicle

0.0 Synaptic vesicle

0.0 Synaptic vesicle

0.1 Synaptic vesicle

0.0 Synaptic vesicle

0.3 Synaptic vesicle

0.1 Synaptic vesicle

0.1 Synaptic vesicle

0.2 synaptic vesicle

0.9 Synaptic vesicle

0.2 Synaptic vesicle

0.0 Synaptic vesicle

0.0 Synaptic vesicle

0.1 Synaptic vesicle

0.0 Synaptic vesicle

0.0 Synaptic vesicle

0.0 Synaptic vesicle

0.2 Synaptic vesicle

0.1 Synaptic vesicle

0.2 Synaptic vesicle

0.2 Synaptic vesicle

0.1 Synaptic vesicle

0.0 Synaptic vesicle

0.1 Synaptic vesicle

0.0 Synaptic vesicle

1.0 Transporter/Channel Proteins/Receptors Transporter/Channel Proteins/Receptors

Trans

Transpor/Chons Transoter/Chann Proin/Receptors Transpor/Chan Transporter/Channel Proteins/Receptors Transporter/Channel Proteins/Receptors
0.7 Transporter/Channel Proteins/Receptors 
GI number \# identified in protein name

biological

gi|6978547

gi $\mid 148747253$

gi|16758008

gi|6978557

gi|13929184

gi|19705463

gi| 148747140

gi|62644838

gi| 17530967

gi| 6981558

gi|13242269

gi|47576439
$3 \mathrm{Na}+/ \mathrm{K}+-\mathrm{ATPase}$ alpha 3 subunit

$\mathrm{Na} / \mathrm{K}+$-ATPase beta 1 subunit

3 plasma membrane calcium ATPase

2 plasma membrane

2 potassium large conductance calcium-activated channel, subfamily M, alpha member 1

3 solute carrier family 12 (potassium-chloride transporter), member 5

2 solute carrier family 2 (facilitated glucose transporter), member 3

3 solute carrier family 27 (fatty acid transporter), member 4

3 solute carrier family 8 (sodium/calcium exchanger), member 2

2 solute carrier family 9 (sodium/hydrogen exchanger), member 1

2 GABA transporter protein

2 olfactory receptor OIr1589 biological biological biological average standard protein localization/function

replicate replicate replicate enrichment deviation

\#1 \#2 \#3

8.0

6.8

7.3

0.6 Transporter/Channel Proteins/Receptors

1.2 Transporter/Channel Proteins/Receptors

2.8 Transporter/Channel Proteins/Receptors .9 Transporter/Channel Proteins/Recepto Transporter/Channel Proteins/Receptors 1.2 Transporter/Channel Proteins/Receptors

0.3 Transporter/Channel Proteins/Receptors Transporter/Channel Proteins/Receptors

1.1 Transporter/Channel Proteins/Receptors Transporter/Channel Proteins/Receptors Transporter/Channel Proteins/Receptors Transporter/Channel Proteins/Receptors 
gene name protein name

\begin{tabular}{|c|c|}
\hline Sept3 & septin 3 \\
\hline Sept5 & septin 5 \\
\hline Sirpa & signal regulatory protein alpha \\
\hline Slc17a7 & VGlut1 \\
\hline Slc1a2 & EAAT1 \\
\hline Slc1a3 & EAAT2 \\
\hline Slc30a3 & zinc transporter ZnT-3 \\
\hline Slc6a1 & GABA transporter protein \\
\hline Slc6a17 & neurotransmitter transporter NTT4 \\
\hline Snap25 & SNAP25 \\
\hline Sptan1 & spectrin, alpha \\
\hline Stx1b & syntaxin 1B \\
\hline Stxbp1 & Munc18-1 \\
\hline Syn1 & synapsin 1 \\
\hline Syn2 & synapsin 2 \\
\hline Syp & synaptophysin \\
\hline Syt1 & synaptotagmin 1 \\
\hline Thy1 & Thy- 1 cell surface antugen \\
\hline Tuba1a & tubulin, alpha $1 \mathrm{~A}$ \\
\hline Tuba1b & tubulin, alpha 1B \\
\hline Tubb3 & tubulin, beta 3 \\
\hline Tubb5 & tubulin, beta 5 \\
\hline Vamp1 & synaptobrevin 1 \\
\hline Vamp2 & synaptobrevin 2 \\
\hline Ywhaq & $14-3-3$ theta \\
\hline Ywhaz & 14-3-3 zeta \\
\hline Acta1 & actin, alpha 1 \\
\hline Actb & actin, beta \\
\hline Ap2b1 & AP2 complex, beta 1 \\
\hline Atp1a1 & $\mathrm{Na} / \mathrm{K}$ ATPase alpha 1 \\
\hline Atp1a3 & $\mathrm{Na} / \mathrm{K}$ ATPase alpha 3 \\
\hline Atp1b1 & $\mathrm{Na} / \mathrm{K}$ ATPase beta 1 \\
\hline Atp2b1 & PMCA 1 \\
\hline Atp6v0a1 & Iysosomal ATPase V0 a1 \\
\hline Atp6v0d1 & lysosomal ATPase V0 d1 \\
\hline Atp6v1b2 & lysosomal ATPase V1 b2 \\
\hline Atp6v1c1 & lysosomal ATPase V1 c1 \\
\hline Atp6v1d & lysosomal ATPase V1 d \\
\hline Atp6v1e1 & lysosomal ATPase V1 e1 \\
\hline Cacna2d1 & calcium channel a2/d subunit 1 \\
\hline Camk2a & CAMKII alpha \\
\hline Cd47 & CD47 molecule \\
\hline Cltc & clathrin heavy chain \\
\hline Cntnap1 & Neurexin 4 \\
\hline Dnajc5 & cysteine string protein \\
\hline Gnal & guanine nucleotide-binding protein $\mathrm{G}$ (olf) alpha \\
\hline Gnao1 & GTP-binding protein alpha o \\
\hline Gnaq & guanine nucleotide-binding protein alpha q \\
\hline Gnb1 & guanine nucleotide-binding protein beta 1 \\
\hline Gnb2 & guanine nucleotide-binding protein beta 2 \\
\hline Gpm6a & glycoprotein m6a \\
\hline Hsp90aa1 & heat shock protein 90 \\
\hline Hspa8 & heat shock protein 8 \\
\hline Ncam1 & neuronal cell adhesion molecule 1 \\
\hline Nptn & neuroplastin \\
\hline Nsf & $\mathrm{N}$-ethylmaleimide-sensitive factor \\
\hline Plec1 & plectin 1 \\
\hline Prkcg & protein kinase $C$, gamma \\
\hline Rab14 & Rab 15 \\
\hline Rab2a & $\operatorname{Rab} 2 \mathrm{a}$ \\
\hline Rab35 & Rab 35 \\
\hline Rab3a & Rab 3a \\
\hline
\end{tabular}




\begin{tabular}{|c|c|}
\hline gene name & protein name \\
\hline Ap2b1 & adaptor-related protein complex 2 , beta 1 subunit \\
\hline Ap2a1 & adaptor-related protein complex 2 , alpha 1 subunit \\
\hline Ap2a2 & adaptor-related protein complex 2 , alpha 2 subunit \\
\hline Ap2m1 & adaptor-related protein complex 2 , mu 1 subunit \\
\hline Atp6v0a1 & lysosomal ATPase V0a1 \\
\hline Atp6v1e1 & lysosomal ATPase V1 e1 \\
\hline Atp6v1a1 & lysosomal ATPase V1 a1 \\
\hline Atp6v1b2 & lysosomal ATPase V1 b2 \\
\hline Atp6v1c1 & lysosomal ATPase V1 c1 \\
\hline Atp6v0d1 & lysosomal ATPase V0 d1 \\
\hline Cltc & clathrin heavy chain \\
\hline Dync1h1 & cytoplasmic dynein 1 heavy chain 1 \\
\hline Nsf & $\mathrm{N}$-ethylmaleimide-sensitive factor \\
\hline Rab3a & Rab3a \\
\hline Rims1 & $\operatorname{Rim} 1$ \\
\hline Sh3gl2 & endophilin B2 \\
\hline Snap25 & SNAP25 \\
\hline Stx1a & syntaxin $1 \mathrm{~A}$ \\
\hline Stxbp1 & Munc18-1 \\
\hline Syn1 & synapsin 1 \\
\hline Syn2 & synapsin 2 \\
\hline Syt1 & synaptotagmin 1 \\
\hline Syt2 & synaptotagmin 2 \\
\hline Vamp2 & synaptobrevin 2 \\
\hline Camk2a & CAMKII alpha \\
\hline Gnao1 & GTP-binding protein alpha o \\
\hline Gnb1 & guanine nucleotide-binding protein beta 1 \\
\hline Gnb2 & guanine nucleotide-binding protein beta 2 \\
\hline Gnai1 & guanine nucleotide-binding protein alpha inhibiting 1 \\
\hline Ywhaz & 14-3-3 zeta \\
\hline Atp1a3 & $\mathrm{Na} / \mathrm{K}$ ATPase alpha 3 \\
\hline Ank2 & ankyrin 2 , neuronal \\
\hline Ncam1 & neuronal cell adhesion molecule 1 \\
\hline Acta1 & actin, alpha 1 \\
\hline Actb & actin, beta \\
\hline Actn1 & actinin, alpha 1 \\
\hline Sept3 & septin 3 \\
\hline Sept5 & septin 5 \\
\hline Sept7 & septin 7 \\
\hline Sptan1 & spectrin alpha chain, brain \\
\hline Sptbn1 & spectrin beta chain, brain 1 \\
\hline Tubb4 & tubulin, beta 4 \\
\hline Tubb3 & tubulin, beta 3 \\
\hline Tuba1a & tubulin, alpha $1 \mathrm{~A}$ \\
\hline Hsp90aa1 & heat shock protein 90 \\
\hline Hspa8 & heat shock protein 8 \\
\hline Thy1 & Thy- 1 cell surface antigen \\
\hline
\end{tabular}

